\title{
DISCLAIMER
}

This report was prepured as an vocotint of work aponuored by an arency of the United Statea Cowernmeat. Nedther tho Unitod Staten Gowarnmont nor any agency thoreof, nor uny of thoir

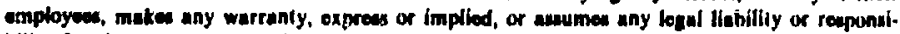
bltiky for the accuracy. complotenow, or usefulnow of any informution, apparalus, product, or procew dieclomed, or roprewenls that lis uso would not infringe privatoly owned rights. Refer-

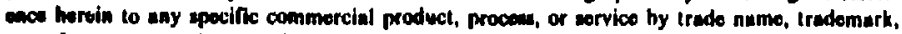
manufucturer, of otherwive does not nocmerily conattuto or imply its esidorwemont, rocom. meadation, of fuvoring by the United Stuted Government or any urency thereof. The vlown

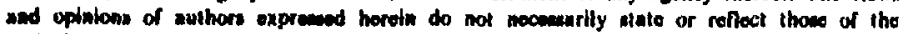
Uaited Stale Government or any ugency thereor.

UCRL -53887

DE89 002928

\section{Space and Time Resolved Spectroscopy of Laser- Produced Plasmas: A Study of Density-Sensitive X-ray Transitions in Helium-Like and Neon-Like Ions.}

\author{
Bruce Kai Fong Young \\ (Ph.D. Thesis)
}

Manuscript date: September 1988

\section{LAWRENCE LIVERMORE NATIONAL LABORATORY University of California - Livermore, California - 94550}

Avaflable from; National Technical Information Service - U.5. Departmunt of Commerre 5285 l'ort Royal Road - Springfield, VA 22161 - AO6 - (Microficho A(1)

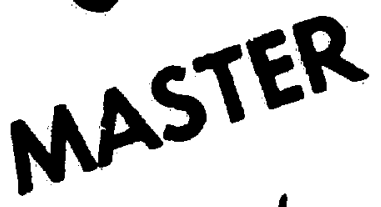


Space and Time Resolved Spectroscopy of Laser-Produced Plasmas:

A Study of Density-Sensitive X-ray Transitions in Helium-Like and Neon-Like Ions.

By

Bruce Kai Fong Young

\section{DISSERTATION}

Submitted in partial satisfaction of the requirements for the degree of DOCTOR OF PHILOSOPHY in

Engineering-Applied Science in the GRADUATE DIVISION of the

\section{UNIVERSITY OF CALIFORNIA}

DAVIS

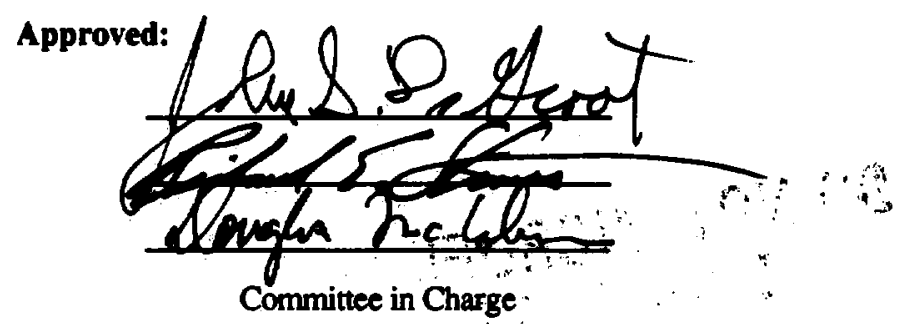

Deposited in the University Litrary 


\title{
Bruce Kai Fong Young
}

September 1988

Engineering-Applied Science

Space and Time Resolved Spectroscopy of Laser-Produced Plașmas:

A Study of Density-Sensitive X-ray Transitions in Helium-Like and Neon-Like Ions

\begin{abstract}
Abatract
The determination of level populations and detailed population mechanisms in dense plasmas has become an increasingly important problem in atomic physics. In the present work, the density variation of line intensities and level populations in aluminum K-shell and molybdenum and silver L-shell emission spectra have been measured from high-powered, laser-produced plasmas. For each case, the density dependence of the observed line emission is due to the effect of high frequency electron-ion collisions on metastable levels. The density dependent line intensities vary greatly in laser-produced plasmas and can be used to extract detailed information concerning the population kinetics and level populations of the ions.

The laser-plasmas had to be fully characterized in order to clearly compare the observed density dependence with atomic theory predictions. This has been achieved through the combined use of new diagnostic instruments and microdot targets which provided simultaneously space, time, and spectrally resolved data. The plasma temperatures were determined from the slope of the hydrogen-like recombination continuum. The time resolved electron density profiles were measured using multiple frame holographic interferometry. Thus, the density dependence of K-shell and L-shell spectral lines could be clearly examined,
\end{abstract}


independent of assumptions concerning the dynamics of the plasma.

In aluminum, the electron density dependence of various helium-like line intensity ratios were measured. Standard collisional radiative equilibrium models fail to account for the observed density dependence measured for the "He $/ \mathrm{IC}$ " ratio. Instead, a quasi-steady state atomic model based on a purely recombining plasma is shown to accurately predict the measured density dependence. This same recombining plasma calculation successfully models the density dependence of the

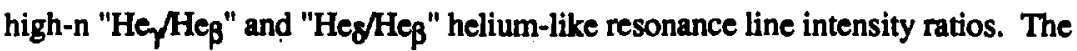
density dependence of metastable levels of neon-like ions is also measured. This is done by studying weak electric quadrupole transitions in neon-like molybdenum and silver ions. Detailed neon-like theoretical atomic model calculations are shown to correctly predict the observed density sensitivity of the electric quadrupole to dipole-allowed resonance line " $x / 3 \mathrm{~A}$ " ratio. The measured density dependence of the " $3 \mathrm{~A} / 3 \mathrm{G}$ " resonance line ratio does not agree with theory. Possible reasons for this discrepancy and suggestions for further experiments are reviewed. 


\section{Acknowledgments}

I wish to express my deep gratitude and appreciation to my many friends and colleagues who have shared their time, ideas, and enthusiasm with me throughout these past years. Foremost, I would like to posthumously thank Prof. Abe Goldberg. This dissertation would not exist without his constant encouragement and support. I wish to thank Prof. John DeGroot for kindly taking over as my thesis advisor and supporting me through the final stages of my research. I am especially grateful to Rick Stewart for his direction and guidance. He initially suggested the problem, and has played a crucial role in the planning, execution, and analysis of this work.

I wish to thank the members of the High Temperature Physics Division (V-Division) at LLNL for their technical and moral support. In particular, it has been a pleasure working with a very creative and enthusiastic group of experimenters including Dick Fortner, Rick Stewart, Dwight Price, Dan Dietrich, Ross Marrs, Pat Egan, Mort Levine, Chuck Hailey, Tom Phillips, Roger Morales, and Jim Bailey. I would also like to thank Tom, Leslie Burr, John Kennedy, and Rudy Bauer who assisted in the tedious task of data reduction and preliminary analysis of the data. I am very grateful to Rosemary Walling, Al Osterheld, Bill Goldstein, Charlie Cerjan, Mordy Rosen, Jim Scofield, Jeff Nash, Mau Chen, Andy Hazi, and Kennedy Reed for allowing me to use their theoretical calculations and for greatly expanding my knowledge of atomic physics and hydrodynamics. I am especially indebted to Rosemary, Al, Bill, and Charlie for numerous discussions, many (spontaneous) calculations, and most of all for their friendship and patience. This research has been encouraged and supported by Dick Fortner, Hal Graboske, and Mark Eckart.

These studies have also been due the successful collaboration with KMS Fusion, Inc. In particular, I would like to thank George Charatis for his friendship, 
guidance, and for sharing his extensive experience in research. I would like to thank Gar. Busch, David Ryoo, David Sullivan, John Brundage, Randy Johnson, Rick Masters, Max Byers, Bill Lawrence, Jeff Stiegman, Bob Schroeder, Chet Shepard, Ed Gabl, Alma Williams, Dave Steinman, John Ruppe, and Virgil Caldwell for their expert technical assistance in successfully executing these complex experiments and for making my extended visits to Ann Arbor both pleasant and constructive.

I would also like to thank Dennis Carr, Tom Stack, Don Duerre, Don Nelson, Jay Gier, Doug Norman, Rex Booth, Phil D'Antonio, Don Burns, and Roger O'Brien for their superior technical assistance in support of this work. I am especially grateful to Dennis for his physical insight, innovative ideas, and plain hard work in helping me design, build, and field the FCXS instrument and complex microdot targets.

Finally, I would like to thank my family and friends for their boundless support throughout these many years. We can all joyously exclaim that it is all "pau!" 


\section{Table of Contents}

Page:

Title Page i

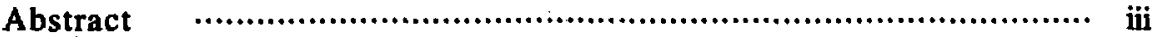

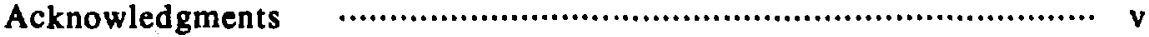

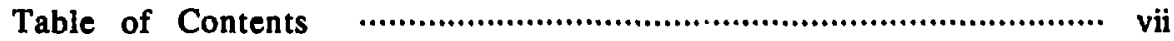

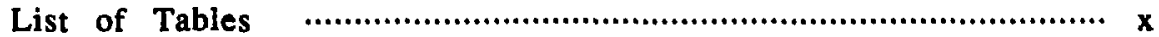

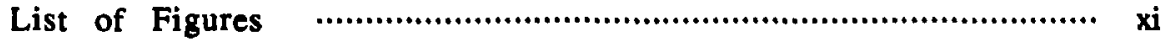

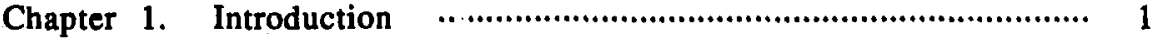

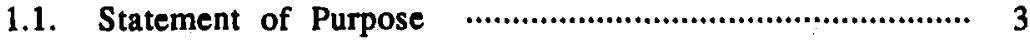

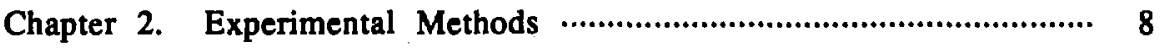

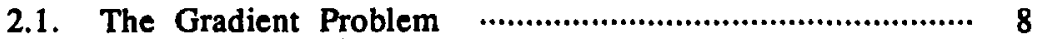

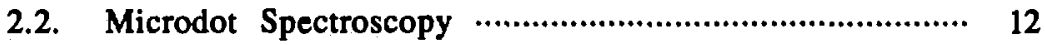

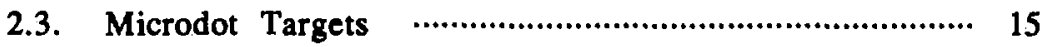

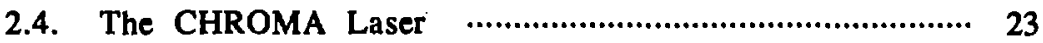

2.5. Simultaneously Space and Time Resolved Diagnostics $\ldots . . . . . . . . . . . \quad 31$

2.5.1. Framing Crystal X-ray Spectrometer (FCXS) ….......... 34

2.5.1a. The Gated Microchannel Plate Framing Camera ...... 37

2.5.1b. The Auston Switch Transmission Line Pulser ........ 43

2.5.1c. Other Important Features of the FCXS $\ldots . . . . . . . . . .55$

2.5.2. X-ray Streaked Crystal Spectrograph (XSCS) …...........6 60

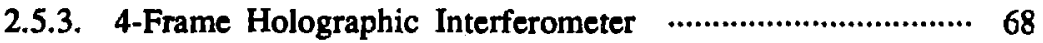

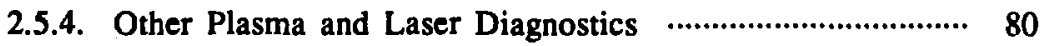

Chapter 3. Important Properties of Laser-Produced Plasmas $\quad$....................... 82

3.1. A General Description of Plasma Models $\ldots \ldots \ldots \ldots \ldots \ldots \ldots \ldots \ldots \ldots . . . \ldots 2$ 
3.2. Collisional Radiative Models …........................................ 85

3.2.1. "RATION" Collisional Radiativc Equilibrium Model …... 90

3.3. Radiation Trapping Effects ….................................. 102

3.4. Important Plasma Parameters

Chapter 4. Density Variation of Line Intensity Ratios in He-like and

Ne-like Ions

4.1. The Resonance to Intercombination Line Ratio: Density

Variation of $n=2$ Lines in He-like Ions ….......................... 120

4.1.1 The Non-Stationary Density Dependence of the

Resonance to Intercombination Line Ratio: A

Quasi-Steady State K-Shell Model

4.2. Density Sensitive High-n He-like Resonance Lines

4.3. The Electric Quadrupole to Resonance Line Ratio: Density

Variation of $(n=2)$ to $(n=3)$ Lines in Ne-like Ions …................. 161

4.3.1 The "NELI" 37 Level Collisional Radiative Model …......... 163

4.3.2. Density Sensitive Metastable Levels in Ne-like

Mo ${ }^{32+}$ Ions

4.3.3. Steady State, 89 Level Ne-iike Collisional

Radiative Model

4.3.4. Recombination and Ionization Effects on the Electric

Quadrupole and Resonance Lines in Ne-like $\mathrm{Mo}^{32}+\ldots \ldots . . . . .188$

Chapter 5. K-Shell Spectroscopy Experiments …................................. 195

5.1. Description of the Experiments …................................. 198

5.1.1. XSCS Aluminum K-shell Data ….......................... 201

5.1.2. FCXS Aluminum K-shell Data …......................... 206

5.2. Measurement of the Electron Temperature Profiles: Temperatures 
Determined Via the Free-Bound Recombination Continuum 212

5.2.1. Comparisons to 2D Hydrodynamic Simulations 221

5.3. Temperature Dependence of $\mathbf{K}$-shell Line Intensities 226

5.3.1. K-shell Resonance Line Ratios 226

5.3.2. Dielectronic Satellite to Resonance Line Ratios 228

5.4. Experimental Line Intensity Ratio Temperatures 232

5.5. Density Sensitive He-like Lines: Results and Discussion 239

5.5.1. A Quasi-Steady State Analysis: Recombination and Excitation Effects on $\mathbf{K}$-shell Lines 248

5.6. Measurement of Inverted High-n Resonance Line Intensity

Ratios in He-like Ions 254

5.7. Spatially Resolved, Time-Integrated Measuremerits ................... 266

5.8. Summary 269

Chapter 6. L-Shell Spectroscopy Experiments ….................................. 272

6.1. Description of the L-Shell Experiments ….......................... 275

6.2. Estimates of the Electron Temperature …............................. 291

6.3. Estimates of the Line Opacity of the L-Shell Plasmas .................. 297

6.4. The " $x$ " Electric Quadrupole to "3A" Resonance Line Ratio:

Results and Discussion ............................................... 300

6.4.1. The "3G" to "3A" Resonance Line Ratio ………........... 310

6.4.2. Possible Short Wavelength Lasing Transitions

Between Metastable Levels in Neon-like Ions ….................... 313

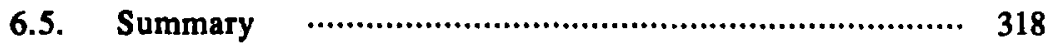

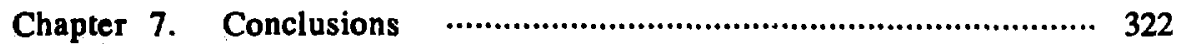

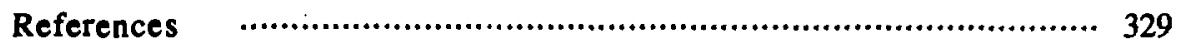




\section{List of Tables}

Page:

Tahle 1. Typical atomic (hydrogenic) collisional and radiative rates.

Table 2. Collisional transition rate coefficients for the $n=2$ Ele-like levels.

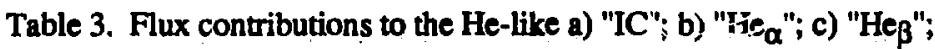

d) "Hey"; and e) "Hes" excited state level populations due to

recombination processes calculated using the quasi-steady state

Al model.

Table 4. Ne-like $\mathrm{Mo}^{32+}$ and $\mathrm{Ag}^{37+}$ electric dipole and quadrupole configurations, wavelengths, and radiative decay rates.

Table 5. Collisional and radiative rates and (\%) fluxes feeding the a) " $x "$;

b) " $3 A$ "; and c) " $3 G$ " excited states calculated using the 37 level

"NELI" model for $\mathrm{Mo}^{32+}$.

Table 6. Recombination and collisional ionization rate coefficients for the Ne-like $\mathrm{Mo}^{32+}$ " $\mathrm{x}$ ", "3A", and " $3 G$ " excited state levels calculated using the quasi-steady state, 89 level Ne-like model.

Table 7. Target shot parameters.

Table 8. Estimates of the L-shell line opacities. 


\section{List of Figures}

Page:

Figure 1. X-ray pinhole photographs of typical laser-produced planar target plasmas.

Figure 2. Contour plots of the electron density and temperature profiles calculated by a 2D "LASNEX" simulations for a laser-irradiated

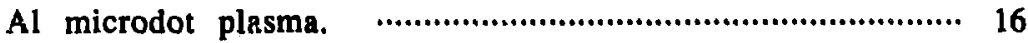

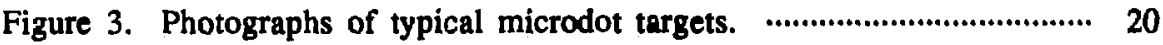

Figure 4. A schematic diagram of the full CHROMA laser system. ................. 24

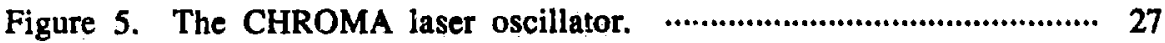

Figure 6. A typical stacked, 1.0 ns wide, CHROMA laser pulse. ................. 29

Figure 7. The experimental set-up in the CHROMA target chamber. ................. 32

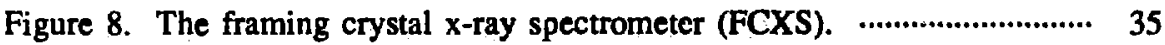

Figure 9. The gated microchannel plate (MCP) framing camera. ................... 38

Figure 10. The Auston switch transmission line pulser. ............................. 44

Figure 11. Typical scope traces of fast, high voltage Auston switch pulses. ....... 46

Figure 12. Scope traces of poor Auston switch pule j. ............................... 51

Figure 13. Aluminum K-shell data measured with the FCXS. ........................ 54

Figure 14. Photographs of various FCXS components. .......................... 57

Figure 15. The $x$-ray streaked crystal spectrograph (XSCS). $\ldots \ldots \ldots \ldots \ldots \ldots \ldots \ldots \ldots . . . \ldots 1$

Figure 16. Aluminum K-shell data measured with the XSCS. ........................ 65

Figure 17. Holographic interferograms of an expanding aluminum

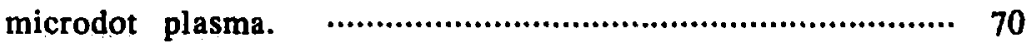

Figure 18. Schematic diagrams of the multi-framed holographic interferometer: $\quad \cdots \quad 73$

Figure 19. Aluminum microdot electron density (axial) profiles. ..................... 76 
Figure 20. A two dimensional density profile for a typical Al microdot plasma. … 78

Figure 21. Various "RATION" (CRE) model line intensity ratio predictions. …... 94

Figure 22. The optical depths predicted by the "RATION" (CRE) model. ……... 106

Figure 23. The predicted geometrical divergence of the plasma flow. ……......... 115

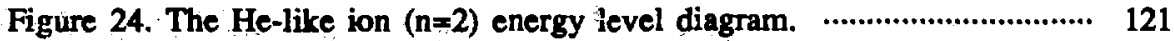

Figure 25. The predicted density dependence of the various He-like $(n=2)$. transitions:

Figure 26: A schematic energy level diagram for the quasi-steady state

K-shell model.

Figure 27. The density dependence of the aluminum " $\alpha$ " line ratio for pure recombination and pure direct excitation population kinetics from the quasi-steady state K-shell model.

Figure 28. A comparieon of the CRE, purely recombining, and purely direct excitation " $\alpha$ " line ratio density dependence.

Figure 29. A comparicon of the "He// $\mathrm{He}_{\beta}$ " and "Heg/He $\beta$ " line ratios

for an $\mathrm{Al}$ plasma populated only via recombination pracesses.

Figure 30. A comparieon of the "Heg/Hes" and "Heg/He $\beta$ " line ratios

for a plasma pepulated via direct excitation only.

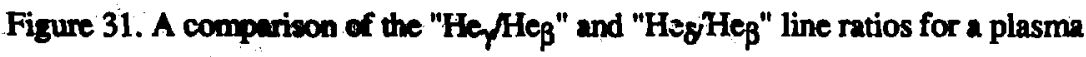
populated by recombination and excitation at $100 \mathrm{eV}$ and $200 \mathrm{eV}$.

Figure 32. A schematic diagram of the Ne-like $(n=3)$ energy level diagram. 165

Figure 33. The density dependence of Ne-like lines predicted by the

37 level "NELI" model.

Figure 34. The density dependence of the Ne-like electric quadrupole to resonance line ratio, " $x / 3 A$ ", predicted by the "NELI" model for $\mathrm{Mo}^{32+}$ and $\mathrm{Ag}^{37+}$. 
Figure 35. The density dependence of the Ne-like resonance line ratio,"3G/3A", predicted by the "NELI" model for $\mathrm{Mo}^{32+}$ and $\mathrm{Ag}^{37+}$.

Figure 36. A comparison of the Ne-like electric quadrupole to resonance line ratio, " $x / 3 A$ ", predicted by the "NELI" model and by a more detailed 89 level model.

Figure 37. A comparison of the $\mathrm{Ne-like} \mathrm{"} x / 3 A^{\prime}$ and " $3 G / 3 A$ " line atios for a recombining plasma and a direct Excitation plasma (89 level model). …. 192

Figure 38. Densitometer scans of typical XSCS aluminum spectra. 203

Figure 39. Densitometer scans of typical FCXS aluminum spectra. 208

Figure 40. Typical digitized FCXS Al spectra showing the H-like free-bound recombination continuum for two different regions in space/time. 216

Figure 41. A comparison of experimentally measured and LASNEX predicted time resolved temperature profiles for an aluminum microdot plasma.

Figure 42. A comparison of experimentally measured and LASNEX predicted time resolved density profiles for an aluminum microdot plasma

Figure 43. A comparison of electron temperature profiles determined from the free-bound recombination continuum and from various line intensity ratio techniques.

Figure 44. The CRE " $\alpha$ " line ratio density profiles for an $\mathrm{Al}$ plasma. ................ 240

Figure 45. The CRE "Hep/IC" line ratio density profiles for an Al plasma. …...... 244

Figure 46. The CRE "He $/(\mathrm{IC}+\mathrm{mn}+\mathrm{st})$ " line ratio density profiles for

an Al plasma.

Figure 47. The CPE " $\alpha$ " line ratio density profiles for a 10\% Al plasma. ........... 249

Figure 48. The " $\alpha$ " line ratio density profiles for a purely recombining or pure excitation plasma (10\% Al microdot plasina).

Figure 49. Simultaneous space and time resolved Al spectra exhibit 
inversions in the high-1: line intensities (He-like and H-like)

at late times in the corona.

Figure 50. A comparison of measured and predicted (quasi-steady state

recombination model) high-n He-like resonance line intensity ratios as a

function of the electron density.

Figure 51. Spatially resolved, time integrated line ratio density and

temperature profiles.

Figure 52. FCXS data for Shot $\$ 8502$, a Mo/Mg bull's-eye target shot. ............. 277

Figure 53. Densitometer scans of the FCXS data from Shot $\$ 8502$. ….............. 279

Figure 54. FCXS data for Shot $\$ 8519$, a $100 \mu \mathrm{m}$ Ag microdot target shot. ......... 285

Figure 55. Densitometer scans of the FCXS data from Shot $\# 8519 . \quad \ldots . . . . . . . . . . . . .287$

Figure 56. Electron density profiles for the Mo/Mg plasma, Shot $\$ 8502$ and the Ag dot plasma, Shot $\# 8519$.

Figure 57. The electron temperature is estimated using the CRE Mg resonance line ratio for the $\mathrm{Mo} / \mathrm{Mg}$ bull's-eye plasmas.

Figure 58. FCXS spectra representing a plasma region $47-94 \mu \mathrm{m}$ from the target surface for $230-480 \mathrm{ps}$ for the $\mathrm{Mo}^{32+}$ and $\mathrm{Ag}^{37+}$ plasmas. … 302

Figure 59. A compurison of experimentally measured and theoretically predicted density dependence of the " $x / 3 A$ " line ratio for Ne-like Mo.

Figure 60. Density profiles using the Ne-like Mo " $x / 3 A$ " line ratio are compared with interferometry density profiles. 306

Figure 61. A comparison of experimentally measured and theoretically predicted density dependence of the "3G/3A" line ratio for Ne-like Mo.

Figure 62. Estimates of gains for short wavelength transitions between metastable levels in $\mathrm{Ne}-$ like $\mathrm{Mo}^{32+}$ and $\mathrm{Ag}^{37+}$ ions. 


\section{Chapter 1. Introduction}

The physics of multiply charged ions in high-density, high-temperature plasmas has become a very active and fertile research area in recent years. These plasmas can be either astrophysical sources such as solar flares, or more often laboratory sources such as laser-produced plasmas, vacuum sparks, exploding wires, gas-puffed z-pinches, or magnetic fusion devices. In the past 15 years, high-powered lasers have increasingly been used as a research tool for studying dense plasmas. Besides providing an excellent laboratory source to produce and study highly ionized atoms, laser-produced plasmas have been the focus of several important rew applications including inertial confinement fusion [Nuckolls et al. (1972)], bright $x$-ray sources for backlighting, $x$-ray radiography, and microlithography [for examole Tomie et al. (1987); O'Neill et al. (1987)], and recently as the medium for short wavelength lasing schemes [Matthews et al. (1985; 1987a: 1987b); T.N. Lee et al. (1987); MacGowan et al. (1987)]. The development and success of these new research areas are dependent on the detailed and accurave characterization of these high-energy plasmas. High quality data is needed to develop and test the sophisticated atomic and hydrodynamic models which are used to simulate the complex microscopic atomic physics as well as the thermal and radiation transport processes in the plasma.

In recent years, research has centered on spectroscopic studies of the x-ray emission prodused by highly charged ions in hot, dense plasmas to test specific atomic or plasma physics processes which influence the population kinetics of these ions. Alternatively, these measurements can be used to diagnose the local conditions of the plasma such as the electron density, temperature, and charge state distribution. Experimentally, the $x$-ray emission spectra is often studied as a function of the electron density, temperature, or charge state distribution and tested against detailed theoretical atomic model predictions. In the present work, I have chosen to examine 
the density dependence of metastable level populations and $\mathrm{x}$-ray line intensities in helium-like and neon-like ions in dense plasmas. The density dependence of various line intensities is primarily due to the effect of high frequency electron-ion collisions on metastable levels and can be used to extract detailed information concerning the detailed population kinetics and level populations of these ions.

These density dependent variations are most clearly seen in high-powered, laser-produced plasmas. A unique problem associated with laser-produced plasmas are the rapid and extreme spatial and temporal gradients which must be clearly resolved to extract meaningful information about the plasma. This introduces an additional problem of fully characterizing the laser-produced plasma and separating the complex hydrodynamic effects from the atomic physics being investigated. Therefore, appropriate refinements to standard experimental techniques and plasma diagnostic methods are required in order to clearly examine the derisity dependent line emission produced by ions in dense, laser-produced plasmas. These new methods provided simultaneously space (all 3 spatial dimensions), time, and spectrally resolved data. The time resolved electron density and temperature profiles were simultaneously and independently measured, thus making it possible to obtain $n$ clex measurement of the density dependence of various spectral line intensities. In particular, I measure the density dependence of the " $\mathrm{He} / \mathrm{KC}$ " ratio and the high-n "He/ $\mathrm{He}_{\beta}$ " and "Heg/Hep" resonance line intensity ratios in helium-like aluminum. I have also measured density variations of the " $x / 3 \mathrm{~A}$ " electric quadrupole to dipole-sllowed resonance line ratio and the " $3 \mathrm{~A} / 3 \mathrm{G}$ " resonance line ratio in neon-like molybdenum and silver ions. The density dependent $K$-shell line ratios were compared to predictions based on standard, collisional radiative equilibrium plasma models and on a new, quasi-steady state atomic model. Similarly, the density dependence of the neon-like line intensity ratios have been compared to two 
detailed, steady state, collisional radiative neon-like models. These studies provide much insight into the detailed collisional coupling between metastable excited state levels, in the various metastable level populations, and in the precise atomic population kinetics influencing the excited state levels of ions in dense, laser-produced plasmas. The results of these new measuremenis and tests of atomic theory are presented and discussed along with suggestions for future studies.

\subsection{Statement of Purpose}

In this thesis, I describe measurements of density dependent line intensity ratios in helium-like and neon-like ions in the underdense (coronal) region of laser-produced plasmas. In order to clearly study the detailed atomic physics of this phenomena and to isolate it from hydrodynamic gradient effects, several new methods to measure and characterize laser-produced plasma were necessary. In Chapter 2, I describe the experimental techniques used in this work which allowed for the measurement of simultaneously space and time resolved $\mathrm{x}$-ray emission spectra from a variety of laser-irradiated torgets for the very first time. This discussion includes a description of the planar microdot targets as well a brief review of various target manufacturing methods. A very important aspect to these experiments was the invention and use of new simultanecusly space and time resolved instruments: a framing crystal x-ray spectrometer (FCXS), a X-ray streaked spectrograph with a spatial imaging slit (XSCS), and a multiple frame holographic interferometer. This is the first time such a sophisticated amy of instruments have been fielded together to siudy laser-produced plasmas. These instruments are described in detail in Chapter 2.

In Chapter 3, I describe some of the standard equilibrium plasma models that are often used to interpret the spectral data measured from typical laboratory plasmas. The majority of this work focussed on laser-irradiated aluminum targets which 
produce K-shell (hydrogen-like, helium-like, and lithium-like) $\mathrm{x}$-ray emission spectra. I briefly outline a K-shell atomic model often used to interpret the $\mathrm{x}$-ray spectral data from dense laboratory plasmas. This is a Collisional Ea äative Equilibrium, "CRE", code called "RATION/RATSHOW/SPECTRA" by R. Lee et al. (1984) which allows the user to compare experimental to theoretical line ratios and extract specific plasma parameters as to the electron temperature and density based on a CRE steady-state ionization distribution. A brief review of plasma opacity effects as well as important plasma parameters that were considered during these experiments are also presented.

In Chapter 4, I present a more detailed discussion of specific density sensitive line intensity ratios which have been investigated in this work. These are the resonance to intercombination line ratio and the high-n resonance line ratios in helium-like ions. I describe the density dependence of each line ratio and present the standard, stationary methods used to interpret the density dependence of these line intensity ratios. I also present a new, quasi-steady state K-shell model which allows for detailed analysis of specific line intensity ratios without having to assume an equilibrium ionization distribution. This non-stationary mudel is successfully tested in the following chapter for both the resonance to intercombination line ratio as well as the line ratio between high-n He-like resonance lines. For neon-like ions, I examine the electric quadrupole to electric dipole-allowed resonance line intensity ratio. The density dependence of this line ratio is discussed and two quasi-steady state neon-like models are described. These collisional radiative neon-like models correctly account for the density dependence of the neon-like electric quadrupole to electric dipole resonance line intensity ratio.

In Chapter 5, I describe my experiments to study the K-shell emission spectra from laser-produced aluminum plasmas. The K-shell emission was measured from a range of plasmas conditions and from two types of aluminum microdot target 
concentrations used to vary the plarma opecity of the aluminum emission lines. The time-resolved electron temperature profiles have been determined from the slope of the hydrogen-like free-bound recombination continuum measured using the framing crystal spectrometer. The electron density profiles were simultaneously measured using multi-frame holographic interferometry. The time resolved electron temperature and density profiles are compared to two dimensional "LASNEX" hydrodynamic simulations to measure the effects of laser absorption and heat transport in the laser plasma corona. Although these comparisons are very preliminary, they do demonetrate a new cepability to expand our understanding of both the atomic physics of dense plasmas and the hydrodynamics of high-powered laser-plasma inveractions. The simultuneously space and time resolved K-shell emission spectra were analyzed to study the density and temperature dependence of the measured lines with the predictions of the standard, CRE theoretical atomic model as well as the more detriled, quasi-steady state model described in Chapters 3 and 4, respectively. In particular, I examine the density dependence of the helium-like resonance to intercombination line ratio and between the high-n resonance lines in He-like ions. The CRE model fails to predict the observed density and temperature dependence of the $x$-ray epectra correctly and this clearly illustrates the failure of standurd, equilibrium line matio methods to properly account for the non-stationary nature of these laser-produced plasmas. On the other hand, both the resonance to intercombination line ratio and the high-n He-like resonance line ratios are successfully modeled by the quasi-stendy state, recombining model. This is the first clear and successful demonstration of the detailed modeling of a laser-produced plasma and represents a significant advancement in our understanding of these plasmas.

In Chapter 6, I describe the x-rny spectroscopy experiments conducted on molybdenum $(Z=42)$ and silver $(Z=47)$ microdot turgets. These were an extension 
of the K-shell spectroscopy experiments described in Chapter 5. The physics of high- $Z$ ions stripped to the neon-like ionization state has drawn considerable interest due to the recent development of short wavelength lasing schemes based on these plasmas. These L-shell experiments employed the same simultaneously space and time resolved techniques to provide clear, precise spectral data to compare against available theoretical atomic models. The molybdenum targets were mixtures of magnesium and molybdenum. The $\mathrm{K}$-shell spectra emitted by the magnesium ions allowed a rough estimate of the electron temperature of the plasma. The main goal of this work was the investigation of density effects on metastable levels in neon-like ions. In particular, I have focussed on the $\left.\left[1 s^{2} 2 s^{2} p^{6} 3 d(1 / 2,5 / 2)\right]=2\right]$ to $\left.\left[1 s^{2} 2 s^{2} 2 p^{6}(0,0)\right]=0\right]$ electric quedripole (E2) transition (referred to as " $x$ " in the remainder of this dissertation) and the $\left[1 s^{2} 2 s^{2} p^{6} 3 p(1 / 2,3 / 2) J=1\right]$ to $\left.\left[1 s^{2} 2 s^{2} 2 p^{6}(0,0)\right]=0\right]$ dipole-allowed " $3 A^{\prime}$ resonance line transition in neon-like $\mathrm{Mo}^{32+}$ and $\mathrm{Ag}^{37+}$ ions. The "x" $\mathrm{E} 2$ transition suggests population inversions between upper excited levels in neon-like ions which correspond to $\mathrm{x}$-ray transitions below the carbon K-edge. Both the " $x$ " and the "3A" transitions are predicted to be sensitive to variations in the electron density by steady state collisional radiative neon-like atomic models. I test the density sensitivity of these transitions by comparing their theoretical to experimental line intensity ratios over a range of independently measured electron densities. Both Ne-like models successfully account for the density dependence of the Ne-like " $x$ " to "3A" line intensity ratio. This provides more evidence of our increased understanding of the complex physical processes ongoing in these dense, laser-produced plastnas.

This work is summarized in Chapter 7. These experiments have provided a wealth of new spectral data which is relatively free of spatial and temporal gradients which in the past has prevented clear comparisons to theoretical atomic model predictions. 
More importantly, this work has demonstrated new techniques which can be applied to further understand both the detailed microscopic atomic physics ongoing in these high-energy plasmas as well as the complex laser-matter interaction which is of fundamental importance to a wide community of researchers dealing with these types of laboratory plasmas. A discussion on improvements to these experiments is included in this chapter as well as a few suggested ideas on future laser-plasma experiments. 


\section{Chapter 2. Experimental Methods}

In this chapter, I describe the instrumentation and target design used in these experiments to measure density dependent spectroscopic effects. In laser-produced plasmas, the ions being studied are subject to severe space-time hydrodynamic gradients. In order to isolate the density dependence of the ionic spectral features from the effects of these gradients, it is neccessary tu: i) perform simuitaneously space and time resolved measurements of the emission spectrum, and ii) fully characterize the laser-plasma source to determine its electron density and temperature profiles in all 3 spatial dimensions and in time. This is a challenging problem that historically has limited the accuracy of previous studies of both atomic physics and hydrodynamic phenomena in dense plasmas. The solution to this problem involves the invention of new $\mathrm{x}$-ray imaging instruments and the careful target design which are described in below.

\subsection{The Gradient Problem}

Attempts to minimize these gradient effects on $x$-ray spectral data have been the focus of recent studies. Some of the first spatially resolved $x$-ray data from laser-produced plasmas was reported by Boiko et al. (1975). They used a $65 \mu \mathrm{m}$ side imaging slit with a convex mica Bragg diffraction $\mathrm{x}$-ray crystal spectrometer to study plasmas formed by Nd laser pulses focused to $5 \times 10^{14} \mathrm{~W} / \mathrm{cm}^{2}$ on slabs of material ranging from magnesium to vanadium. Their measurements revealed the spatial profiles of He-like resonance lines, intercombination lines, and satellite lines. Spatial resolution was attained by using a narrow imaging (entrance) slit. The slit is narrow enough to ensure that for a given wavelength $\lambda$, the recorded $x$-ray emission originates from a well defined, small region of plasma. The main drawback to this 
technique is the low signal-to-noise ratio of the recorded spectral data. This is caused by the small number of photons that reach the film due to the small acceptance solid angle that is defined by the narrow entrance slit. Detailed discussions of these measurements and data are presented in a series of articles by Boiko et al. (1978; 1979a; 1979b; 1983; 1984). Further efforts to increase the spatial resolution of spectral studies of laser plasmas have been conducted by a host of researchers including Yaakobi and Nee (1976), Bhagevatula and Yaakobi (1978), Key et al. (1977; 1980), Lunney et al. (1979), etc. Recent studies by Alaterre et al. (1984) and Audebert et al. (1984a; 1984b) have yielded very high spatially resolved (few microns) spectral data using a knife edge technique. Their techniques improve the signal-to-noise ratio of the data. The main experimental difficulties with this technique includes the need to deconvolute the measured data ard the complicated precision alignment of the imaging knife edge for each shot.

Spectroscopic studies of laser-produced plasmas were greatly improved by the introduction of spot spectroscopy (microdot targets) techniques first used by Hertst et al. (1981; 1982) and Burkhalter et al. (1983). The material of spectroscopic interest was placed in a localized spot typically using vacuum plating techniques. This spot (or dot) of "seed" material was mounted on a low $\mathbf{Z}$ substrate, such as a thin plastic sheet. A heating laser beam was focussed onto the dot target overfilling the target. The resulting plasma is one in which a plume of "seed" plasma is allowed to expand away from the target surface (along the heating laser axis) and remains collisionally confined (radially) by the surrounding, relatively transparent substrate plasma. Spatially resolved spectrometers were positioned to resolved the axial direction of the plasma. Transverse (radial) gradients in the plasma are minimized in this type of target because the size of the "seed" plasma column is smaller than the scale length for these gradients. This allowed for spatially resolved (although time-integrated) measurement of $x$-ray emission from fairly localized regions of 
laser-produced plasmas. The small spatial extent (radial) of the plasma also offered some control of the target opacity.

The first time resolved $\mathrm{x}$-ray spectra from laser-prc duced plasmas were obtained by Key et al. (1980) using an $x$-ray streak camera coupled to a crystal spectrometer. In these first measurements of $\mathbf{K}$-shell resonance, intercombination, and satellite lines, Key and his coworkers reported on the temporal profiles of these lines and noted the relationship between the spatial and temporal profiles of the luminous region of the expanding plasmas. The limitation of this diagnostic technique is that the instrument typically views the entire plasma and dnes not yield any spatial information. Attempts to obtain course spatial resolution with these streak camera instruments have had only limited success. Kauffman et al. (1984) reported on measurements of the time resolved emission spectra fi sm silicon seeded $\mathrm{CH}$ and $\mathrm{PbO}$ plasmas. Coarse spatial resolution was obtained using a sharp knife edge to mask off a wide region of the plasma plume. In the experiments described here, a refined version of this streaked $x$-ray spectrometer using a pair of slits to obtain course spatial resolution was fielded by Charatis et al. $(1986 ; 1987)$ and is described liter in this chapter.

Recently, there have been spectroscopic studies to obtain simultaneously space and time resolved spectril data from laser-produced plasmas in which a thin layer of seed material (typically low-Z to study the $K$-shell ex ission) is used to define a previse space and time interval in the evolving plasma. [Kauffman et al. (1984); Majoribanks et al. (1987); Kilkenny et al. (1980)] This layer of material can be overconted with a layer of plastic. Assuming a known mass ablation rate for the plastic overcoat, one has some control over the time in which the seed material will be heated and become luminous by varying the thickness of the plastic overcoat. Typically both spatially resolved (ime-integrated) and time resolved (spatialiy-integrated) diagnostics are used to measure the emission spectra of the 
plașma.

This technique offers some improvement over previous experiments in providing some measure of space and time resolution. But this technique is also limited due to a number of reasons. Because only a small amount of seed material is used and is luminous for a finite time as the plasma expands, only a small interval of space and time parameter space can be sampled for a particular shot. In onder to do a detailed study of the entire plasma, a whole sequence of identical shots must be studied. This can be difficult experimentally due to fluctuations in experimental conditions (such as in the laser irradiance conditions) which are problematic to these tynes of laser-matter interaction experiments. Secondly, this method assumes the hydrodynamics of the evolving plasma has a simple space-time relationship. This is true only if there aren't any large velocity eradients in the plasma. This is a hydrodynamics problem that must be addressed. The thickness of the seed material layer must be kept thin to provide a well defined spatial and temporal region. This may introduce plasma opacity problems if the plasma is optically thin along a path other than that of the recording spectrometer. This is because the emission lines "escape" through the "thinest" optical path in the plasm. Therefore, use of this type of target to attain space and time resolution must account for this opacity effect. Finally, this technique requires a precise modification of the laser target to be studied. The target must be designed so that this thin layer of thin material behaves properly, in a pressure balance, both radially ane axially. A simple similarity solution can be used to provide an empirical design criteria. [London (1986)] Physically, the construction of these targets may be difficult due to their delicate nature and complex design. 


\subsection{Microdot Spectroscopy}

The posl of the experiments was to masure time resolved spectra from a gradient free (isodense, isothermal) plasm volume. Since it is practically impossible to create such uniform high energy-density plasmas, these experiments used a variation of the spot spectroscopy techniques of Herbst $\alpha$ al. (1981; 1982) and Burkhalter et al. (1983) to measure the x-ray emistion from amall, localized volumes of the laser-plasma produced by irrediexing simple planar microdot turgets. The dot portion of the target was compesed of the element or elements to be studied, mounted on a plastic subetrave. The microdot taret wasthen illuminated by a laser focal spot which overfilled it by a factor of 3 or more. The surrounding carbon plasma collisionally confined the teat ions in the central region of the plasma plume and was transparent to the $x$-ray emiasion being measured. Since the radius of the laser-plasma containing the wat ions is mall compared to gradient scale lengths in the plasma, the remaining axidl grodient conld be readily resolved using simple imaging slit techniques. This technique allows us to see emission from tiny, but gradient free regions of plesme. The tranoverve volume of the emitting plasma was defined by the size of the dot troet and dramatically effects the quality of the meavured spectra. Since the spectrn originames from small, faidy isodense, iscthermal volumes of plasma, the spected lise widths are defined by the finite size of the target (source size). This technique also offers some control over the plasma opacity effects on the oberved line emission by minimizing the plasma length along the line of sight of the epectrographs (perpendicular to the laser axis) and also by offering a means of defining the number of abcorbing ions in the plasma.

In Figure 1, time-integrated $x$-ray pinhole phowographs of a typical planar slab plasma and an $\mathbf{A}$ microilot urget plasm show the great improvement in controlling the radial expansion of the plasm attained by using microdot targets. A detailed, 
Figure 1. X-ray pinhole photographs of typical laser-produced planar target plasmas generated by a stacked, $1.0 \mathrm{~ns}$ wide, $\approx 10 \mathrm{~J}, 0.53 \mu \mathrm{m}$ laser beam focused using a $f / 2.5$ kns show (left) a poorly defined plasma formed by irradiation of an $\mathrm{Al}$ slab target with a tightly focused $(-100 \mu \mathrm{m}$ diameter) laser beam, and (right) a well defined, nearly one dimensional plasma plume formed by overfilling a $100 \mu \mathrm{m}$ diameter Al microdot target using a $250 \mu \mathrm{m}$ diameter laser focal spot. 


\section{0 micron diam.}

\section{Slab Target}

$100 \mu \mathrm{m}$ dim. tocus

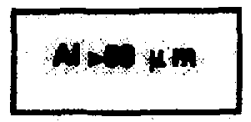

\section{Dot Target}

$250 \mu$ m diem. tocus

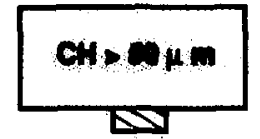

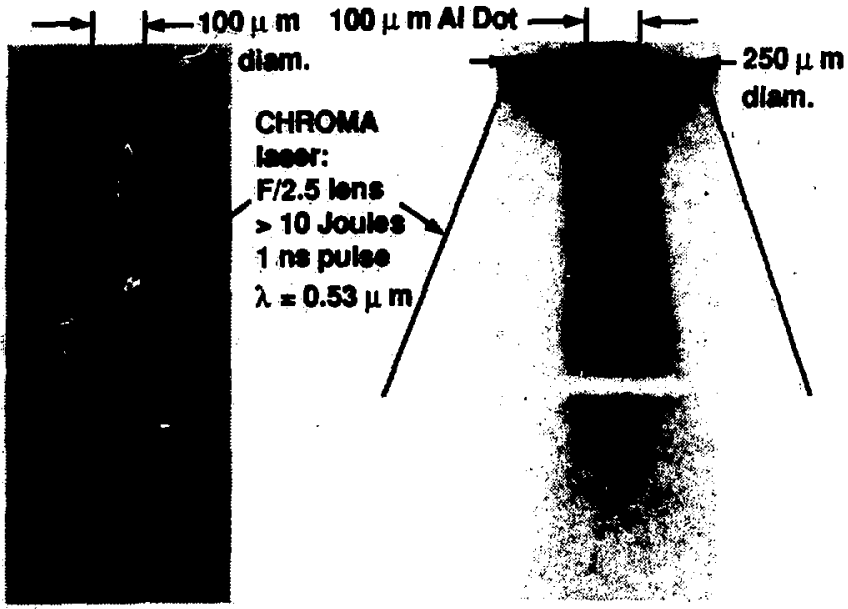

Figure 1 
two dimensional "LASNEX" hydrodynamic simulation of a typical laser heated microdot target is shown in Figure 2. [Cerjan (1988)] The calculations predict nearly flat density and temperature (transverse to the laser axis) profiles with approximately $\pm 10 \%$ variation across the observed area of the expanding microdot plasma and therefore suggest that microdot targets produce plasmas with gradients in only one direction. A comparsion of experimental data to these "LASNEX" simulations is presented in Chapter 5. Temporal gradients were minimized by sampling times much shorter than the heating laser pulse length.

\subsection{Microdot Targets}

Microdot targets were used to study high-powered laser-produced plasmas. This technique was first suggested by Herbst et al. $(1981 ; 1982)$ and is now widely used to study laser-produced plasmas. [For example, see Burkhalter et al. (1983); Alaterre et al.(1984), Audebert et al. (1984a; 1984b); Bailey et al. (1986); Gauthier et al. (1983); Zigler et al. (1987).] The basic design of these microdot targets is the placing of a small dot of "seed" material of the element or elements of interest. For these experiments, the dot material was typically aluminum. The dot of material is typically few tens of microns in diameter. The small dot size reduces the source broadening of the measured spectral lines. The opacity of the plasma can be controlled by varying the size of the dot target or by using a mixture of materials for the dot and thus diluting the "seed" element. The total integrated sigral of the dot plasma is proportional to the area of the microdot target (assuming a constant mass ablation rate). For these spectroscopic experiments, very weak dielectronic satellite lines and free-bound continuum were studied. This small signal level problem is magnified due to the small plasma volume studied and due to the simultaneous time and space resolution of the instruments. Therefore a very delicate balance between 
Figure 2. Two dimensional "LASNEX" hydrodynamic simulations [Cerjan (1988)] of a laser-irradiated ( $100 \mu \mathrm{m}$ diameter) Al microdot plasma show that radial variations in the electron density and temperature profiles are approximately $\pm 10 \%$ over the Al plasma. Two sample times are presented: (left) $730 \mathrm{ps}$ and (right) 1200 ps. Axial density values are used throughout the work. "LASNEX" temperature predictions are compared with experimental data in Chapter 5 based on the outer $\mathrm{Al}$ zones (approximately $75 \mu \mathrm{m}$ radius at $730 \mathrm{ps}$ and $100 \mu \mathrm{m}$ radius at later times). Comparisons with $\mathrm{x}$-ray pinhole photographs indicate that the real microdot plasmas remain confined longer (in both space and time) than predicted by the hydrodynamic simulations. 
Figure 2
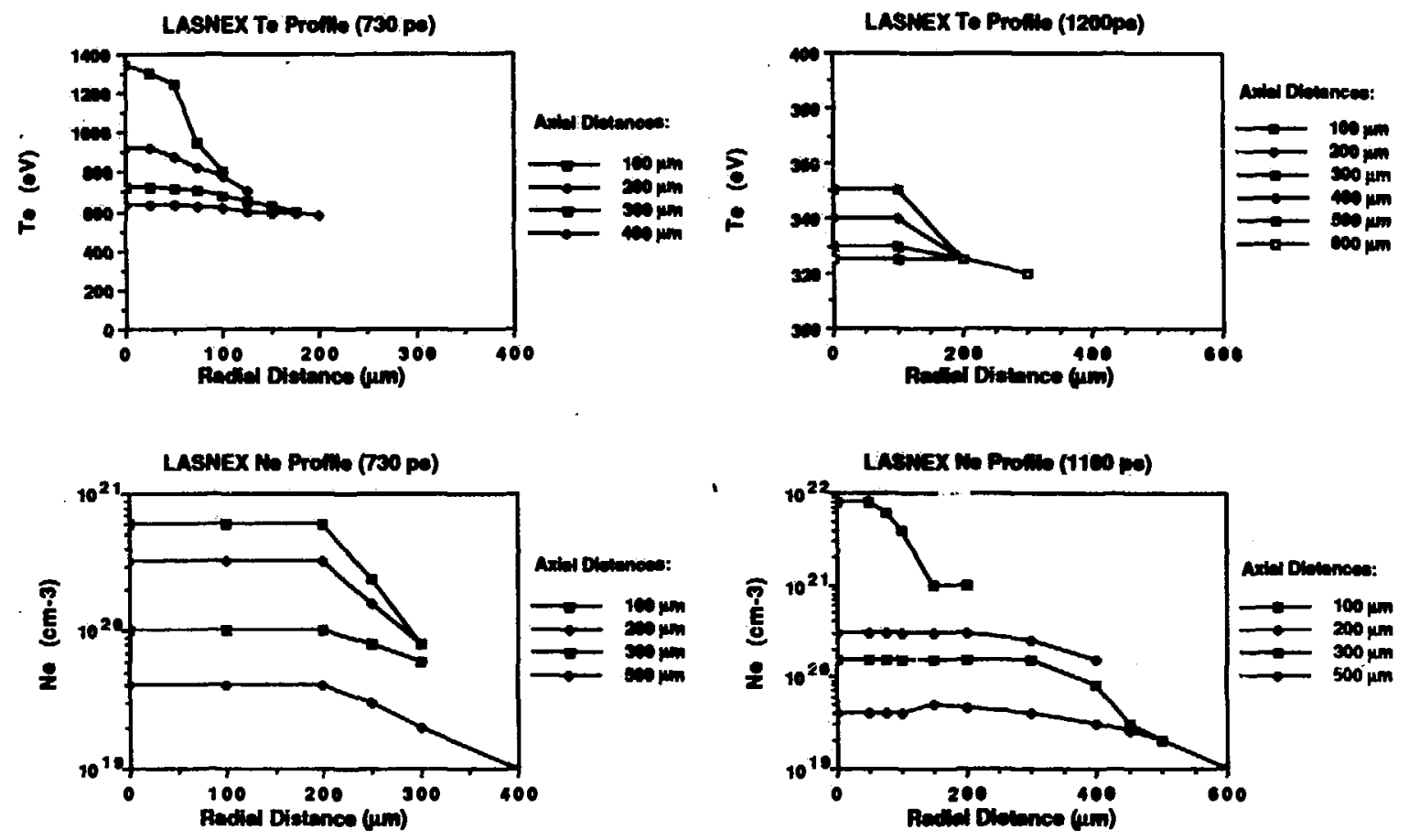
maximizing the emission signal level, controlling the opacity of the target, and minimizing the spectral source size was needed to obtain high quality spectral data. The minimum target size for thesc experiments was determined (empirically) to be $100 \mu \mathrm{m}$ diameter and $>1 \mu \mathrm{m}$ thick for $1 \mathrm{~ns}$ pulses with irradiances of $10^{13}$ to $10^{14}$ of $526.0 \mathrm{~nm}$ laser light. These target design paramet /s were set primarily by the sensitivity of the space and time resolved spectrometers and the overall goals of these experiments. [See Section 2.5.] Typically, these targets were mounted on $125 \mu \mathrm{m}$ thick mylar $\left(\mathrm{C}_{10} \mathrm{H}_{8} \mathrm{O}_{4}\right)$ or polystyrene $(\mathrm{CH})_{\mathbf{x}}$. A much smaller diameter target can be used ( $\$ 10 \mu \mathrm{m}$ diameter) if only $\mathrm{K}$-shell or $\mathrm{L}$-shell resonance lines are to be studied. This would also improve the design of the target by further reducing lateral gradients in the plasma and by reducing the plasma gradient effects in general, thus allowing for a more accurate investigation of isodense, isothermal plasmas.

A variety of methods for fabricating this type of target were utilized. Vacuum deposition (both electrode vapor-plating and ion-sputtering) was used to make many of the targets studied. In this process, a plating mask was mounted flush onto the mylar substrate. Typically, the masks contained an array of assorted microdot hole sizes from 25 to $200 \mu \mathrm{m}$. A set of identical microdot targets of the same thickness and composition over a range of target sizes (diameters) were fabricated at one time. Most of these microdot masks were made from thin silicon wafers that were directionally photochemically etched producing square holes with tapered edges to minimize shadowing due to the walls of the mask. These photochemically etched masks were provided by D. Ciarlo of LLNL. A few of the masks were made of stainless steel with the holes formed using precision microdrills which were then lapped smooth. Aluminum, magnesium, sodium chloride, molybdenum, or silver was then vacuum deposited through the mask to thicknesses of approximately 1-2 $\mu \mathrm{m}$.

Additional targets were made using the vacuum deposition technique including 
sodium chloride/aluminum, molybdenum/magnesium, and silver/sodium fluoride bull's-eyes. These targets were made with a $20-75 \mu \mathrm{m}$ (length along an edge) dot of material coated on top of a 160-200 $\mu \mathrm{m}$ (length along an edge) dot of another material in a two step coating process. The Mo/Mg bull's-eye targets were constructed using the vapor plating technique. The cross section of this bull's-eye target resembles a pillbox. The outer annulus is first coated onto the substrate. It is a simple large dot of one material. The second center dot composed of a second material is then coated on top of the first dot. A precision indexing substrate/mask holder was used to align the target masks. The alignment of the masks was checked using a high powered microscope. For the $\mathrm{Mo} / \mathrm{Mg}$ targets, the Mo concentration was diluted with $\mathbf{M g}$ to form a target that would produce Mo Ne-like resonance lines that were optically thin. A mixture of 1:8 $\mathrm{Mo}$ to $\mathrm{Mg}$ atoms was used for this dot. This was achieved by coating alternating layers of $\mathrm{Mo}(50 \AA \pm 10 \%)$ and $\mathrm{Mg}(400 \AA$ $\pm 10 \%)$ until a thickness of over $1.5 \mu \mathrm{m}(15000 \AA)$ was formed. This was very time consuming and tedious but the composition of these targets was precisely known. A typical Mo/Mg bull's-eye target is shown in Figure 3(left).

Although the vacuum deposition technique was successful in providing a number of microdot targets, there were a few limitations of this method. Not all materials are readily vacuum deposited either because of their high evaporation temperatures or because they disassociate under vacuum or heat. This limits the number of materials (seed elements) that can be used to make these vapor deposited targets. Also, the plating mask must be in close contact to the substrate. Otherwise, the material vapor deposited can leak under the mask and contaminate the surrounding substrate. This would introduce an uncertainty in the size and composition of the microdot target. Also, care must be taken to ensure that the walls of the microdot mask do not obscure the surface producing an irregular target edge. The targets were examined under a high powered 200X microscope. Sample targets were also photographed to 
Figure 3. Photographs of typical microdot targets used in these experiments: (left) a vapor plated $\mathrm{Mo} / \mathrm{Mg}$ bull's-eye $(70 \mu \mathrm{m} / 170 \mu \mathrm{m})$ microdot target (the top dot consists of altemating layers of $50 \mu \mathrm{m}$ of $\mathrm{Mo}$ and $400 \mu \mathrm{m}$ of $\mathrm{Mg}$ to a total thickness of over $1.5 \mu \mathrm{m}$; the lower dot consists of $1.5 \mu \mathrm{m}$ thick $\mathrm{Mg}$; the substrate is $125 \mu \mathrm{m}$ thick mylar), (center) a $10 \% \mathrm{Al}\left(5 \cdot \mathrm{Al}_{2} \mathrm{O}_{3}+75 \cdot \mathrm{C}\right) 100 \mu \mathrm{mD}$ microdot target fabricated by pressed powder techniques (both the dot and $(\mathrm{CH})_{x}$ substrate are $\geq 100$ $\mu \mathrm{m}$ thick), and (right) a SEM photograph of the $10 \% \mathrm{Al}$ target. 


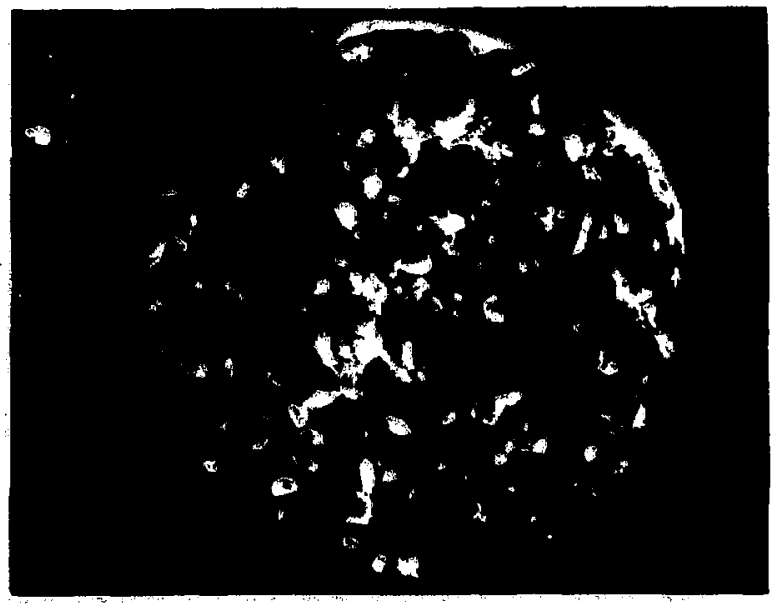

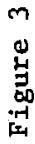
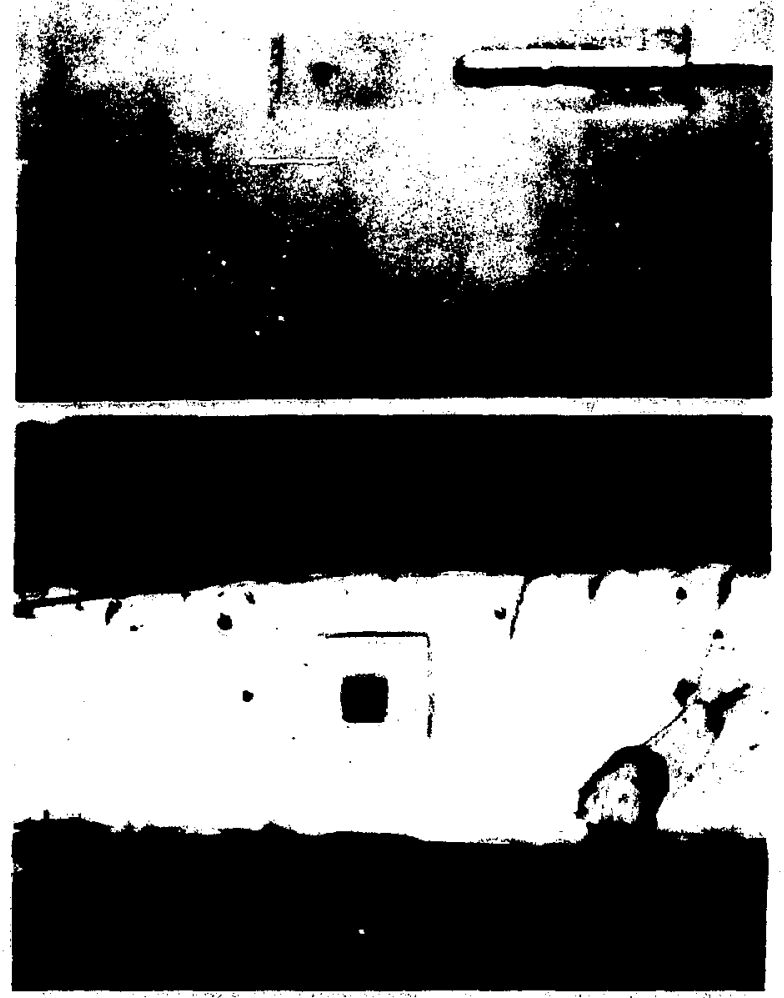
verify the uniformity of the coating. And EDX (electron beam excitation) x-ray mapping was used to test the homogeneity of the targets. [Steinman et al. (1987)] An alternative method was used to make the rest of the microdot targets. This method was developed by D. Steinman et al: (1987) at KMS Fusion. Powders of interest were ground to $<5 \mu \mathrm{m}$ size or smaller using a mortar and pestle then manually pressed into predrilled holes using a fire-polished glass rod. The result is a tightly packed pellet of material embedded in the plastic substrate (polystyrene). The top $25 \mu \mathrm{m}$ is shaved off of the substrate using a mini-lathe with a diamond cutting tool producing a flat, clean surface and well defined target. Again, the targets were examined visually using a high-powered microscope and using EDX x-ray mapping to test for homogeneity. This method offered several advantages over vacuum deposition. A wider range of materials could be used and predetermined mixtures could be readily combined and incorporated into a target. Some of the targets produced by this impacted powder method included $100 \mathrm{umD}$ Al dots and $10 \% \mathrm{AI}$ dots (mixture of 5: $\mathrm{Al}_{2} \mathrm{O}_{3}+75 \cdot$ graphite). A magnified photograph and a SEM view of a 10\% Al dot target are shown in Figures 3(center) and 3(right) respectively. Other targets made using this method include mixtures of $\mathrm{C}_{6} \mathrm{Br}_{6}$ and $\mathrm{MgO}, \mathrm{Al}$ and $\mathrm{NaF}$, etc.

The substrates were then cut to approximately 2 to $3 \mathrm{~mm}$ wide strips and mounted onto a glass stalk or thin wire, then onto a standard KMS target mounts. The width of the target substrate was kept small to prevent edge diffraction effects on the holographic interferometer probe beam. [Busch et al. (1985)] This also prevented the substrate from obstructing the line-of-sight of any of the instruments to the plasma column, especially the heated target surface. The transparent plastic substrate also aided in locating the dot targets. A TV viewing system was used to locate the targets in the target chamber. The system used the CHROMA laser optics and collected reflected and scattered light from a low powered alignment laser beam off 
of the target and relayed it to a TV viewing system. Some of the targets such as the $10 \% \mathrm{Al}$ dots were difficult to locate and a HeNe backlighting set up was used for these targets. A low powered HeNe laser was shined through from the back of the target to backlight it and imaged it onto the TV viewing system. This method proved to be very effective although time consuming in locating and aligning the targets. These microdot targets proved to be a significant improvement over slab targets in providing a nearly one-dimensional laser-plasma source for these spectroscopic studies.

\subsection{The CHROMA Laser}

The experiments were performed using the CHROMA laser facility at KMS Fusion, Inc. in Ann Arbor, Michigan. The CHROMA laser is a Nd:YAG system that can generate about $2.0 \mathrm{TW}$ at $1.05 \mu \mathrm{m}$ in each of two beams. A third beam of approximately $1.0 \mathrm{TW}$ is available for backlighting applications but was not used in this work. A fourth probe beam is used for a multi-frame holographic interferometer which is discussed later in this section. [See Figure 4.] All of these beams are generated in a common actively mode-locked oscillator. This oscillator cavity generates a mode-locked train of short pulses (70-100 ps FWHM) separated by 8.0 ns (defined by the oscillator cavity length). Two of these pulses are switched out of the train by Pockels cell switches. One of the switched out pulses feeds a second oscillator cavity which amplifys it and shortens it to 20 ps FWHM. The shortened pulse is the probe beam which is used for the multi-framed holographic interferometry, for activating the high voltage FCXS Auston switches, and as a timing fiducial for all of the time resolved plasma diagnostics. The second switched out oscillator pulse is sent to a pulse stacker which forms the desired pulse shape to feed the main laser amplifiers. The absolute time interval between the two pulses is 
Figure 4. Schematic diagram or the full CHROMA laser system located at KMS Fusion, Inc., Ann Arbor, Michigan. 


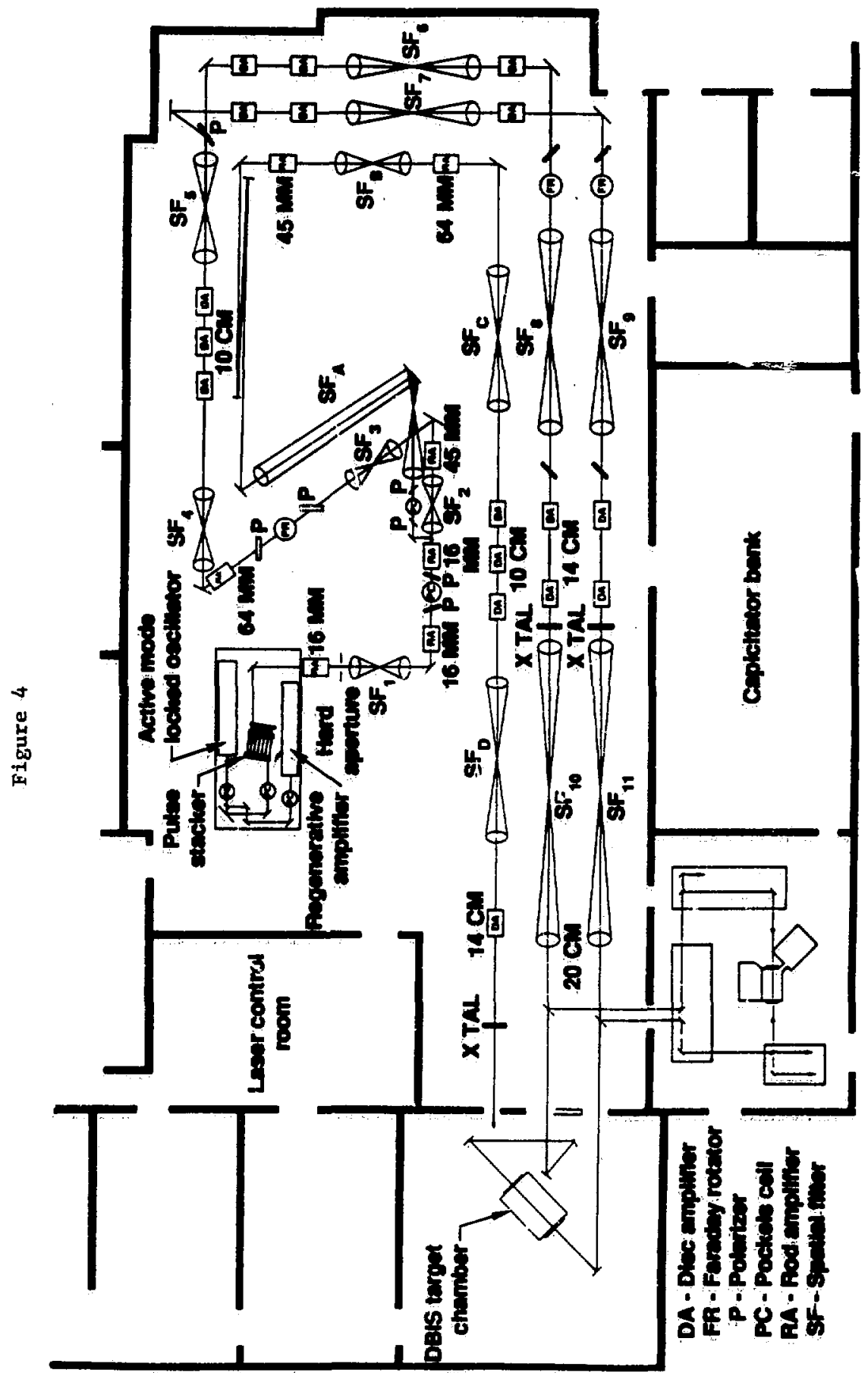


determined by the oscillator (amplifier) savity dimensions. All other timing differences were due only to differences in the optical path lengths of the two beams. The layout of the CHROMA oscillator table showing the active mode-locked oscillawor, the regenermive amplifier, the autocorrelator, and the pulse stacker is shown if Figure 5.

In theac experiments, only one of the main CHROMA laser beams were used to heat the planer turgets. This was the "B" beam at KMS Fusion. Also note that the "DBIS" target chamber was used. The pulse oscillator was tuned to generate approximately 100 ps wide (FWHM) Gauscian pulses. The pulse stacker was set up to add ten pulses of equal amplitude with a spacing of 108 ps which then fed the main amplifiers in chain "B". This generated a fuirly uniform trapezoidal heating pulse with a rise time and fall time of approximately $100 \mathrm{ps}$ and a flat-top of typically 1000 ps. The temporal profile of a typical CHROMA stacked pulse is shown in Figure 6 in which a small fraction of the heating pulse (leakage off of a tuming mirror) was measured uaing an optical streak camera (Hamamatsu C979 Streak Camera). In these experiments, the heating pulse was frequency doubled to 526.0 nm using a KDP Type II crystal. This green pulse is relayed into the target chamber and focussed onto the target using an $f / 2.5$ lens. The $f / 2.5$ lens was adjusted so that the laner beam illuminand the target (located at the center of the target chamber) in the "fir-field" of focus to spot diameters typically $250 \mu \mathrm{m}$ in diameter. The beam energy was typically 5 w0 150 joules of $523.0 \mathrm{~nm}$ light generating beam intensities of $1.0 \times 10^{13} \mathrm{w} .0 \times 10^{14} \mathrm{~W} / \mathrm{cm}^{2}$. Because of the nearly flat-top (temporal) heating pulse, this beam intensity was fairly constant on the target over the duration of the laser pulse.

The laser beam was highly diagnosed by a wide array of laser diagnostics. In addition to the optical streak camera to measure the temporal profile of the heating pulse, an autocorrelator was used to measure the pulse widths of both the initial 
Figure 5. Schemaric diagram of the CHROMA laser oscillator including the main master oscillator, the pulse stacker, and the regenerative amplifier (used to generate the 20 ps probe pulse for the holographic interferometry, the XSCS time fiducial, and activation of the intrinsic Auston switches used to electrically drive the FCXS detector). The main oscillator drives both the main heating laser beams and the probe beams and are synchronized to $\leq 5 \mathrm{ps}$. 


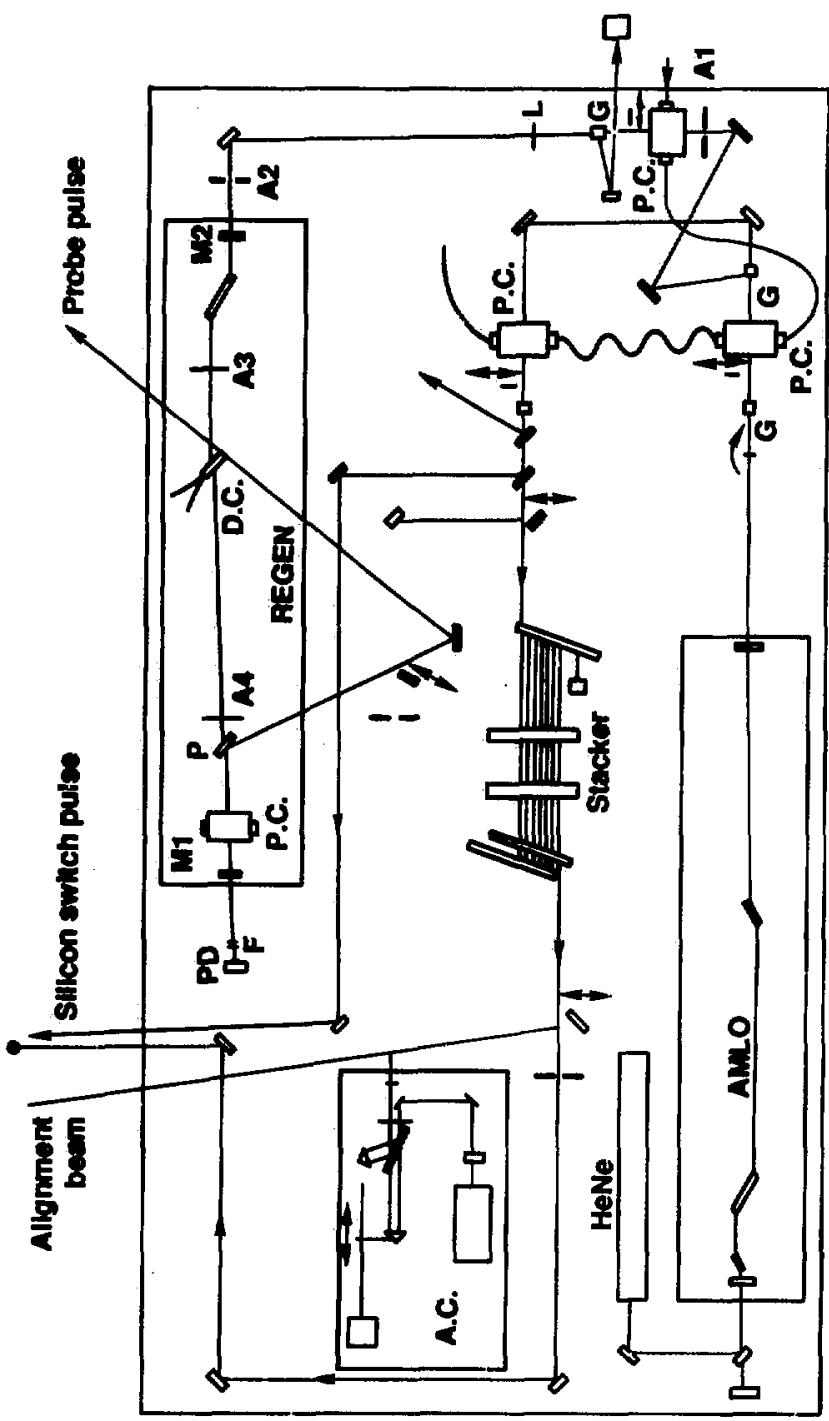

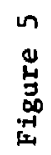


Figure 6. An digitized laser intensity plot measured using an optical streak camera reveals the temporal profile of a typical stacked CHROMA laser pulse generated by the addition of ten 100 ps FWHM pulses with a spacing of approximately 108 ps each. The resulting pulse is nearly trapezoidal with a rise time and fall time of approximately $100 \mathrm{ps}$ and a "flat-top" width of about $1.0 \mathrm{~ns}$. This is the nominal pulse shape used throughout these experiments. 


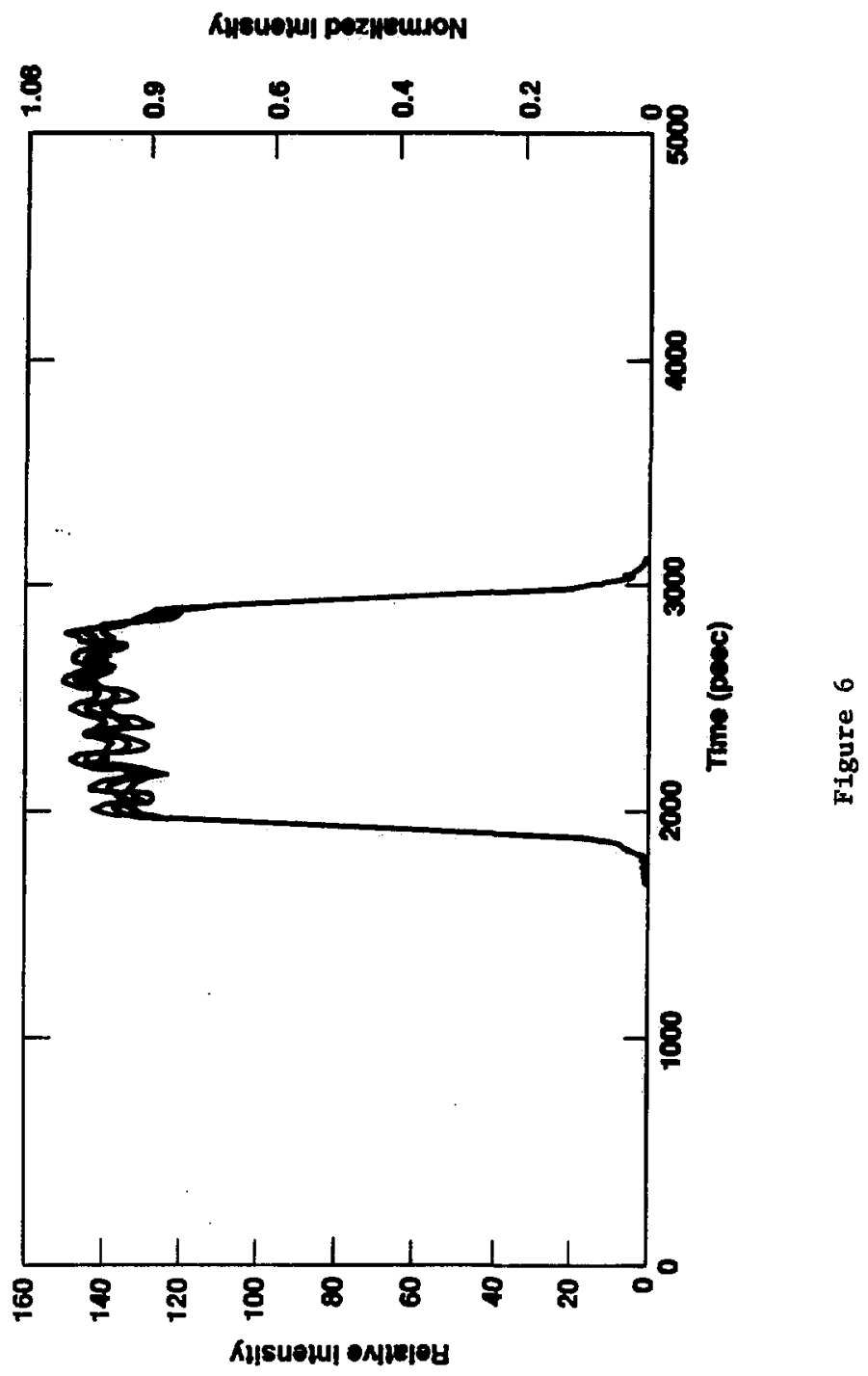


switched out oscillator pulses (100 ps FWHM) and the probe pulse (20 ps FWHM). Calibrated calorimeters were used to measure the beam energy. Both scattered and transmitted beam energies off of the target were measure for each shot using both external calorimeters and an array of photodiodes mounted inside the target chamber. Calibration target shots were conducted before, during, and after the series of experimental target shots to ensure precise energy-on-target (EOT) measurements. This is an important laser parameter to know for running laser-plasma hydrodynamic compuier simulations. The spatial energy distribution of the laser focal spot has been characterized by the KMS staff and estimated to be approximately $\pm 20 \%$. $\mathrm{X}$-ray pinhole cameras were fielded as both plasma and laser diagnostics. The uniformity of the $x$-ray pinhole images (approximately $\pm 10 \%$ ) indicate that the energy of the laser beam was fairly uniformly distributed over the laser focal spot and consistent with the $\pm 20 \%$ spatial laser energy distribution measurements. The size of the pinhole images also confirms the laser irradiation area (laser focal spotsize). The CHROMA laser was able to deliver consecutive target shots with energy fluctuations of $10 \%$ or less. This allowed for a rapid compilation of high quality data which permitted the detailed study of these plasmas over a wide range of parameter space.

\subsection{Simultaneously Space and Time Resolved Diagnostics}

Important components of these experiments were the simultaneously space and time resolved instruments that were employed together for the first time to study the laser-produced plasmas. These instruments were aligned to view the target edge on (within $5^{\circ}$ of the target surface). The basic experimental set-up is shown in Figure

7. Spatial and temporal gradients were resolved using 3 new diagnostics simultaneously: a 4-frame holographic interferometer, a modified x-ray streaked 
Figure 7. Schematic diagram of the experimental set-up in the CHROMA target chamber. The main plasma diagnostics are aligned ( 5 approximately $5^{\circ}$ ) in the plane of the target, perpendicular to the expanding plasma plume. These important new diagnostics include the multi-frame holographic interferometer, the Framing Crystal X-ray Spectrometer (FCXS), the X-ray Streaked Crystal Spectrograph (XSCS), and a spatially resolved, time integrated crystal spectrograph. 


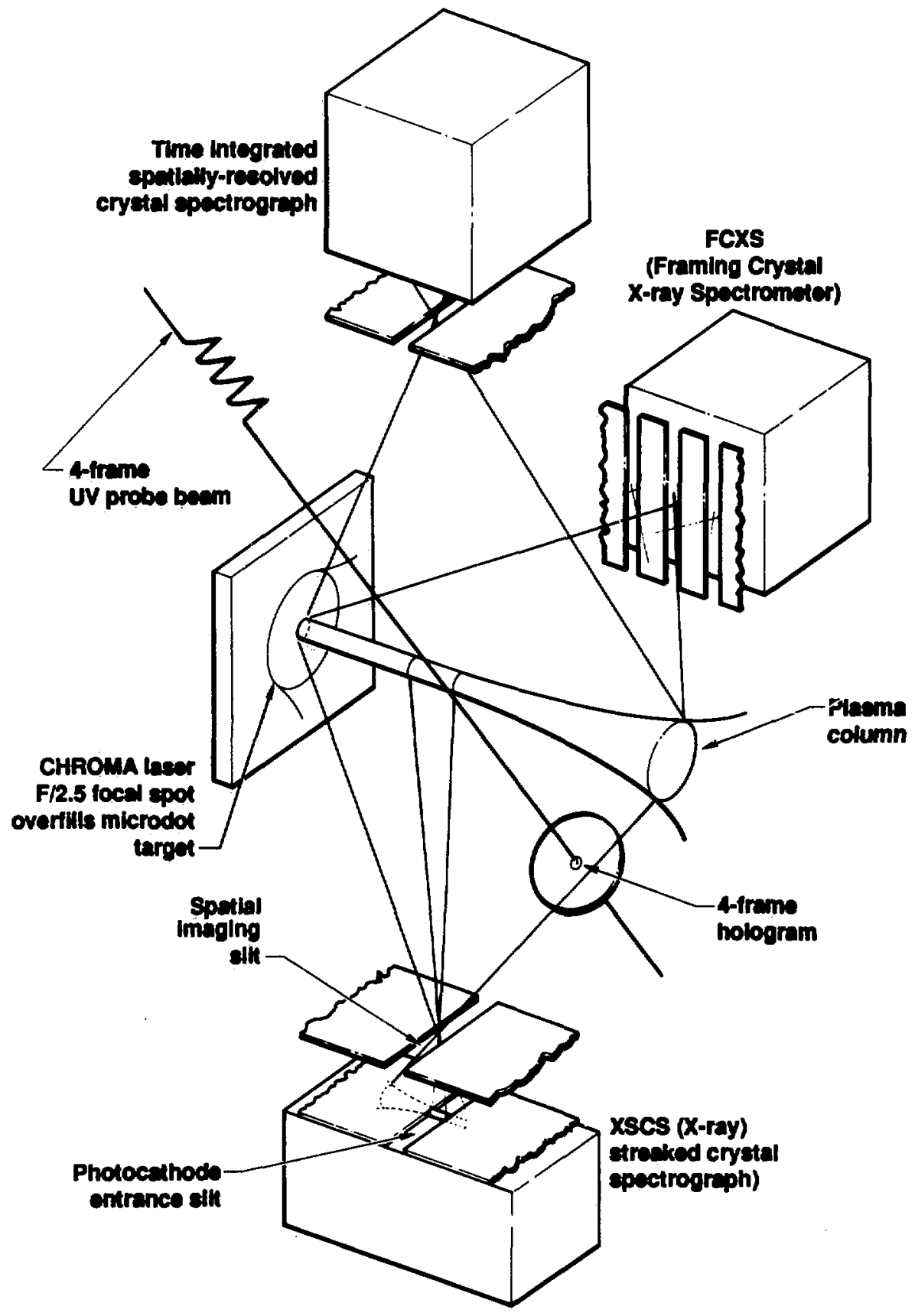


crystal spectrometer with coarse spatial imaging (XSCS), and a framing crystal x-ray spectrograph (FCXS). Conventional time-integrated, spatially resolved crystal spectrometers and pinhole cameras were also fielded to complete the experimental set-up. These instruments are described in detail below.

\subsubsection{Framing Crystal X-ray Spectrometer (FCXS)}

The Framing Crystal X-ray Spectrometer (FCXS) measured simultaneously space and time resolved X-ray spectra. A photograph and schematic of the FCXS are presented in Figure 8. The development and use of this instrument was key to the success of these experiments in minimizing spatial and temporal gradients which have compromised the interpretation of spectral data measured in previous laser-plasma experiments. [Boiko et al. (1984), Kauffman et ạl. (1985; 1987), Lee et al. (1982), Bailey et al. (1986); Gauthier et al. (1983; 1985; 1986; 1987)] The active element of the FCXS was a directly-gated, proximity focussed microchannel plate (MCP) framing camera. Similar time resolved spectrometers based on early versions of simpie MCP detectors have been built and fielded to study other high energy-density plasmas with approximately $5 \mathrm{~ns}$ time resolution. [Nahrath et al. (1977), Fehl et al. (1980), Helava et al. (1979)] In these detectors, the time frames were generated by gating the anodes of the detectors. This technique was used by Stewart (1983) to study $x$-rays produced during z-pinch pulsed-power experiments with a 1-2 ns time resolution. Similarly, this method was used by Marrs et al. (1983) to measure time resolved spectra from z-pinch pulsed-power experiments using a vacuum-ultraviolet grazing incidence spectrometer. Directly-gated MCP detectors were suggested by Stewart (1983) in which transmission striplines would be coated onto the MCP and gated on and off to attain higher time resolution and improved dynamic range. Directly-gated MCP spectrometers have been successfully 
Figure 8. A photograph and schematic diagram of the Framing Crystal X-ray Spectrometer (FCXS) which provided the bulk of the simultaneously space and time resolved $x$-ray spectra used in this work. The FCXS uses a flat Bragg diffraction crystal to measure x-rays emitted from the laser-plasmas. A gated microchannel plate (MCP) detector (instead of the usual $x$-ray film) is used as an active readout and for signal amplification. This allows the FCXS to record 3 separate, variably spaced, $\geq 100$ ps long, time frames. A set of 3 slits are used to spatially image the laser-plasma onto each MCP stripline. 

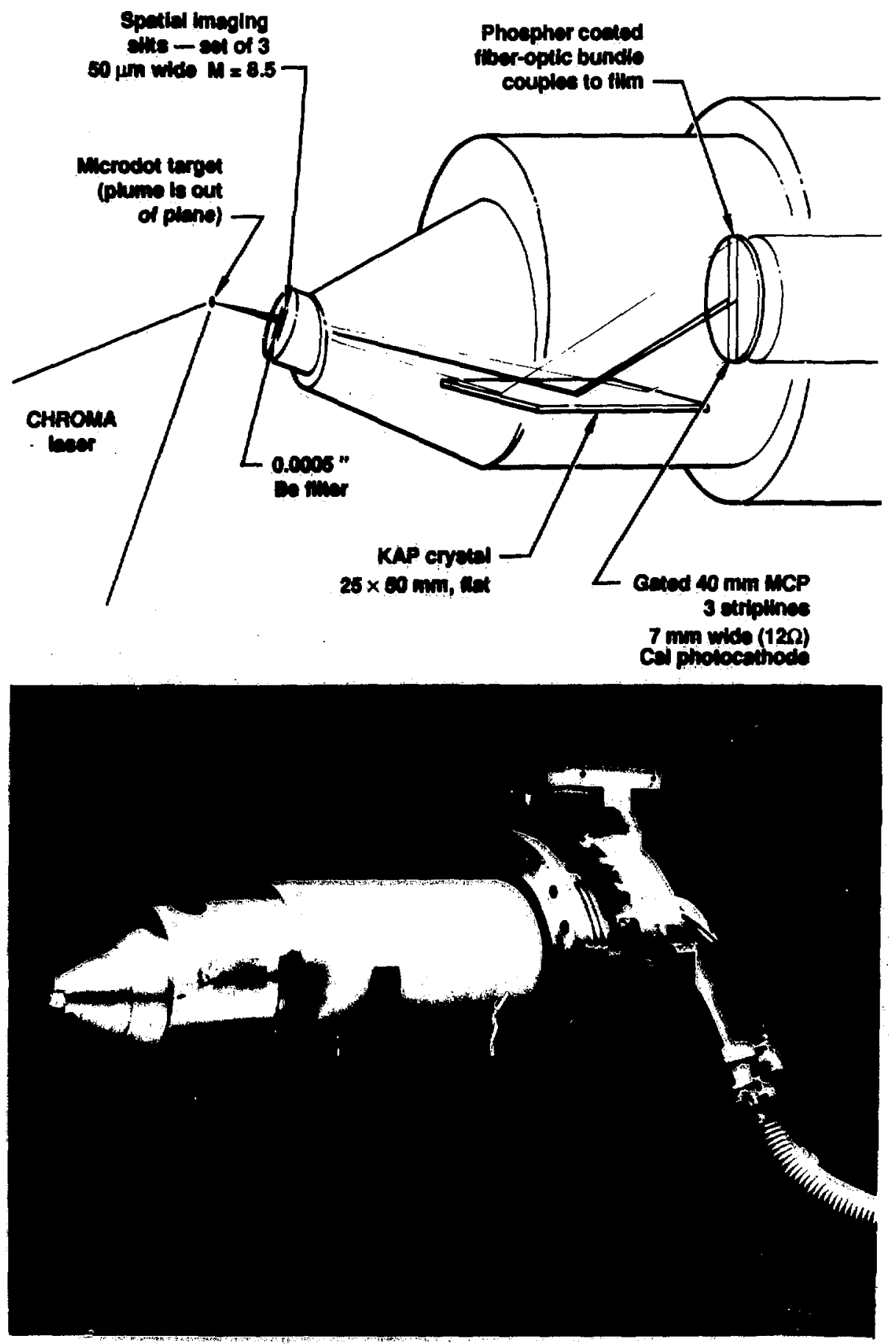
used to study high energy plasmas produced by z-pinch pulsed-power produced plasmas with 1-5 ns time resolution. [Price (1983; 1988)] Eckart et al. (1985) and Matthews et al. (1985) used a directly-gated (gated off only) MCP to study vacuum-ultraviolet emission from laser-produced plasmas with $\approx 1$ ns time resolution. A directly-gated (gated on then off) MCP spectrograph was first used to measure the simultaneously space and time resolved x-ray emission from a laser-produced plasma. [Young et al. (1986)] In the present work, these directly-gated MCP framing instruments are used to conduct a detailed study of high-powered laser-produced plasmas and the physics of high-z ions.

Several improvements were made to the framing $x$-ray spectrometer to enable it to be used to study laser-produced plasmas. The directly-gated MCP framing camera used in the FCXS had a rise time of less than 50 ps and time frames as short as 100 ps were achieved. The FCXS also required higher image magnification and higher signal intensification than the detectors used previously. This is mainly due to the small plasma volumes and short time scales characteristic of laser-produced plasmas. A brief description of the gated MCP detector follows.

\subsection{1a. The Gated Microchannel Plate Framing Camera}

The gated MCP camera is based on a $40 \mathrm{~mm}$ diameter MCP as the main amplifying component in a proximity focused imaging detector. The gated MCP framing camera is shown in Figure 9. The MCP operates as a large array of $10^{4}$ to $10^{7}$ miniature electron multiplier tubes aligned parallel to one another. The MCP is directly sensitive to both charged particles and energetic photons between 1 and $1500 \AA$. Both $\mathrm{x}$-ray and ultraviolet photocathodes can be used to extend the sensitivity of the MCPs. [Fraser et al. (1984)] The MCP provides high signal amplification (gains of $10^{2}$ to $10^{4}$ ) with high spatial spatial resolution $(\leq 50 \mu \mathrm{m})$. The MCP had a active 
Figure 9. A schematic diagram of the gated microchannel plate (MCP) framing camera. X-rays incident on the front MCP surface create photoelectrons which are accelerated through the multiple channels of the MCP via a gated bias voltage. The MCP acts as a high gain, electron multiplier converting the detected $x$-ray(s) into a burst of secondary electrons which is then accelerated onto a phosphor coated fiber optics bundle. The phosphor converts the signal into visibl- ight which can be recorded using fast visible film outside of vacuum. By gating the MCP bias voltage on then off, the gated MCP framing camera can provide time resolution of under 100 ps and a spatial resolution of approximately $50 \mu \mathrm{m}$. 


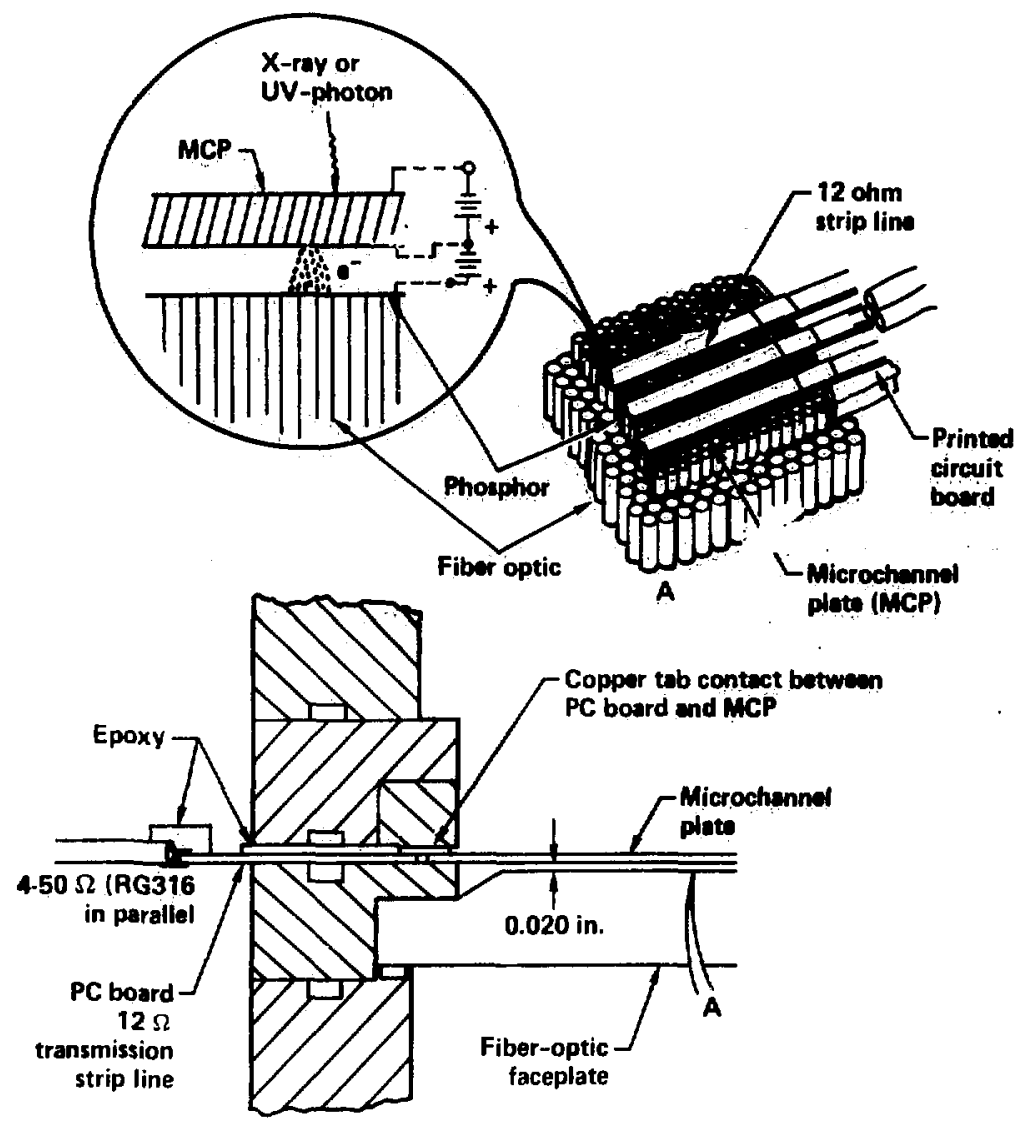

Figure 9 
area of $1.26 \times 10^{3} \mathrm{~mm}^{2}$ with $12 \mu \mathrm{m}$ diameter channels, $15 \mu \mathrm{m}$ center-to-center spacing $0.508 \mathrm{~mm}$ thick, $5.5^{\circ}$ bias angle, and an length-to-diameter aspect ratio of 42. Uncouled 40 mmdiumeter MCPs were obtained from Varian Corp.

A $2000 \mathrm{~A}$ thick gold ground plane was vapor deposited onto the backside of the MCP at an angle of $45^{\circ}$. The MCP was slowly rotated during the plating process to ensure a uniform conting inside the channels and on the webbed cladding area between the microchannels. This provided a high conductence ground plane with good end focusing preperties. This is important to optimize the spatial resolution in the proximity focuselweotifiguration used in these detectors. A set of throe parallel striplines were comedtonto the front surface, perpendicular to the microchannel bias ande of the MCP channels. The striplines were made with $2000 \AA$ of gold conted at approximately $15^{\circ}$ dopesition angle to the MCP surfece. The botal DC resistance

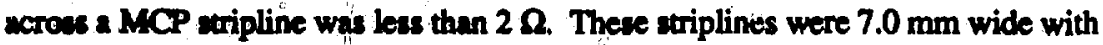
29.0 man cencr-to-center spacing. The MCP striplines formed a $12.5 \Omega$ impedance micromip trusimiscion line. The stripline impedance was determined using a semi-empirical formula of Dworky (1979) for a parallel plate (infinite ground plane) microwipline and was verified using time-domain-reflectometry (TDR). The same TDR mohod was ued tooptimize the specing between the MCP striplines to minimize high frequency cross talk between the striplines.

The bare MCP'slas has a quantum efficiency of approximately 2-5\% for 1 to 4 keV $r$-rays (for near normal incident angles). This quantum efficiency is increased by a factor of 3 to 5 for a CsI conted MCP. [Fraser et al.(1984); Wiza (1979)] The front wurface of the MCPs used in these devectors were overconted with a $5000 \mathrm{~A}$ thick Cal photocathode which converted the detected $x$-rays into electrons. [Fraser et al.(1984); Wiza \{1979); Henke et al. (1981; 1982); Premaratre et al. (1983); Saloman et al. (1980)] The MCP's are made of a high lead content glass which has been activated by a high temperature firing in a hydrogen atmosphere. This prosess 
increases the secondary electron yield of the MCP glass. Electrons produced by the $x$-ray photocathode were accelerated through the microchannels of the MCP creating a cascade of secondary electrons. Each detected photon was converted into approximately $10^{2}$ to $10^{4}$ secondary electrons.

After exiting the MCP, the electrons were accelerated across a small gap (0.508 $\mathrm{mm},+2.5 \mathrm{kV}$ ) onto $\mathrm{P}-11$ phosphor coated fiber optic faceplate converting approximately $5 \%$ of their energy into forward directed visible light peaked at a wavelength of $526.0 \mathrm{~nm}$. The fiber optic faceplate [Galileo Electro-Optics Corp.] was conted with a thin coating of $\mathrm{InSnO}_{2}$ to ( $500 \Omega /$ square) which acted as an anode. Approximately $1.5 \mathrm{mg} / \mathrm{cm}^{2}$ of $\mathrm{P}-11$ phosphor [Lumilux Blue B11-02 from Riedel-De Heen AG Seelze-Hannover] was gravity settled onto the fiber optic surface. A small amount of neopreme rubber cement was used to help bond the phosphor particles. Toluene was used as the solvent. The electrons were converted into a light pulse which was transmitted through the fiber optic faceplate out of vacuum, then through another fiber optic bundle $[40 \mathrm{~mm}$ diameter $\times 38.1 \mathrm{~cm}$ long, N.A.= .66, from INCOM Corp.] to an accessible location (outside the target chamber) where the signal was recorded using Kodak Royal X-pan film. An ITT 4113 image intensifier tube was used between the last fiber optic bundle and the film plane to provide additional signal intensification. Fiber optic components were butt mounted to each other using optical coupling grease in all interfaces. The spatial resolution of this type of MCP based proximity focussed detector has been measured to be approximately $50 \mu \mathrm{m}$ with a dynamic range of approximately 100 at peak gains by Stewart (1983).

Electrical centact was made to the MCP striplines via a printed circuit board. [Synthane-Taylor type G10H-white] A thin cout of epoxy on the PC-board was used to provide a vacuum o-ring sealing surface. The PC-board had a pattern of three $12.5 \Omega$ parallel striplines with a center-to-center spacing of $9 \mathrm{~mm}$ to match the 
MCP stripline pattern. Each MCP stripline was driven by four parallel $50 \Omega$ semi-rigid coaxial cables (UT-50) with SMA connectors. The MCP was operated at -700 to -1200 volts and the anode run at +2500 vdc. All electrical connections were impedance matched to within a few percent to minimize pulse distortion and attenuation. These were tested using time-domain-reflectometry on a Tektronix 7S12 sampling oscilloscope. The three MCP striplines were matched to better than 5 ps in length to insure accurate timings between striplines. The electrical rise time across each striplines was less than $50 \mathrm{ps.} \mathrm{The} \mathrm{high} \mathrm{frequency} \mathrm{cross-talk} \mathrm{between}$ adjecent striplines was measured to be less than a few percent. A more complete description of similar MCP framing cameras can be found in Stewart (1983) and Young et al. (1986).

The conted MCP striplines were part of a $12.5 \Omega$ transmission line which could be gated on and off with a very short bias voltage pulse across the MCP. By gating ..t. each stripline on and off at selected times, the FCXS recorded framed spectral data. Electrical high voltage puleers using $\mathrm{KN}-6 \mathrm{~B}$ Krytron pulsers, EIMAC Y690 blocking oecillator tubes, or avalanche transistor chains can be used for abnanowecond gating pulses. [Booth (1987); Benzel et al. (1985)] These high voluge pulsers can also drive a transmission line or bloom line with pulse shapening diodes to generate few hundred picosocond wide gating pulses with approximately 100 ps rise and falls times. [Booth (1987); Grekhov et al. (1981)] The time-jiber of these eloctrical pulsers is typically 100 ps or larger and is a major drawback in applying them to study very short-lived, highly transient laser-produced plasmas. The rise and fall times must also be accounted for in detarmining the temporal resolution of the MCP detector as well as the electron transit and spread times through the MCP. The transit time is the time duration for the electrons generaved from a single detected photon to traverse the microchannel and exit out the back of the MCP. The spread time is the spread in electron transit times (created 
from a single detected photon) of the electron shower exiting from the microchannel. For instance, the gating bias voltage pulse applied to the MCP stripline must be longer than the electron transit time. The MCP electron transit time has been measured to be $150 \pm 35$ ps at 900 volts and rise and fall times of approximately 50 ps. [Young et al. (1986)] This agrees with calculations of Ito et al. (1984), Oba, and Yamazaki et al. (1985) and indicate directly-gated MCP frame times of less than 100 ps are very feasible. [Also, see Kilkenny et al. (1988).]

\subsection{1b. The Auston Switch Tranamiation Line Puler}

For these experiments, an intrinsic silicon Auston switch transmission line pulser was used to generate gating pulses as short as 100 ps with rise and fall times of less than 50 ps. [Auston (1975); Koo et al. (1984); Christie et al. (1985)] The frame time used in these experiments was 250 ps. Silicon Auston switches were built into a transmission line pulse generating circuit. [See Figure 10.] These intrinsic silicon Auston switches were manufactured by J. Koo and R. Wilcox of Lawrence Livermore National Laboratory. The switches were made of gold doped silicon chips $3.0 \times 3.0 \mathrm{~mm}$ and approximutely $0.5 \mathrm{~mm}$ thick. These represent $25 \Omega$ impedance structures. Gold electrodes were plated onto the chips to provide electrical contact. Two $25 \Omega$ pulse generating striplines were used in parallel to drive each $12.5 \Omega \mathrm{MCP}$ stripline. Four $50 \Omega$ cables in were used in parallel to relay the high voltage pulse between the Auston switch pulser and the MCP framing camera. A short stripline was pulse charged to twice the desired output voltage through an isolating RC circuit. The charging pulse had a rise time of $300 \mathrm{~ns}$ and a flat top width of $2.5 \mu \mathrm{sec}$. [Christie (1984)] The charging palse was monitored on an oscilloscope as shown in Figure 11(top). The charging pulse shows a small discontinuity when the Auston switch becomes conductive due to a small but 
Figure 10. A photograph of the Auston switch pulser and charging circuit. An intrinsic silicon Auston switch is built into a $25 \Omega$ transmission line circuit. A short stripline is pulse charged to twice the desired output voltage through an isolating RC circuit. The switch becomes conductive when illuminated by the $20 \mathrm{ps}, \lambda=1.05 \mu \mathrm{m}$ laser pulse and quickly discharges the charge line voltage. The outpus pulse is approximately half the amplitude of the charge line voltuge and twice the length of the charged stripline. The Auston switch pulser is coupled to the FCXS detector $\because \quad=-$ using two $50 \Omega$ cables (type RG-316 with SMA connectors) in parallel. 


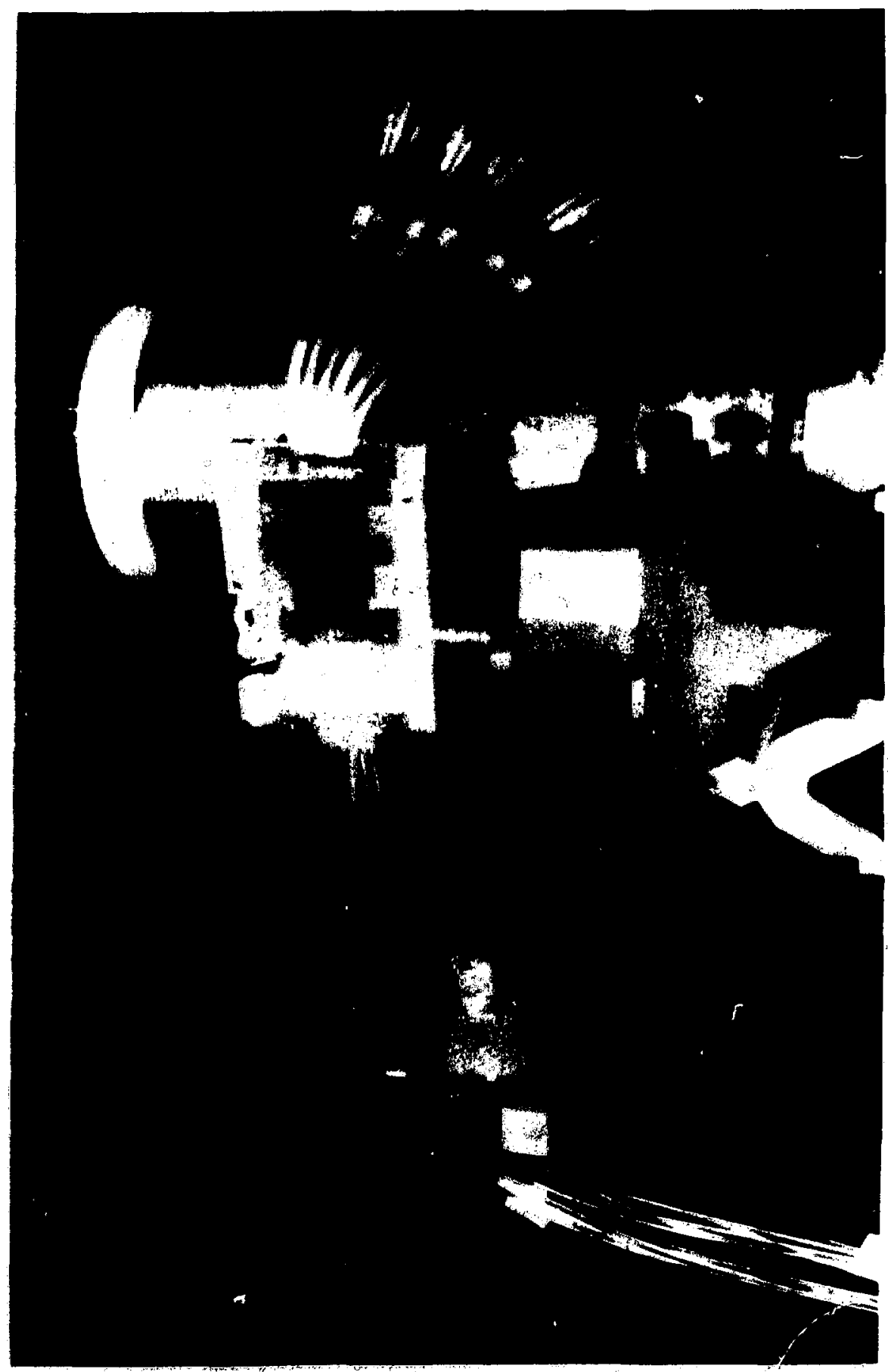

욱 
Figure 11. Typical (Tektronix 7104) oscilloscope traces showing Auston switch pulses after passing through the FCXS and 20 ns of cable show (top) a 2800 volt, $2.5 \mu \mathrm{s}$ wide voltage pulse used to pulse charge the Auston switch stripline; the fast rising signal indicates activation of the Auston switch, (middle) a normal output pulse, (bottom) a normal output pulse on $1 \mathrm{~ns} / \mathrm{div}$ time scale (approximately 1000 volts, 400 ps wide, and $\approx 50$ ps rise and fall times; limited by the $>300$ ps scope resolution). 
$-47-$

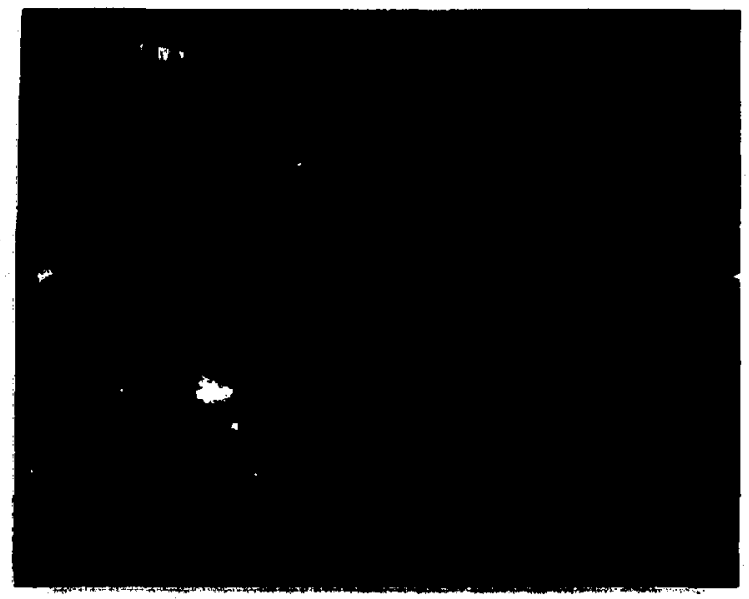

And

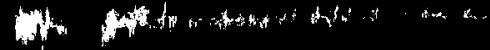

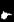

Figure 11 
measurable drop in voltage due to the recharging of the Auston switch charge line. This provided a reliable measurement of the relative timing of the electrical charging pulse and the optical Auston switch triggering pulse. The silicon switch was illuminated with the $20 \mathrm{ps}$ wide $(1.05 \mu \mathrm{m})$ holographic probe pulse before it was converted to $4 \omega_{0}$. The switch becomes conductive and discharges the charge line generating a square electrical pulse traveling along the transmission line in each direction away from the switch. The pulse traveling down the charge line encounters an open circuit with infinite impedance at the end of the charge line and reflects back down the transmission line. A square outgoing pulse travels down the load line until the reflected charge line pulse cancels it out at the Auston switch. The output pulse width is equal to twice the length of the charge line and its amplitude is approximately one half of the charge line voltage with some losses due to small reflection losses at the various electrical interfaces.

It takes approximately $100 \mu \mathrm{J}$ of $1.05 \mu \mathrm{m}$ light to activate each silicon switch. The electrical rise time of the switch is determined by the laser pulse rise time and also the rise time of the stripline. For these experiments, the laser switching pulse was taken from part of the $20 \mathrm{ps}$ pulse width holographic probe beam before it was frequency quadrupled and amplified (20-40X) using a Nd-YAG rod amplifier. The $1.05 \mu \mathrm{m}$ switching pulse was passed through an appropriate optical delay and focused using a cylindrical lens onto en array of six silicon switches. By using the laser probe beam to trigger the gating puleer, the relative timing of the FCXS was precisely known with respect to the holographic probe beam, the $x$-ray streaked camera, and the CHROMA laser heating pulse. All of the switches were activated simultaneously and therefore there is no timing jitter other than differences in the delay cables and striplines. The interframe time was adjusted by staggering the cable lengths (formed by using various combinations of SMA cables and connectors) between the Auston switch pulser and the MCP striplines. Since each stripline was driven by four $50 \Omega$ 
cables in parallel, the delay cables had to be tested and matched to better than 5 ps to minimize pulse distortion. All cables and connectors were tested via TDR in the laboratory before being used.

The output pulse from a similar Auston switch pulser has been studied in detail using a very fast Thomson CSF-TSN660 oscilloscope with a rise time of approximately 150 ps. This work was presented in Young et al. (1986). The very fast rise time of the Auston switch generated pulse is scope limited and must be estimated from the convolution of the probe beam activating laser pulse width (approximately $20 \mathrm{ps)}$ ) and the pulse rise time traversing the pulser, MCP detector

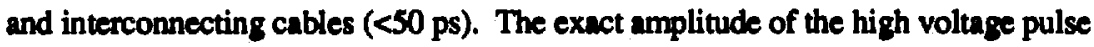
is also distorted by the scope rise time limitations. Again, this must be estimated from the charge-line voltage used and from the attenuation due to cable lengths (determined by TDR and sampling scope measurements).

The Auston switch output was successfully monitored throughout the experiments using a Tektronix 7104 oscilloscope with 7A29 amplifier. Fast microwave SMA cables and connectors were used as well as high voltage attenuators from Burth Electronics. A typical output pulse is presented in Figures 11(middle) and 11(bottom). This high voltage pulse is measured after traversing the MCP stripline and approximately $20 \mathrm{~ns}$ of connecting cable. The shape and amplitude of the pulse was oscilloscope limited (approximately a 300 ps rise time). This was not a significant limitation for these experiments as these were not absolute measurements and only relative amplitudes from shot-to-shot monitoring of the MCP bias voltage pulse was required. The pulse shape was measured by low voltage tests in the laboratory using a fast sampling scope and the relative amplitude of the output pulse was inferred from the charge line voltage and by monitoring the shape of the switched out pulse.

The output pulse of each of the MCP striplines were monitored for every shot. 
This monitoring provided guidance in determining good FCXS data. For instance, in Figure 12(top), a poor ouput pulse is presented. In this case, the amplitude of the laser pulse illuminating the Auston switch was very low. This is indicated by the low amplitude, pulse shape (the slow falling back edge of the pulse), and increased pulse width of the scope trace. The low amplitude of the activating laser pulse did not create enough charge carrier pairs to make the Auston switch fully conductive. This left a residual resistance in the Auston switch which generated multiple reflections in the high voltage pulse between the partially conducting Auston switch and the open circuit in the charge line. Instead of a clean single output pulse, a lower amplitude pulse was transmitted through the switch followed by a family of reflections with decreasing amplitudes. Thus, the ouput pulse could be effectively (qualitatively) monitored using the Tektronix 7104 oscilloscope.

Additionally, the Auston switch pulser was very sensitive to prepulses in the infared probe beam illuminating the switch array. A series of prepulses were generated in the CHROMA oscillator cavity due to Q-switch leakage of the oscillator trin. The very short charge lines used in these experiments contained a finite amount of charge. The series of prepulses incident on the Auston switch would generate enough charge-carrier pairs to bleed off a significant fraction of the stored up charge and affect the amplitude of the output pulse. The probe beam, Auston switch activating pulse was constantly monitored for these prepulses. Prepulses were minimized to less than $10^{-3}$ of the probe beam pulse (approximately $0.1 \mathrm{~mJ}$ at the switch). Again, the prepulse problem was monitored by measuring the Auston switch pulse with the Tektronix 7104 oscilloscope. A oscilloscope trace of a prepulse affected output pulse is presented in Figure 12(bottom). The prepulse is indicated by the "toe" (long, slow rising front edge of the pulse) and the low amplitude of the switched out pulse. The "toe" consists of a series of prepulses resembling small ramps with a period of $8 \mathrm{~ns}$; exactly the CHROMA oscillator cavity 
Figure 12. Scope traces of poor Auston switch activation due to (top) low laser energy on Auston switch causing multiple reflections in the transmission line circuit, and (bottom) a laser prepulse problem in licated by the "toe" and low amplitude of the pulse. 

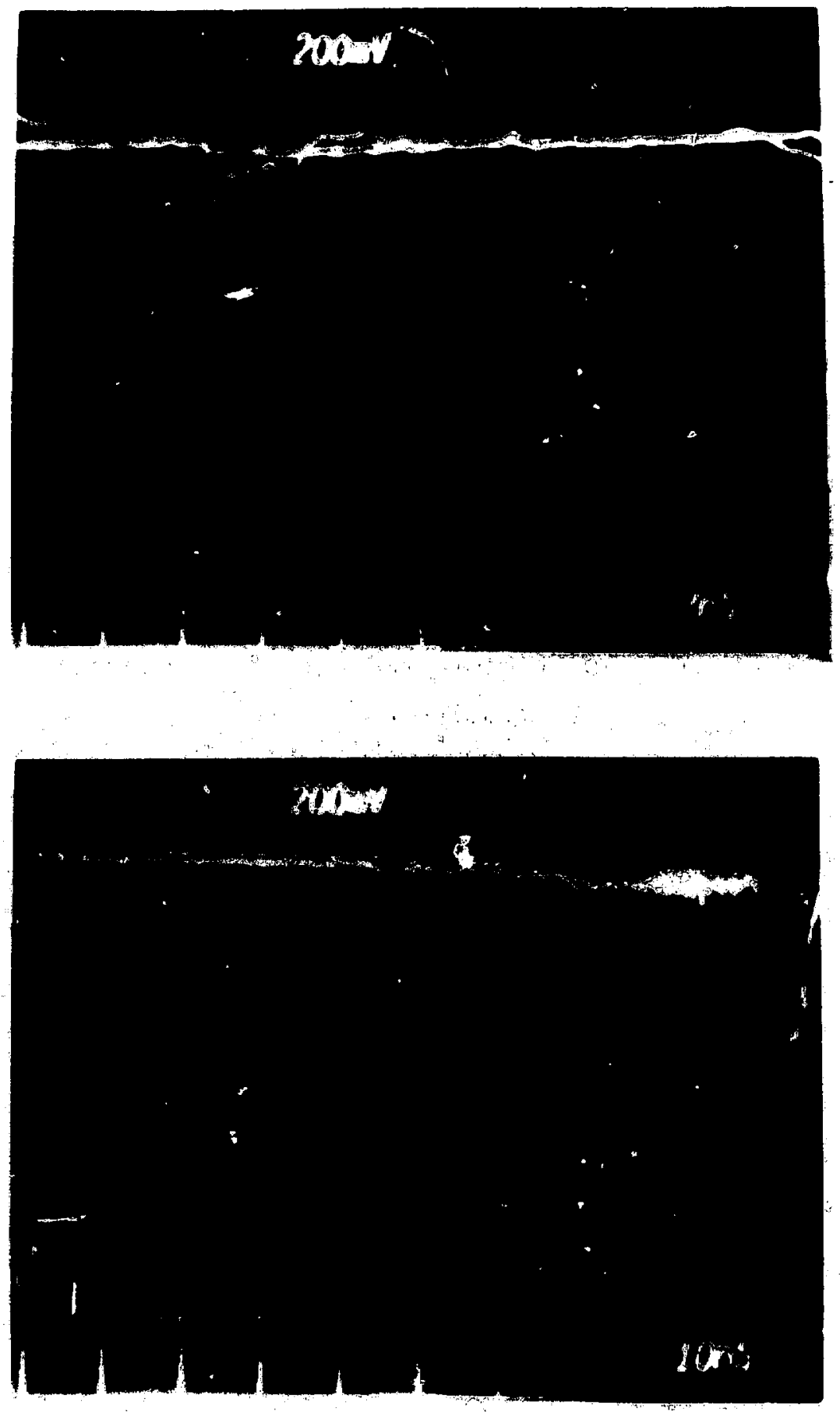

Figure 12 
length. The prepulse problem was reduced by tuning the CHROMA oscillator cavity Q-switches, by using polarizers in the regenerative amplifier cavity, and by using a $10 \%$ low level transmitting dye cell.

\subsection{1c. Other Important Features of the FCXS}

The FCXS used the gated MCP framing camera as the active detector of a conventional spatially imaged flat crystal spectrometer. A set of three parallel slits located $1.8 \mathrm{~cm}$ from the target was used to spatially image the $x$-ray emitting plasma plume independently onto each MCP stripline. Time resolution was achieved by gating each stripline on and off at different times. The result was that three high magnification spatially resolved $x$-ray spectra were measured, each one representing a different time interval. Approximately $800 \mu \mathrm{m}$ of the plasma plume (spectral image) including the target surface was imaged onto each stripline. Alternatively, the striplines were run with different MCP bias voltages to increase the dynamic range of the instrument or with different $x$-ray diffraction crystals to incresse the spectral coverage of the spectrometer. Typical FCXS framed spectra are shown in Figures 13 (Shot \#843), 52 (Shot *8502), and 54 (Shot \#8519) for Al, Mo/Mg, and Ag microdot target laser plasmas, respectively. Details of these messurements are described in Chapters 5 and 6. For the Al microdot experiments, a $2.5 \times 6.0 \mathrm{~cm}$ flat KAP crystal was used to measure x-ray emission over a wayelength range of 4 to 8 $A$ with a resolution, ( $(N \Delta \lambda)$, of 700 to 1000 depending on the wavelength of the detected x-ray. For the Ag microdot experiments, a $2.5 \times 5.9 \mathrm{~cm}$ flat ADP crystal was used to measure $x$-ray emission over a wavelength range of 2.7 to $4.4 A$ with a resolution of over 1000. For the Mo/Mg microdot experiments, two crystal were used to measure the Mo L-abell and Mg K-shell spectra independently. A $2.5 \times 6.0$ cm flat KAP crystal was used to measure Mg K-shell x-ray emission over a 
Figure 13. FCXS data for Shot $\# 8433(100 \mu \mathrm{m}$ diameter aluminum target shot at $1.0 \times 10^{14} \mathrm{~W} / \mathrm{cm}^{2}, \lambda=0.53 \mu \mathrm{m}, \approx 1000$ ps stacked pulse width) showing the spatially resolved aluminum $\mathrm{K}$-shell radiation emitted during the time intervals $730-980$ ps (middle), 1230-1480 ps (top), and 1730-1980 ps (bottom). The x-ray emission is seen extending from the target surface (top of each stripline) out towards the incident heating laser pulse with a $50 \mu \mathrm{m}$ spatial resolution. 
$+1220-1490$ ps

1114

+730-100 po

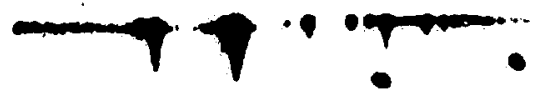

$+1730-1200$ pe

(cpproximently

13X gein)

11 lini:

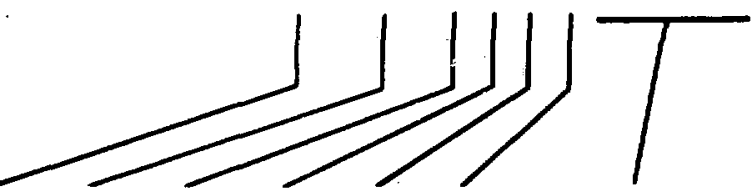

$H(2 p) H(2 p) H(3 p) H(4 p) H(3 p) H(4 p) H-H k e$ b-continuum

Figure 13 
wavelength range of 5.5 to $8.5 \mathrm{~A}$ with a resolution of 700 to 1000 depending on the wavelength of the detected X-ray. A $2.5 \times 5.0 \mathrm{~cm}$ Beryl crystal was used to measure the Mo L-shell spectra over a wavelength range of 3.7 to $4.4 \AA$ with a resolution of approximately 1000 . The FCXS had a magnification of 8.5. The imaging slits were $50 \mu \mathrm{m}$ wide ( $-55 \mu \mathrm{m}$ spatial resolution) and the MCP located $19 \mathrm{~cm}$ from the target. [ $\Delta x=d \cdot\left(1+M^{-1}\right)$ ] For this magnification, the spatial imaging slits were 1.0 mm apart in a $100 \mu \mathrm{m}$ thick tungsten substrate. A $12.5 \mu \mathrm{m}$ thick Be filter was used to protect the spatial imaging slits from turget debris and to filter scattered visible and ultraviolet light from the detector.

The FCXS was fielded simultaneously with a wide array of other diagnostics and had to be streamlined in a reentmant design to get as close as possible to the plasma source to maximize the measured signal (subtended solid angle) and spectral coverage without interfering or obscuring the field of view of the other diagnostics. Also, the spectrometer was shielded from any scattered laser light using a light-tight housing. Degradation of the highly hygroscopic CsI photocathode and MCP was minimized to maintain the shot-to-shot reproducibility of the instrument by keeping the MCP detector under continuous vacuum. An isolation valve was used to keep the MCP franing camera under continuous oil-free vacuum of $5 \times 10^{-5}$ torr or better. [See Figure 14.] The valve was opened before each shot only when the target chamber vacuum was $5 \times 10^{-5}$ torr or better and closed immediately after the shot. The isolation valve utilized a flapper valve design and a screw action to open and ciose it.

The FCXS spectral data was digitized using a Perkin-Elmer PDS scanning densitometer. A $25 \times 25 \mu \mathrm{m}$ scanning slit was used with $25 \mu \mathrm{m}$ increments. Typically, a 7 × $35 \mathrm{~mm}$ area of film was digitized for each MCP stripline creatino very large data files ( $280 \times 1400$ data points). The instrument response was removed (data converted into intensity versus wavelength and image distance) in the 
Figure 14. Photographs of the FCXS showing key features of the instrument including (top) the flat diffraction crystal inside of tie nosecone and (bottom) the isolation flapper valve used to keep the MCP detector under continuous high vacuum. 


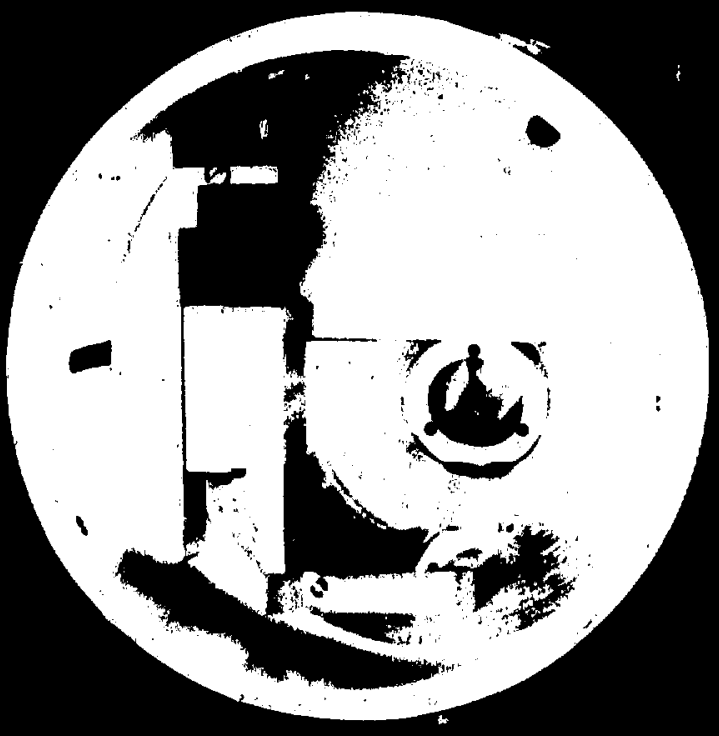

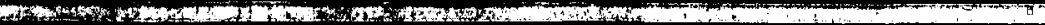

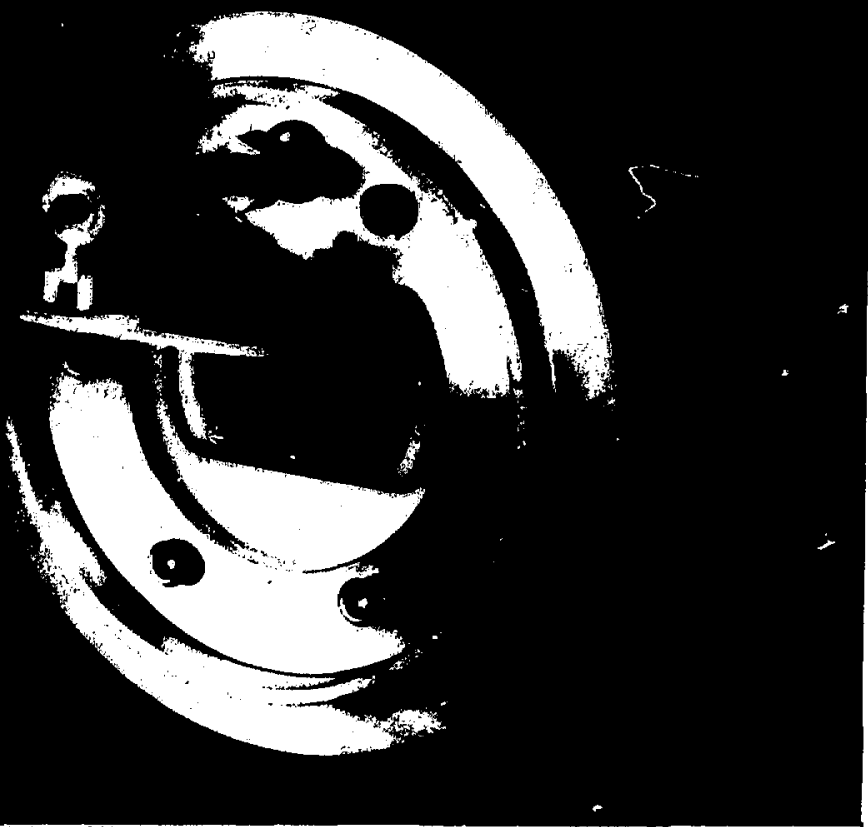


large three dimensional data arrays before any averaging was done. The experimental wavelength scales were determined by comparing the characteristic $\mathrm{K}$-shell or L-shell resonance lines with the theoretical wavelengths of Scofield's relativistic multiconfigurational Dirac-Fock "RAC" code and fitting the data to the flat Bragg crystal $x$-ray diffraction (dispersion) equation. $\left[m \lambda=2 d \cdot \sin \theta_{B}\right.$, where $m$ is the order number, $\theta_{B}$ is the Bragg angle, and $2 d$ is the crystal $2 d$ spacing in angstroms from Burek (1976).] The flat crystal dispersion is nicely formulated by a number of authors including Criss (1979) and Koppel (1977). Data analysis was done using "SOCKITTOME", a large data processing code run on the CDC-7600 mainframe computer at LLNL. [H. Finn (1976)] Each spatial increment was an average of 16 scans ( $400 \mu \mathrm{m}$ of film) and represented approximately a $47 \mu \mathrm{m}$ region of plasma. Each piece of film was preexposed using a calibrated step wedge optimized for a $P-11$ phosphor $\left(\lambda_{\text {peak }}=523.0 \mathrm{~nm}\right)$. The crystal reflectivities were accounted for using the calculated reflectivity curves of Burek (1976), Glinsky (1985), and Rockett et al. (1986). The filter transmissions and photocathode efficiencies were corrected for using the atomic data ubles of Henke et al. (1981; 1952). All of the spectroscopic instrument; were not abeolutely calibrated. Instead, the relative instrumental response has been accounted for in a "piece-meal" fashion. This may be a source of some uncertainty in the measured data. Therefore, in this work, I have atrempted to compare emission features (line intensities) that occur spectrally nearby each other to minimize uncertainties in the instrumental response. In future work, it is strongly recomended that the spectroscopic instruments be (abeolutely) calibrated as a integrated instrument. 


\subsubsection{X-ray Streaked Crystal Spectrograph (XSCS)}

$X$-ray streak cameras are commonly used as the detectors for simple $\mathrm{x}$-ray spectrometers to diagnose laser produced plasmas. [Key et al. (1980); Kilkenny et al.(1980); Kauffman et al. (1984); Matthews et al. (1984)] They are valuable diagnostics providing a continuous time record of the $x$-ray spectra. The instrument typically views and therefore spatially integrates over the entire plasma volume. Used in this way, the measured spectra samples a range of spatially averaged plasma conditions such as the electron temperature, $T_{e}$, the electron density, $n_{e}$, and the ion density, $n_{j}$. Thus, the interpretation of the data becomes suspect due to unresolved spatial gradients.

The X-ray Streaked Crystal Spectrometer (XSCS) used in these experiments was a modified KENTECH x-ray ureaked camera. [Charatis (1986; 1987)] The instrument incorporated a pair of slits to act as a collimator which restricted the field of view of the instrument. This is illustrated in Figure 15. The first slit located in front of the diffraction crystal about $1.5 \mathrm{~cm}$ from the plasma source imaged the $x$-ray emitting plasma plume onto the plane of the photocathode. The streak camera had a $800 \mu \mathrm{m}$ wide entrance slit located in front of the CsI photocathode to limit the flux into the streak camera electron imaging tube. This photocathode slit also transmitted a selected region of the plume image onto the photocathode and helped define the temporal resolution of the instrument. The XSCS was normally operated with a 65 $\mu \mathrm{m}$ wide imaging slit located $12 \mathrm{~cm}$ from the target. The magnification was about 7 .

The plasma region sampled by the XSCS was determined using a multi-step process. The photocathode slit was replaced by a $100 \mu \mathrm{m}$ diameter wire acting as a spatial fiducial. By removing the narrow $(800 \mu \mathrm{m})$ photocathode slit, the entrance opening into the streak camera was widened to a few millimeters. The wire was placed exactly on the center position of the photocathode slit. A time-integrated 
Figure 15. A schematic diagram of the two slit, spatial imaging employed by the XSCS instrument (bottom), The two slits act to collimate a selected $170 \mu \mathrm{m}$ region of plasma viewed by the streak camern entrance slit. The XSCS entrance slit could be opened to several millimeters to record a time integrated, spatially resolved spectra to locate the region of plasma viewed by the detector. A wire spatial fiducial was then placed precisely at the center of the entrance slit. The shadow of the wire fiducial cast onto the recorded image corresponds to the approximate location of the $170 \mu \mathrm{m}$ region of plasma viewed in the previous target shots (top). 

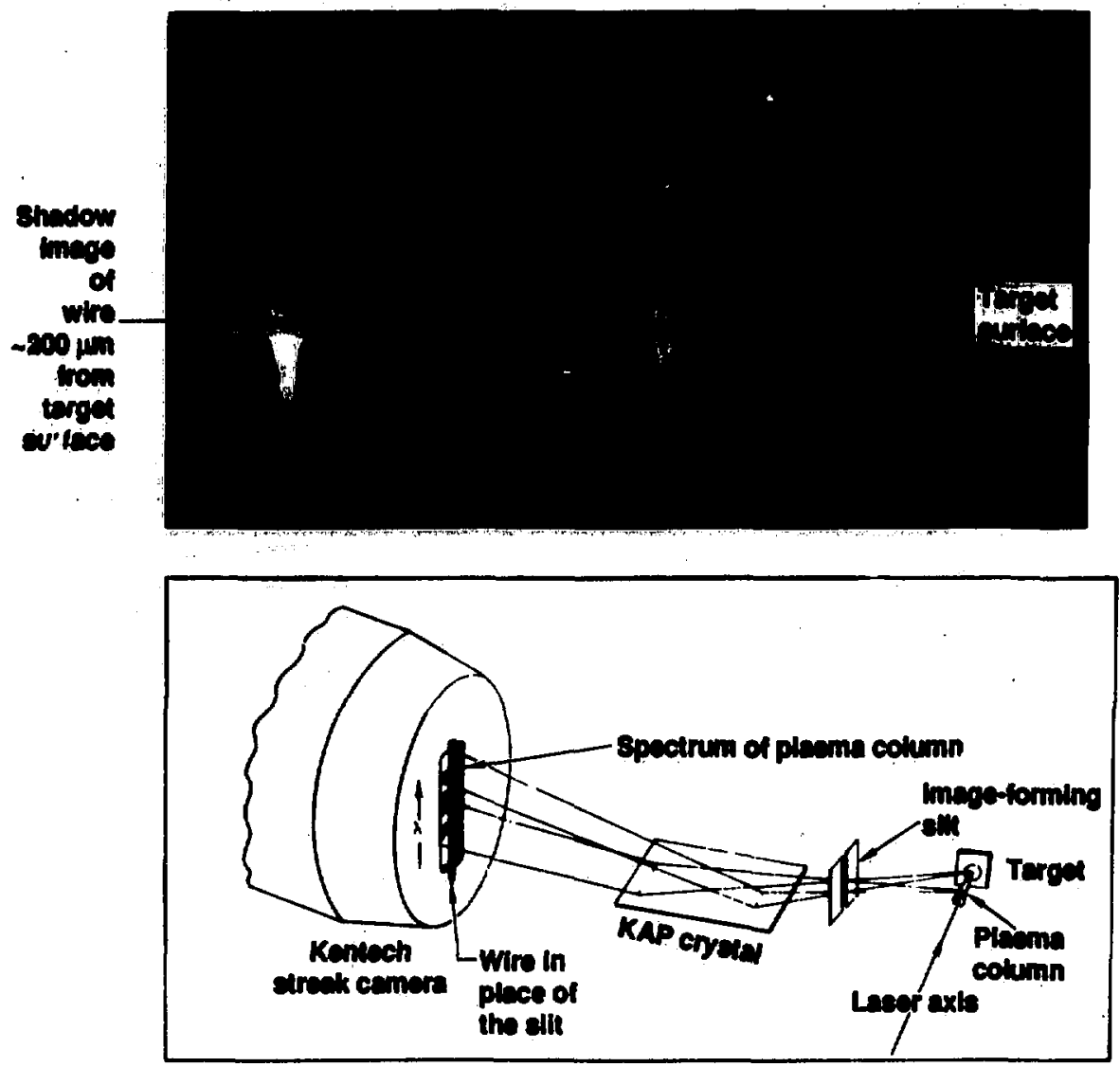

Salection of en x-rey cmitting volume of the plasma column for recording by the $x$-ray stredk emera 
spectrum was then measured during the next target shot with the wire spatial fiducial casting a shadow on the recorded data. The wire fiducial was then carefully replaced with the $800 \mu \mathrm{m}$ wide photocathode slit centered exactly on the wire position. The region of plasma sampled is therefore the column of plasma subtended by the width of the photocathode slit and centered on the middle of the shadow cast by the wire. This was approximately $170 \mu \mathrm{m}$ region of plasma for the magnification and the slit widths used in these experiments. The slits were adjustable in width and in location and various regions of plasma were sampled over a sequence of similar target shots. The strak camern was normally operated with a $60 \mathrm{p} / \mathrm{mm}$ sweep speed. This provides approximutely $30 \mathrm{ps}$ time resolution. A timing fiducial was aved for each shot generated by the uv-probe holographic interferometer probe pulse and coupled to the Cal photocathode using an Al conted silica fiber optic cable. The timing was measured to betver than 5 . ps with respect to the CHROMA luer heating pulse. The timing fiducial is essential to correctly interpret the streaked $x$-ray data and compare it with data reconded with other instruments. The various sweep speeds for the KENTECH detector were calibrated. A series of equally spaced ultraviolet laser pulses were generated using the CHROMA laser and its pulse stacking capability. This ultraviolet light was then scatter off a $400 \mu \mathrm{m}$ diameter steel target ball onto a thin aluminum photocathode. The scattered light was measured and the various sweep speeds determined from the time interval separating the individual ultraviolet light pulses. The timing fiducial was similarly verified by measuring the scattered light off of the steel target ball and accounting for the time of flight between the steel ball located at the target position and the position of the streak camera photocathode. The sweeping (deflection) field of the KENTECH streak camera was not totally uniform especially near the edges of the tube. This was measured by recording the time resolved (swept) $x$-ray emission of a point source viewed through a series of equally spaced collimating holes. This field distortion was determined for the entire 
streak camera temporal range by varying the timing separation between the onset of the $x$-ray source generated via the CHROMA laser and the KENTECH electron deflection trigger. The field distortion of the electron tube optics was characterized and removed from the data with convolution mapping software.

A flat $3.5 \mathrm{~cm}$ potassium acid KAP (potassium acid phthalate) diffraction crystal was used for the Al microdot shots providing a wavelength coverage of 5 to $8 \AA$ with a resolution of 500 depending on the wavelength of the detected $x$-ray. A flat ADP (ammonium dihydrogen phosphate) diffraction crystal was used to measure the L-shell Ag emission covering 3.0 to $4.4 A$ with a resolution of over 1000. A typical streaked spectra from an $\mathrm{Al}$ microdot plasma hested by $1.0 \times 10^{14} \mathrm{~W} / \mathrm{cm}^{2}$ is shown in Figure 16. [Shot \#8433] Beryllium filters 50 to $250 \mu \mathrm{m}$ thick were used to attenuate the $x$-ray flux onto the CsI photocathode. An ITT 4113 image intensifier tube was used to increase the streak tube signal level which was then recorded onto Royal X-Pan film. Each piece of film was preexposed to a calibration step wedge. Therefore, each piece of film was absolutely calibrated. A Joyce-Lobel microdensitometer was used to digitize the XSCS data using a $50 \times 300 \mu \mathrm{m}$ scanning slit. The front end of the spectrometer including the imaging slit, diffraction crystal, and photocathode was carefully shielded from both scattered light and target debris.

There are serious limitations in this instrument mainly due the difficulty in determining the position of the sampled plasma and due to the relatively large region of plasma $(170 \mu \mathrm{m}) \mathrm{x}$-ray data is measured from. Higher spatial resolution (smaller sampled plasma region) can be achieved with increased sensitivity in the XSCS. The signal can be increased by moving in closer to the target without obscuring the line-of-sight of the other instruments. The dense CsI X-ray photocathode could also be replaced with a low-density "fluffy" CsI photocathode with a higher quantum efficiency. A more serious problem is in precisely determining the position of the 
Figure 16. A typical XSCS x-ray spectra (Shot \#8433) from an Al microdot plasma heated by $1.0 \times 10^{14} \mathrm{~W} / \mathrm{cm}^{2}$ representing a 0-170 $\mu \mathrm{m}$ region of plasura from the target surface. The Al K-shell spectra shows the He-like and H-like resonance lines persisting after the heating laser is off. Also, the free-bound recombination continuum is clearly seen. 


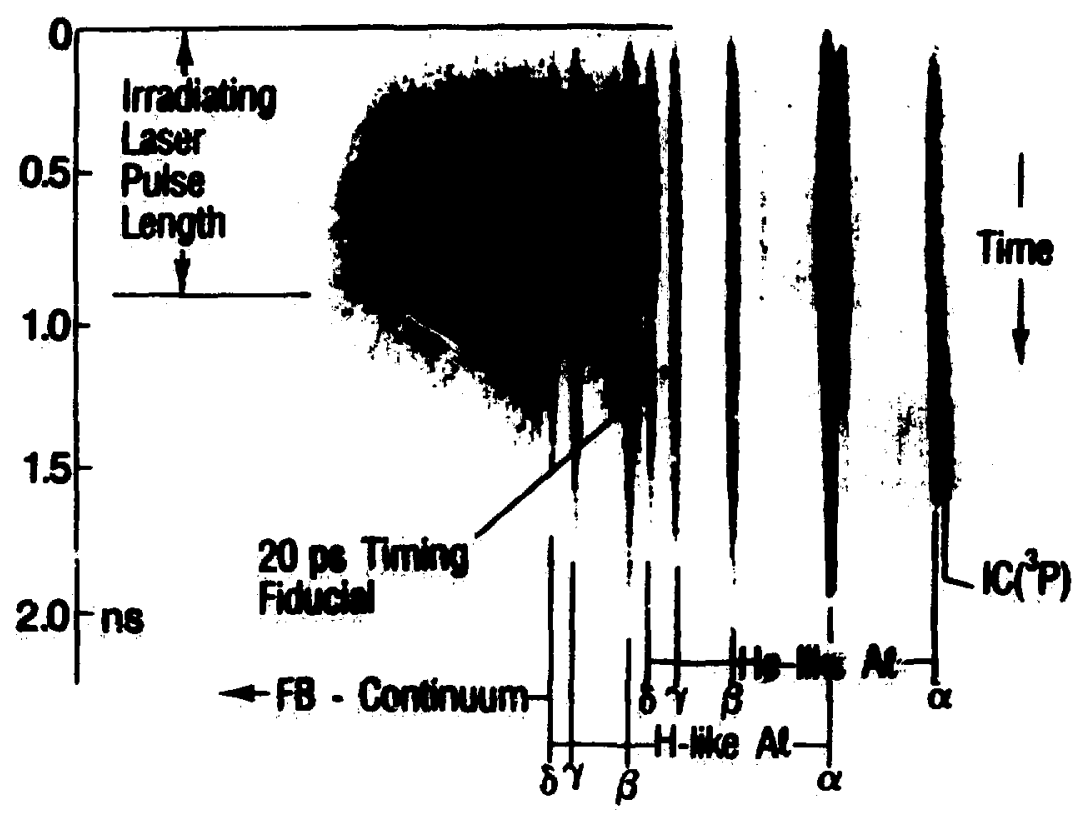

Viewing $\mathrm{N}$ plasma column $\sim 0.170 \mu \mathrm{m}$ from target surface.

Figure 16 
sampled region of plasma. The wire spatial fiducial used in these experiments and described earlier in this section was unreliable. Attempis were made to align the slit using a micro-telescope to sight and align the target and imaging slits from the opposite port. This proved to be very time consuming and equally suspect. Another method for determining the alignment of the XSCS would be by using a pinhole camera precisely located relative to the photocathode slit and mounted on the front end of the streaked camera. The XSCS imaging slit would be used for both the stacak camera and the pinhole camera. A spatial (wire) fiducial would be placed at the center of the photocattode slit, but located such that it casts a shadow only across the pinhole image. Thus, the alignment of the XSCS imaging slit relative to the targe: would be measured on each target shot. 


\subsubsection{4-Frame Holographic Interferometer}

Holographic interferometry provided a non-spectroscopic measurement of the electron density profile with very high spatial and temporal resolution. This technique was first demonstrated by Attwood et al. $(1975 ; 1978)$ to measure the distribution of electrons in high-powered laser-produced plasmas. The method is based on a simple relationship [Pierce (1980)] between the optical index of

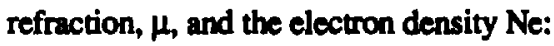

$$
\mu=\left[1-\left(N_{e} / N_{\text {crit }}\right)\right]^{1 / 2}
$$

where $\mathbf{N}_{\text {crit }}$ is the critical density and a function of wavilength,

$$
N_{\text {crit }}=1.1 \times 10^{21}(1.0 \mu \mathrm{m} / \lambda)^{2} \mathrm{~cm}^{-3} \text {. }
$$

Spatial gradients in the electron distribution allow for phase variations in the refractive index of the plasma. Light rays passing through the plasma experience phase changes by some small angular amount proportional to the total transverse density gradient along the ray trajectory. The phase change of the probe beam through the plasma can be measured using holographic interferometry. An ultraviolet probe pulse is used to recond a holographic image of the plasma onto a photographic plate. This image is superimposed onto a previously recorded holographic image of the unperturbed target. This creates a deuble exposure holographic interferogram of the plasma. The holographic image is readily reconstructed using optical light from a CW laser. Since the plasmas measured in these experiments are of cylindrical symmetry, a simple Abel inversion can be performed and th tal phase change along a ray trajectory (total number of 
interference fringes) is directly proportional to the electron density of the sampled plasma. [Attwood et al. (1975; 1978); Pierce (1980)] The electron density is simply derived from the number of interference fringes, $\Delta$, by the relationship,

$$
\Delta \cdot \lambda=(\mu-1) \cdot d
$$

where $\lambda$ is the wavelength of the probe beam and $d$ is the plasma path length. For these experiments, flat targets were irradiated with $10^{13}$ to $10^{14} \mathrm{~W} / \mathrm{cm}^{2}$ of lase: light using the CHROMA laser at KMS Fusion, Inc. The targets were aligned such that the plane of the targets were perpendicular to the heating laser axis. The holographic interferometer probe beam was in the plane of the target. A hot, dense plasma is formed in the first few picoseconds and expands outward away from the target surface. The typical expansion velocity of these high-powered laser-produced plasmas ranged from 1-30 $\times 10^{7} \mathrm{~cm} / \mathrm{s}$. Therefore, to study the details of the electron density distribution of the plasma, the measurement system required a spatial resolution of about $1 \mu \mathrm{m}$ and a temporal resolution of $a$ few tens of picoseconds.

A 4-frame holographic interferometer designed by KMS Fusion was use during the exferiments. [Busch et al. (1985)] The multi-frame capability of the system was a significant improvem int over previous efforts in minimizing the shot-to-shot variations in the data. A set of interferograms taken from two consecutive Al microdot target shots are shown in Figure 17. The instrument used a short 20 ps, $263.0 \mathrm{~nm}$ wavelength laser beam to probe the plasma. The CHROMA oscillator was an actively mode-locked system. It produced a mode-locked train of short $\leq 100$ ps pulses that are $\mathbf{8} \mathrm{ns}$ apart. Two of these pulses were switched out using Pockels cells. One of these pulses was sent to a pulse stacking system which formed the desired pulse shape and was sent down the main amplifier chain where it became the CHROMA heating laser pulse. The second switched out oscillator pulse was 
Figure 17. Sam; : holographic interferograms of an expanding aluminum microdot plasma heated by a $1.0 \mathrm{~ns}$, stacked laser pulse $\left(1.0 \times 10^{14} \mathrm{~W} / \mathrm{cm}^{2}\right)$. These eight interferograms (recorded from Shots $\# 8460$ and $\# 8465$ ) represent 20 ps long time frames. The number of fringe shifts is directly related to the electron density along the path length of the probe beam through the plasma. The interferograms are digitized and analyzed using a simple Abel inversion (assuming cylincrical symmetry) to deduce a two dimensional density profile. 


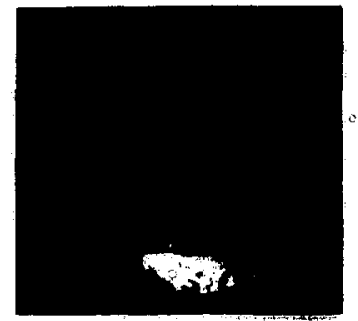

$467 \mathrm{pe}$

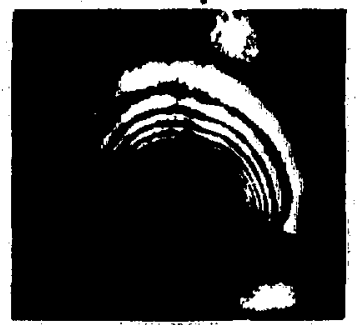

$05 \%$

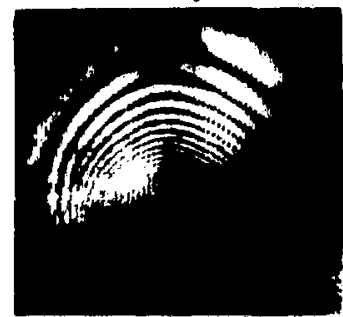

$12.7 \mathrm{p}$

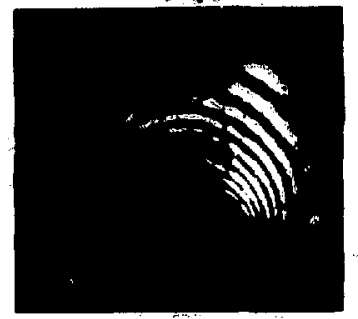

$1657 \mathrm{pe}$

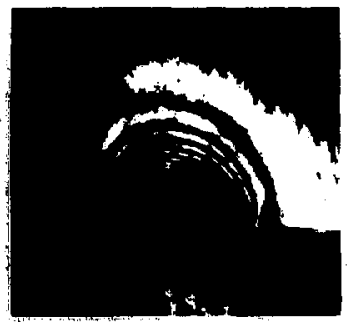

$67 \mathrm{ps}$

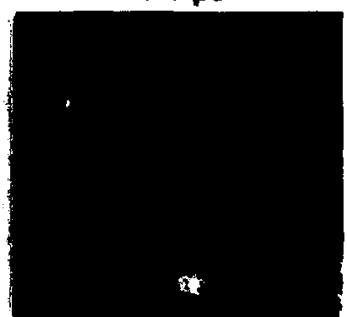

Pap

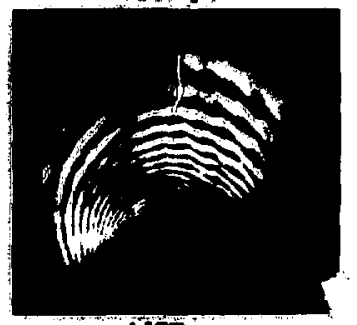

$140 \mathrm{ps}$

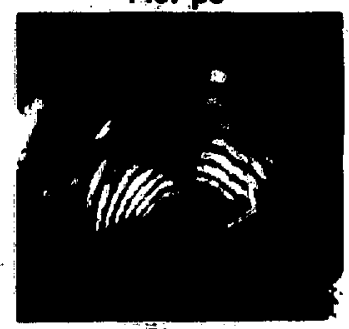

$1657 p$

Figure 17 
injected into a second oscillator cavity where it became the source of the holographic system probe beam. Since both beams originated from the same initial oscillator cavity, the absolute timing between the probe beam and the CHROMA heating pulse was determined from the oscillator cavity dimensions. All other timing differences were due to optical path lengths which were precisely measured to $<5 \mathrm{ps}$.

The probe pulse was then switched out after an appropriate delay time and sent through a regenerative amplifier which shortened the pulse while increasing its amplitude. This probe pulse leaves the regenerative amplifier with an energy of about $500 \mu \mathrm{J}$ and $\mathrm{a} 20 \mathrm{ps}$ pulse width. The pulse widths of both the oscillator and regenerative amplifier pulses were regularly checked using an optical autocorrelator. The probe beam was sent through a rod amplifier which was run at a gain of $20-40$ then sent through a 50\% beam splitter where part of the beam was optically delayed and relayed to the FCXS Auston switches.

The remainder of the $1.05 \mu \mathrm{m}$ beam was frequency doubled to green using a KDP Type II crystal, then frequency coubled a second time to ultraviolet using an ADP crystal. An array of precision optics multiplexed the beam to generate 16 identical beams: two groups of four probe beams sent through the plasma, and two groups of reference pulses. Each holographic interferogram requires four optical pulses: a signal and reference set just before the heating pulse and a second signal and reference set at the chosen probe time during the plasma expansion. (See Figure 18.) The coherence of the system was assured by generating the 16 individual pulses from a single uv pulse. The multiplexer sysiem used beam splitters which had graded coatings to produce pulses of equal energy. The interframe times were adjustable and set at 200 ps for these experiments. Throughout the system, high precision optics, pinhole spatial filters, and long focal length $(f / 1000)$ relay optics were used to maintain beam quality.

The plasma imaging system used large collection optics to collect rays that were 
Figure 18. A schematic diagram depicts the probe and reference beams (four total beams) used to generate a single holographic interferogram (top). For these experiments, a total of 16 beams were used to generate 4 holographic interferograms (bottom). 

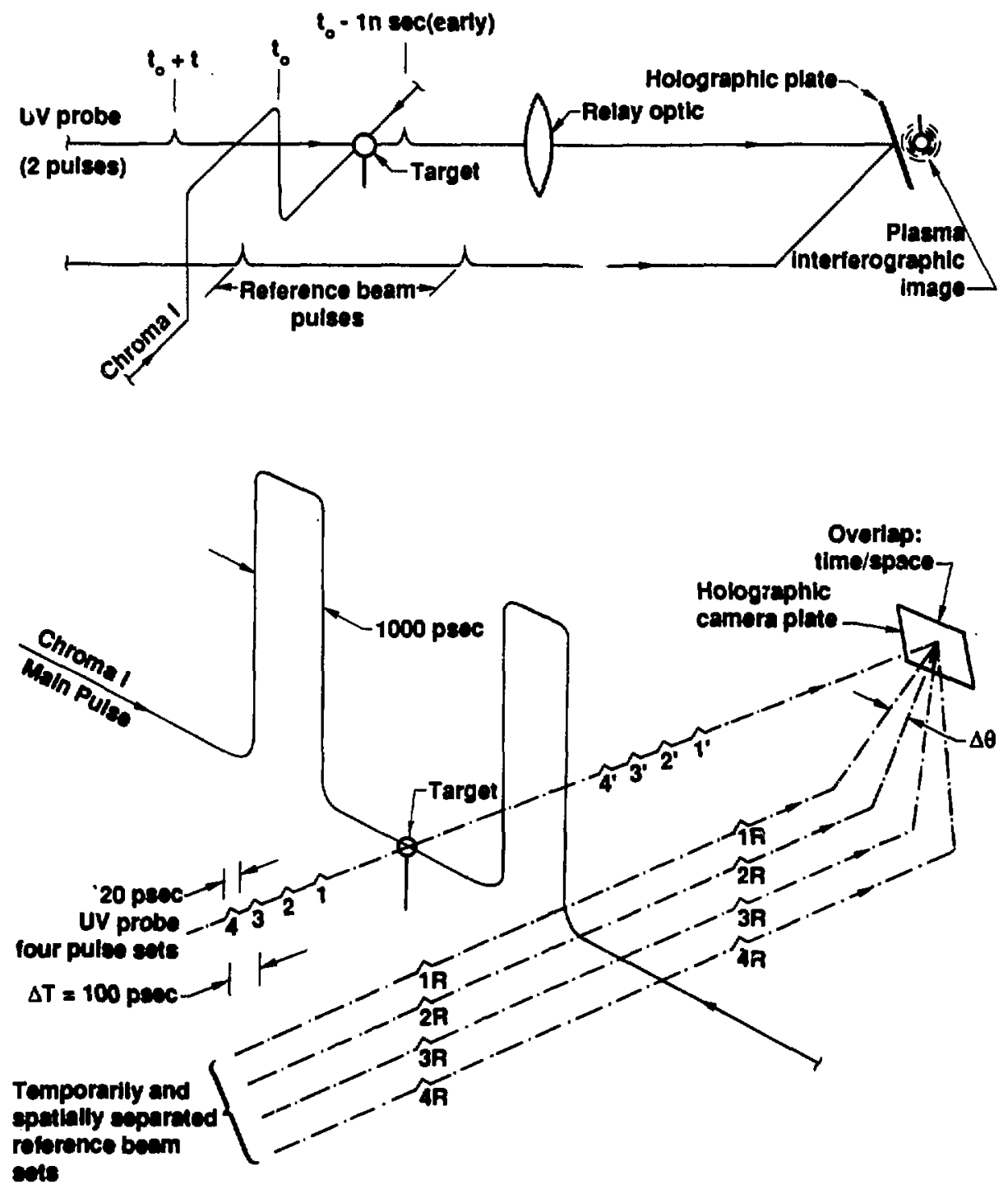

Figure 18 
refracted through the largest possible angle by the plasma. The optical system used a spherical, two-element, $f / 2$ catadioptric lens positioned $11 \mathrm{~cm}$ from the target. Optical emission from the plasma was removed using a broadband, multiple reflection filter. [Busch et al. (1985)] These feakures allowed for measurement of the largest possible densities in the plasma $\left(-2 \times 10^{21} \mathrm{~cm}^{-3}\right)$ with a spatial resolution of $<5 \mu \mathrm{m}$. The $20 \mathrm{ps}$ pulse width minimized plasma motion during the time frame which would otherwise reduce the clarity of interference fringes produced during reconstruction of the holographic interferograms. Thus, the data obtained via holographic interferometry provided an accurate picture of the electron derisity profile in the plasma over a sequence of four distinct sample times. A set of electron density profiles for a typical Al microdot plasma heated by $1.0 \times 10^{14} \mathrm{~W} / \mathrm{cm}^{2}$ is presented in Figure 19. [Shots $\$ 8460$ and $\# 8465$ ] These represent the center-line (axial) densities determined from the Abel inversion of the holographic interferogram. A sample two dimensional contour diagram of the density profile is shown in Figure 20 (Shot \#8464) the Al plasma at 1657 ps after the st:st of the heating laser pulse. It is interesting to note that the density gradients, even at this late probe time, remain predominantly along the axis of the plasma plume with negligible $( \pm 10 \%)$ transverse density gradients over the approximately $100 \mu \mathrm{m}$ central region of plasma corresponding to the expanding $A l$ plasma.

This was a highly complex system that required constant attention during the experiments. Alignment of the optics was constantly monitored as was the performance of the oscillator cavities and regenerative amplifier. The high laser intensities (a few $\mathrm{mJ}, 20 \mathrm{ps}$, approximately $3 \mathrm{~mm}$ diameter beams) presented a high risk for damage to the optical components and also was constantly monitored. Damage to the final catadiopiric collecting lens system due to target debris was also a big concern during the experiments and required occasional cleaning during the series of experiments. Finally, all optical surfaces (approximately 400 in the total 
Figure 19. Time resolved electron density profiles for an aluminum microdot plasma are shown representing a sequence of probe times from 457 to 1857 ps after the start of the $1.0 \mathrm{~ns}$ stacked laser heating pulse at $1.0 \times 10^{14} \mathrm{~W} / \mathrm{cm}^{2}$ (Shots $\# 8460$ and \#8465). These electron density profiles represent the center line or axial density values as determined from the Abel inversion analysis of the data. 

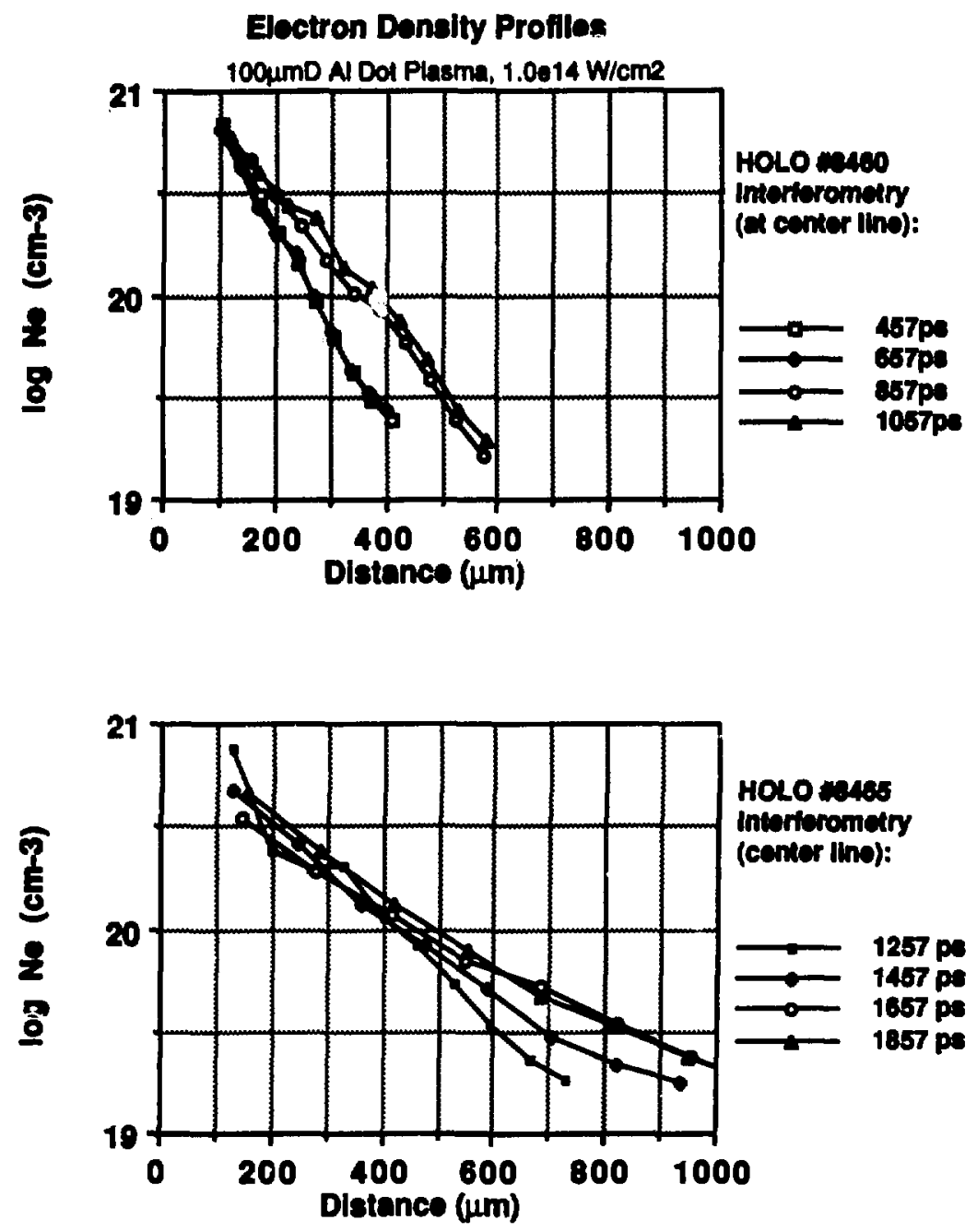

Figure 19 
Figure 20. A typical contour plot of the electron density profile for an Al microdot plasma at $1657 \mathrm{ps}$ after the start of the $1.0 \mathrm{~ns}$ stacked laser heating pulse at $1.0 \mathrm{x}$ $10^{14} \mathrm{~W} / \mathrm{cm}^{2}$ (Shot $\# 8464$ ). The contours correspond to increments of $4.0 \times 10^{19}$ $\mathrm{cm}^{-3}$ in the electron density ranging from $3.9 \times 10^{19} \mathrm{~cm}^{-3}$ (labeled "2") far out in the plasma plume to higher densities closer to the target surface $2.8 \times 10^{20} \mathrm{~cm}^{-3}$ (labeled "8"). This figure indicates that the electron density profile about the central $100 \mu \mathrm{m}$ region of plasma (relative to the heating laser axis) is fairly isodense. 


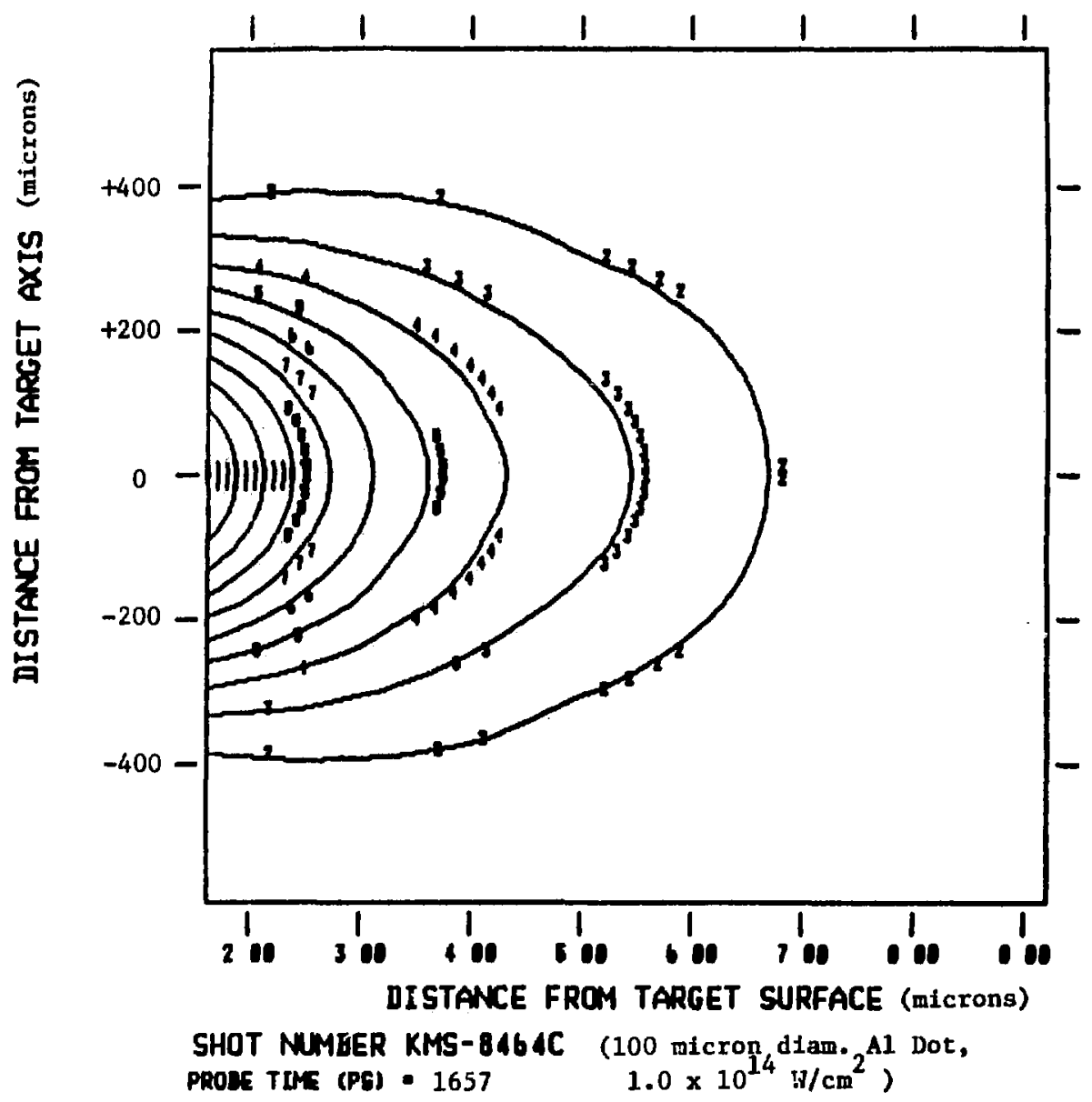

FIgure 20 
system) had to be maintained and kept dust free to allow for maximum beam quality and transmission.

\subsubsection{Other Plasma and Laser Diagnostics}

Additional plasma and laser diagnostics were used to study these laser produced plasma. These plasma diagnostics were more contemporary instruments that have been used in the majority of past laser plasmas experiments. They included 2 space resolved, time-integrated flat crystal mini-spectrometers: one using a high dispersion/high resolution diffraction crystal (ADP or PET) and the other set up with a lower dispersion crystal (KAP) as a survey instrument. These spectrometers used $50 \mu \mathrm{m}$ wide spatial imaging slits and $50 \mu \mathrm{m}$ thick Be filters. The slit sizes (50 $\mu \mathrm{m}$ wide) were selected to provide a similar spatial resolution to that obtained with the FCXS. These time-integrated spectrometers were normally operated at a magnification of 6 to 7 and provided spatial resolution of approximately $70 \mu \mathrm{m}$. This would allow for a direct comparison of time resolved to time-integrated spectral data The time-integrated data was digitized with the Perkin-Elmer PDS scanning densitometer using a $10 \times 50 \mu \mathrm{m}$ scanning slit. Two $\times$-ray pinhole cameras recorded the focal spot size and provided information on the uniformity of the plasma. The $x$-ray film response, crystal reflectivity, and filter transmission were removed using the calibration curves of Rockett et al. (1985; 1986), Burek (1976), and Henke et al. (1981; 1982; 1986), respectively.

An optical streak camera recorded the CHROMA pulse shape for each shot. An optical autocorrelator was used to measure the tempoial pulse width of the probe beam out of the regenerative amplifier. The probe pulse was also monitored occasionally for prepulse as described earlier. An array of photodiodes located inside the target chamber recorded the reflected laser light off of the target. The 
CHROMA optics were calibrated aid the total absorbed energy for each shot was determined. These laser diagnostics were critical in parameterizing the actual laser heating conditions for these plasmas and in assessing similar far get shots used in this work. 


\section{Chapter 3. Important Properties of Leser-Produced Plasmas}

In this chapter, I briefly review the standard equilibrium plasma modeling methods used to interpret spectral data measured from typical laboratory plasma sources. In section 3.1, I outline the various plasma regimes in which some atomic processes are typically neglected to simplifying the theoretical model required to simulate these laboratory plasmas. In section 3.2, I describe the collisional radiative equilibrium "RATION" atomic model which was used to interpret the aluminum and magnesium K-shell spectral data measured in these experiments. In section 3.3, I present a short discussion on plasma opacity effects which distort the measured datu and often confuses the interpretation of the data. In section 3.4, I review some important plasma parameters which were considered throughout the experiments and are fundamental to high powered laser-produced plasmas.

\subsection{A General Description of Plasma Models}

The characterization of laboratory plasmas is typically based on the interpretation of measured $x$-ray emission prodiced by the plasmas. This data analysis is highly dependent upon the atomic model used as well as assumptions made concerning the hydrodynamics of the plasma. In order to extract meaningful information characteristic to the plasma (such as the electron temperature or density), the detailed tomic physics governing the ion population kinetics must be properly included in the theoretical model. Therefore, a brief review of the various atomic models used to study typical laboratory plasmas will be presented here.

For very high density plasmas such as those created in laser inertial confinement 
fusion experiments $\left(n_{e} \geq 10^{23} \mathrm{~cm}^{-3}\right.$ ), it is often assumed that the distribution of the electrons is determined exclusively by particle collisions. These collisions occur sufficiently rapid enough for the electron distribution to respond instantaneously to any change in the local plasma conditions. In such circumstances, each atomic process and its inverse occur at equal rates govemed by the principle of detailed balance. The population distribution is determined by the statistical equipartition of electrons among all possible states. This situation is called "Local Thermodynamic Equilibrium" (LTE) and the free electrons have a velocity distribution (Maxwellian distribution) described by the following expression:

$$
P(v)=n_{e}(v) \cdot d v=4 \pi n_{e} \cdot\left(\frac{m}{2 \pi k T_{e}}\right)^{3 / 2} \cdot v^{2} \cdot \exp \left[\frac{-m v^{2}}{2 k T_{e}}\right] \cdot d v
$$

where $P(v)$ is the probability of having $n_{e}(v) \cdot d v$ electrons with a velocity between

$v$ and $v+d v$ at a total electron density $n_{e}$ and temperature $T_{e}$. The population distribution for bound atomic levels are determined from the Boltzmann and Saha equations, respectively:

$$
\begin{gathered}
\frac{n(j)}{n\left(j^{\prime}\right)}=\frac{g(j)}{g\left(j^{\prime}\right)} \cdot \exp \left[\frac{\chi\left(j, j^{\prime}\right)}{k T_{e}}\right] \\
\frac{n_{0}(z+1) \cdot n_{e}}{n_{0}(z)}=\frac{g_{0}(z+1)}{g_{0}(z)} \cdot 2 \cdot\left(\frac{2 \pi m k T_{e}}{h^{2}}\right)^{3 / 2} \cdot \exp \left[\frac{\chi(z, 0)}{k T_{e}}\right]
\end{gathered}
$$

where $n(j), n\left(j^{\prime}\right), n_{0}(z+1)$, and $n_{0}(z)$ are the population densities of the various levels designated by their quantum numbers $j, j$, and 0 (corresponding to the ground level) and the ion charges $z$ and $z+1$. The term $g_{j}(z)$ is the statistical weight of the 
designated level. $\chi\left(j j^{\prime}\right)$ is the energy difference between the levels $j$ and $j^{\prime}$, and $\chi(z, 0)$ is the ionization potential of the ion of charge $\mathrm{z}$ in the ground level. In applying this form of the Saha equation, I have assumed that the majority of the population of any particular ionization state exists in the ground level. The ionization distribution as well as the kinetics goveming individual atomic level populations are affected by collisional ionization, electron-impact excitation, and collisional recombination (3-body recombination).

For low density plasmas such as those existing in the solar corona or in tokamak fusion devices (typically $\mathrm{n}_{\mathrm{e}} \leq 10^{15} \mathrm{~cm}^{-3}$ ), a Coronal Model is typically applied in which collisional ionization and excitation, and radiative recombination populate the excited state levels, and spontaneous radiative decays are the main depopulating mechanism governing the level populations in the plasma. In such a low density, coronal plasma, the rate equations describing the ionization distribution can be written as:

$$
\begin{aligned}
\frac{d n_{0}(z)}{d t} & =n_{0}(z-1) \cdot n_{e} \cdot\left\langle v \cdot \sigma_{i o n}\left(z-1, T_{e}\right)>-n_{0}(z) \cdot n_{e} \cdot\left\langle v \cdot \sigma_{i o n}(z, T e)\right.\right. \\
& -n_{0}(z) \cdot n_{e} \cdot \alpha_{10 c}\left(z, T_{e}\right)+n_{0}(z+1) \cdot n_{e} \cdot \alpha_{10 c}\left(z+1, T_{e}\right)
\end{aligned}
$$

where $\alpha_{\text {rec }}\left(z, T_{e}\right)$ is the radiative recombination coefficient and $\left\langle v \cdot \sigma_{i o n}\left(z, T_{e}\right)\right\rangle$ is the collisional ionization rate of the ground state of charge $\mathrm{Z}$ and temperature $\mathrm{T}_{e}$. Similarly, the population densities of the individual atomic levels can be determined from a system of rate equations (for each level $j$ and for each charge state $Z$ ) described by collisional excitations from the ground state and spontaneous radiative decays of the excited levels: 


$$
\frac{\operatorname{dn}(z, j)}{d t}=n_{e} \cdot n_{0}(z) \cdot<v \cdot \sigma_{e x c}\left(T_{e}, j\right)>-n(z, j) \cdot \sum_{j<j^{\prime}} A_{r}\left(j^{\prime} \rightarrow j\right)
$$

where $A_{r}\left(j^{\prime} \rightarrow j\right)$ is the radiative decay rate from state $j^{\prime}$ to state $j$. This rate equation neglects losses and gains due to recombination and ionization processes. Again, the free electrons are assumed to have a Maxwellian velocity distribution. In a steady state (equilibrium) coronal plasma, the rate equations for both the ionization distribution and the level populations can be simplified by balancing the collisional ionizations and excitations by the radiative resombinations and spontaneous decays.

$$
\begin{gathered}
\frac{n_{0}(z) \cdot n_{e}}{n_{0}(z+1) \cdot n_{e}}=\frac{\alpha_{1 e c}\left(z+1, T_{e}\right)}{\left\langle v \cdot \sigma_{i o n}\left(z, T_{e}\right)\right\rangle} \\
n_{e} \cdot n_{0}(z) \cdot\left\langle v \cdot \sigma_{e x c}\left(T_{e}, j\right\rangle>n(z, j) \cdot \sum_{j<j} A_{r}\left(j^{\prime} \rightarrow j\right)\right.
\end{gathered}
$$

Both the LTE and Coronal models are greatly simplified by assuming that the plasma is optically thin and that the emitted radiation escapes from the plasma without interacting with it. The opacity of the plasma can be a very important process which affects both the escape probability of the observed spectral emission as well as affecting the overall population dynamics of the plasma. The opacity effects are discussed later in this chapter.

\subsection{Collisional Radiative Models}

For the intermediate type plasma corresponding to electron densities typically $10^{19}$ $\leq n_{e} \leq 10^{22} \mathrm{~cm}^{-3}$, radiative and collisional processes are often equally important and 
cannot be neglected. These plasma are often characterized by very detailed Collisional Radiative Models (CR). In the CR Model, the rate equations describing the population kinetics of level $\mathrm{n}_{\mathrm{j}}(\mathbf{z})$ of charge $\mathrm{z}$ must include collisional mixing (excitations and decays) to all other levels in that charge state and radiative decays from higher lying excited states (again, assuming an optically thin plasma). All recombination processes to and from ions of charge $(z+1)$ as well as ionization processes to and from ions of charge (z-1) must also be accounted for. The resulting system of rate equations can be extremely large and complex. For example, there will be a rate equation of the following form for each level $n_{j}(z)$, where:

$$
\begin{aligned}
\frac{d n_{j}(z)}{d t} & =-n_{j}(z) \cdot\left\{n_{e} \cdot\left\langle v \cdot \sigma_{i o n}\left(z, T_{e}\right)\right\rangle_{j}+n_{e} \cdot \sum_{j \neq k}\left\langle v \cdot \sigma_{e x c}\left(z, T_{e}\right)\right\rangle_{j k}\right. \\
& \left.+\sum_{k<j} A_{r}(j-k)\right\}+n_{e} \cdot \sum_{j \neq k} n_{k}(z) \cdot\left\langle v \cdot \sigma_{e x c}\left(z, T_{e}\right)\right\rangle_{k j}+\sum_{b j} n_{k}(z) \cdot A_{r}(k \rightarrow j) \\
& +n_{e} \cdot \sum_{k(z+1)} n_{k}(z+1) \cdot\left\{n_{e} \cdot R_{k \rightarrow j(z)}^{3 B}\left(z+1, T_{e}\right)+\alpha_{k \rightarrow j(z)}^{r e c}\left(z+1, T_{e}\right)\right\} \\
& +n_{e} \cdot \sum_{k(z-1)} n_{k}(z-1) \cdot<v \cdot \sigma_{i o n}\left(z-1, T_{e}\right)>_{k \rightarrow j(z)}
\end{aligned}
$$

where for temperature $T_{e}:\left\langle v \cdot \sigma_{e x c}\left(z, T_{e}\right)\right\rangle_{j k}$ is the collisional excitation (or deexcitation) rate for transitions between states $j$ and $k$ of charge $z ;\left\langle v \cdot \sigma_{i o n}\left(z, T_{e}\right)\right\rangle_{j}$ is the collisional ionization rate out of the state $\mathrm{j}$ of charge $\mathrm{z} ;\left\langle u \cdot \sigma_{\mathrm{ion}}\left(2-1, \mathrm{~T}_{\mathrm{e}}\right)\right\rangle_{\mathrm{k} \rightarrow \mathrm{j}(\mathrm{z})}$ is the collisional ionization rate or the level $n_{k}(z-1)$ feeding level $n_{j}(z)$; and where $\alpha^{\mathrm{rec}} \mathrm{c}_{\mathrm{k} \rightarrow \mathrm{j}}\left(z+1, \mathrm{~T}_{e}\right)$ and $\mathrm{R}^{3 \mathrm{~B}_{\mathrm{k}} \rightarrow \mathrm{j}}(\mathrm{z}+1)$ are the total radiative and 3-body recombination rates, respectively, feeding the level $n_{j}(z)$. There can also be losses due to 
recombinations of the level $n_{j}(z)$ but this has been omitted here. Finally, the last two summations in the above rate equation can usually be replaced by single terms by considering the recombination and ionization contributions only from the ground levels of the neighboring charge states. This is valid (and typically is) if the majority of the population of any charge state is in the ground state.

In presenting rate equations for both the Coronal Model and the Collisional Radiative Model, I have neglected to include contributions due to a very important atomic process, dielectronic recombination, which must also be included in the rate equations. It is a complex, multi-step process: first, an electron must be resonantly captured creating a doubly excited state; this is then followed by a radiative decay by one of the excited electrons. Therefore, the expression describing the contribution to each level $n_{j}(z)$ due to dielectronic recombination must include a double summation over all states of charge $\mathrm{z}+1$ (allowing for the electron capture) and for all possible doubly excited states of charge $z$ (in which a radiative decay can lead to state $j$ ). For instance, the dielectronic recombination from a He-like ground state to a state $\left(1 s^{2}, n\right)$ of a Li-like ion can be represented by

$$
X^{Z-1}\left(1 s^{2}\right)+e^{-} \Leftrightarrow X^{Z}\left(1 s, n^{\prime} l^{\prime}, n^{\prime \prime} l^{\prime \prime}\right) \rightarrow X^{Z}\left(1 s^{2}, n\right)+h v
$$

A source term due to dielectronic recombination to the population of level $n_{j}(z)$ can be described by an expression of the form:

$$
+n_{e} \cdot \sum_{i} n_{i}(z+1) \cdot \alpha_{D R}(z+1, i)
$$

where $\alpha_{D R}(z+1, i)$ is the dielectronic recombination coefficient for each state $\mathrm{n}_{\mathrm{i}}(\mathrm{z}+1)$ which may undergo dielectronic recombination and feed the state $\mathrm{n}_{\mathrm{j}}(\mathrm{z})$. This certainly is not a complete description of the very complex dielectronic recombination 
process, but instead is an attempt to briefly account for this atomic process. For a detailed description of dielectronic recombination, I refer the reader to several excellent references on the subject by Chen (1985; 1986a; 1986b), Duston et al. (1983), Dubau et al. (1980; 1981), Gabriel et al. (1972a; 1972b; 1972c).

Dielectronic rates used to analyze the density dependence of the " $\mathrm{He} / \mathrm{dC}$ " line intensity ratio were provided by M.H. Chen (1988) using a multiconfigurational Dirac-Fock (MCDF) model to evaluate the detailed energies, and Auger and radiative decay rates. To illustrate the complexity of the calculation, I've shown Chen's expression for the total dielectronic recombination coefficient (isolated resonance approximation) out of the state $n_{i}(z+1)$ :

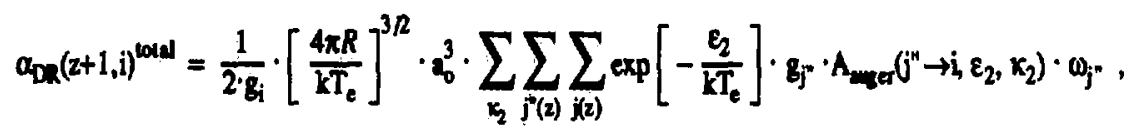

with $\omega_{\mathrm{j}}$ representing the fluorescence yield of the doubly excited state $\mathrm{j}^{\prime \prime}$ to the final state $j$ including higher-order processes such as cascades from unstable levels by the emission of an additional photon or electron. The fluorescence yield can be written as

$$
\omega_{j^{\prime \prime}}=\frac{1}{\Gamma_{j^{\prime \prime}}} \cdot \sum_{j(z)}\left[A_{r}\left(j^{\prime \prime} \rightarrow j\right)+\sum_{k} \frac{A_{J}\left(j^{\prime \prime} \rightarrow k\right)}{\Gamma_{k}} \cdot A_{r}(k \rightarrow j)\right]
$$

where $\Gamma_{j^{n}}=\Gamma_{r}\left(j^{\prime \prime}\right)+\Gamma_{a}\left(j^{\prime \prime}\right)$. Here, $\Gamma_{i}$ and $\Gamma_{j^{n}}$ are the statistical weights for the charge states z-1 and z, respectively, $R$ is the Rydberg energy, and $a_{0}$ is the Bohr radius. Also, $A_{\text {auger }}\left(j^{\prime \prime} \rightarrow i, \varepsilon_{2}, k_{2}\right)$ is the rate for Auger transitions between the doubly excited state $j "(z)$ and initial state $i(z-1)$ of the recombining ion, for a 
free-electrons of energy $\varepsilon_{2}$ and relativistic quantum number of $x_{2}=\left(l_{2}-j_{2}\right) \cdot\left(2 \cdot j_{2}\right.$ +1 ). Thit free electron are assumed to have a Maxwellian velocity distribution. And $A_{x}(j " \rightarrow j)$ is the radiative decay rate from the doubly excited state $j^{\prime \prime}$ to the final state $j$; $\Gamma_{\mathrm{I}}$ and $\Gamma_{\mathrm{a}}$ are the total radiative and Auger widths, respectively. Similarly, a loss term for a level $n_{j}(z)$ can also be described by an expression which includes a double summation for all doubly exilited states of charge z-1 and final states of $n_{j}(z-1)$.

For the Collisional Radiative regime, several approximations can be made to simplify the system of rate equations. Often, only a small number of charge states are modeled with great detail such as $\mathrm{H}$-like, He-like, and maybe Li-like depending on the particular state of ionization of interest. Also, a reasonably good assumption is often made by noting that high lying excited levels (those with increasing quantum numbers) are often closeiy spaced in energy and the probability for collisional processes between such levels exceeds the probability for radiative decays. Therefore, these levels are often considered to be in LTE and their populations governed by the Saha (or Boltzmann) relationship.

The laser-produced plasmas studied in the present work fall in this Collisional Radiative regime. Several different analytical methods, based on the atomic processes characteristic of a CR plasma, have been applied in the interpretation of the measured spectral data. These include a collisional radiative equilibrium, "CRE" model for K-shell plasmas, a more detailed, quasi-steady state collisional radiative K-shell model, and two quasi-steady state collisional radiative L-shell models for neon-like ions. The quasi-steady state models focus on a small range of ionization states treating the relative charge state fractions as a free parameter. These quasi-stendy state models are described in Chapter 4. The "CRE" atomic model 
assumes an equilibrium charge state distribution and is described below.

\subsection{1. "RATION" Collisional Radiative Equilibrium Model}

"RATION/RATSHOW/SPECTRA" (referred to as "RATION" throughout the remainder of this dissertation) is an example of a detailed, steady state model in which the ionization distribution and level population kinetics are assumed to be in Collisional Radiative Equilibrium (CRE). The "RATION" model was developed by R.W. Lee et al. (1985). I have used a version of the code modified by R. Cauble (1987) to run on the CRAY I computer on the LLNL OCTOPUS computer system and which accounts for line opacities using a simple escape factor approximation. The model was used to analyze the spectral data presented in this thesis and is outlined below. A detailed description of the "RATION" code can be found in R.W. Lee et al. (1985) and Kauffman et al. (1987).

"RATION" and "RATSHOW" are the first two parts of a semi-empirical atomic modeling code. The code was developed to model and analyze the $\mathrm{x}$-ray emission from highly ionized plasmas such as those produced by Z-pinch machines or in laser plasmas. This model focuses on the atomic physics of low-Z, $\mathrm{K}$-shell systems (bare, H-like, $\mathrm{He}$-like and some Li-like ions). The model contains only a very simple treatment of the radiation transpost in the specified plasma volume and there is no modeling of the hydrodynamics of the plasma. The code was designed specifically for analyzing or modeling spectral data from plasma sources with small spatial and temporal gradients. For cases in which there are gradients or opacity effects, the "RATION" code provides a "bracket" on the plasma parameters determined by the experimental data.

"RATION" is a rate equation model which is used to calculate the populations of the various $\mathrm{H}$-like, $\mathrm{He}$-like, and some Li-like states. The model constructs and 
solves a rate equation matrix in a "collisional radiative equilibrium" (CRE) and includes all ionization states from neutral to fully stripped. For $\mathrm{H}$-like, the model includes the ground state as well as the hydrogenic states $(1 \mathrm{~s}-\mathrm{np})$ for $\mathrm{n}=1$ to 5 . For He-like, the model includes the ground state and the four (1s $2 l$ excited states explicitly; the higher excited states are lumped into one state of the form (1snl); and six doubly excited states of the form $(2 / 2 /)$ are included explicitly. For Li-like, the model includes the ground state $1 \mathrm{~s}^{2} 2 \mathrm{~s}(2 \mathrm{~S})$, the first excited state $1 \mathrm{~s}^{2} 2 \mathrm{p}(2 \mathrm{P})$ and the doubly excited states of the form $1 \mathrm{~s} 2 / 2 l^{\prime}(2 \mathrm{~L})$. The model includes most atomic processes including electron-impact excitation, desexcitation and ionization, three-body recombination, dielectronic recombination and autoionization of cisubly excited states, radiative recombination, and photoexcitation and photoionication. Sources for the atomic data used in this code (ie. energy levels and rates) are taken from a range of sources. Processes not included are accounted for using detailed balance. As an example, I have listed in Table 1, hydrogenic rates of Weisheit et al. (1976) and Dubau et al. (1980) which may be included in an atomic model similar to the "RATION" model. These rates are not used in "RATION", but are presented as simple exampies of the formulae that are often used to compute rates for the various atomic processes.

The "RATSHOW" code is a line ratio program which uses the population files produced by the rate equation model to plot line-intensity ratios as a function of the electron density, electron temperature, or both. In Figure 21, I present a set of line intensity plots produced by the "RATION" and "RATSHOW" calculations for aluminum ( $Z=13)$. Both the optically thin (left) and optically thick [assuming one dimensional, $50 \mu \mathrm{m}$ thick plasma] (right) $\mathrm{CRE}$ line intensity ratio plots are presented. They include: a) the He-like $\left[1 \mathrm{~s}^{2}\left({ }^{1} \mathrm{~S}_{0}\right)-1 \mathrm{~s} 2 \mathrm{p}\left({ }^{1} \mathrm{P}_{1}\right)\right]$ "He${ }_{\alpha}$ " resonance line to He-like $\left[1 \mathrm{~s}^{2}\left({ }^{1} \mathrm{~S}_{0}\right)-1 \mathrm{~s} 2 \mathrm{p}\left({ }^{3} \mathrm{P}_{1}\right)\right]$ "IC" intercombination line ratio versus the 
Table 1. Typicai atomic collisional and radiative (hydrogenic) rates are listed and provide a simple estimate of the Z-scaling of these various rates. 
Table 1

\section{ATOMTCRATE COEFEICIENTS}

Process:

1. Radiative

$$
\alpha_{n}=5.2 \times 10^{-14} \cdot Z \cdot \chi_{\hbar}^{1 / 2} \cdot \Phi\left(\chi_{n}\right) \quad\left[\mathrm{cm}^{3} \cdot \sec ^{-1}\right]
$$

Recombination:

$X^{Z}+e \rightarrow X_{n}^{(Z-1)}+h v$

2. Collisional

$$
C_{n v}=8.7 \times 10^{-3} \cdot\left(\frac{n}{Z}\right)^{3} \chi_{n}^{1 / 2} \cdot \exp \left(-\chi_{n}\right) \cdot\left[1-\Phi\left(\chi_{n}\right)\right]\left[\mathrm{cm}^{3} \cdot \sec ^{-1}\right]
$$

Ionization:

$$
x_{n}^{(Z-1)}+e \rightarrow X^{Z}+2 e
$$

3. 3-Body

$$
C_{v n}=5.6 \times 10^{-31} \cdot\left(\frac{n}{z}\right)^{6} \cdot x_{n}^{2} \cdot n^{2} \cdot\left[1-\Phi\left(\chi_{n}\right)\right]\left[\mathrm{cm}^{6} \cdot \sec ^{-1}\right]
$$

Recombisation:

$X^{Z}+e+e \rightarrow X_{n}^{(2-1)}+e$

4. Collisional

$$
C_{n m}=6.4 \times 10^{-8} \cdot\left(\frac{n}{z}\right)^{3} x_{n}^{1 / 2} \cdot\left(\frac{m^{2}}{m^{2}-n^{2}}\right) .
$$

Excitation:

$$
\times f_{n m} \cdot \exp \left(x_{m}-\chi_{n}\right)\left[\mathrm{cm}^{3} \cdot \sec ^{-1}\right]
$$

$X_{\mathrm{a}}^{(\mathrm{Z}-1)}+e \rightarrow x_{\mathrm{m}}^{(\mathrm{Z}-1)}+e$

5. Radiative Decay $\quad A_{\operatorname{mn}}=8.0 \times 10^{9} \cdot\left(\frac{Z}{m}\right)^{4} \cdot\left(\frac{m^{2}-n^{2}}{m n}\right) \cdot f_{n m}\left[\sec ^{-1}\right]$

$$
\mathbf{X}_{m}^{(Z-1)} \rightarrow X_{n}^{(Z-1)}+h y
$$

6. Dielectronic Recombination:

$$
\begin{aligned}
& X^{(Z-1)}(i)+e \Leftrightarrow X^{Z}\left(j^{\prime \prime}\right) \rightarrow X^{Z}(j)+h v \\
& \alpha_{D R}(i, j)=3.3 \times 10^{-24} \cdot\left[\frac{E_{k}}{k T_{e}}\right]^{\beta / 2} \cdot \frac{g_{i}}{g_{j}} \cdot \exp \left[-\frac{\Delta E_{i j}}{k T_{e}}\right] \cdot A_{i j}^{210}\left[\mathrm{~cm}^{3} \cdot \sec ^{-1}\right]
\end{aligned}
$$

where $\chi_{n}=157890 \cdot Z^{2} \cdot\left[n^{2} \cdot T\left({ }^{0} K\right)\right]^{-1}$,

$\Phi(x) \equiv x \cdot \exp (x) \cdot E_{1}(x), \quad\left[E_{1}\right.$ is the exponential integral],

$f_{\mathrm{mn}}=$ hydrogenic oscillator strength,

$E_{H} \equiv 13.6 \mathrm{eV}$. 
Figure 21. The theoretical line intensity ratios have been calculated for an optically thin (left) and [50 $\mu \mathrm{m}]$ optically thick (right) Al plasma using the "RATION" collisional radiative equilibrium (CRE) model. These line intensity ratios include: a) the "He $/ \mathrm{IC}$ " line ratio, " $\alpha$ ", as a function of the electron density; b) the "Heg/ $/ \mathrm{C}$ " line ratio as a function of the electron density; c) the "Hed $/(\mathrm{IC}+\mathrm{mn}+\mathrm{st})$ " line ratio as a function of the electron density; d)the " $\mathrm{He} / \mathrm{L} \mathrm{y}_{\beta}$ " line ratio us a function of the electron temperature; $e)$ the " $\left.\mathrm{Ly}_{d} / 1 \mathrm{~s} 2 \mathrm{p}\left({ }^{1} \mathrm{P}_{1}\right)-1 \mathrm{p}^{2}(\mathrm{ID})\right]$ dielectronic satellite" line ratio as a function of the electron temperature; $f)$ the "Ly $/\left[1 \operatorname{s}_{2} p\left({ }^{1} P_{1}\right)-1 p^{2}\left({ }^{1} D\right)\right]$ dielectronic satellite" line ratio as a function of the electron temperature; and $\mathrm{g}$ ) the "He $/ \mathrm{He}_{\beta}$ " (eft) and the "Heg/Hep" (right) line ratios as a functions of the electron density. [Lee et al. (1985); Cauble (1987)] 


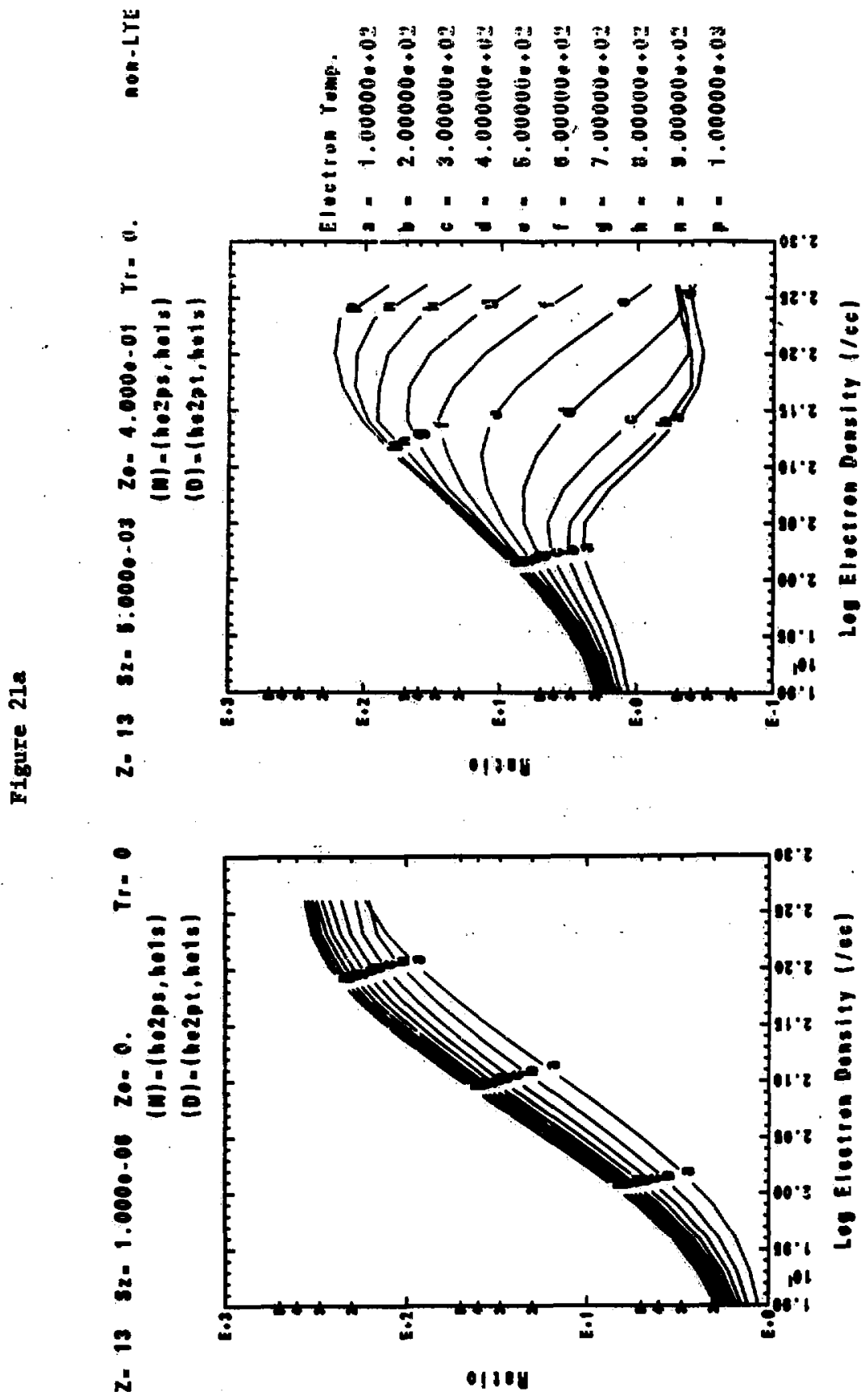



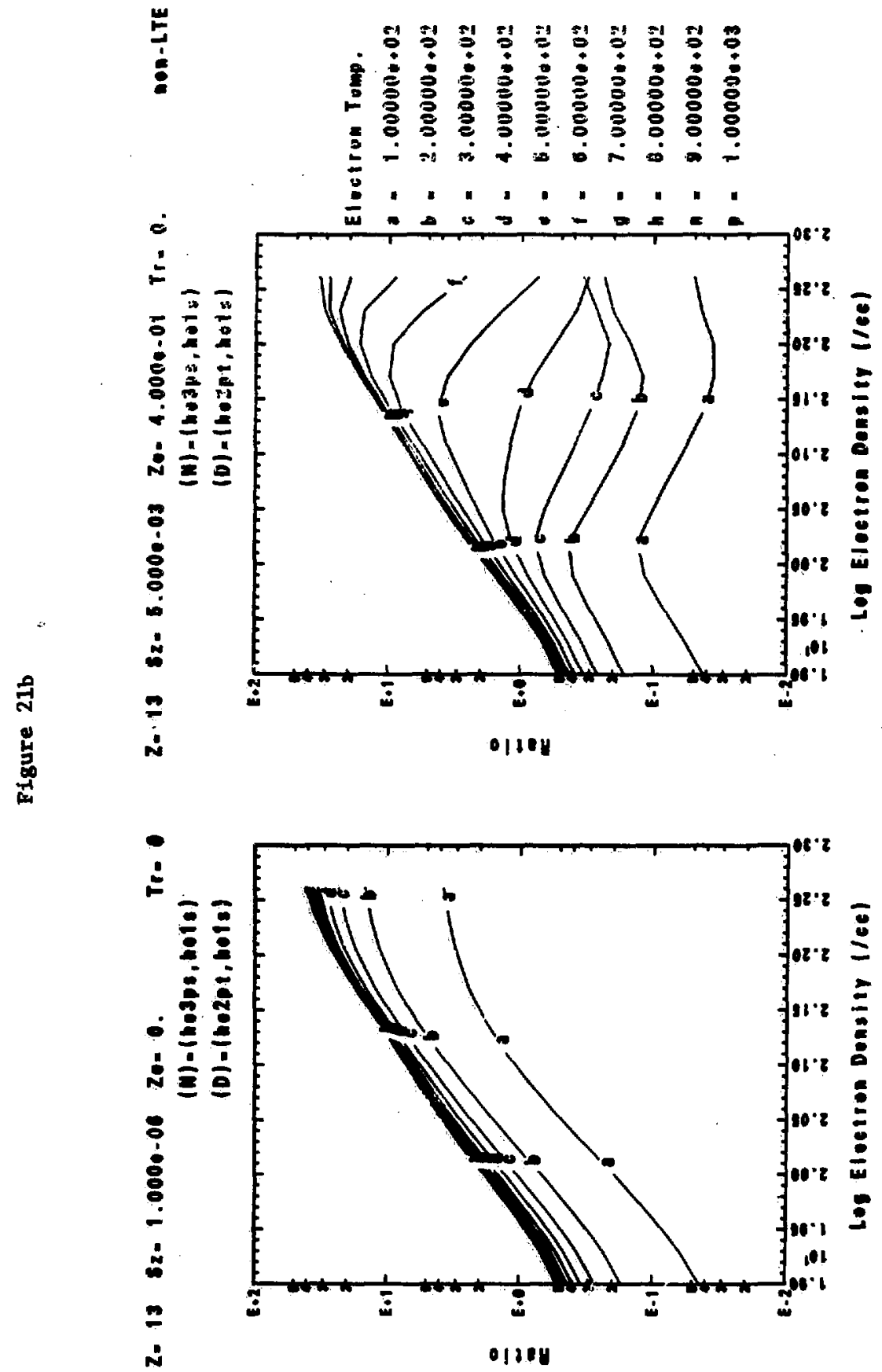
불
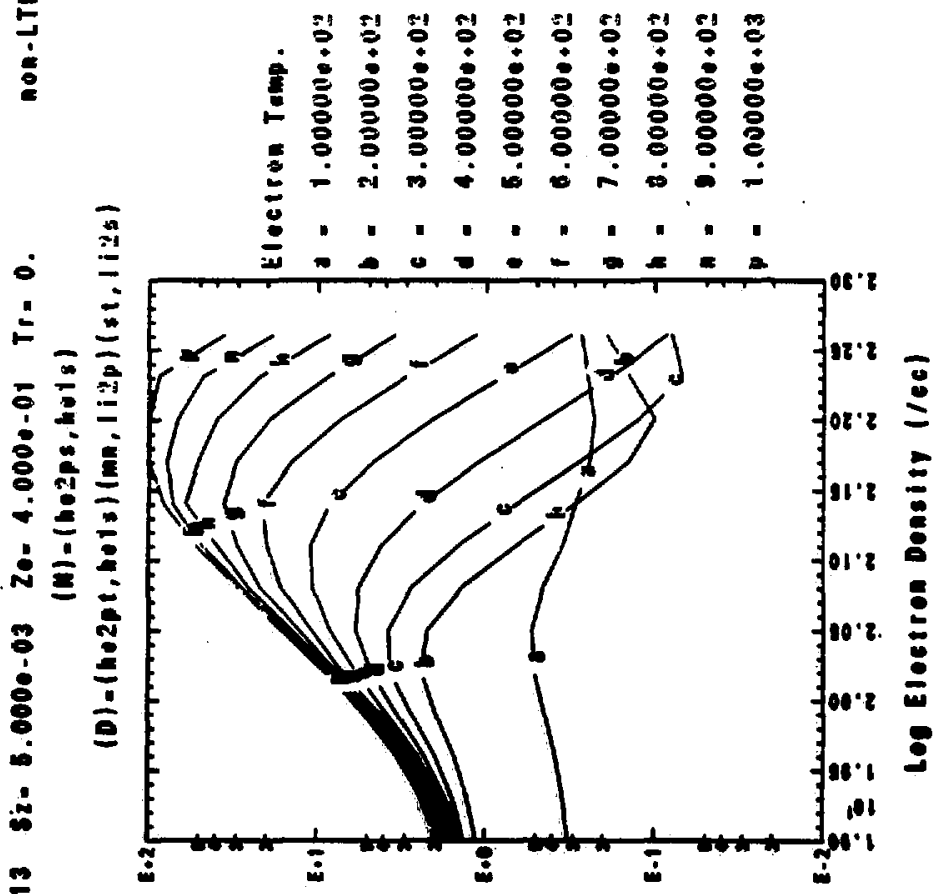

119y

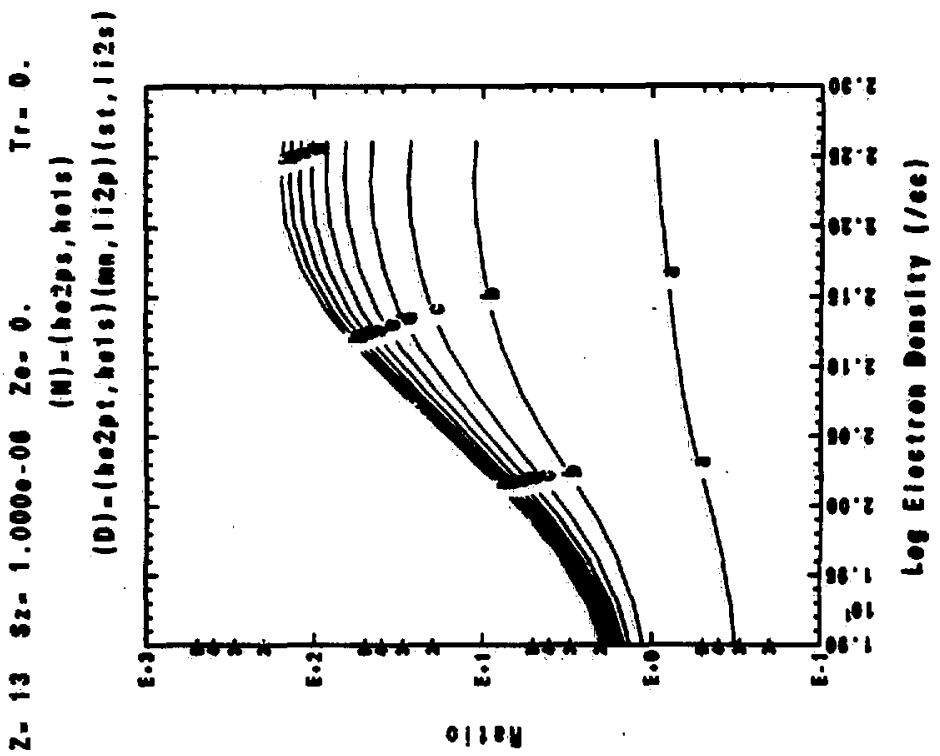


Plgure 21d

Z- $13 \quad s z=1.000-00 \quad z e=0$.

(ii)-(heaps, thets)

(Dlo(try, hyt)

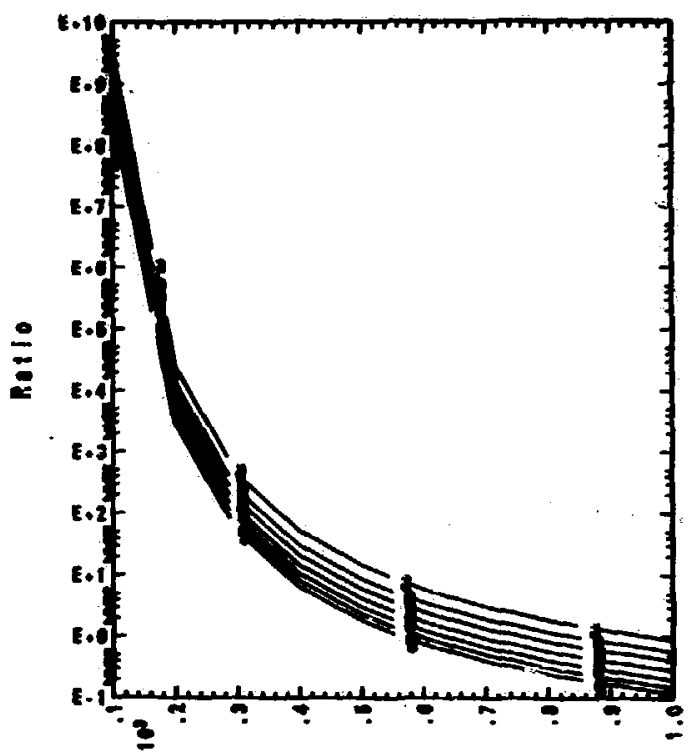

Electron Temerature |ov|
Treo. 2.

52- 5.000--03 Ze- 4.0000-01 Tr=0.

(ii) = (botps, hets)

(D) = (hys, hyi)

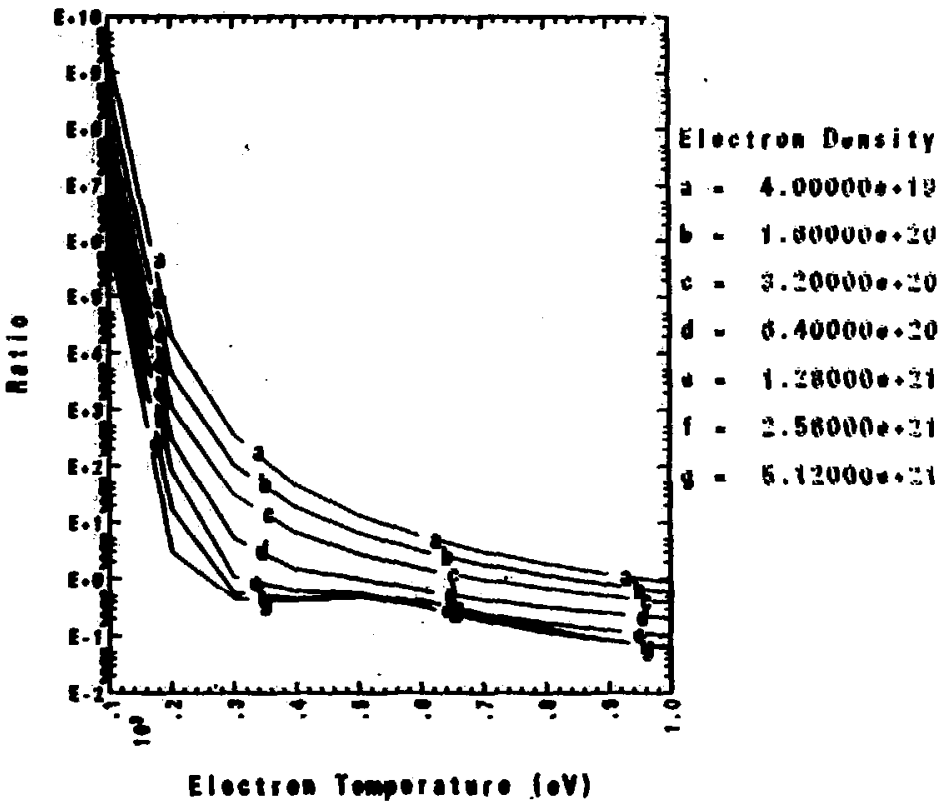


2. $1352=1.0000-00 \quad 20=0$.

(ii) - (byt,hyi)

(b) - (toud, hosps)

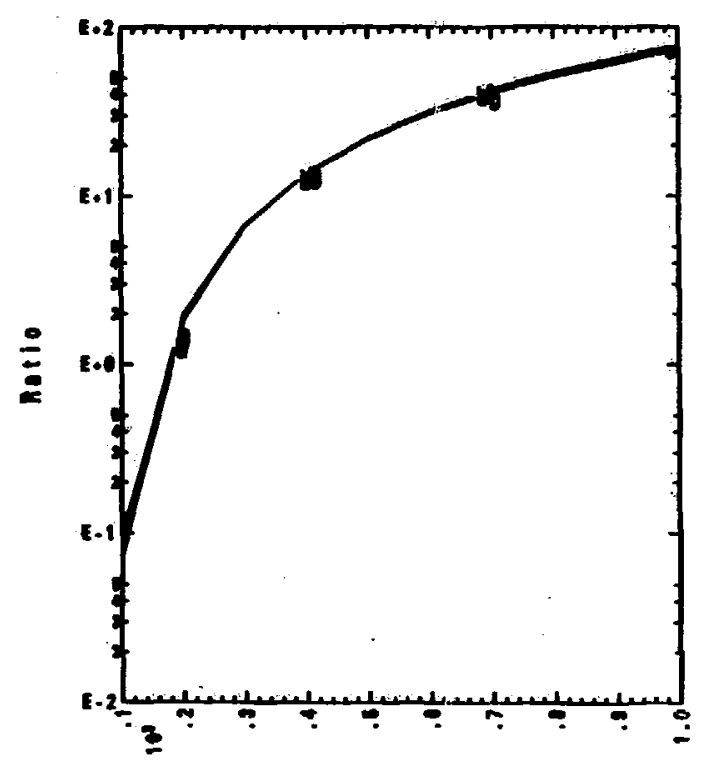

Elogtron Tomperature (oV)

Tre 0.
2. 13

$82=5.0000-03$

2

20- 4.0000-01 Tr=0.

(II)-(hy2,kyl)

(0) = (heat, ho2ps)

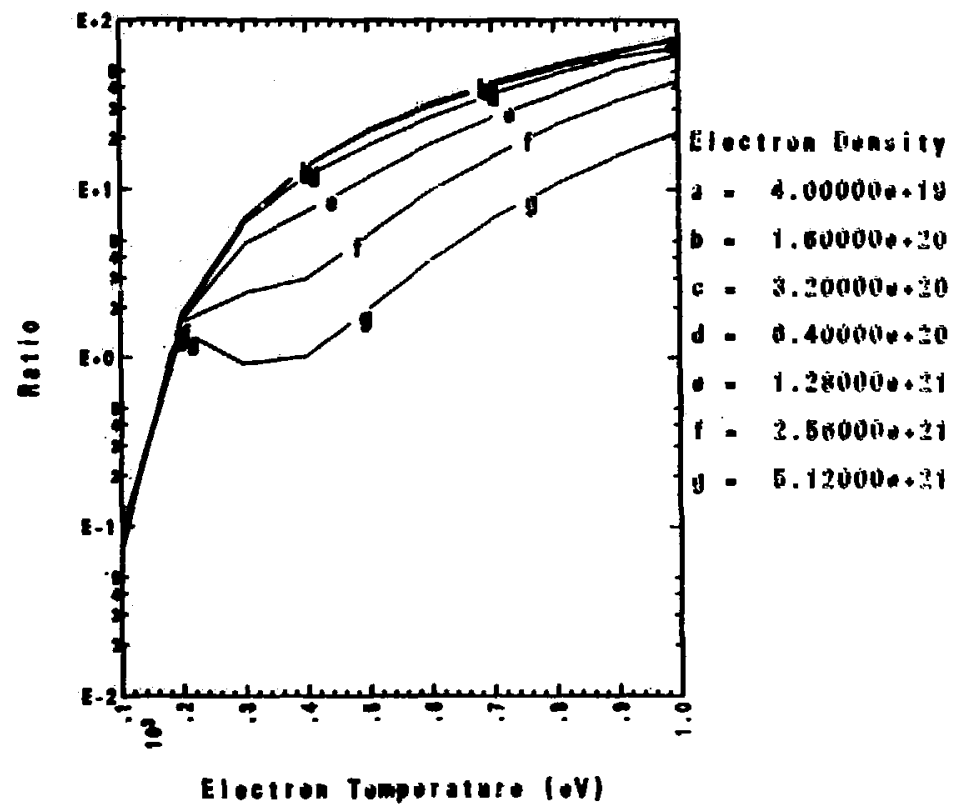



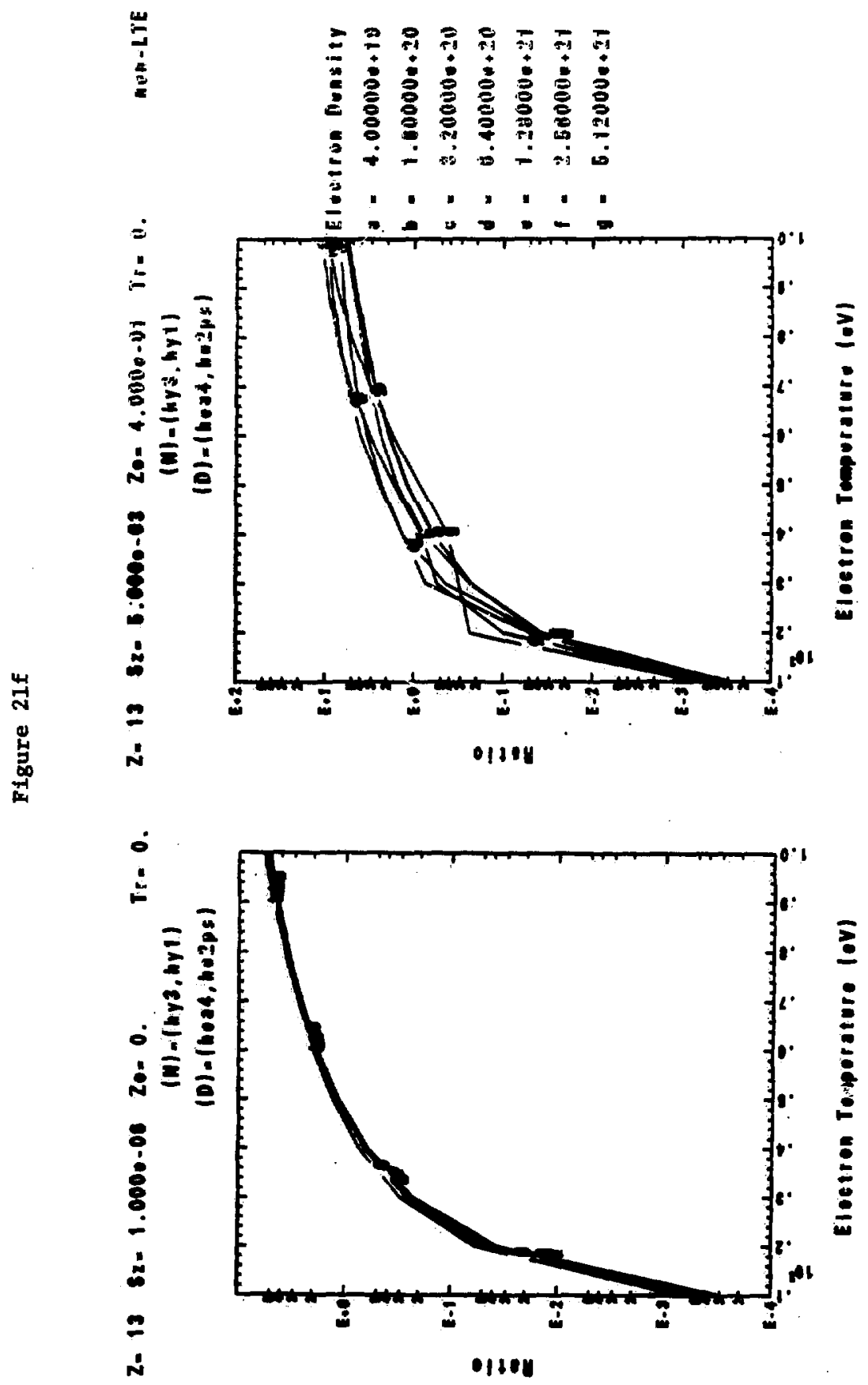
Figure 218
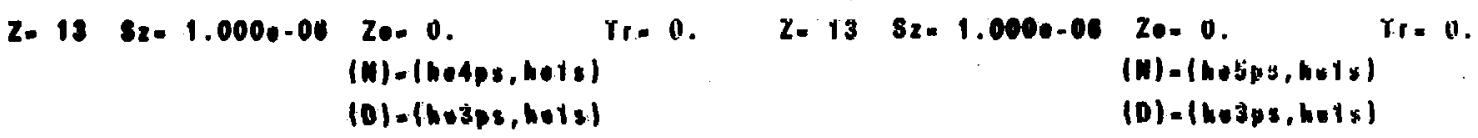

Aug-LTE
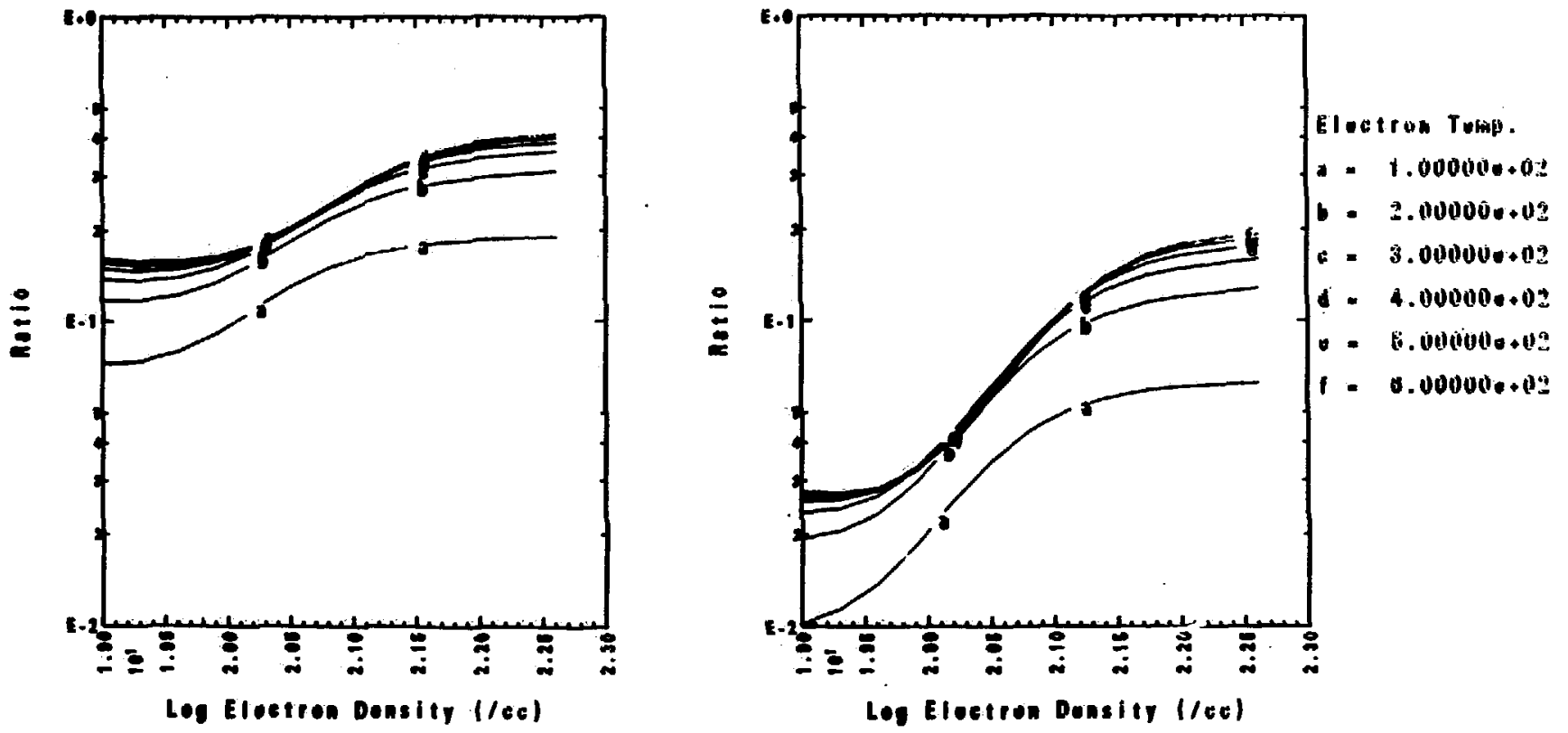

1 
electron density; b) the He-like $\left[1 s^{2}\left({ }^{1} S_{0}\right)-1 s 3 p\left({ }^{1} P_{1}\right)\right]$ "Hep" resonance line to He-like $\left[1 s^{2}\left({ }^{1} S_{0}\right)-1 s 2 p\left({ }^{3} P_{1}\right)\right]$ "IC" intercombination line ratio versus the electron density; c) the ratio of "He ${ }_{\alpha}$ " to ["IC" + "mn" + "st"] versus the electron density where "mn" and "st" are the $\left[1 s^{2} 2 p\left({ }^{2} P_{3 / 2,1 / 2}\right)-1 s^{2} p^{2}\left(2 S_{1 / 2}\right)\right]$ and $\left[1 s^{2} 2 s\left(2 S_{1 / 2}\right)-\right.$ $1 s 2 s(3 \mathrm{P}) \cdot 2 \mathrm{p}\left(\mathrm{P}_{3 / 2,1 / 2)}\right) \mathrm{Li}-$-like dielectronic satellite line intensities, respectively; d) the He-lite $\left[1 s^{2}\left({ }^{1} S_{0}\right)-1 s 3 p\left({ }^{1} P_{1}\right)\right]$ "Hes" to H-like (1s-3p) "Ly ${ }_{\beta}$ " resonance line ratio versus the electron temperature; e) the H-like (1s-2p) "Ly $y_{\alpha}$ resonance to He-like $\left[1 / 2 p\left({ }^{1} P_{1}\right)-2 p^{2}\left({ }^{1} D_{2}\right)\right]$ dielectronic satellite line ntio versus the electron temperature; $f)$ the "Lyק" to He-like $\left[1 s^{2} p\left({ }^{1} P_{1}\right)-2 p^{2}\left({ }^{1} D_{2}\right)\right]$ dielectronic satellite line ratio versus the electron temperature; and g) the electron density versus the high-n He-like resonance line rntios $\left[1 s^{2}\left({ }^{1} S_{0}\right)-1 s 4 p\left({ }^{1} P_{1}\right)\right] ~ " H e \gamma " ~ t o ~\left[1 s^{2}\left({ }^{1} S_{0}\right)-\right.$

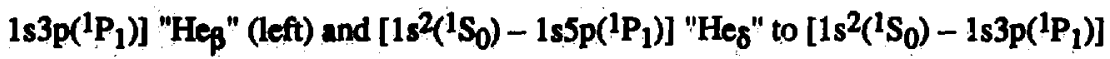
"Hep" (right). These "RATION" (CRE) predictions are used in discussed in further detail in Chapter 4 and used in Chapters 5 and 6 to analyzed the experimentally measured line intensity ratios.

\subsection{Radiation Trapping Effects}

The issue of radiation trapping due to the plasma opacity must be considered in both the theoretical modeling of these high energy plasmas as well in the design and execution of experiments to measure the spectral emission features. Radiation emitted from a volume of plasma may be absorbed (and possibly reemitted in $4 \pi$ steradians) or scattered before it escapes from the medium. 
Radiation trapping has a number of effects on the emitted spectral radiation. First, if the plasma is optically thick along the line of sight of the observer (spectrometer), the measured spectral line intensity represents an attenuated signal. Therefore, characterization of the plasma (such as determinations of the local plasma electron temperature or density, etc.) based on spectroscopic measurements of an optically thick plasma will likely be in error. Secondly, the extent of this reabsorption is dependent on the plasma path length the radiation must traverse in escaping the plasma volume. This becomes a significant problem if the plasm is optically thin along a direction other than along the spectrometer line-of-aight because the angular distribution of the emitted radiation is modified by the shape of the plasma as well as gradients in the plasma. A more important effect is that reabsorption of the radiation within the plasme volume affects the overall population kinetics of the participating ion. This may lead to changes in th overall level populations and the charge state distribution of the plasma thus altering the emission spectra. Finally, opacity effects play a key role in modifying the spectral line shape of the emission line. This issue is especially important when using a laser-produced plasma as either a laser medium or as a flashlamp source for photopumping particular transitions.

The modeling of optically thin plasmas is already a highly complex procedure. Plaem opecity effects simply adds more uncertainty to these calculations. In fact, the proper treatment of the plasma opacity effects remains a highly complex subject which has not yet been fully accounted for in any detailed plasma or atomic model and a formal treatment of radiation trapping would be beyond the scope of this work. Instead, I present the simplest diecussion on line opacities and refer the reader to several detailed discussion on the subject by Kaplan and Pikelner (1970), McWhirter (1965), Mihalas (1978), Osterbrock (1974), and Rybicki and Lightman (1979).

Consider a particular photon emitted by the plasma. The extent of its reabsorption in the plasma depends directly on the optical path in which it must travel in order to 
escape. Trapped radiation has the greatest probability of escape through the "thinest" dimension of the plasma volume. And therefore, one must quantify this probability to escape from the plasma. The "optical depth", $\tau_{0}$, is often used in which an escape probability $\left[P_{e}=e^{-\tau_{0}}\right.$ ] car be calculated base on the central frequency of the particular line transition. This simple "escape probability" method is typically used to modify the radiative decay rates by acting as a rate multiplier such that $A_{\mathrm{j} 1} \rightarrow$ $A_{j 1} \cdot P_{e}$. This effectively attenuates the spectral line intensity along the line of sight of the spectrometer and also modifies the rate equations governing the population kinetics of ions in the plasma. The number of optical depths along a plasma path of length $L$ for a particular transition $\lambda_{\eta_{1}}$ can be estimated by multiplying the center-line absorption cross section (based on a doppler line profile) by the number of absorbers in that plasma volume traversed by the photon. This is expressed by the following formula of Osterbrock (1974):

$$
\sigma_{m b}(1 s \rightarrow n p)=\frac{3 \cdot \lambda_{n 1}^{3}}{8 \pi} \cdot\left(\frac{m_{H}}{2 \pi k T}\right)^{1 / 2} \cdot A_{n p, 1 s}
$$

where $\tau\left(v_{0}\right)=\left(n_{\text {brorbers }} L\right) \cdot \sigma_{\text {abs }}(1 s \rightarrow m)$ is the center line opacity for a plasma of size $L$.

The number of line optical depths in a plasma can be directly computed using the following formula:

$$
\tau=5.12 \times 10^{-34}:\left(n_{1} \cdot L\right) \cdot\left(\frac{g_{u}}{g_{1}}\right) \cdot\left(A_{u l} \cdot \lambda^{3}\right) \cdot\left[\frac{M_{i}}{k T_{i}(k e V)}\right]^{1 / 2},
$$

where a Doppler width has been assumed: $\frac{\Delta \lambda_{\mathrm{D}}}{\lambda}=2.44 \times 10^{-3} \cdot\left[\frac{\mathrm{M}_{\mathrm{i}}}{\mathrm{kT} \mathrm{T}_{\mathrm{i}}(\mathrm{keV})}\right]^{1 / 2}$,

$$
A_{\text {ul }}=\text { the radiative decay rate }\left(\sec ^{-1}\right), \lambda=\text { wavelength in }(A), M_{i}=\text { the nuclear }
$$


mass in (A.U.), $\mathrm{g}_{\mathrm{g}} / \mathrm{g}_{1}=$ the ratio of statistical weights, $\mathrm{n}_{\mathrm{i}}=$ the number density of absorbing (lower level) ions in $\left(\mathrm{cm}^{-3}\right)$, and $\mathrm{L}=$ the plasma size along the line of sight in (cm).

The number of optical depths (at center frequency based on a doppler line width) can be readily estimated using this formula. For instance, assuming a plasma size of $50 \mu \mathrm{m}$ (along the line of sight) and a temperature of $400 \mathrm{eV}$, the number of optical depths for an aluminum plasma is:

$\begin{array}{lll} & \text { Heac }\left(1 \mathrm{~s}^{2}=1 \mathrm{~s} 2 \mathrm{p}\right) & \text { Lyo }(1 \mathrm{~s}-2 \mathrm{p}) \\ \lambda(\AA) & 7.757 & 7.173 \\ A(\sec -1) & 2.93 \times 10^{13} & 1.78 \times 10^{13} \\ \tau_{0}\left(10^{21} \mathrm{~cm}-3\right) & \approx 78 \cdot f_{\mathrm{He}} & \approx 33 \cdot f_{\mathrm{H}} \\ \tau_{0}\left(10^{\left.2 \mathrm{em}^{-3}\right)}\right. & \approx 7.8 \cdot f_{\mathrm{He}} & \approx 3.3 \cdot f_{\mathrm{H}}\end{array}$

where $f_{\mathrm{H}}$ and $f_{\mathrm{He}}$ are the fraction of ions in the ground states of the $\mathrm{H}$ and $\mathrm{He}$ charge states, respectively.

This is only a rough estimate. We see that the "He ${ }_{\alpha}$ " and "Ly $y_{\alpha}$ " lines will have their highest line opacities at low temperatures and high densities. The ramifications of this problem are presented in Chapter 5 where the aluminum $\mathbf{K}$-shell data is analyzed. Similar estimates for the line opacity are used in the "RATION" atomic models described earlier in this section and agree within a factor of two or better. The calculated optical depths from "RATTON" have been plotted in Figure 22 for a range of electron temperatures and densities encountered during these experiments. It should be noted that since the line opacity is based roughly on the number of lower level absorbers, the charge state distribution and level population kinetics are very important factors affecting the predicted CRE opacities.

This is only a very simple treatment of plasma opacity effects based on the 
Figure 22. The optical depths of various $\mathrm{H}$-like and He-like $\mathrm{Al}$ resonance and dielectronic satellite lines have been computed using t ne "RATION" (CRE) model are shown for a range of electron temperatures and densities. A $50 \mu \mathrm{m}$ plasma size was used for these calculations. (Note: "a" and " $b$ " correspond to the "1s-2p" and "1s-3p" transitions, respectively). 


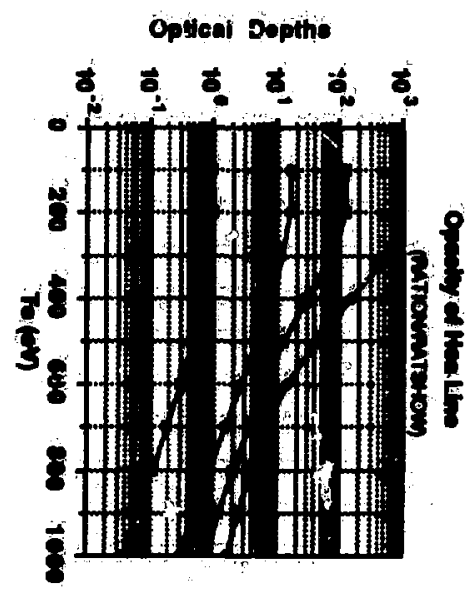

optiod Doptise

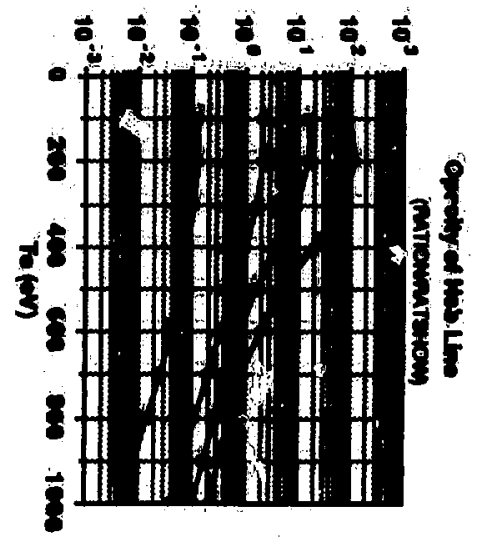

Opukal Dopine

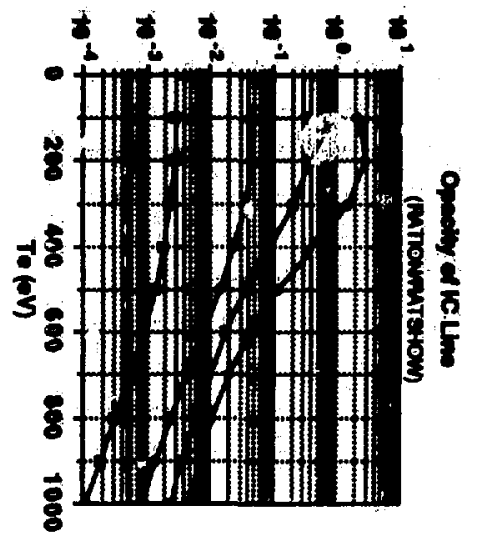

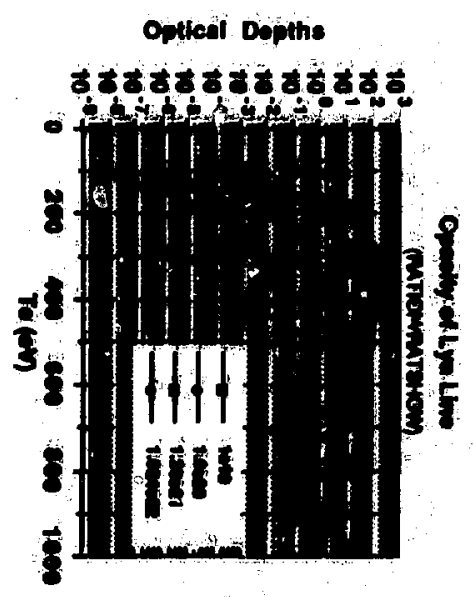
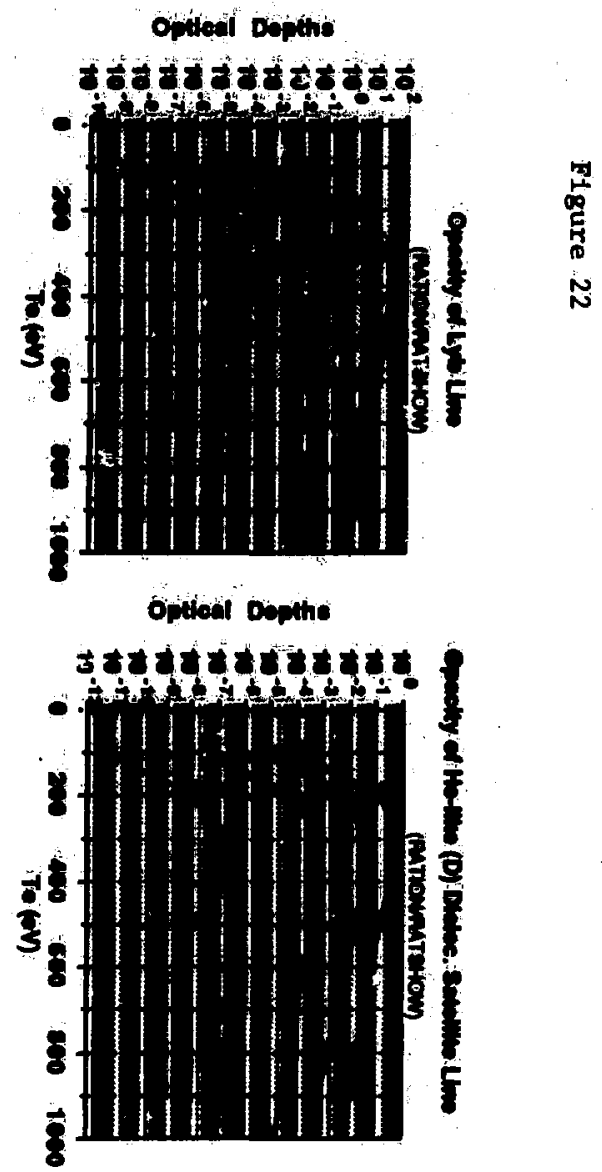
assumption that the plasma in thinest (in opacity) along the line-of-sight of the spectrometer and that the plasma is isotropic. A much more elaborate treatment is sometimes applied in which optical depths based on the various gradient scale lengths in the plasma are calculated and used. For instance, since the optical depth is proportional to the number of absorbers in the plasma volume, it is also directly related to the electron density in the plasma. Therefore, a general estimate of the opacity along the axis of a microdot plasma can be made bused on the density scale leagth in that direction. Similarly, rough estimates of the plasma opacity can be made based on temperature gradient scale lengths. In general, the density scale lengths along the axis of the microdot plasmas in this work are larger than the $50 \mu \mathrm{m}$ radius of the targets and the plasma has the smallest opscity along the line of sight of the spectrometers in these experiments.

\subsection{Important Plasma Parameters}

There are a few important hydrodynamics quantities characteristic of these laser-produced plasmas which should be considered when modeling or observing the plasmas experimentally. The hydrodynamics of this laser-plasma is a highly complex and intense reseurch area and a formal discussion on radiation transport theory and hydrodynamics of the plasma is beyond the scope of this thesis. Two excellent references on the subject are by C. Max (1981) and by R. Fabbro et al. (1985). I will just review some hydrodynamic quantities that havs been considered during the execution and analysis of these experiments using very simple empirical formulae from C. Max (1981) and L. Spitzer (1962). For discussion purposes only in this section, I cite values for important hydrodynamic quantities based on the following plasma parameters (unless otherwise specified) for a typical aluminum plesma: 


$$
\begin{aligned}
& Z=13, A=27 \text { A.U. } \\
& n_{e}=10^{21} \mathrm{~cm}^{-3}, T_{i}=T_{e}=500 \mathrm{eV} \\
& I_{a b s}=10^{14} \mathrm{~W} / \mathrm{cm}^{2}, \lambda_{L}=0.526 \mu \mathrm{m}, \tau_{L}=1.0 \mathrm{~ns} .
\end{aligned}
$$

I will first consider what the pertinent time and plasma scale lengths are in these microdot plasmas. These directly affect the temporal and spatial resolution required to study these plasmas. Are the radiative lifetimes and collision times short enough so that the atomic processes ongoing in these laser-produced plasmas are roughly in steady state so that detailed balance approximations can be made? How does this frame time relate to various equilibration times in the plasma? The relevant time for these plasmas is the time in which an ion (being studied) sits in a plasma region that is at relatively constant density and temperature, or else in the spatially resolved volume ( $\Delta x=50 \mu \mathrm{m}$ for the FCXS) element of plasma. The plasma (ion) velocity is

of order $v_{\mathrm{ion}} \approx 10^{7} \mathrm{~cm} / \mathrm{sec}$. Thus, the time in which an ion is within the $50 \mu \mathrm{m}$

FCXS resolution element is $\tau=\Delta \times / v_{\text {ica }}=\left(5 \times 10^{-3} \mathrm{~cm}\right) /\left(\approx 10^{7} \mathrm{~cm} / \mathrm{sec}\right) \approx 50 \mathrm{ps}$. Ideally, the time resclution should be of order $50 \mathrm{ps}$. Unfortunately, this was possible for only the XSCS instrument ( $\approx 30$ ps resolution) and a time resolution of 250 ps was used for the FCXS instrument. Still, this 250 ps FCXS time resolution is smaller than the nominal CHROMA laser heating pulsewidth of $1.0 \mathrm{~ns}$.

The time required for the distribution of kinetic energies of identical particles to approach a Maxwellian distribution is often called the self-collision time, $t_{c}$, and is determined almost entirely by the rate of elastic collisions among these particles. This self collision time can be computed using an expression of Spitzer (1962): 


$$
t_{\text {coll }}=\frac{m^{1 / 2} \cdot\left(3 k T_{e}\right)^{3 / 2}}{5.71 \pi \cdot n_{e} \cdot e^{4} \cdot Z^{4} \cdot \ln \Lambda}[\mathrm{sec}]
$$

or for electrons,

$$
t_{e}=\frac{0.266 \cdot\left(T_{e}\right)^{3 / 2}}{n_{e} \cdot \ln \Lambda}[\mathrm{sec}]
$$

where $m$ is the electron mass, $T_{e}$ is the temperature $\left({ }^{\circ} \mathrm{K}\right)$ of the electrons, $n_{e}$ is the electron density $\left(\mathrm{cm}^{-3}\right), e$ is the electronic charge, $Z$ is the ionic charge and $\ln \Lambda$ is a slowly varying quantity tabulated by Spitzer and approximately equal to 10 for the plasma conditions typical to these laser-produced plasmas. This collision time is more readily estimated using the formula for electrons and ions [NRL Plasma Formulary, 1980]:

$$
\begin{aligned}
& v_{e}=2.91 \times 10^{-6} \cdot n_{e} \cdot \ln \Lambda \cdot T_{e}-3 / 2=2.6 \times 10^{12} \sec ^{-1} \text { or } \tau_{e} \approx 0.384 \mathrm{ps}, \\
& v_{i}=4.78 \times 10^{-8} \cdot n_{i} Z^{2} \cdot \ln \Lambda \cdot T_{i}^{-3 / 2}=3.08 \times 10^{11} \sec ^{-1} \text { or } \tau_{i}=3.25 \mathrm{ps},
\end{aligned}
$$

A more general formula is given by Spitzer for the equipartition time for test particles in a field of particles of another type as:

$$
t_{\mathrm{eq}}=\frac{3 \cdot \mathrm{m}_{1} \cdot \mathrm{m}_{2}}{8 \cdot(2 \pi)^{1 / 2} \cdot \mathrm{n}_{2} \cdot \mathrm{z}_{1}^{2} \cdot \mathrm{z}_{2}^{2} \cdot \mathrm{e}^{4} \cdot \ln \Lambda} \cdot\left[\frac{k T_{1}}{\mathrm{~m}_{1}}+\frac{k T_{2}}{\mathrm{~m}_{2}}\right]^{3 / 2}[\mathrm{sec}]
$$

where foc electrons in $a$ field of ions of charge $Z_{i o n}$ and mass $A_{i o n} \cdot M_{p}$

$$
t_{\text {eq }}(e-i o n)=\frac{252 \cdot A_{\text {ion }}}{n_{\text {ion }} \cdot Z_{\text {ion }}^{2} \cdot \ln \Lambda} \cdot\left[T_{e}+\frac{T_{\text {ion }}}{1836 \cdot A_{\text {ion }}}\right]^{3 / 2}[\text { sec] }
$$

An approximate formula for the electron-ion collision rate for laser-plasmas is from Attwood (1978): 


$$
v_{\mathrm{ei}}=6.1 \times 10^{12} \cdot\left[\left(\frac{\mathrm{z}}{10}\right) \cdot\left(\frac{1 \mathrm{keV}}{\mathrm{kT}_{e}}\right)^{3 / 2} \cdot\left(\frac{\mathrm{n}_{e}}{10^{21} \mathrm{~cm}^{-3}}\right)\right] \mathrm{sec}^{-1}
$$

For the typical aluminum plasma here, $v_{\mathrm{ei}}=2.24 \times 10^{13} \mathrm{sec}^{-1}$ which corresponds to a collision time of $4.5 \times 10^{-2}$ picoseconds and is much smaller than experimental sampling times. The fastest radiative decay rates for these plasmas are typically of order $10^{12}$ to $10^{14} \mathrm{sec}^{-1}$ corresponding to lifetimes of 1.0 to $0.01 \mathrm{ps}$. All of these characteristic times are much shorter than either the 30 or 250 ps sampling times of the XSCS and FCXS time resolving diagnostics, respectively. Therefore, the time resolved spectra sumples a time interval in which the atomic processes governing the population kinetics in the plasm should be in quasi-steady state. However, this does not mean that the charge state distribution will be in equilibrium since the typical ionization times are of the order of a few $100 \mathrm{ps}$. [This is besed on typical collisional ionization rates of $=10^{9} \mathrm{sec}^{-1}$.] This is of great importance and it implications will be discussed in further detril in Chapters 5 and 6.

There are several important plasma scale lengths that must be considered. The electron Debye length which is typically much smaller than the laser wavelength in the plasmas. The Debye length for these experiments is very small typically $\lambda_{D}=5$ $\times 10^{-3} \mu \mathrm{m}$ estimated using the expression:

$$
\dot{\lambda}_{\mathrm{D}} \equiv\left(\mathrm{T}_{\mathrm{d}} / 4 \pi \mathrm{n}_{\mathrm{e}} \mathrm{e}^{2}\right)^{1 / 2}=7.4 \times 10^{-7} \cdot\left[\left(\frac{T_{e}}{1 \mathrm{keV}}\right) \cdot\left(\frac{10^{21} \mathrm{~cm}^{-3}}{\mathrm{n}_{\mathrm{e}}}\right)\right]^{1 / 2}
$$

and the number of particles in a Debye sphere is:

$$
N_{D}=\frac{4 \pi}{3} n_{e} \lambda_{D}^{3}=1.7 \times 10^{3}\left[\left(\frac{T_{e}}{1 \mathrm{keV}}\right)^{3 / 2} \cdot\left(\frac{10^{21} \mathrm{~cm}^{-3}}{n_{e}}\right)^{1 / 2}\right]
$$

For the typical plasma conditions, $N_{D}=600$. Standard kinetic theory assumes that 
ND * 1 and that the typical scale lengths for the temperature, density, and velocity gradients be large compared to the electron-ion mean free path:

$$
\lambda_{\mathrm{mfp}}=30 \mu \mathrm{m} \cdot\left[\left(\frac{\mathrm{T}_{\mathrm{e}}}{1 \mathrm{keV}}\right)^{2} \cdot\left(\frac{10^{21} \mathrm{~cm}^{-3}}{\mathrm{n}_{e}}\right) \cdot\left(\frac{10}{\ln \Lambda}\right)\right]
$$

This formula provides a rough estimate of the electron-ion mean-free-path which

corresponds to $\lambda_{\mathrm{mfp}}=7.5 \mu \mathrm{m}$ for the typical aluminum plasma conditions characteristic of these experiments. This is approximately a factor of 10 smaller than the measured scale lengths in the plasma.

When a high powered laser beam illuminates a solid target, a hot dense plasma is created at the surface of the target which "blows off" away from the target surface. Thermal energy is deposited in the initially solid turget, then flows towards cold, high density regions of the target. This plasma rapidly flows away from the target surface towards the laser beam with an outward flow of kinetic energy from dense to less dense regions of the evolving plasme plume. The blow-off velocity of the plasma is roughly equal to the local sound speed at the critical density:

$$
c_{s}=\sqrt{\mathrm{ZT}_{A} \mathrm{M}_{\mathrm{i}}}=3 \times 10^{7} \cdot\left[\left(\frac{\mathrm{Z}}{\mathrm{A}}\right)^{1 / 2} \cdot\left(\frac{\mathrm{T}_{e}}{1 \mathrm{keV}}\right)^{1 / 2}\right] \frac{\mathrm{cm}}{\mathrm{sec}} .
$$

For the typical aluminum dot plasmas in these experiments, we find this blow-off vilocity of approximately $3 \times 10^{7} \mathrm{~cm} / \mathrm{sec}$. This is well within typical values of $10^{7}$ and $10^{8} \mathrm{~cm} / \mathrm{sec}$ quoted by other researchers. The propagation of the laser light from low to higher density regions is modified by the free electrons generated by the ionizing plasma and a dispersion relationship can be written for the laser light: 


$$
\frac{\omega_{p}^{2}}{\omega_{L}^{2}}=1-\frac{k_{L}^{2} \cdot c^{2}}{\omega_{L}^{2}}, \text { where } \omega_{p}=\left[\frac{4 \pi e^{2} n_{e}}{m_{e}}\right]^{1 / 2}
$$

and $\omega_{\mathrm{L}}$ and $\mathrm{k}_{\mathrm{L}}$ are the frequency and viavenumber of the laser. At the critical density,

$$
\mathrm{n}_{\text {crit. }}=1.1 \times 10^{21} \cdot\left[\frac{1 \mu \mathrm{m}}{\lambda_{L}}\right]^{2} \mathrm{~cm}^{-3} \text {. }
$$

the plasma becomes opaque to the laser light and the light no longer propagates into the plasma. For the $0.526 \mu \mathrm{m}$ light used in these experiments, the critical density is approximately $4.4 \times 10^{21} \mathrm{~cm}^{-3}$.

The classical picture of the interaction of the laser beam with this plasma is that all of the laser absorption processes occur in the underdense region, $n_{e}<n_{\text {crit, }}$, of the plasma plume. Cold, high density regions are heated by electron thermal conduction. The overall hydrodynamics of the evolving plasma is highly complex and very large computer simulations are used to study these laser-produced plasmas. I will compare some of the new data obtained in this work with predictions of 2 dimensional "LASNEX" hydrodynamics simulations in Chapter 5.

One of the key elements of these experiments was the goal of measuring the $x$-ray emission from ideally isotropic (isodense and isothermal) plasma volumes. The new simultaneously space and time resolving instruments (FCXS and XSCS) allowed for spatial imaging in one dimension, along the plasma and laser axis. The remaining dimensions, representing the cross section of the plasma plume, had to be controlled by the design of the microdot targets and the expected hydrodynamics of the plasma plume that was formed. The density and velocity gradient scale lengths are approximately determined by the hydrodynamics of the plasma flow. For very short heating laser pulses, the scale lengths are determined roughly as $c_{s} \cdot \tau_{L}$ where $c_{s}$ is 
the corcnal sound speed and $\tau_{L}$ is the laser pulse width. For longer pulses when $c_{s}$. $\tau_{L}$ becomes large compared to the effective focal spot size, the scale lengths are determined by the effective geometrical divergence of the plasma flow. This is graphically shown in Figure 23 for the two cases. [C. Max (1C81)] In general for planar targets, the plasma blow-off can be expected to be planar if

$$
R_{\text {focal spot }}>150 \mu \mathrm{m} \cdot\left[\left(\frac{Z}{A}\right)^{1 / 2} \cdot\left(\frac{T_{0}}{1 \mathrm{keV}}\right)^{1 / 2} \cdot\left(\frac{\tau_{L}}{1 \mathrm{~ns}}\right)\right]
$$

We find that for the typical aluminum plasma parameters, this quantity is approximately $75 \mu \mathrm{m}$ which is smaller than the $R_{\text {focel }} \approx 125 \mu \mathrm{m}$ focal spot size (radius) used in these experiments. The actual isodensity contours (measured using holographic interferometry) typical for these microdot plasmas fall somewhere in between these two cases. The density across the center region where the actual 100 $\mu \mathrm{m}$ diameter aluminum microdot target is located should correspond to a fairly isodense profile perhaps varying by $\pm 10 \%$.

The electron temperature in the corona region of the plasma tends to be moderated by thermal conduction and is fairly uniform in space. The electron temperature scale lengths, $L_{T}=T_{e} \cdot\left|d T_{\alpha} / d x\right|^{-1}$, are typically very large in the underdense region. Typically, the ion temperature gradients are of little importance relative to electron gradients since the ion thermal conduction is typically small and electron-ion coupling negligible. Also, the ion temperatures are usually much lower than electron temperatures. Often, an isothermal approximation is used in which the temperature of the plasma is assumed to be $a$ constant. This is valid if the outwand heat flux from the critical surface is large enough to balance the cooling in the underdense region 
Figure 23. The geometrical divergence of the plasma flow and plasma scale lengths for a planar microdot target can be predicted using a simple empirical formula based on the laser irradiance conditions. [Max et al. (1981)] 


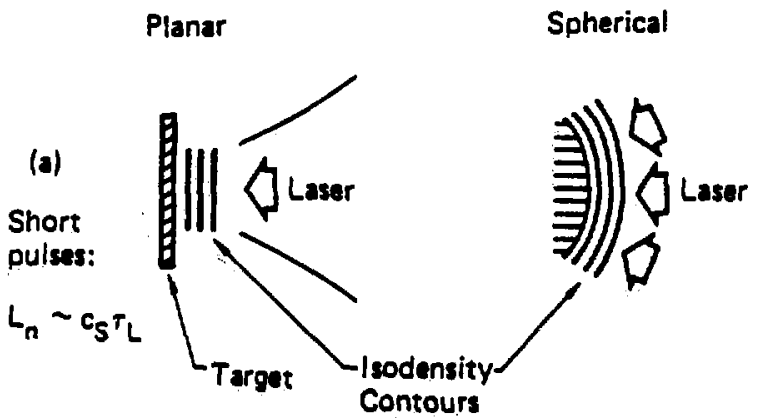

Isodensity contours typical of short-pulse experimeats. The density scale length $\mathrm{L}_{\mathrm{n}}$ is determined by the laser pulse length for both planar and spherical targets: $L_{n}=C_{g} T_{L}$.

Planar

(b)
Long
pulses:
$\sim R_{\text {eft }}$

$$
R_{\text {eff }} \sim 2 R_{\text {spot }}
$$

Spherical

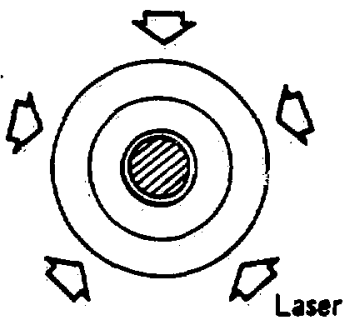

$\mathbf{R}_{\text {eft }} \sim \mathbf{R}_{\text {target }}$

Isodensity contours typical of long-pulse experiments. The density scale length is limited by the geometrical divergence of the plasma now. For planar targets the effective radius of curvarure $\mathbf{R}_{\mathbf{e f r}}$ is roughly twice the radius of the focal spot. 
due to plasma expansion. Then the rate of absorption of laser energy will be approximately equal to the rate of heat conduction near the critical density, $\mathrm{n}_{\text {crit: }}$ $I_{a b s}=f \cdot\left[T_{d} / m_{e}\right]^{1 / 2} \cdot\left(n_{c r i t} T_{e}\right)$. This can also be written as:

$$
\frac{T_{e}}{1 \mathrm{keV}}=0.6 \cdot\left[\frac{1}{f} \cdot\left(\frac{I_{a b s}}{10^{14} \mathrm{~W} / \mathrm{cm}^{2}}\right) \cdot\left(\frac{\lambda_{L}}{1 \mu \mathrm{m}}\right)^{2}\right]^{2 / 3}
$$

where $f$ is the "flux limiter" used in the heat transport models. For $f=0.03$ (typical value used to model planar plasmas) we get $T_{c}=2480 \mathrm{eV}$. For $f=0.4$ (value used in comparisons of "LASNEX" simulations with the new measurements in this study), we get $T_{e}=438 \mathrm{eV}$ which is very close to experimentally measured temperature values.

The isothermal plasma solution to the one dimensional hydrodynamic equations (continuity, momentum, and energy flow) has been found by a number of authors such as Fabbro et al. (1985). For a one dimensional isothermal plasma, the electron density falls off exponentially and can be written as,

$$
\frac{n_{e}}{n_{\text {crit. }}}=\beta \cdot \exp \left(-\frac{x}{L}\right) \text { for scale length } L \text {. }
$$

The "1/e" scale lengths using the interferometry density measurements and assuming an exponential electron distribution range from approximately 70-100 $\mu \mathrm{m}$ during the heating laser pulse to $120-150 \mu \mathrm{m}$ after the laser is turned off. Therefore, we see that the experimentally measured and theoretically predicted plasma scale lengths are in good agreement with each other and are typically just over $100 \mu \mathrm{m}$. This works out to be larger than the spatial resolution of the FCXS $(\sim 55 \mu \mathrm{m})$ and just larger than the coarse resolution of the XSCS $(=170 \mu \mathrm{m})$ spectrometers used in these experiments. 


\section{Chapter 4. Density Variation of Line Intensity Ratios in He-like and Ne-like Ions}

Line intensity ratios methods based on the $\mathrm{K}$-shell ( $\mathrm{H}$-like, He-like, and $\mathrm{Li}$-like ions) and L-shell (F-like, Ne-like, and Na-like ions) x-ray emission spectra are commonly used to characterize high-energy laboratory plasmas. The variations of these line intensity ratios with $T_{e}, n_{e}$, and ionization temperature provides important information of the atomic physics ongoing in these plasmas. Alternatively, these line ratios can be used to diagnose the conditions in the plasma provided that the excitation mechanisms are understood. In this chapter, I present the theory of three different line intensity ratios which are sensitive to variations in the electron density. The density dependence of these line ratios is typically due to collisional mixing processes which directly affect the excited (metastable) state populations. I examine the precise kinetic processes which govern the density dependence of each line intensity ratio. These ratios are: 1$)$ the often used He-like $\left[1 \mathrm{~s}^{2}\left({ }^{1} \mathrm{~S}_{0}\right)-1 \mathrm{~s} 2 \mathrm{p}\left({ }^{1} \mathrm{P}_{1}\right)\right]$ "He ${ }_{\alpha}$ " resonance line to the He-like $\left[1 s^{2}\left({ }^{1} S_{0}\right)-1 s 2 p\left({ }^{3} P_{1}\right)\right]$ "IC" intercombination

line ratio which is often referred to as " $\alpha$ "; 2) the ratio between high-n He-like resonance lines such as $\left[1 s^{2}\left({ }^{1} S_{0}\right)-1 s 4 p\left({ }^{1} P_{1}\right)\right] ~ " H e \gamma " ~ t o ~\left[1 s^{2}\left({ }^{1} S_{0}\right)-1 s^{3} p\left({ }^{1} P_{1}\right)\right]$ "Hep" resonance line ratio; and 3) the ratio between the Ne-like $\left.\left\{\left[1 s^{2} 2 s^{2} p^{6} 3 d(1 / 2,5 / 2) J=2\right]-\left[1 s^{2} 2 s^{2} 2 p^{6}(0,0)\right]=0\right]\right\}$ " $x^{\prime}$ electric quadrupole line and the $\left\{\left[1 s^{2} 2 s 2 p^{6} 3 p(1 / 2,3 / 2) J=1\right]-\left[1 s^{2} 2 s^{2} 2 p^{6}(0,0) J=0\right]\right\} " 3 A "$ resonance line. For the Ne-like levels, I will use the electron configuration (dropping the $1 \mathrm{~s}^{2}$ term from here on) along with the $\left(j_{1}, j_{2}\right)$ and $J_{\text {total }}$ for each level.

In section 4.1, I discuss the predicted density dependence of the " $\mathrm{He} \mathrm{\alpha}_{\alpha} \mathrm{IC}$ " - $\alpha$ " 
line intensity ratios as predicted by the standard collisional radiative equilibrium (CRE) analysis which is based on an equilibrium ionization distribution. In section 4.1.1, I present a discussion of the density dependence predicted by a detailed, quasi-steady state, collisional radiative K-shell model which allows for the examination of the level population kinetics without assuming an equilibrium (CRE) ionization distribution. Section 4.2 examines the high- $n \geq 3)$ resonance lines in He-like ions in a predominantly recombining plasma. Here, I show that recombination processes (mainly 3-body recombination) preferentially feed the high-n excited states. Since the radiative decay rates of the lower- $n$ levels tend to be higher than for the higher-n levels, a population inversion between the excited levels can develop. This phenomena would lead to inverted line intensity ratios in the high-n resonance lines. The quasi-steady state $\mathrm{K}$-shell model used in the present work, correctly accounts for these non-stationary processes including the collisional mixing between the excited states in the He-like system and predicis inverted high-n resonance line intensity ratios.

In section 4.3, I present a discussion of similar density sensitive metastable levels in Ne-like ions. A fairly simple, 37 level Ne-like, collisional radiative model is described in section 4.3.1. A detailed discussion of the predicted density dependence of the $\left.\left.\left[2 s 2 p^{6} 3 d(1 / 2,5 / 2)\right]=2\right],\left[2 s 2 p^{6} 3 p(1 / 2,3 / 2)\right]=1\right]$, and $\left[2 s^{2} 2 p^{5} 3 s(3 / 2,1 / 2) \mathrm{I}=1\right]$ levels in Ne-like $\mathrm{Mo}^{32+}$ and $\mathrm{Ag}^{37+}$ is presented in section 4.3.2. In section 4.3.3, a more detailed steady state, 89 level, collisional radiative Ne-like model is outlined. Both of these Ne-like models are used to interpret the data obtained in this work. These predicted density dependent line ratios are tested in Chapters 5 and 6 for high-energy, laser-produced K-shell and L-shell plasmas, respectively. 


\subsection{The Resonence to Intercombination Line Ratio: Density Variation of $n=2$ Lines in He-like Ions}

The electron density is most often derived from the He-like $1 s^{2}\left({ }^{1} S_{0}\right)-1 s 2 p\left({ }^{1} p_{1}\right)$ "He ${ }_{\alpha}$ " resonance line to the He-like $1 s^{2}\left({ }^{1} S_{0}\right)-1 s 2 p\left({ }^{3} P_{1}\right)$ "IC" intercombination line ratio commonly referred to as " $\alpha$ ". [Boiko et al. (197y; 1983); Skobelev et al. (1978); Gabriel and Jordan (1983); Kunze et al. (1968); Stavrakas et al. (1982); Vinogradov et al. (1975)] The density dependence of these line ratios is typically due to the interplay between collisional mixing processes fexcitations and deexcitations) and radiative decays which affect their excited level populations. In this section, I discuss the density dependence of the "He $/ \mathrm{IC}$ " line intensity ratio as predicted by standad collisional radiative equilibium CRE atomic theory as well as by non-stationary models which fully account for ionization and recombination processes from adjecent charge states.

The basic stricture of the lowest lying levels in a He-like ion is shown is Figure 24. These levels correspond to $n=2$ multiplet and are referred to as ${ }^{1} \mathbf{P}_{\mathbf{1}},{ }^{3} \mathbf{P}_{\mathbf{2}, 1,0}$, $1 \mathrm{~s} 2 \mathrm{~s}\left(1 \mathrm{~S}_{0}\right)$, and ${ }^{3} S_{1}$. Typically, all of these terms can be excited from the ground state with similar collisional excitation rates which may vary by factors of about 5-20. These levels decay via different radiative processes govemed by the usual multipole selection rules. The electric dipole-allowed radiative decay rates for the "He $e_{\alpha}$ and "IC" transitions are $A_{H e \alpha}=2.93 \times 10^{13} \sec ^{-1}$ and $A_{I C}=7.46 \times 10^{10}$

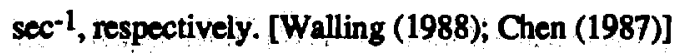

The ${ }^{1} P_{1}$ level decays to the ground level by the strong electric dipole allowed process and spectroscopically represents the " $\mathrm{He}_{\alpha}$ " resonance line. The transition 
Figure 24. The energy level diagram representing the lowest $n=2$ levels of the He-like ion. The $1 \mathrm{~s} 2 \mathrm{p}\left({ }^{3} \mathrm{P}_{1}\right)$ level decays to the ground state and corresponds to the "He $\alpha$ " resonance line and the $1 \mathrm{~s} 2 \mathrm{p}\left({ }^{3} \mathrm{P}_{1}\right)$ excited state level decays to the ground state as the intercombination " $\mathrm{C}$ " line. 

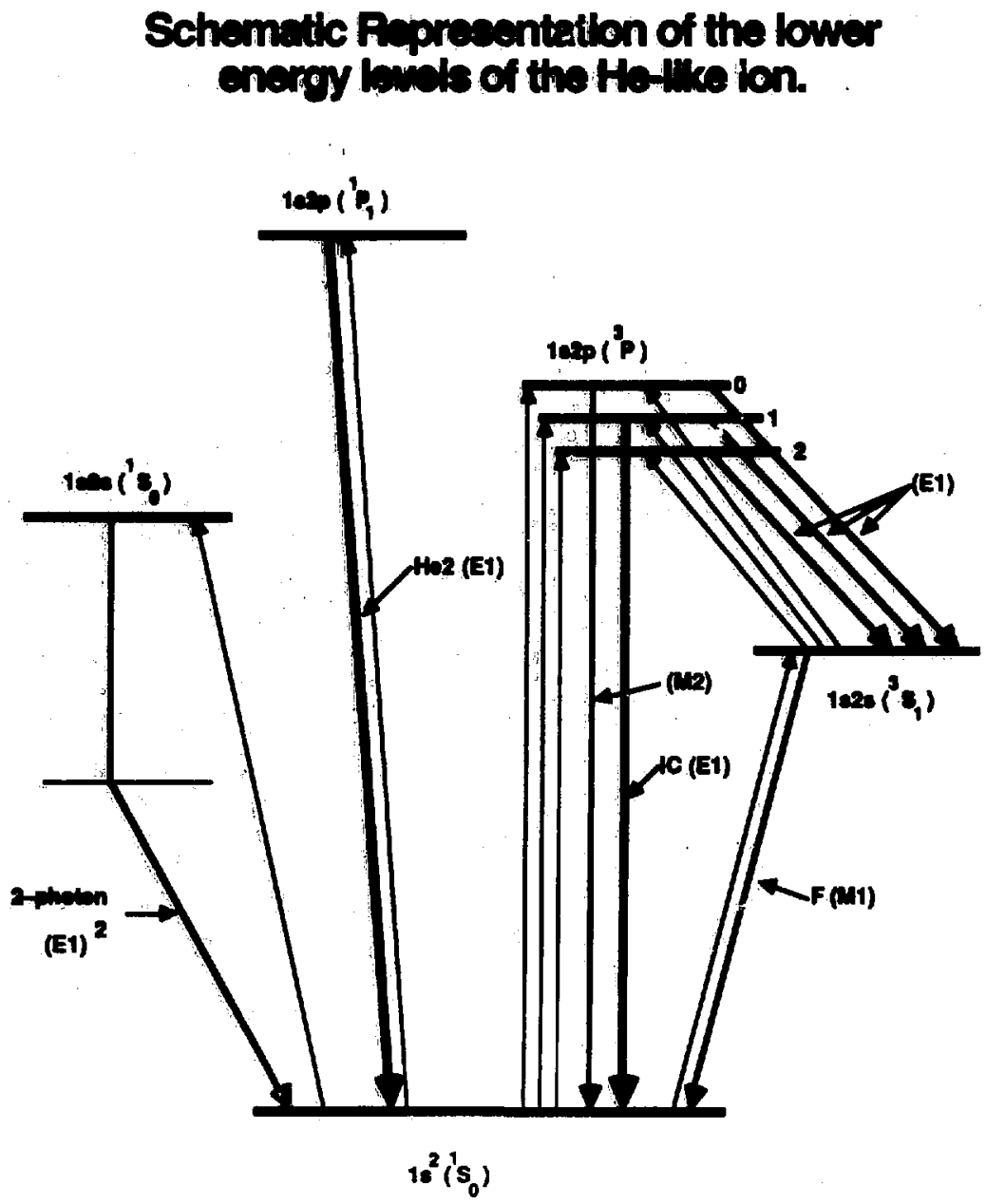

Figure 24 
rate to the $1 \mathrm{~s} 2 \mathrm{~s}\left({ }^{1} \mathrm{~S}_{0}\right)$ level is relatively small in comparison and is typically neglected. The ${ }^{3} \mathrm{P}_{1}$ level corresponds to the upper level of the "intercombination line" or "IC" and actually consists of a mixing of the three separate ${ }^{3} \mathrm{P}_{2},{ }^{3} \mathrm{P}_{1}$, and ${ }^{3} \mathrm{P}_{0}$ levels. As a simplification, this group will be considered as a single level, ${ }^{3} \mathbf{P}_{1}$. It should be noted that for the typical low-Z, high density laser plasmas, the intercombination line usually appears as a single spectroscopic feature and the individual transitions cannot be resolved. The term "intercombination" is due to the spin-orbit mixing with the ${ }^{1} P_{1}$ level and therefore allowing a transitions rate to the $1 \mathrm{~s}^{2}\left({ }^{1} \mathrm{~S}_{0}\right)$ ground state. There is a small transition rate to the ${ }^{3} \mathrm{~S}_{1}$ level which must also be accounted for. The $1 \mathrm{~s} 2 \mathrm{~s}\left({ }^{1} \mathrm{~S}_{0}\right)$ level decays to the ground level only via a two-photon decay resulting in a continuum rather than as a specific spectroscopic line and therefore is not readily observable. The ${ }^{3} S_{1}$ level docays by a magnetic dipole transition (often referred to as the "forbidden line" or "Ml") and is only observed in very low density plasmas.

The populations of these excited levels are also influenced by electron collisions which effectively compete with the radiative decay rates and transfer population between the levels. The density dependence of these He-like $n=1$ to $n=2$ transitions are illustrated in Figure 25 from Gabriel \& Jordan (1972) for Neon ( $Z=10)$ at a temperature of $300 \mathrm{eV}$. We can identify three separate density regions in which population is transferred from the metastable levels (those with relatively long radiative decay lifetimes) to the ${ }^{3} P_{1}$ and finaliy to the ${ }^{1} P_{1}$ level as follows:

1. For low densities, the ${ }^{3} S_{1}$ level population is transferred collisionally to the ${ }^{3} P_{1}$ level and therefore the "intercombination line" to "forbidden line" ratio increases with $\mathrm{n}_{\mathrm{e}}$.

2. For intermediate densities, all of the triplet population exists in the ${ }^{3} \mathrm{P}_{1}$ level. 
Figure 25. The density dependence of the various transitions in the $n=2$ level of He-like neon have been plotted as a function of the electron density. [Gabriel \& Jordan (1972)] These line intensity curves correspond to a temperature of $300 \mathrm{eV}$. The "11S-23S" transition represents the "forbidden" magnetic dipole line and the "1 1 -21S" transition represents the family of two-photon decays of the $1 \mathrm{~s} 2 \mathrm{~s}\left({ }^{1} \mathrm{~S}_{0}\right)$ level. Both of these lines are not normally observed from dense laser plasmas $n_{e} \geq$ $10^{19} \mathrm{~cm}^{-3}$ as indicative by the figure. The "1 $1 \mathrm{~S}-2^{3} \mathrm{P}$ " transition is the intercombination line "IC" while the " $1^{1} S-2^{1} P^{\prime}$ transition is the dipole allowed "He $e_{\alpha}$ transition. 
F1gure 25

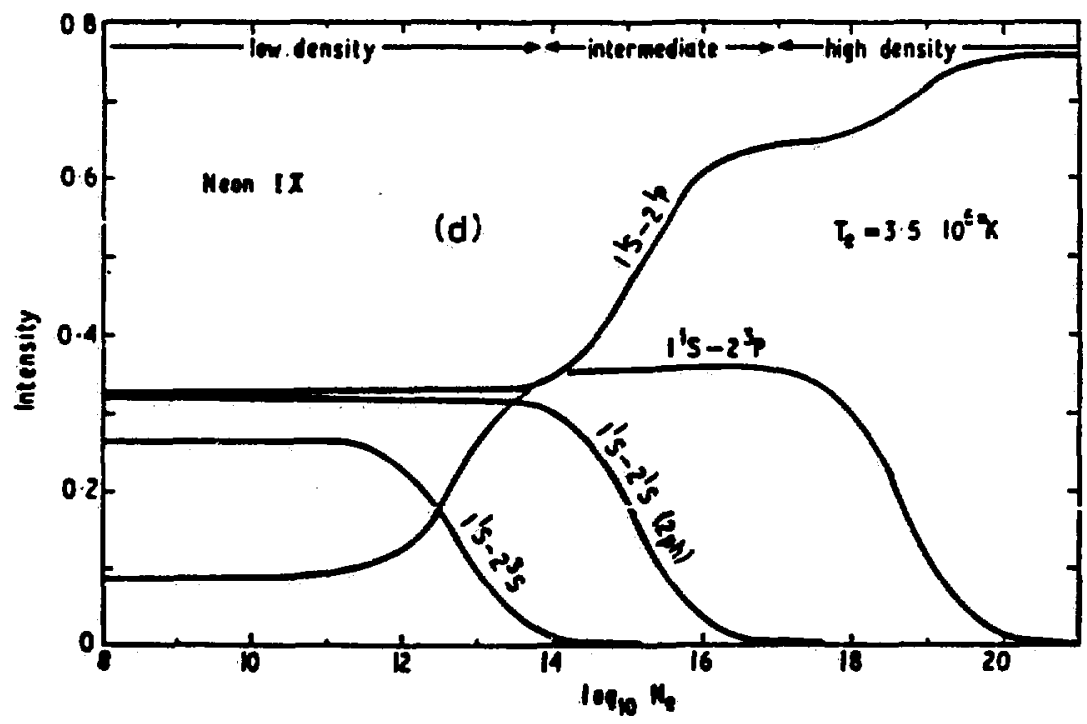


The $1 \mathrm{~s} 2 \mathrm{~s}\left({ }^{1} \mathrm{~S}_{0}\right)$ population is collisionally excited to the ${ }^{1} \mathrm{P}_{1}$ level. Therefore, the ${ }^{1} \mathrm{P}_{1}$ transition increases relative to both the "intercombination" and "2-photon" decay transitions as $n_{e}$ increases in this regime.

3. Finally, at very high densities typical of those in laser plasmas, the ${ }^{1} \mathbf{P}_{1}$ level population continues to grow at the expense of the other levels due to electron collisions. Consequently, the resonance line to "intercombination line" intensity ratio increases with $n_{e}$ until the density is so high that all excited electrons are collisionally transferred to the ${ }^{1} P_{1}$ level before any other radiative decays can take place and only the $\left[1 \mathrm{~s}^{2}\left({ }^{1} \mathrm{~S}_{0}\right)\right]-\left[1 \mathrm{~s} 2 \mathrm{p}\left({ }^{1} \mathrm{P}_{1}\right)\right]$ "He${ }_{\alpha}$ " line is observed. Actually, the ${ }^{1} \mathbf{P}_{1}$ level population always is low for an optically thin plasma due to the strong (dipole allowed) radiative decay to the ${ }^{1} S_{0}$ ground state.

This third (highest) density region is typical of laser-produced plasmas and in particular for the testing of the density dependence of the " $\alpha$ " line ratio. For He-like aluminum $(Z=13)$, this region corresponds to electron densities of $10^{19} \mathrm{~cm}^{-3}$ and higher. Ideally, the electron density can be determined by measuring the " $\mathrm{He}_{\alpha}$ " and "IC" intensities and comparing them to the theoretical density dependence of these lines.

The region in which these He-like aluminum lines exhibit this density dependence can be estimated by examining the functional forms of their line intensities. A rough CRE estimate of the line intensity can be made by assuming that the excited levels are populated primarily by collisional excitation from the ground state. The line intensity then becomes dependent on the ground state population $N_{g}$, the electron density $n_{e}$, the excitation rate between the ground state and the excited level $\langle v \sigma\rangle_{\mathrm{ij}}$, and the 
branching ratio for the radiative decay from that excited level back down to the ground state $\omega_{\mathrm{jj}}$ as shown in the following expression:

$$
I_{i j}=N_{j} \cdot A_{i j}=N_{i} \cdot n_{e}\langle v \sigma\rangle_{i j} \cdot \omega_{i j}, \text { where } \omega_{i j}=\frac{A_{i j}}{\sum_{k}\left(A_{k j}+n_{e}\langle v \sigma\rangle_{k j}\right)}
$$

The collisional transition rates between the $n=2$ excited levels can be obtained from a standard He-like aluminum model. For this analysis, I will use data provided by W. Goldstein and A. Osterheld (1988). The atomic data base is describe in more detail later in this chapter. The co'lisional excitation rates are computed using a multiconfigurational distorted wave (MCDW) calculation based on the Klapisch's parametric potential method [Klapisch $(1971 ; 1977)$ ] and are listed in Table 2. The "IC" line becomes sensitive to variations in the electron density when the collisional rate out of the ${ }^{3} \mathbf{P}_{1}$ level effectively competes with the radiative decay rate of the "IC" line or when $A_{I C}=n_{e}(c r i t) \cdot \Sigma<v \sigma>3 p$. The total collisional rate coefficient for transitions from the triplet level to the singlet level is $\Sigma<v O>3 \mathrm{P} \rightarrow 1 \mathrm{P}=1.55 \times 10^{-10}$ $\mathrm{cm}^{3} \cdot \mathrm{sec}^{-1}$. The radiative decay rates for the " $\mathrm{He}_{\alpha}$ " and "IC" lines are $\mathrm{A}_{\mathrm{He \alpha}}=2.93 \mathrm{x}$ $10^{13} \mathrm{sec}^{-1}$ and $A_{1 C}=7.46 \times 10^{10} \mathrm{sec}^{-1}$, respectively [Chen (1987)]. Therefore, $\mathrm{n}_{\mathrm{e}}$ (crit) $=4.8 \times 10^{20} \mathrm{~cm}^{-3}$. This is only a crude, estimate. The actual " $\mathrm{He} / \mathrm{IC}$ " line intensity ratio will be affected at lower densities since some of the ${ }^{3} \mathrm{P}_{1}$ population is collisionally transferred to the ${ }^{1} \mathrm{P}_{1}$ level at lower electron densities. A more sophisticated analysis can be done to fully study the density dependence of the "He $\mathrm{o}^{\mathrm{IC}} \mathrm{C}$ line ratio.

For the lower density regions $\left(\leq 10^{19} \mathrm{~cm}^{-3}\right)$, the $\Delta \mathrm{n}=0$ collision rates are too small 
Table 2. The collisional transition rate coefficients from the ground state to the $n=2$ He-like levels and the $\Delta n=0$ collisional rates between the $n=2$ levels are listed for an Al plasma at a temperature of $400 \mathrm{eV}$. 
Helium-like AI XII $n=2$ Collision Rate Coefficients

\begin{tabular}{l|c|c|c|c|c|c} 
& $1 \mathrm{~s} 2 \mathrm{~s}\left({ }^{3} \mathrm{~S}_{1}\right)$ & $1 \mathrm{~s} 2 \mathrm{p}\left({ }^{3} \mathrm{P}_{0}\right)$ & $1 \mathrm{~s} 2 \mathrm{p}\left({ }^{3} \mathrm{P}_{1}\right)$ & $1 \mathrm{~s} 2 \mathrm{p}\left({ }^{3} \mathrm{P}_{2}\right)$ & $1 \mathrm{~s} 2 \mathrm{~s}\left({ }^{1} \mathrm{~S}_{1}\right)$ & $1 \mathrm{~s} 2 \mathrm{p}\left({ }^{1} \mathrm{P}_{1}\right)$ \\
\hline $1 \mathrm{~s}^{2}\left({ }^{1} \mathrm{~S}_{0}\right)$ & $4.067 \mathrm{e}-14$ & $5.237 \mathrm{e}-14$ & $1.536 \mathrm{e}-13$ & $2.589 e-13$ & $1.367 \mathrm{e}-13$ & $9.680 e-13$ \\
$1 \mathrm{~s} 2 \mathrm{~s}\left({ }^{3} \mathrm{~S}_{1}\right)$ & - & $5.714 \mathrm{e}-10$ & $1.698 \mathrm{e}-09$ & $2.833 e-09$ & $1.434 e-11$ & $1.649 \mathrm{e}-11$ \\
$1 \mathrm{~s} 2 \mathrm{p}\left({ }^{3} \mathrm{P}_{0}\right)$ & & - & $8.132 \mathrm{e}-11$ & $1.076 \mathrm{e}-10$ & $4.388 \mathrm{e}-12$ & $8.382 \mathrm{e}-11$ \\
$\left.1 \mathrm{~s} 2 \mathrm{p}{ }^{3} \mathrm{P}_{1}\right)$ & & & - & $1.011 \mathrm{e}-10$ & $1.576 \mathrm{e}-11$ & $2.341 \mathrm{e}-11$ \\
$1 \mathrm{~s} 2 \mathrm{p}\left({ }^{3} \mathrm{P}_{2}\right)$ & & & & - & $4.395 \mathrm{e}-12$ & $4.777 \mathrm{e}-11$ \\
$1 \mathrm{~s} 2 \mathrm{~s}\left({ }^{1} \mathrm{~S}_{1}\right)$ & & & & & - & $5.728 \mathrm{e}-0 \mathrm{~S}$ \\
$1 \mathrm{~s} 2 \mathrm{p}\left({ }^{1} \mathrm{P}_{1}\right)$ & & & & & &
\end{tabular}

An electron temperature of $400 \mathrm{eV}$ has been assumed. The total collisional nues between these $\mathrm{n}=2$ kevels are: $\quad\left\langle 00>_{\text {botal }}\left({ }^{3} \mathrm{P}_{\mathrm{J}}-1 \mathrm{P}_{1}\right)=1.55 \times 10^{-10} \mathrm{~cm}^{3} \cdot \mathrm{sec}^{-1}\right.$, $<v 0>_{\text {total }}\left({ }^{3} \mathrm{P}_{\mathrm{J}}-3 \mathrm{~S}_{1}\right)=5.10 \times 10^{-9} \mathrm{~cm}^{3} \cdot \mathrm{sec}^{-1}$, $<00>_{\text {botal }}\left(3 \mathrm{P}_{\mathrm{J}}-1 \mathrm{~S}_{1}\right)=2.45 \times 10^{-11} \mathrm{~cm}^{3} \cdot \mathrm{sec}^{-1}$, $<v 0>_{\text {wotal }}\left({ }^{3} \mathrm{~S}_{1}-1 \mathrm{~S}_{1}\right)=1.43 \times 10^{-11} \mathrm{~cm}^{3} \cdot \mathrm{sec}^{-1}$, $<v>_{\text {wotal }}\left({ }^{3} \mathrm{~S}_{1}-1 \mathrm{P}_{1}\right)=1.65 \times 10^{-11} \mathrm{~cm}^{3} \cdot \mathrm{sec}^{-1}$, $<v 0>_{\text {wotal }}\left({ }^{1} \mathrm{~S}_{1}-1 \mathrm{P}_{1}\right)=5.73 \times 10^{-9} \mathrm{~cm}^{3} \cdot \mathrm{sec}^{-1}$. 
to effectively transfer population from the ${ }^{3} P_{1}$ and ${ }^{1} P_{1}$ levels and the ratio " $\alpha$ " is governed by the ratio of the total excitation rates feeding the triplet and singlet levels and is independent of the electron density. Here " $\alpha$ ", the "He$e_{\alpha} / \mathrm{IC}$ " line intensity ratio, is calculated to be:

$$
\alpha=\frac{N\left(2{ }^{1} P_{1}\right) \cdot A\left(2{ }^{1} P_{1} \rightarrow 1^{1} S_{0}\right)}{N\left(2{ }^{3} P_{1}\right) \cdot A\left(2^{3} P_{1} \rightarrow 1^{1} S_{0}\right)}
$$

A complete description of the predicted density dependence of the line ratio " $\alpha$ " can be found in a series of papers by Vinogradov et al. (1975), Skobelev et al. (1978), and Boiko et al. (1979; 1983). For high densities, " $\alpha$ " can be estimated using the following expression:

$$
\begin{aligned}
\alpha & =\frac{I_{\text {Hoa }}}{I_{I C}}=\frac{I\left(1 s 2 p^{1} P_{1} \rightarrow 1 s^{21} S_{0}\right)}{I\left(1 s 2 p^{3} P_{1} \rightarrow 1 s^{21} S_{0}\right)} \\
& =R+\frac{4 \cdot N_{e} \cdot\left[(R+1) \cdot C\left(2^{3} \rightarrow 2^{1}\right)+R \cdot C\left(2^{3} \rightarrow i\right)\right]}{A\left(1 s 2 p^{3} P_{1} \rightarrow 1 s^{21} S_{0}\right)}
\end{aligned}
$$

where, $\mathrm{C}\left(2^{3} \rightarrow \mathrm{i}\right)$ is the average rate coefficient for collisional ionization of the triplet levels, $C\left(2^{3} \rightarrow 2^{1}\right)$ is the collisional transition rate (averaged over the triplet levels) between the triplet and singlet states $1 \mathrm{~s} 2 l\left({ }^{3} \mathrm{~L}\right) \rightarrow 1 \mathrm{~s} 2 l$ ' ( $\left.{ }^{1} \mathrm{~L}\right)$, $A\left[1 s 2 p\left({ }^{3} P_{1}\right) \rightarrow 1 s^{2}\left({ }^{1} S_{0}\right)\right]$ is the radiative decay rate of the $1 \mathrm{~s} 2 \mathrm{p}\left({ }^{3} \mathrm{P}_{1}\right) \rightarrow 1 \mathrm{~s}^{2}\left({ }^{1} \mathrm{~S}_{0}\right)$ transition, and $R$ is the ratio of the rate coefficients for transitions from states with principal quantum number $n \neq 2$ to the $1 \mathrm{~s} 2 l\left({ }^{1} \mathrm{~L}\right)$ and $1 \mathrm{~s} 2 l$ ' ( $\left.{ }^{3} \mathrm{~L}\right)$ levels: 


$$
R=\frac{\left[C\left(1^{1} s_{0} \rightarrow 2^{1} s_{0}\right)+C\left(1^{1} s_{0} \rightarrow 2^{1} P_{1}\right)\right]}{\left[C\left(1^{1} s_{0} \rightarrow 2^{3} S_{1}\right)+\sum_{J=0}^{2} C\left(1^{1} S_{0} \rightarrow 2^{3} P_{J}\right)\right]}
$$

Here, a very simplified model has been used in which the levels $2{ }^{3} P_{J}$ and $23 S_{1}$ are assumed to be in a Boltzmann equilibrium and the following processes have been included: radiative decay of the $2{ }^{1} \mathrm{P}_{1}$ and $2{ }^{3} \mathrm{P}_{1}$ excited states, the collisional transitions $2^{1} \mathrm{~S}_{0} \rightarrow 2^{1} \mathrm{P}_{1}, 2^{3} \mathrm{~L} \rightarrow 2^{1} \mathrm{~L}$, and ionizution of the metastable triplet levels due to their longer radiative lifetimes. Also, the plasma is assumed to be optically thin for all radiative transitions.

Only transitions within the $n=2$ level have been accounted for in this simple model. The role of cascades from ( $n \geq 2)$ higher lying excited levels has been studied by Vinogradov et al. (1975). Vinogradov notod that cascade contributions to the singlet levels are not significant since the subsequent $n \geq 3$ singlet excited levels depopulate rapidly via radiative decays to the ground state. For high densities, the $n \geq 3$ triplet excited states provide an additional channel for collisional excitation of the $2{ }^{3} \mathrm{~S}_{1}$ and $2{ }^{3} \mathrm{P}_{\mathrm{J}}$ levels to these higher iying states. Electrons excited to the higher $\mathrm{n}$ (triplet) levels are subsequently ionized. Therefore, for these high densities, " $\alpha$ " is underestimated by the simple $n=2$ model. Vinogradov reports that contributions due to cascades from $n \geq 5$ levels are negligible and that cascades from the $(n=3)$ and ( $n=4)$ levels can be easily included into the model. I will show later in sections 4.1.1 and 4.2 a quasi-steady state K-shell model which fully accounts for both cascades and collisional mixing up to the $n=5$ level which demonstrates this point. [Goldstein et al. (1988)]

Contributions due to ionizations and recombinations from adjacent charge states have also been neglected in this simple model. These processes, and all the 
contributions from $n \geq 3$, are accounted for in a large atomic model such as "RATION" (described in Chapter 3) which assumes an equilibrium (CRE) charge state distribution. ["RATION" includes the high-n states as a single "lumped" term of the form (1snp).l The predicted CRE density dependence of " $\alpha$ " calculated using the "RATION" code was shown earlier in Figure 21a(left) for a range of electron temperatures between 100 and $1000 \mathrm{eV}$. The line ratio is fairly insensitive to the electron temperature except for the effect $T_{e}$ has on the overall CRE ionization distribution and for the small effects on the various recombination rates.

The " $\alpha$ " line ratio was also shown in Figure 21a(right) for an optically thick plasma using the escape factor probability correction of Cauble (1987) for the "RATION" (CRE) code. Here, a $50 \mu \mathrm{m}$ thick "alab" plasma has been assumed. For high densities (and also for low temperatures), the " $\mathrm{H} \varepsilon_{\alpha}$ " line intensity is highly optically thick and the " $\alpha$ "line ratio decreases as a function of density. In the regions in which the plasma is optically thick, the " $\alpha$ " line ratio becomes double valued so that for any particular value of " $\alpha$ ", there will be two corresponding densities. This further confuses the density dependence of the CRE " $\alpha$ " line ratio. The optically thick " $\alpha$ "line ratio can only be used if the one of the derived densities can be neglected. Furthermore, the role opacity plays in affecting the overall level population kinetics and the simple corrections made to treat opacity effects is at very best suspect as discussed earlier in Chapter 3. Clearly, optically thin piasmas should be studied first and well characterized before the more complex opacity and radiative transfer effects are included.

In Chapter 5, I compare experimental line iutensities with CRE model predictions 
for an laser-produced aluminum plasma. In order to minimize uncertainties due to plasma opacity effects, I use the He-like $\left[1 s^{2}\left({ }^{1} S_{0}\right)-1 s 3 p\left({ }^{1} P_{1}\right)\right]$ "Hep" resonance line to the He-like $\left[1 s^{2}\left({ }^{1} S_{0}\right)-1 s 2 p\left({ }^{3} P_{1}\right)\right]$ "IC" intercombination line ratio to study the density dependence of the intercombination line. The CRE "He $\beta$ /IC" line ratio computed using the "RATION" code was shown earlier in Figure 21b for an optically thin and thick ( $50 \mu \mathrm{m}$ plasma size) plasma. Although the two spectral lines are not as directed coupled as the "He $\alpha$ " and "IC" lines, the CRE model indicates a definite density dependence in the " $\mathrm{He} / \mathrm{\Lambda C}$ " line invensity ratio.

\subsubsection{The Non-Stationary Density Dependence of the Resonance to Intercombination Line Ratio: A Quad-Steady State K-chell Model}

Boiko et al. (1983) have discussed the effects of a non-stationary plasma on the " $\alpha$ " line intensity ratio. In the typical stationary case, the ratio of the excitation rates from the ground state to the singlet and triplet levels approaches a limiting value of $R=1$ at low temperatures. The stationary value for $R$ increases with $T_{c}$ because the collisional mixing rate between the triplet levels decreases with the electron energy faster than the direct excitation rate for the singlet state. [Boiko et al. (1983)] For the recombining case, the plasma appears to be overionized relative to a given electron temperature and we would expect to find a much larger fraction of $H$-like ions than for a stationary CRE plasma. In this case, the $1 \mathrm{~s} 2 l$ configuration are fed by by recombinations of $\mathrm{H}$-like ions instead of by direct excitation from the $1 \mathrm{~s}^{2}\left({ }^{1} \mathrm{~S}_{0}\right)$ He-like ground state. Boiko et al. (1979) reported that the rate coefficients become proportional to the statistical weights of the levels and that $R=1 / 3$. This is based on 
considering only the direct recombination rates feeding the ${ }^{3} P_{1}$ and ${ }^{1} P_{1}$ excited levels. Actually, recombination effects are much more complex due to recombinations to neighboring excited states and the resulting cascades and collisional mixing between the level populations. The net result will he that recombinations preferentially feed the ${ }^{3} \mathrm{P}_{1}$ level relative to the ${ }^{1} \mathbf{P}_{1}$ level. But considering the simplified $n=2$ level case, Boiko finds that the " $\alpha$ " line intensity ratio can be determined from the expression:

$$
\alpha=\frac{1}{3}+\frac{N_{e} \cdot\left[\frac{16}{3} \cdot C\left(2^{3} \rightarrow 2^{1}\right)+\frac{4}{3} \cdot C\left(2^{3} \rightarrow i\right)\right]}{A\left(1 s^{3} p^{3} P_{1} \rightarrow 1 s^{21} S_{0}\right)}
$$

Boiko et al. (1979) also reports that for an underionized "overheated" plasma, the ${ }^{3} \mathrm{P}_{1}$ and $\mathrm{P}_{1}$ levels are populated predominantly by electrin collisionai ionization of the Li-like ions. In this case, the rates are proportional to the statiatical weights of the levels and once again $R=1 / 3$. In general, for a non-stationary plasma, there will likely be contributions to these levels by ionizations, recombinations, and excitations. The value of $R$ will typically be between $1 / 3$ and $\geq 1$ depending on the plasma (electron) temperature, and the relative He/FI and Li/He charge state fractions.

In general, the " $\alpha$ " line ratio should be just cne of many line intensity ratios which are analyzed simultaneously to characterize non-stationary plasmas. This procedure for analyzing data from a transient, ionizing or recombining plasma has been suggested by Boiko et al. (1984). In this method, several resonance and dielectror satellite lines are analyzed simultaneously and systematically to derived the relative ionization distribution and values for the electron density and temparature. A detailed, quasi-steady state, collisional radiative K-shell model is in development by 
W. Goldstein and A. Osterheld of LLNL and a preliminary version is used in this work. This model is briefly described here and used in Chapter 5 to analyze the aluminum K-shell data measured in this work. In the quasi-steady state model, the recombination and ionization processes are decoupled so that the relative charge state fraction can be solved for independently. The fastest dipole allowed radiative decay raies essentially set the time scale for establishing a quasi-steady state level population distribution. For $\mathrm{K}$-shell systems, this time scale is of order $0\left(10^{-13}\right.$ sec). Conversely, the recombination time scales are of order $\vartheta\left(10^{-10} \mathrm{sec}\right)$ to $\vartheta\left(10^{-9}\right.$ $\mathrm{sec}$ ) for the range of temperatures and densities typical to these laser plasmas and the charge state distributions appronch a quasi-steady state distribution on the $\sim 100 \mathrm{ps}$ time scale. [See discussion in Chapter 3 for important time scalelenf $\ldots$ s.] Therefore, we see that this quasi-steady state approach is valid only if the data used samples a time interval of order $\vartheta\left(10^{-11} \mathrm{sec}\right)$ to $\left.v^{\left(10^{-10}\right.} \mathrm{sec}\right)$, long enough to establish a steady state level population distribution and short enough to "freeze-in" a quasi-steady state charge state distribution. This final condition was met using the simultaneously space and time resolved ( $250 \mathrm{ps}$ time frames) FCXS data presented in Chapters 5 and 6.

The model represents the 215 lowest levels (1s ${ }^{2}$ ground state, the lowest 48 singly excited levels of the form $1 \mathrm{sn} /$, and the 166 doubly excited levels up to the $(2 \mathrm{p} 5 \mathrm{~g})$ configuration) in the He-like system as well as the 1 s H-like ground state. A more complete aluminum model including the remaining (n\5) $\mathrm{H}$-like states and $\mathrm{Li}-$-like contributions is in development by W. Goldstein and A. Osterheld (1988). The model is shown schernatically in Figure 26, showing a "blo d" energy level diagram and the important radiative and collisional transitions included in the model. In this detailed, quasi-steady state K-shell model, all of the atomic structure 
Figure 26. A schematic energy level diagram describing the important madiative and collisional processes that are included in the quasi-steady state $\mathrm{K}$-shell model. These include radiative, 3-body, and dielectronic recombination from the H-like ground state to the excited He-like staves. The collisional excitation from the $1 s^{2}\left({ }^{1} S_{0}\right)$ He-like ground state w the excited He-like states can also be independently accounted for. Full collisional mixis and radiative cascades between excited levels are included. The model contains the 1 s $\mathrm{H}$-like and $1 \mathrm{~s}^{2}\left({ }^{1} \mathrm{~S}_{0}\right) \mathrm{He}$-like ground states, the $48(1 \mathrm{en} l)$ sindly excited, and the $166(2 / \mathrm{N} /)$ doubly excited He-like states and assumes an cptically thin plasma. 


\section{He-like Recombination Model}

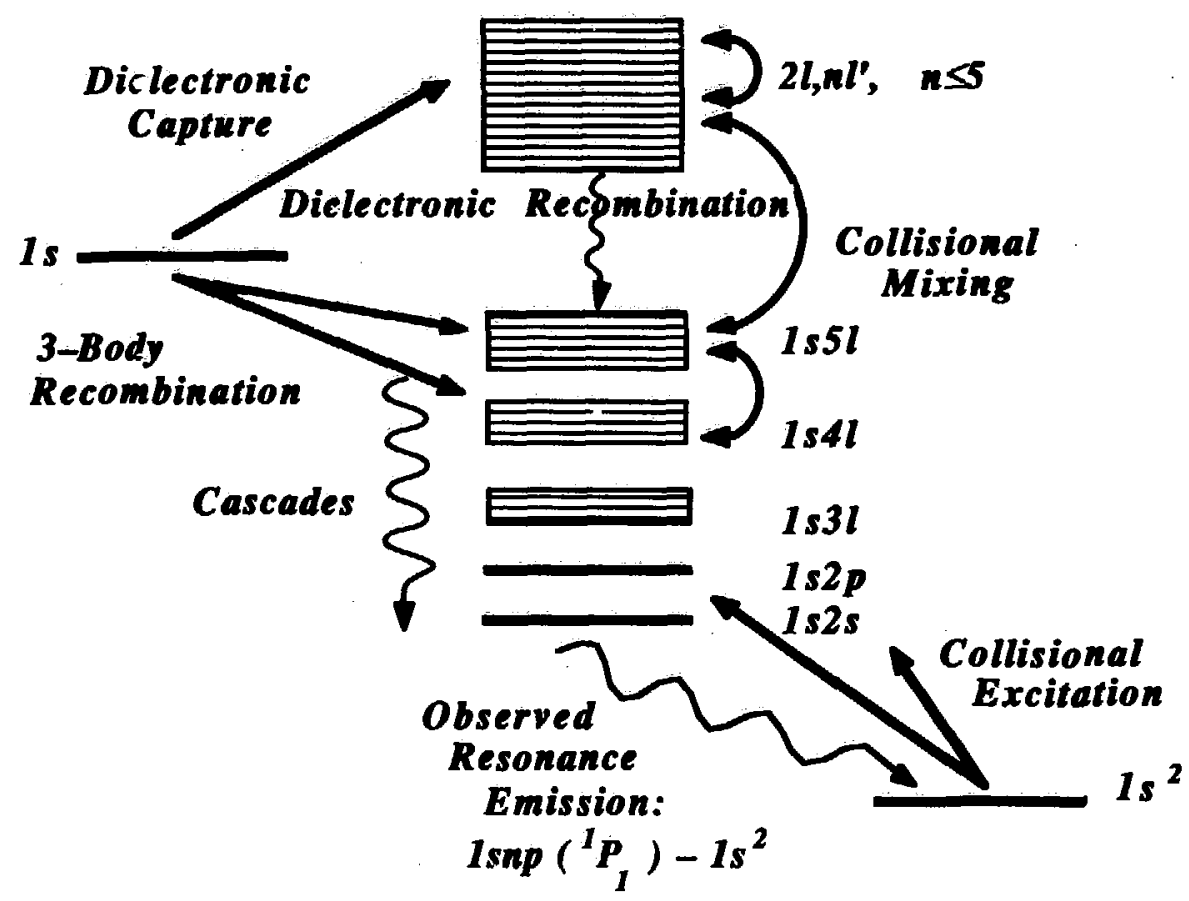


calculations (energy levels) and radiative decay rates (E1, E2, M1, and M2) are calculated using the multiconfigurational Relativistic Parametric Potential Model (RELAC) of Klapisch $(1971 ; 1977)$. All collisional transitions, including $\Delta n=0$ rates between excited states, are computed from the multiconfigurational, relativistic distorted wave (MCDW) calculation of Bar-Shalom et al. (1987). All other atomic processes coupling these levels are included explicitly and are based on an optically thin plasma and a collisional radiative scheme (radiative decays, cascades, and collisional excitation and deexcitations). Dielectronic recombination rates (autoionization and resonant capture rates for doubly-excited levels) were provided by M. Chen (1987) using his multiconfigurational Dirac-Fock "AUTOVAPP" code. Three body recombination rates were obtained via detailed balance with the collisional ionization Lotz formula. [Weisheit (1986); Cowan (1981)] Radiative recombination rates were derived from a detailed balance with photoionization rates calculated using the scaled, tydrogenic "PICS" code of J. Scofield (1988).

Unlike a stationary atomic model [such as the "RATION" (CRE) model], the relative charge state abundance is not set by a steady state ionization distribution determined by the electron density and tempenature. In this quasi-steady state $\mathrm{K}$-shell model, the relative abundances of $\mathrm{H}$-like and He-like ions (basically their ground atule populations) can be treated as independent "free" parameters and systematically varied until a satisfactory set of plasme conditions (electron temperature and density) and relative charge state fractions is obtained. In this "quasi-steady state" approach, the level population of the ith He-like level can be constructed from a set of linearly independent solutions representing a purely recombining plasma and a purely He-like plasma in which excited level populations originate primarily from collisional excitation from the $1 \mathrm{~s}^{2} \mathrm{He}$-like ground state. 
This linear solution is of the form:

$$
\begin{aligned}
& \frac{N(i)}{N\left(1 s^{2}\right)}=N^{\text {exc. }}(i)+N^{r e c} .(i) \cdot \frac{N(1 s)}{N\left(1 s^{2}\right)}, \\
& \text { where, } N^{\text {exc. }}(i)=\sum_{j=1 s^{2}}\left[\left(n_{e} Q\left(T_{e}\right)+A\right)^{-1}\right]_{i j} \cdot Q_{1 s^{2} \rightarrow j}\left(T_{e}\right), \\
& \text { and } N^{\text {rec. }}(i)=\sum_{j=1 s}\left[\left(n_{e} Q\left(T_{e}\right)+A\right)^{-1}\right]_{i j} \cdot R_{1 s \rightarrow j}\left(T_{e}\right)
\end{aligned}
$$

In the quasi-steady state rate equation above, $n_{e}$ and $T_{e}$ are the electron density and temperature, respectively, $\mathbf{Q}\left(\mathrm{T}_{\mathrm{e}}\right)$ is the matrix of collisional excitation and deexcitation rate coefficients with $Q_{i i}=\Sigma_{j} Q_{j i}$, and $A\left(T_{e}\right)$ is the matrix of radiative transition rates with $A_{i i}=-\Sigma_{j} A_{i j} . Q_{1 s^{2} \rightarrow j}$ is the vector of rate coefficients for collisional excitation of level $j$ from the $18^{2} \mathrm{He}$-like ground state and $\mathbf{R}_{1 \ell \rightarrow j}$ is the sum of recombination rate coefficients (radiative, 3-body, and dielectronic) from the 1s H-like ground state.

The " $\alpha$ " line ratio, for a predominantly recombining plasma (including radiative, 3-body, and dielectronic recombination), is plotted versus electron density in Figure 27(top) for a range of temperatures between 50 and $600 \mathrm{eV}$. For the recombinttion calculation, the He-like $1 \mathrm{~s}^{2}\left({ }^{1} \mathrm{~S}_{0}\right)$ ground state population was set equal to zero to minimize collisional excitation contributions to the upper excited state level populations. Instead, recombination contributions (recombinations to the H-like is ground state) is the primary population path populating the upper He-like levels. Subsequent radiative cascades and collisional mixing redistributes the excited electrons thus affecting the overall level population kinetics. The " $\alpha$ " line ratio for a 
Figure 27. The density dependence of the " $\alpha$ " line ratio is determined (top) for a purely recombining Al plasma and (bottom) for a purely He-like plasma populate by direct excitation from the $1 s^{2}\left({ }^{1} S_{0}\right)$ He-like ground state only. These " $\alpha$ " line intensity ratio predictions are from the quasi-steady state $\mathrm{K}$-shell model and represent a range of electron temperatures between 50 and $600 \mathrm{eV}$. 

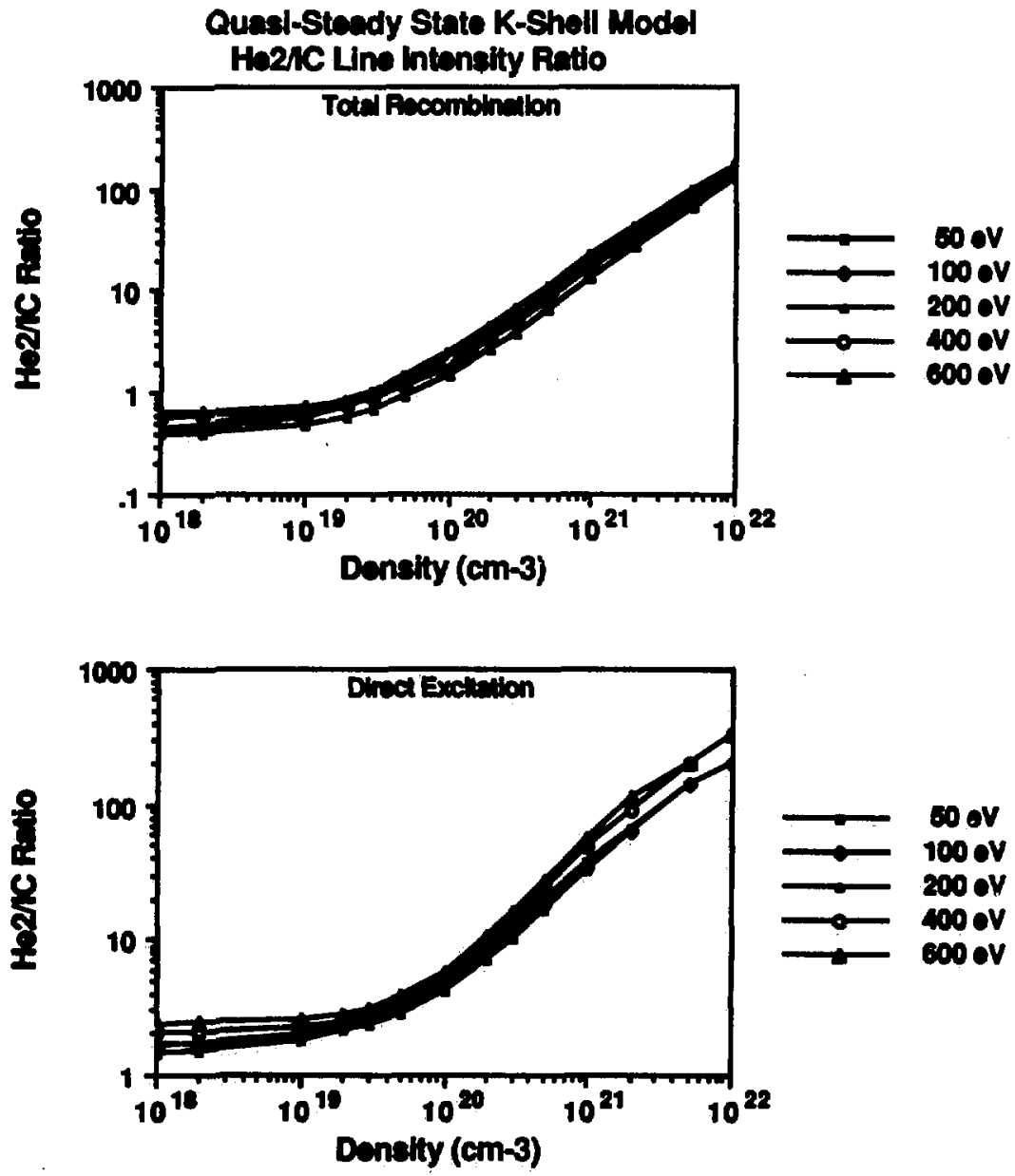

Figure 27 
purely direct excitation population scheme is also plotted in Figure 27(bottom). In this plot, the ${ }^{3} \mathrm{P}_{1}$ and ${ }^{1} \mathrm{P}_{1}$ excited levels are populated only by direct excitation from the ground state. For this case, the H-like ground state population was set equal to zero thus eliminating recombination processes to the He-like level populations.

The relative contributions of recombination processes and direct excitation are

shown in Figure 28. The quasi-steady state " $\alpha$ " line ratios for the purely recombining case, the purely direct excitation cuse, and from the "RATION" (CRE) model are plotted for an electron temperature of $200 \mathrm{eV}$. It should be noted that the CRE plot is very similar to the purely direct excitation value since at $200 \mathrm{eV}$, the CRE ionization distribution does not predict a significant H-like charge state fraction. Therefore, since there are very few hydrogen-like ions, the contribution to the CRE value of " $\alpha$ " from recombination processes is very small. The key factor is that for all electron densities, the pure recombination calculation predicts lower " $\alpha$ " line ratio values than either the pure excitation or CRE models. For densities below $n_{e} \leq 10^{20}$ $\mathrm{cm}^{-3}$, the CRE model " $\alpha$ " line intensity ratio is always greater than 1.3 while the recombination model predicts values of less than 1.0 for these densities and low temperatures. Thus, we see that the $1 \mathrm{~s} 2 \mathrm{p}\left(3 \mathrm{P}_{1}\right)$ excited level corresponding to the intercombination line is preferentially fed by recombinations. A telltale sign indicating a recombining plasma will be an intercombination line intensity approaching or exceeding the " $\mathrm{He}_{\alpha}$ " line intensity. In Chapter 5, the predicted density dependence of the " $\alpha$ " line ratio is tested using the new simultaneously space and resolved aluminum data. 
Figure 28. The predicted density depender ' 2 of the " $\alpha$ " line intensity ratio for a collisional radiative equilibrium Al plasma (via "RATION"), a purely recombining plasma, and a placma populated purely by direct excitation from the He-like ground, state are shown for an Al plasma at an electron temperature of $200 \mathrm{eV}$. 


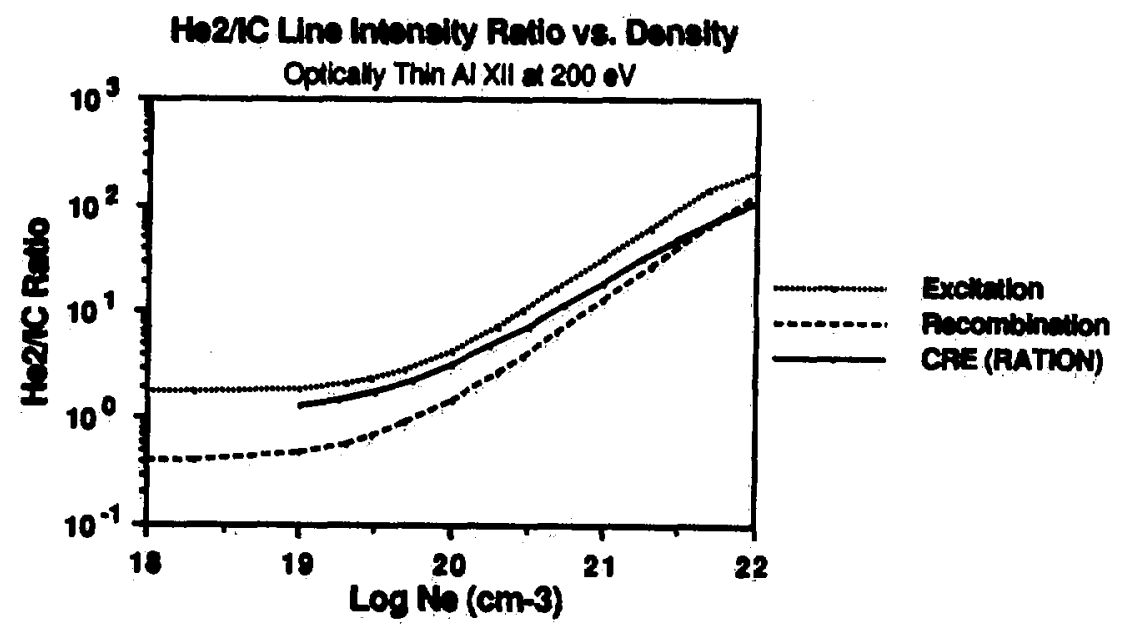

FIgure 28 


\subsection{Density Sensitive High-n He-like Resonance Lines.}

The He-like high-n resonance lines including the $\left[1 \mathrm{~s}^{2}\left({ }^{1} \mathrm{~S}_{0}\right)-1 \mathrm{~s} 3 \mathrm{p}\left({ }^{1} \mathrm{P}_{1}\right)\right]$ "He$\beta$ ",

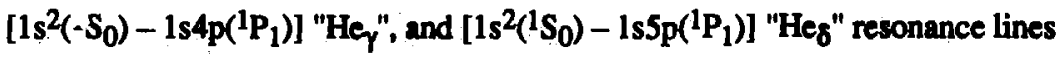
are also predicted to be sensitive to density variations for an overstripped, recombining plasmas. In these cases, the He-like excited level populations are originate primarily through recombination processes (mainly 3-body recombination) fran overstripped H-like ions which preferentially feed the high-n excited levels. Using the hydrogenic rates listeal in Table 1 (in Chapter 3), we see that the 3-body and radiative recombination rates scale approximately as:

$$
\frac{\beta^{3 B}(i)}{\beta^{r d}(i)} \propto n_{e} \cdot\left(T_{e}\right)^{-1 / 2} \cdot \frac{n^{7}}{Z^{6}} .
$$

Thus, for a cool, high density, recombining plasma, 3-body recombination should feed the upper (high-n) metastable levels significantly. We should also note that for low temperatures, the resonant capture rate cross section should be small so that dielectronic recombination is not a major popilation mechanism. This increased excited state population is then redistributed in the system due to radiative cascades and collisional mixing. For an overstripped, Al plasma at a temperature of $100 \mathrm{eV}$ and a density of $10^{21} \mathrm{~cm}^{-3}$, the He-like excited levels are initially populated by recombination from the H-like ground state. The He-like "high-lying" excited state population filters down to the "IC", "He ${ }_{\alpha}$ ", "Hes", "He$\gamma$ ", and"Hes" excited levels, due to subsequent collisional mixing and cascades. The flux (\%) contributions due to initial recombinations feeding the excited states, which then feed the "IC", "He ${ }_{\alpha}$ ", "Hep", "He $\gamma$ ", and"Hes" excited levels are listed in Table 3. At this low temperature 
Table 3. The flux (\%) contributions to the He-like a) "IC" - $\left.1 \mathrm{~s} 2 \mathrm{p}\left({ }^{3} \mathrm{P}_{1}\right) ; \mathrm{b}\right)$ " $\mathrm{He} \mathrm{e}_{\alpha}$ -

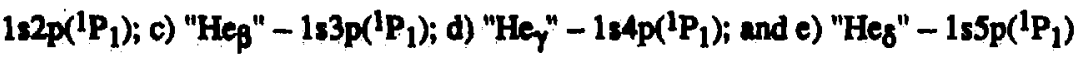
excited state level populations due to recombination processes calculated using the quasi-steady state $\mathrm{Al}$ model for a temperature of $100 \mathrm{eV}$ and and a density of $10^{21}$ $\mathrm{cm}^{-3}$. These values represent the flux contributions $(\geq 0.1 \%)$ into the specified excited state level which are due to collisional mixing and radiative cascades from other He-like levels. The primary population mechanism for these He-like levels is via recombinution (rediative, 3-body, and dielectronic) procesies from the 1s H-like ground state. It hould be noted that the flux contributions to the high-n He-like levels from lower lying levels are smell $(\leq 0.1 \%)$ due to the low electron temperature and the correspondingly low collisional excitation nate between the levels. 
Table 3a

IC Level: [1s2p(1/2,1/2)J=1]

Population (\%) due to Recombination:

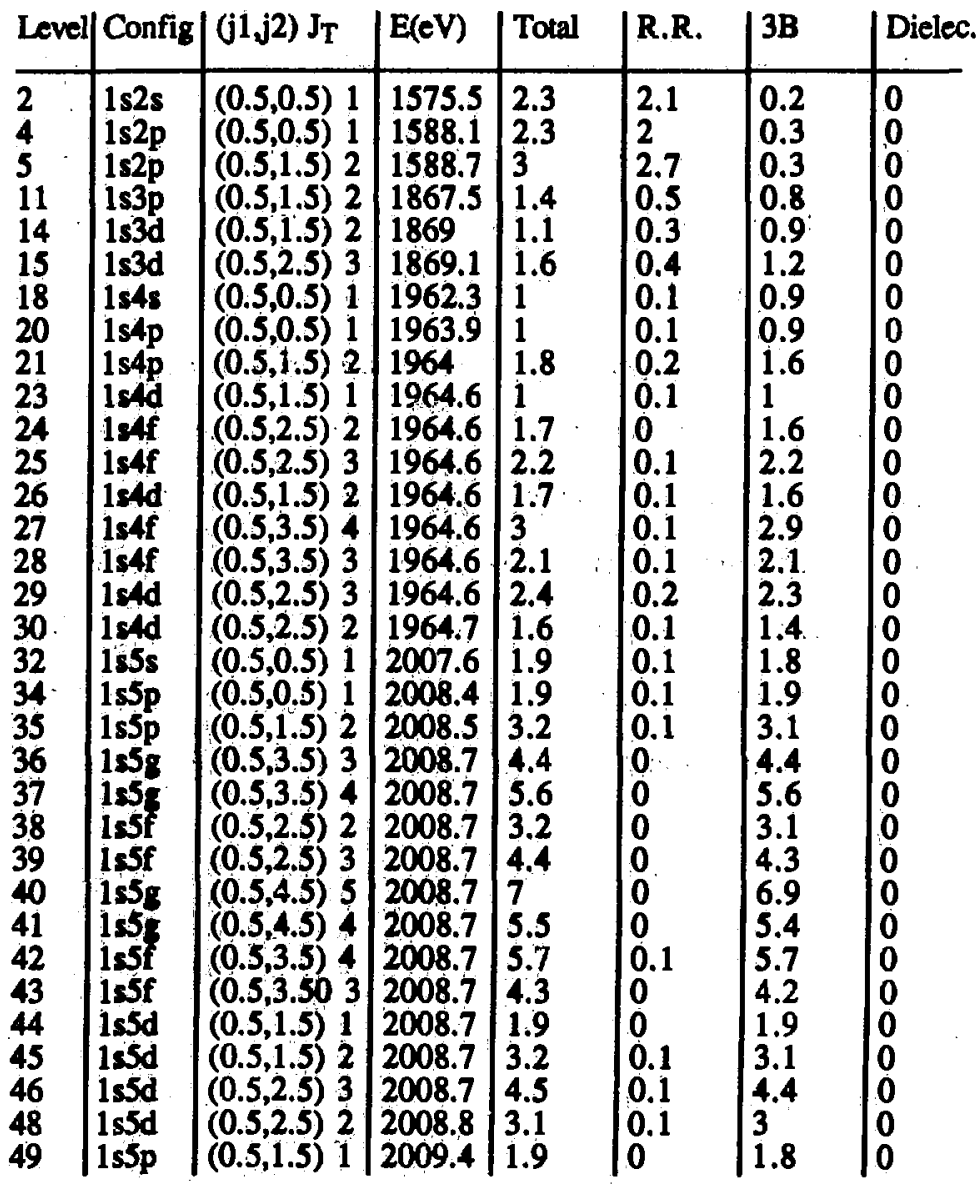


Table 3b

Hea Level: [1s2p(1/2,3/2)J=1]

Population $(\%)$ due to Recombination:

\begin{tabular}{|c|c|c|c|c|c|c|c|}
\hline Level! & Config & $(\mathbf{j} 1, j 2) J_{T}$ & $E(e V)$ & Total & R.R. & 3B & Dielec. \\
\hline $\begin{array}{l}2 \\
4 \\
5 \\
7 \\
11 \\
14 \\
15 \\
18 \\
20 \\
21 \\
23 \\
24 \\
25 \\
26 \\
27 \\
28 \\
29 \\
30 \\
32 \\
34 \\
35 \\
36 \\
37 \\
38 \\
39 \\
40 \\
41 \\
42 \\
43 \\
44 \\
45 \\
46 \\
48 \\
49\end{array}$ & 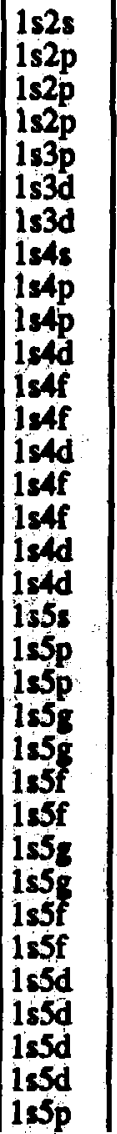 & 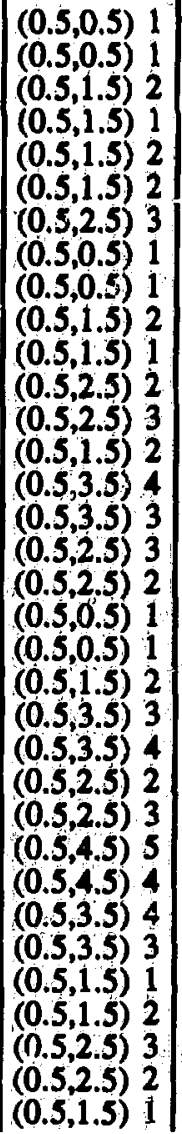 & $\begin{array}{l}1575.5 \\
1588.1 \\
1588.7 \\
1599.4 \\
1867.5 \\
1869 \\
1869.1 \\
1962.3 \\
1963.9 \\
1964 \\
1964.6 \\
1964.6 \\
1964.6 \\
1964.6 \\
1964.6 \\
1964.6 \\
1964.6 \\
1964.7 \\
2007.6 \\
2008.4 \\
2008.5 \\
2008.7 \\
2008.7 \\
2008.7 \\
2008.7 \\
2008.7 \\
2008.7 \\
2008.7 \\
2008.7 \\
2008.7 \\
2008.7 \\
2008.7 \\
2008.8 \\
2009.4\end{array}$ & $\begin{array}{l}1.7 \\
1.4 \\
2.4 \\
1.8 \\
1.2 \\
1 \\
1.5 \\
1 \\
1 \\
1.7 \\
1 \\
1.6 \\
2.2 \\
1.7 \\
2.9 \\
2.2 \\
2.4 \\
1.6 \\
1.9 \\
1.9 \\
3.2 \\
4.4 \\
5.6 \\
3.1 \\
4.4 \\
6.9 \\
5.5 \\
5.7 \\
4.3 \\
1.9 \\
3.2 \\
4.4 \\
3.1 \\
1.9\end{array}$ & $\begin{array}{l}1.6 \\
1.3 \\
2.1 \\
1.6 \\
0.5 \\
0.2 \\
0.4 \\
0.1 \\
0.1 \\
0.2 \\
0.1 \\
0 \\
0.1 \\
0.1 \\
0.1 \\
0.1 \\
0.2 \\
0.1 \\
0.1 \\
0.1 \\
0.1 \\
0 \\
0 \\
0 \\
0 \\
0 \\
0 \\
0.1 \\
0 \\
0 \\
0.1 \\
0.1 \\
0.1 \\
0.1\end{array}$ & $\begin{array}{l}0.2 \\
0.2 \\
0.3 \\
0.2 \\
0.8 \\
0.8 \\
1.1 \\
0.9 \\
0.9 \\
1.6 \\
0.9 \\
1.6 \\
2.2 \\
1.6 \\
2.9 \\
2.1 \\
2.2 \\
1.5 \\
1.8 \\
1.8 \\
3.1 \\
4.3 \\
5.6 \\
3.1 \\
4.3 \\
6.9 \\
5.5 \\
5.6 \\
4.3 \\
1.9 \\
3.1 \\
4.4 \\
3.1 \\
1.8\end{array}$ & $\begin{array}{l}0 \\
0 \\
0 \\
0 \\
0 \\
0 \\
0 \\
0 \\
0 \\
0 \\
0 \\
0 \\
0 \\
0 \\
0 \\
0 \\
0 \\
0 \\
0 \\
0 \\
0 \\
0 \\
0 \\
0 \\
0 \\
0 \\
0 \\
0 \\
0 \\
0 \\
0 \\
0 \\
0 \\
0\end{array}$ \\
\hline
\end{tabular}


Table 3c

He $\beta$ Level: [1s3p(1/2,3/2)J=1]

Population (\%) due to Recombination:

\begin{tabular}{|c|c|c|c|c|c|c|c|}
\hline evel & Config & $\mid(j 1, j 2) J_{T}$ & $\mathrm{E}(\mathrm{eV})$ & | Total & |R.R. & 3B & Dielec. \\
\hline $\begin{array}{l}2 \\
4 \\
5 \\
7 \\
11 \\
14 \\
15 \\
18 \\
20 \\
21 \\
23 \\
24 \\
25 \\
26 \\
27 \\
28 \\
29 \\
30 \\
32 \\
34 \\
35 \\
36 \\
37 \\
38 \\
39 \\
40 \\
41 \\
42 \\
43 \\
44 \\
45 \\
46 \\
48 \\
49\end{array}$ & 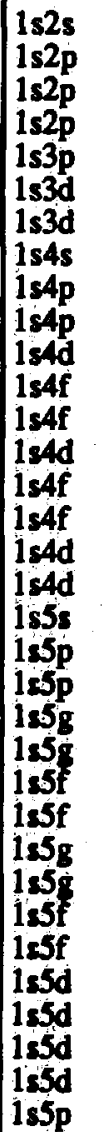 & 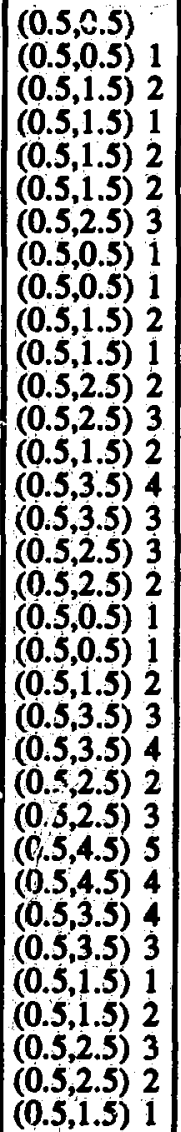 & $\begin{array}{l}1575.5 \\
1588.1 \\
1588.7 \\
1599.4 \\
1867.5 \\
1869 \\
1869.1 \\
1962.3 \\
1963.9 \\
1964 \\
1964.6 \\
1964.6 \\
1964.6 \\
1964.6 \\
1964.6 \\
1964.6 \\
1964.6 \\
1964.7 \\
2007.6 \\
2008.4 \\
2008.5 \\
2008.7 \\
2008.7 \\
2008.7 \\
2008.7 \\
2008.7 \\
2008.7 \\
2008.7 \\
2008.7 \\
2008.7 \\
2008.7 \\
2008.7 \\
2008.8 \\
2009.4\end{array}$ & $\begin{array}{l}1.7 \\
1.4 \\
2.4 \\
1.8 \\
1.2 \\
1 \\
1.5 \\
1 \\
1 \\
1.7 \\
1 \\
1.6 \\
2.2 \\
1.7 \\
2.9 \\
2.2 \\
2.4 \\
1.6 \\
1.9 \\
1.9 \\
3.2 \\
4.4 \\
5.6 \\
3.1 \\
4.4 \\
6.9 \\
5.5 \\
5.7 \\
4.3 \\
1.9 \\
3.2 \\
4.4 \\
3.1 \\
1.9\end{array}$ & $\begin{array}{l}1.6 \\
1.3 \\
2.1 \\
1.6 \\
0.5 \\
0.2 \\
0.4 \\
0.1 \\
0.1 \\
0.2 \\
0.1 \\
0 \\
0.1 \\
0.1 \\
0.1 \\
0.1 \\
0.2 \\
0.1 \\
0.1 \\
0.1 \\
0.1 \\
0 \\
0 \\
0 \\
0 \\
0 \\
0 \\
0.1 \\
0 \\
0 \\
0.1 \\
0.1 \\
0.1 \\
0.1\end{array}$ & $\begin{array}{l}0.2 \\
0.2 \\
0.3 \\
0.2 \\
0.8 \\
0.8 \\
1.1 \\
0.9 \\
0.9 \\
1.6 \\
0.9 \\
1.6 \\
2.2 \\
1.6 \\
2.9 \\
2.1 \\
2.2 \\
1.5 \\
1.8 \\
1.8 \\
3.1 \\
4.3 \\
5.6 \\
3.1 \\
4.3 \\
6.9 \\
5.5 \\
5.6 \\
4.3 \\
1.9 \\
3.1 \\
4.4 \\
3.1 \\
1.8\end{array}$ & $\begin{array}{l}0 \\
0 \\
0 \\
0 \\
0 \\
0 \\
0 \\
0 \\
0 \\
0 \\
0 \\
0 \\
0 \\
0 \\
0 \\
0 \\
0 \\
0 \\
0 \\
0 \\
0 \\
0 \\
0 \\
0 \\
0 \\
0 \\
0 \\
0 \\
0 \\
0 \\
0 \\
0 \\
0 \\
0\end{array}$ \\
\hline
\end{tabular}


Table 3d

He $\gamma$ Level: [1s4p(1/2,3/2)J=1]

Population (\%) due to Recombination:

\begin{tabular}{|c|c|c|c|c|c|c|}
\hline Level|Config & $\mid(j 1, j 2) J_{T}$ & $\mathrm{E}(\mathrm{eV})$ & | Total & | R.R. & |3B & Diele \\
\hline $\begin{array}{l}1 s 4 s \\
1 s 4 p \\
1 s 4 p \\
1 s 4 d \\
1 s 4 f \\
1 s 4 f \\
1 s 4 d \\
1 s 4 f \\
1 s 4 f \\
1 s 4 d \\
1 s 4 d \\
1 s 4 p \\
1 s 5 s \\
1 s 5 p \\
1 s 5 p \\
1 s 5 g \\
1 s 5 g \\
1 s 5 f \\
1 s 5 f \\
1 s 5 g \\
1 s 5 g \\
1 s 5 f \\
1 s 5 f \\
1 s 5 d \\
1 s 5 d \\
1 s 5 d \\
1 s 5 d \\
1 \mathrm{ssp}\end{array}$ & 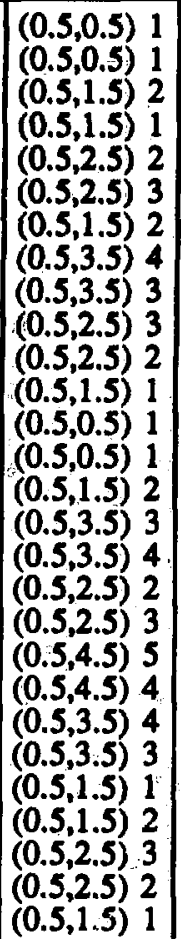 & $\begin{array}{l}1962.3 \\
1963.9 \\
1964 \\
1964.6 \\
1964.6 \\
1964.6 \\
1964.6 \\
1964.6 \\
1964.6 \\
1964.6 \\
1964.7 \\
1965.5 \\
2007.6 \\
2008.4 \\
2008.5 \\
2008.7 \\
2008.7 \\
2008.7 \\
2008.7 \\
2008.7 \\
2008.7 \\
2008.7 \\
2008.7 \\
2008.7 \\
2008.7 \\
2008.7 \\
2008.8 \\
2009.4\end{array}$ & $\begin{array}{l}1.1 \\
1.1 \\
1.8 \\
1 \\
1.7 \\
2.6 \\
1.9 \\
2.9 \\
2.9 \\
2.4 \\
2.5 \\
2 \\
2 . \\
2.1 \\
3.5 \\
4.8 \\
6.3 \\
3.4 \\
4.9 \\
7.4 \\
6.6 \\
6.1 \\
5.1 \\
2.1 \\
3.5 \\
4.8 \\
3.7 \\
2.2\end{array}$ & $\begin{array}{l}0.1 \\
0.1 \\
0.2 \\
0.1 \\
0 \\
0.1 \\
0.1 \\
0.1 \\
0.1 \\
0.2 \\
0.2 \\
0.2 \\
0.1 \\
0.1 \\
0.1 \\
0 \\
0 \\
0 \\
0.1 \\
0 \\
0 \\
0.1 \\
0.1 \\
0 \\
0.1 \\
0.1 \\
0.1 \\
0.1\end{array}$ & \begin{tabular}{|l}
1 \\
1 \\
1.6 \\
1 \\
1.6 \\
2.5 \\
1.8 \\
2.9 \\
2.8 \\
2.3 \\
2.3 \\
1.8 \\
2 \\
2 \\
3.4 \\
4.8 \\
6.3 \\
3.4 \\
4.9 \\
7.3 \\
6.5 \\
6 \\
5 \\
2 \\
3.5 \\
4.7 \\
3.6
\end{tabular} & $\begin{array}{l}0 \\
0 \\
0 \\
0 \\
0 \\
0 \\
0 \\
0 \\
0 \\
0 \\
0 \\
0 \\
0 \\
0 \\
0 \\
0 \\
0 \\
0 \\
0 \\
0 \\
0 \\
0 \\
0 \\
0 \\
0 \\
0 \\
0 \\
0\end{array}$ \\
\hline
\end{tabular}


Table $3 e$

He $\delta$ Level: [1s5p(1/2,3/2)J=1]

Population (\%) due to Recombination:

\begin{tabular}{|c|c|c|c|c|c|c|c|}
\hline Level & Config & $(\mathbf{j} 1, \mathbf{j} 2) \mathrm{J}_{\mathrm{T}}$ & $E(e V)$ & Total & R.R. & 3B & Dielec. \\
\hline $\begin{array}{l}21 \\
24 \\
25 \\
26 \\
27 \\
28 \\
29 \\
30 \\
31 \\
32 \\
34 \\
35 \\
36 \\
37 \\
38 \\
39 \\
40 \\
41 \\
42 \\
43 \\
44 \\
45 \\
46 \\
47 \\
48 \\
49\end{array}$ & $\begin{array}{l}1 s 4 p \\
1 s 4 f \\
1 s 4 f \\
1 s 4 d \\
1 s 4 f \\
1 s 4 f \\
1 s 4 d \\
1 s 4 d \\
1 s 4 p \\
1 s 5 s \\
1 s 5 p \\
1 s 5 p \\
1 s 5 g \\
1 s 5 g \\
1 s 5 f \\
1 s 5 f \\
1 s 5 g \\
1 s s g \\
1 s s f \\
1 s 5 f \\
1 s 5 d \\
1 s 5 d \\
1 s 5 d \\
1 s 5 s \\
1 s 5 d \\
1 s 5 p\end{array}$ & 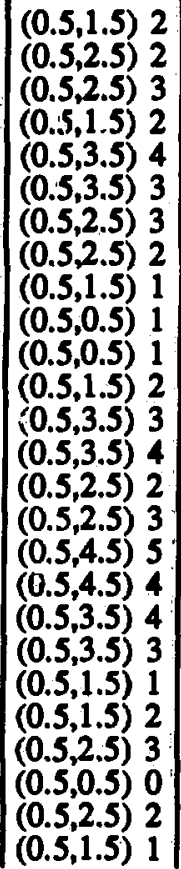 & $\begin{array}{l}1964 \\
1964.6 \\
1964.6 \\
1964.6 \\
1964.6 \\
1964.6 \\
1964.6 \\
1964.7 \\
1965.5 \\
2007.6 \\
2008.4 \\
2008.5 \\
2008.7 \\
2008.7 \\
2008.7 \\
2008.7 \\
2008.7 \\
2008.7 \\
2008.7 \\
2008.7 \\
2008.7 \\
2008.7 \\
2008.7 \\
2008.7 \\
2008.8 \\
2009.4\end{array}$ & $\begin{array}{l}1.6 \\
1.5 \\
2.1 \\
1.6 \\
2.7 \\
2.2 \\
2.2 \\
1.6 \\
1 \\
2.1 \\
2.2 \\
3.6 \\
4.9 \\
6.6 \\
3.5 \\
5.3 \\
7.5 \\
7.1 \\
6.2 \\
5.7 \\
2.2 \\
3.8 \\
5 \\
1.1 \\
4.5 \\
3.4\end{array}$ & $\begin{array}{l}0.2 \\
0 \\
0.1 \\
0.1 \\
0.1 \\
0.1 \\
0.2 \\
0.1 \\
0.1 \\
0.1 \\
0.1 \\
0.1 \\
0 \\
0 \\
0 \\
0.1 \\
0 \\
0 \\
0.1 \\
0.1 \\
0 \\
0.1 \\
0.1 \\
0 \\
0.1 \\
0.1\end{array}$ & $\begin{array}{l}1.5 \\
1.5 \\
2.1 \\
1.5 \\
2.6 \\
2.1 \\
2.1 \\
1.5 \\
0.9 \\
2.1 \\
2.2 \\
3.5 \\
4.9 \\
6.6 \\
3.5 \\
5.2 \\
7.4 \\
7.1 \\
6.2 \\
5.7 \\
2.1 \\
3.7 \\
4.9 \\
1.1 \\
4.4 \\
3.3\end{array}$ & $\begin{array}{l}0 \\
0 \\
0 \\
0 \\
0 \\
0 \\
0 \\
0 \\
0 \\
0 \\
0 \\
0 \\
0 \\
0 \\
0 \\
0 \\
0 \\
0 \\
0 \\
0 \\
0 \\
0 \\
0 \\
0 \\
0 \\
0\end{array}$ \\
\hline
\end{tabular}


$(100 \mathrm{eV}), 3$-body recombination is the major recombination process. We can also note that since 3-body recombination feeds the all the high-n levels, it is very important that the theoretical model be highly detailed in the excited state level structure and that collisional processes and radiative cascades be fully accounted for in the model. The radiative rates out of the excited levels corresponding to the

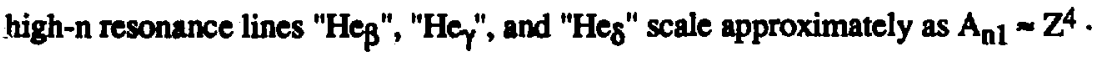
$n^{-5}$. Thus, radiative decay rates are larger for the lower $1 \operatorname{snp}\left({ }^{1} P_{1}\right)$ levels than for the higher lying metustable levels. The difference between the radiative decay rates and the 3-body recombination feeding rates suggests a population inversion between the high- $\mathrm{n}$ metastable levels may exist. This population inversion would be expected to manifest itself by the appearance of inverted $1 s^{2}\left({ }^{1} S_{0}\right)-1 \operatorname{snp}\left({ }^{1} P_{1}\right)$ resonance line intensities.

In fact, this is just the behavior predicted by the detailed, quasi-steady state K-shell model shown in Figure 29, for a pure recombination case and in Figure 30, for a pure He-like direct excitation case, respectively. Here, the "He//He $\beta$ " [top] and the "Heg/He $e_{B}$ " [bottom] line intensity ratios have been plotted versus the electron density for a range of temperatures between 50 and $600 \mathrm{eV}$ and show a "humped" behavior in the line intensity ratio indicating population inversions between the $1 s 4 p\left({ }^{1} P_{1}\right)$ and $1 \mathrm{~s} 3 \mathrm{p}\left({ }^{1} \mathrm{P}_{1}\right)$, and the $1 \mathrm{s5p}\left({ }^{1} \mathrm{P}_{1}\right)$ and $1 \mathrm{~s} 3 \mathrm{p}\left({ }^{1} \mathrm{P}_{1}\right)$ levels, respectively. Clearly, the recombination cases shown in Figure 30 indicate a significantly larger population inversion between the high-n metastable levels for the recombining plasma compare to the pure excitation case. This "humped" behavior in the high-n resonance line intensity ratios as a function of the electron density can be predicted by very simple arguments. At low densities $\left(\mathrm{n}_{\mathrm{e}} \geq 10^{19} \mathrm{~cm}^{-3}\right)$, the resonance line intensities are 
Figure 29. A comparison of the (top) "He//He $\beta$ " and the (bottom) "He $/ \mathrm{He}_{\beta}$ " line ratios for an: Al plasma populated only via recombination from the $H$-like ground state and subeequent collisional and radiative cascades between He-like excited states as predicted by the quasi-steady state $\mathrm{Al}$ model for a range of electron temperatires between 50 and $600 \mathrm{eV}$. 

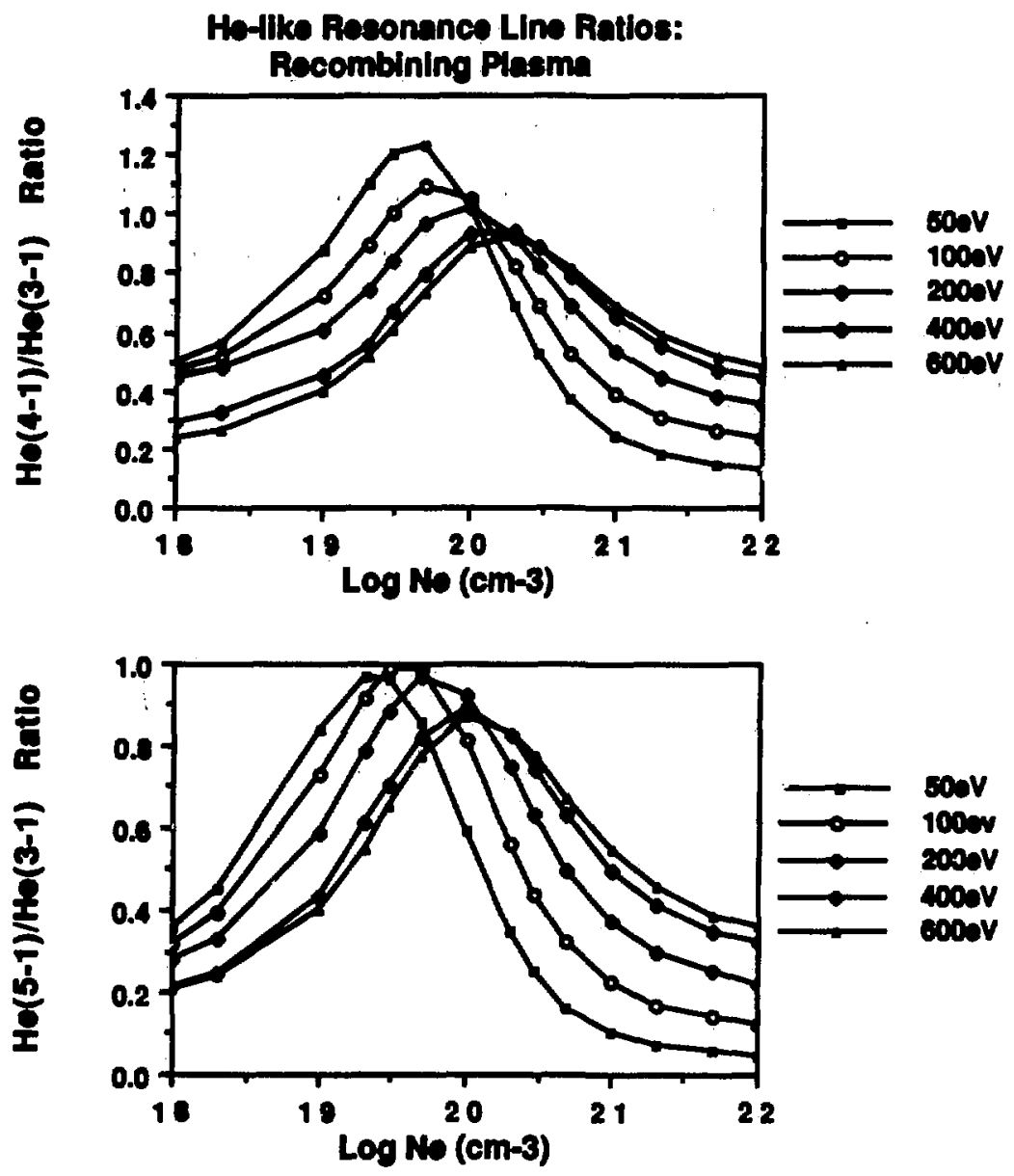

Figure 29 
Figure 30. A comparison of the (top) "He$/ \mathrm{He}_{\beta}$ " and the (bottom) "He $/ \mathrm{He}_{\beta}$ " line ratios for an Al plasma populated only via direct excitation from the He-like ground state and subsequent collisional and radiative cascades between He-like excited states as predicted by the quasi-steady state Al model for a range of electron temperatures between 50 and $600 \mathrm{eV}$. 

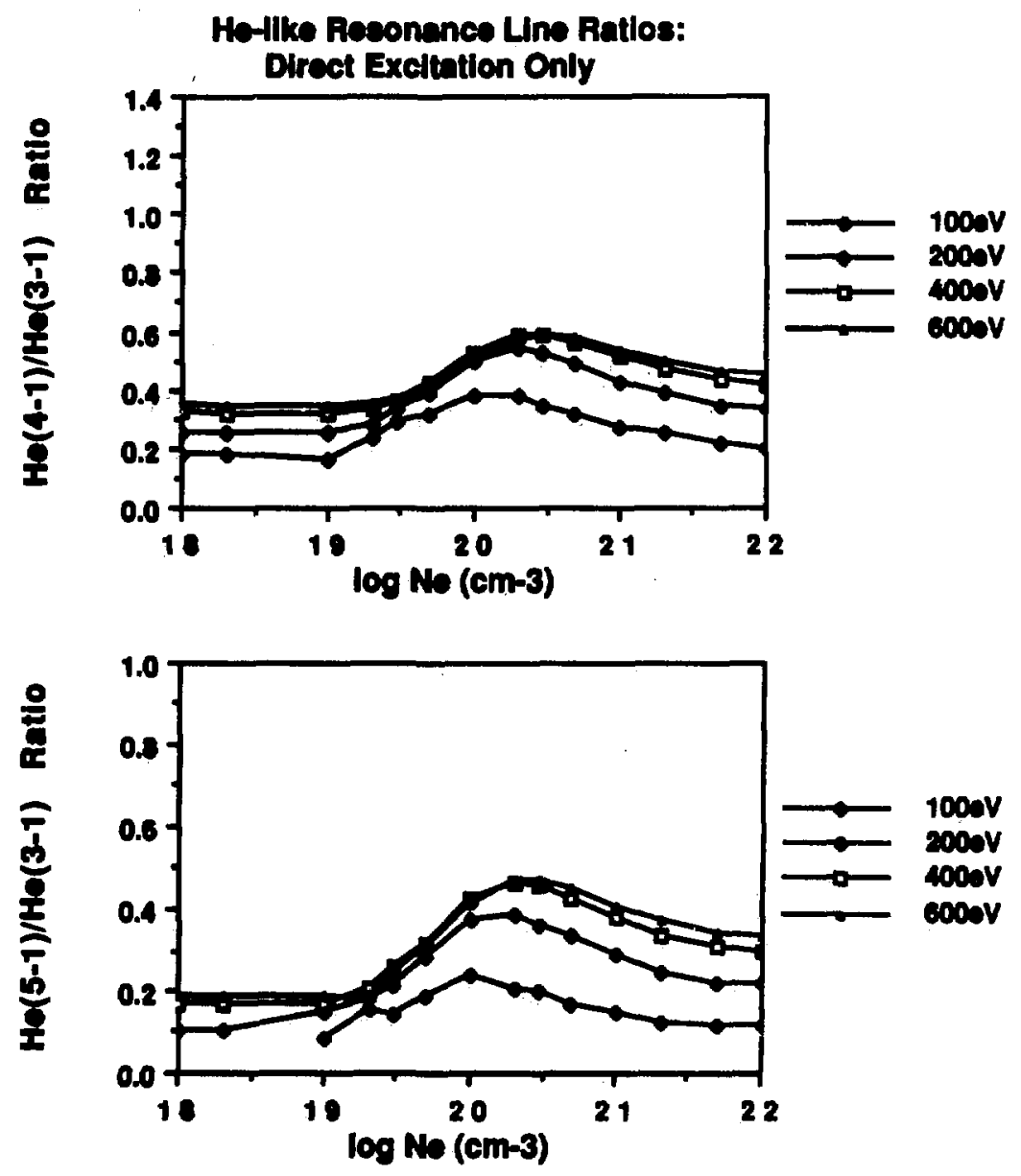

Figure 30 
governed strictly by the relative collisional excitation rates from the He-like ground state to the high-n excited levels. At very high densities $\left(n_{e} \geq 10^{22} \mathrm{~cm}^{-3}\right)$, the level populations approach Local Thermodynamic Equilibrium (LTE) and all the levels are populated statistically. For densities just below the LTE limit, the higher-n levels will nearer to LTE and populated statistically while the lower-n levels will be somewhat further from LTE. The higher-n level will have a larger population and therefore, its relative line intensity $\left[A_{i j} \cdot N(i)\right]$ will be stronger than the lower-n resonance line. For less dense plasmas, the 3-body recombination rate is strong (due to the $\mathrm{n}_{e}^{2}$ dependence) and the high-n levels will be preferentially populated as described earlier. This trend continues until the density is low enough such that 3-body recombination is significantly reduced (relative to excitation rates) and the population inversion is depleted. Furthermore, as shown in Figures $31 \mathrm{a}$ and 31b, the relative abundance of He-like to $\mathrm{H}$-like must be extremely large $\left[\mathrm{N}\left(1 \mathrm{~s}^{2}\right) / \mathrm{N}(1 \mathrm{~s})\right.$ $\gg 100]$ in order for the direct excitation population mechanisms to compete with the recombination processes generating the inverted high-n resonance line intensity ratios at $100 \mathrm{eV}$ and $200 \mathrm{eV}$, respectively.

Several key points should be made. This behavior is predicted in part due to the nature of the quasi-steady state analysis in which the relative charge state abundances are treated as free parameters. The standard CRE model would have predicted a specific charge state distribution as prescribed by electron temperature and density (assuming CRE). As discussed previously, at low temperatures ( $\sim 100 \mathrm{ev})$, the CRE charge state distribution is weighted towards He-like with very little $\mathrm{H}$-like. Therefore, the corresponding recombination contribution feeding the He-like excited levels is very small and the population inversion as indicated by the predicted (recombination model) resonance line ratios would likely not be seen. In other words, if there is no H-like, there will be no (or very little) He-like formed by 
Figure 31. A comparison of the (top) "He $/ \mathrm{He}_{\beta}$ " and (bottom) "He $/ \mathrm{He}_{\beta}$ " line ratios for a plasma populated by recombination or direct excitation as predicted by the quasi-steady state Al model. The calculation shows that the recombination processes feeding these levels are a) 100,000 times as strong as the excitation processes at $100 \mathrm{eV}$ and b) 100 times as strong as the excitation processes at $200 \mathrm{eV}$. 

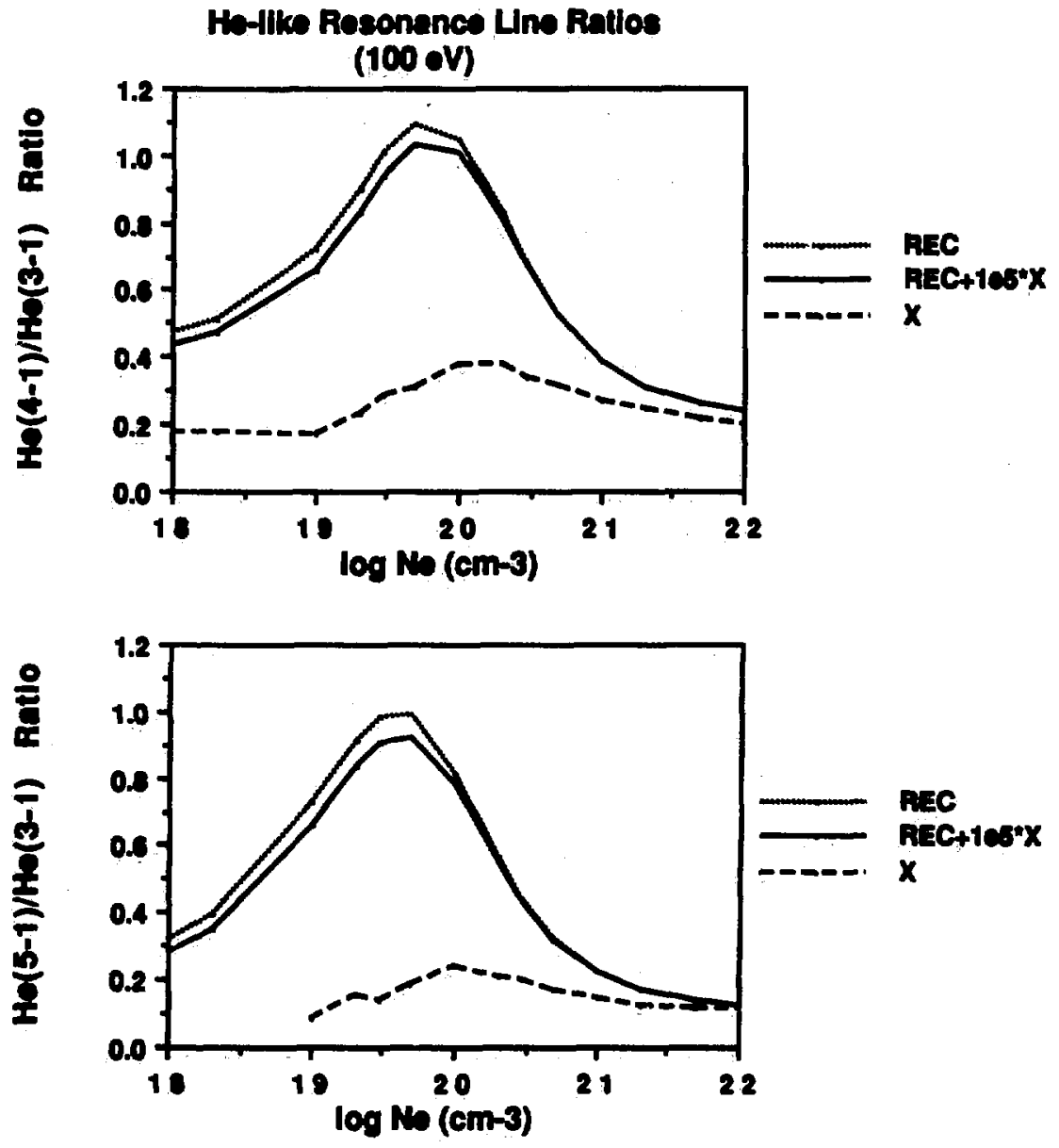

Figure 31a 

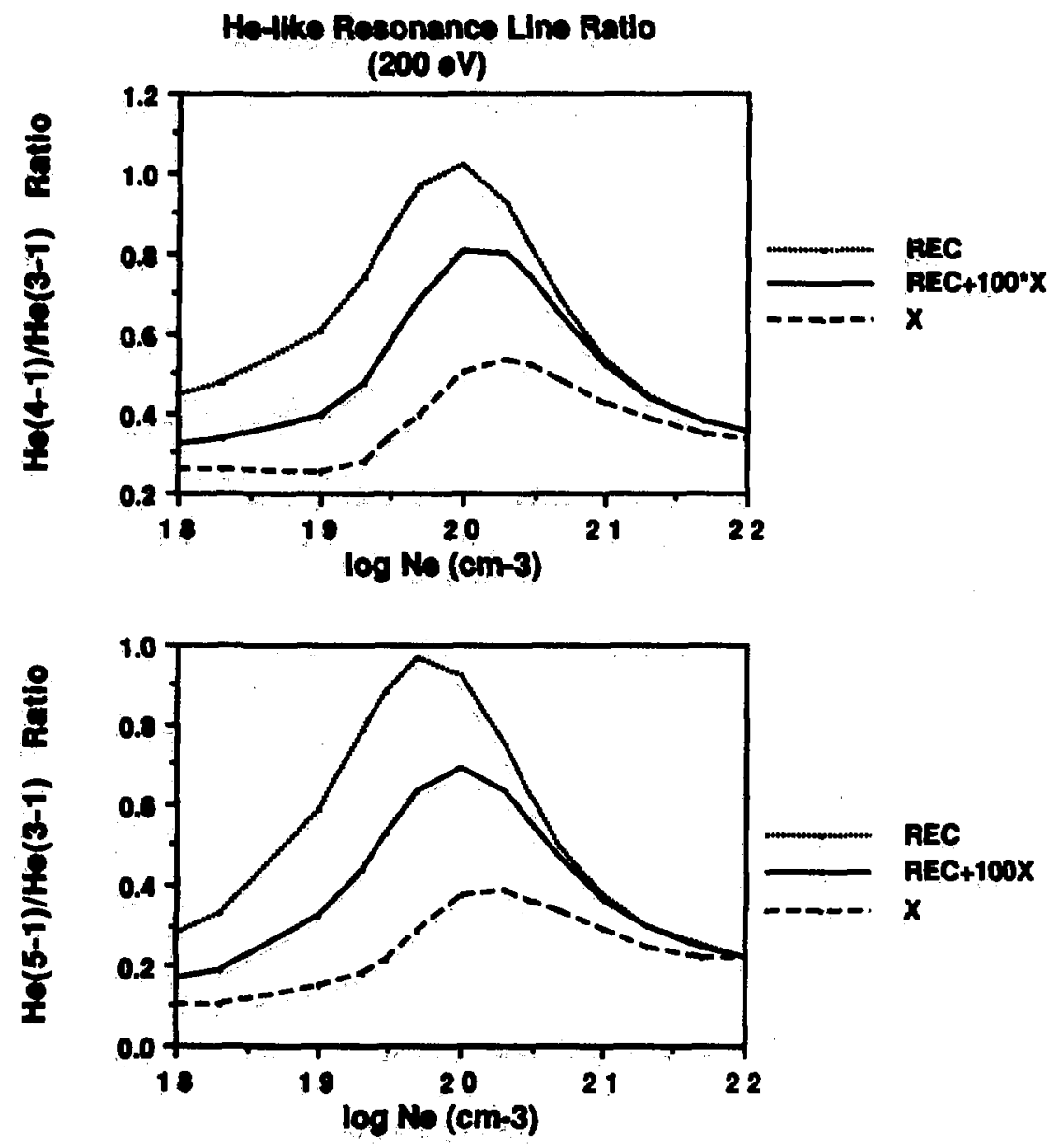

Figure 31b 
recombination processes.

Additionally, the quasi-steady state model used in this analysis is fully detailed in including all levels up to the ( $2 \mathrm{p5g}$ ) configuration and fully accounts for all collisional mixing and radiative cascades among the excited levels in the He-like system. The "RATION" (CRE) model is based on a collisional radiative equilibrium charge state distribution and uses single "lumped" terms of the form (1snp) for $n \geq 3$ levels to simplify the overall model. As shown in Figure 21g, the same high-n He-like resonance line intensity ratios obtained from the "RATION" model do not show the "humped" inverted line ratio behavior as predicted by the more detailed, quasi-steady state (pure recombination and pure excitation) model predictions. The reason for the "RATION" (CRE) model not showing this "humped" resonance line ratio trend and therefore the predicting the corresponding population inversion between the He-like metastable states is not known. This does indicate that detailed, quasi-stendy state atomic models which provide for all population mechanisms (direct excitation, recombination, and ionization) and which fully accounts for collisional mixing and ridiative cascades between excited levels are required to model non-stationary laser-produced plasmas.

\subsection{The Electric Quadrupole to Resonance Line Ratio: Denuity Variation of $(n=2)$ to $(n=3)$ Lines in Ne-like Ions}

High-Z, Laser-produced Ne-like jons have been the focus of recent studies towards the development of jhort wavelength lasing schemes. Matthews et al. (1985) first demonstrah amplified spontaneous emission in $\Delta n=0$ transitions in the $n=3$ singly excited states of Ne-like selenium at $266.3 \AA$ and $209.6 \AA$ using a high-powered, laser-heated "exploding foil" target design. Since then, amplified spontakeous emission based on similar laser-produced Ne-like plasmas experiments have been 
demonstrated in Ne-like copper and germanium [T. Lee et al. (1987); Elton et al. (1987)], selenium, yttrium, and molybdenum [Matthews et al. (1985; 1987); MacGowan et al. (1987)]. The lower lasing levels are the $\left.\left[2 s^{2} 2 p^{5} 3 s(3 / 2,1 / 2)\right]=1\right]$ or $\left[2 s^{2} 2 p^{5} 3 x(1 / 2,1 / 2) J=1\right]$ which decay rapidly to the ground state via dipole-allowed radiative transitions. I remind the reader that throughout this section, I refer to the Ne-like levels using the electron configuration (dropping the $1 \mathrm{~s}^{2}$ term) along with the $\left(j_{1}, j_{2}\right)$ and $J_{\text {towd }}$ for each level. The upper $\left[2 s^{2} 2 p^{5} 3 p(3 / 2,1 / 2) \mathrm{J}=2\right]$, $\left.\left[2 s^{2} 2 p^{5} 3 p(3 / 2,3 / 2)\right]=2\right],\left[2 s^{2} 2 p^{5} 3 p(1 / 2,1 / 2) J=0\right]$ and $\left[2 s^{2} 2 p^{5} 3 p(1 / 2,3 / 2) J=2\right]$ metastable levels decay vir much slower electric quadrupole transitions and may be affected by collisional processes which reduce the desired population inversion. In a similar fashion, these collisional processes will affect other metastable levels in the Ne-like system. Therefore, it is important that the theoretical atomic models ueed to model these short-wavelength lasers and Ne-like ions in general, correctly sccount for these collisional processes between the excited states. Despite the success of these short-wavelength laser experiments, there are many discrepancies between the experimental duta and theoretical predictions. The precise populating mechanism generating the observed amplified spontaneous emission in these laser-produced Ne-like systems remains a botly debated issue and draws attention to the great importance in understanding the complex atomic physics of highly ionized ions. [Apruzese et al (1985); Goldstein et al (1987b; 1987c); Griem (1986); Hagelstein et al (1986); Rosen et al. (1985; 1987); and Whitten et al (1986)] Thus independent experiments to characterize Ne-like laser-produced plasmas are needed in order to test these theoretical models and to ensure that the complex atomic processes are properly inclinded in the theory.

As shown in the previous sections, the populations of metastable levels in He-like ions are highly sensitive to variations in the electron density. Similar density 
sensitive metastable levels exjst in Ne-like ions. In this section, I describe the density dependence of metastable levels in excited Ne-like ions as predicted by the two steady state, collisional radiative Ne-like atomic models. These models are "NELI", a steady state, 37 level, collisional radiative Ne-like model developed by $R$. Walling (1988) and a second, more detailed, steady state, 89 level Ne-like collisional radiative model of W. Goldstein and A. Otterheld (1988). I particular, I examine the density dependence of the $\left.\left[2 s 2 p^{6} 3 d(1 / 2,5 / 2)\right]=2\right],\left[2 s 2 p^{6} 3 p(1 / 2,3 / 2) J=1\right]$, and $\left[2 s^{2} 2 p^{5} 3,(3 / 2,1 / 2) \mathrm{J}=1\right]$ excited levels in Ne-like $\mathrm{Mo}^{32+}$ and $\mathrm{Ag}^{37+}$ and the characteristic $x$-rays emitted by radiative decays of theace excited levels to the Ne-like ground state. The x-ray transitions from these excited levels to the Ne-like ground state can be observed experimentally. In Chapter 6, I test both Ne-like atomic models by comparing experimental to theoretical line intensity ratios ofer a range of electron densities determined independently using simultaneous laser interferometric measurements.

\subsection{The "NELI" 37 level Colliaional Radiative Model}

"NELI" is a compuler code written by R. Walling (1988) which calculates the Ne-like intensities based on a steady state, collisional radiative Ne-like electron-impuct excitation model. The model contuins the ground state $\left[2,22 p^{6}(0,0)\right.$ $J=0$ ] and the lowest $36(n=3)$ singly excived states. This is a "Z-scaled" model in which calculations can be made for Ne-like ions between argon and xenon (18 $\leq \mathrm{Z} \leq$ 54). In this collisional madiative model, the excited levels are populated by electron impact exciation from tive ground state as well as ndiative and collisionally decays from higher energy states. The excited levels are depopulated by ratiative and collisional decays to other excited levels as well as radiative transitions to the ground 
state for some levels. Rate equations for each the population of each level cen be written in the form:

$$
\frac{d N_{i}}{d t}=0=N_{0} \cdot C_{0 i}+\sum_{j>i}\left(A_{j i}+C_{j i}\right) \cdot N_{j}-\sum_{j<i}\left(A_{i j}+C_{i j}\right) \cdot N_{i}
$$

where $\mathrm{N}_{0}$ is the ground state population, and $\mathrm{N}_{\mathrm{i}}$ and $\mathrm{N}_{\mathrm{j}}$ are excited level populations and $A_{i j}$ and $C_{i j}$ are radiative and collisional transition rates between levels, respectively. The level populations are calculated by solving the system of rate equations based on a steady state balance between the radiative decay rates and the electron collision rates. The model considers only Ne-like ions and does not handle ionization or recombination processes. Also, no plasma opacity effects are considered in the model. A schematic representation of the lowest energy levels of a typical Ne-like system is presented in Figure 32. The relative levels in the schematic diagram do not correspond to any particular energy value and all of the excited energy levels are not shown.

$\mathrm{Ab}$ initio energy levels and spontaneous radiative rates (E1) were obtained from $\mathrm{J}$. Scofield (1987) using the relativistic (term averaged) configuration interaction "RAC" computer code which uses Dirac-Slater states. Higher order radiative decay rates (E2, Mi1, M2) were obtained from M. Chen (1987) using Grant's relativistic atomic structure code which is a multiconfigurational Dirac-Fcck model with intermediate coupling. Collision cross sections (ground state to excited state) are calculated via distorted-wave Born approximation for all 36 transitions. [A. Hazi (1987), i.. Reed (1987), P. Hagelstein (1987)] A classical Gaunt factor approximation (Bethe approximation) based on El radiative rates computed from Scofield's RAC code is used for all dipole-allowed collisional transitions between excited states [van Regemorter (1962)] : 
$-165-$

Figure 32. The energy level diagram representing the lowest $n=3$ singly excited levels of the Ne-like ion. 


\section{Schematic Representation of the lower energy levels of the No-like ion.}

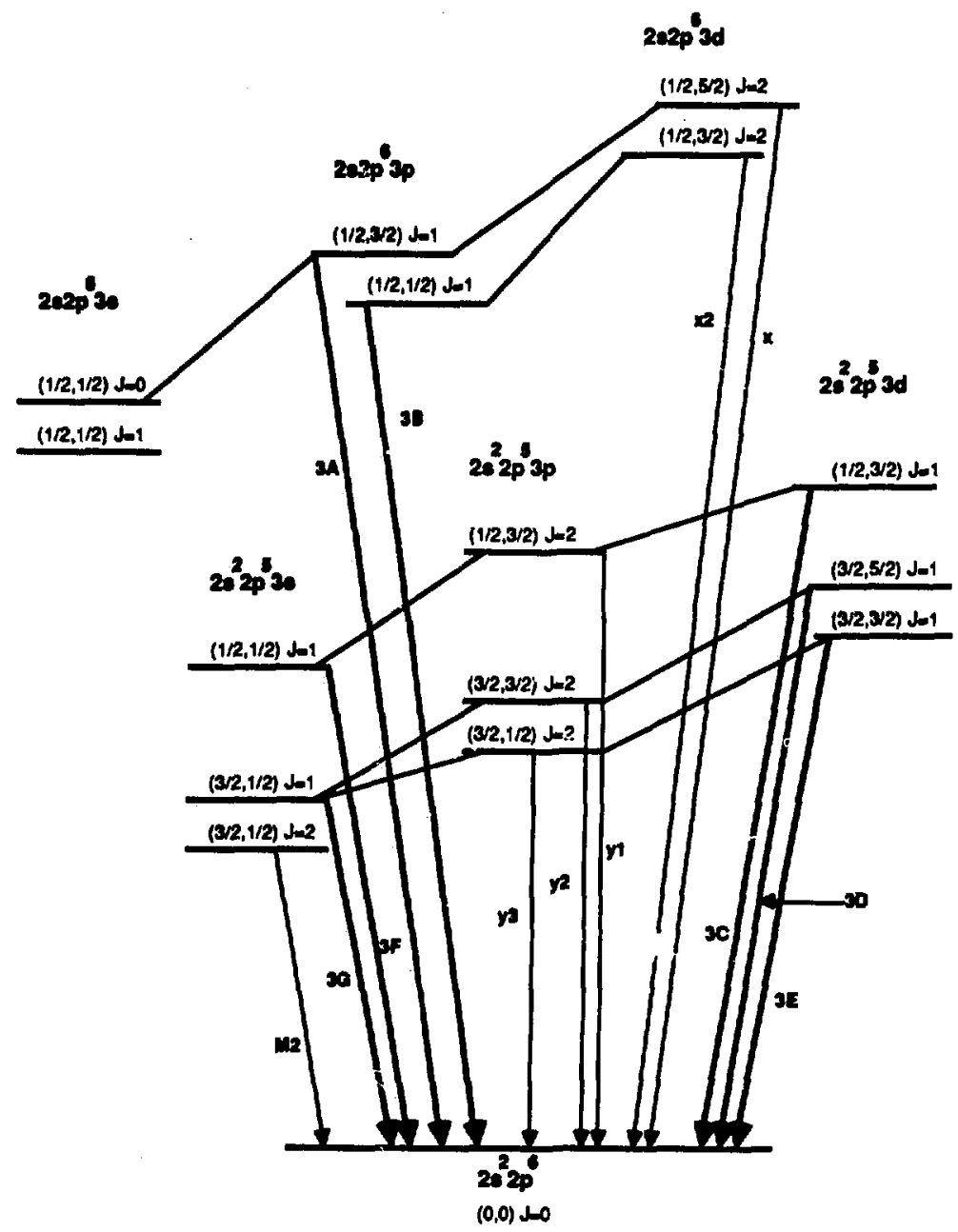

Figure 32 


$$
C_{i j}=\frac{8 \cdot \pi}{\sqrt{3}} \cdot\left(\pi \cdot a_{o}^{2}\right) \cdot \frac{1}{k_{i}^{2}} \cdot \frac{I_{H}}{\left(E_{j}-E_{i}\right)} \cdot f_{j i} \cdot \bar{g}
$$

where $K_{i}$ is the initial energy (in units of $13.6 \mathrm{eV}$ ) of the free electron, $\mathrm{I}_{\mathrm{H}}$ is the hydrogenic threshold ionization energy, $f_{\mathrm{ij}}$ is the oscillator strength, and $\langle g\rangle$ is the effective Gaunt factor.

In this collisional radiative model, seven of the $36(n=3)$ Ne-like excited states can decay via dipole-allowed (E1) $x$-ray emission. The remaining states decay radiatively by $\Delta \mathrm{n}=0$ dipole allowed transitions and for some levels, via "forbidden" transitions (E2, M1, \& M2) to the ground state. The wavelengths and radiative decay rates for the $\mathrm{E} 1$ and $\mathrm{E} 2 \mathrm{X}$-ray transitions for $\mathrm{Mo}^{32+}$ and $\mathrm{Ag}^{37+}$ are listed in Table 4. As the density increases, the model predicts that many metastable excited state populations (with long radiative lifetimes) are collisionally mixed to other levels. This collisional "mixing" depopulates the metastable levels and redistributes their population to other excited states. This is shown in Table 5 which lists the important radiative and collisional rates and fluxes $(\%)$ into and out of the excited levels: $\left[2 s 2 p^{6} 3 d(1 / 2,5 / 2) J=2\right],\left[2 s 2 p^{6} 3 p(1 / 2,3 / 2) J=1\right]$, and $\left[2 s^{2} 2 p^{5} 3 s(3 / 2,1 / 2) J=1\right]$ in Ne-like Mo32+. These excited levels correspond to the upper levels of the " $x$ " electric quadrupole line, and the " $3 \mathrm{~A}$ " and "3G" resonance lines, respectively, which will be discussed in greater detail later in this chapter. These values were computed based on $\mathrm{T}_{\mathrm{e}}=500 \mathrm{eV}, \mathrm{n}_{\mathrm{e}}=10^{21} \mathrm{~cm}^{-3}$, and assuming an optically thin plasma. 
Table 4. The Ne-like $\mathrm{Mo}^{32+}$ and $\mathrm{Ag}^{37+}(\mathrm{n}=3)$ to $(\mathrm{n}=2)$ electric dipole and quadrupole excited state level configurations, wavelengths, and radiative decay rates are listed. 
Ne-like E1 \& E2 X-ray Transitions

Molybdenum $(Z=42)$ Silver $(Z=47)$

Transition ${ }^{\dagger}$ Upper Level $\quad \lambda(\dot{A})^{*} \Delta\left(\sec ^{-1}\right) \quad \lambda(\dot{A})^{*} \Delta\left(\sec ^{-1}\right)$

E2 "x"t† $2 \mathrm{~s} 2 \mathrm{p}^{6} 3 \mathrm{~d}(1 / 2,5 / 2)_{2} \quad 4.2731 \quad 3.0 \times 10^{11} \quad 3.2987 \quad 6.2 \times 10^{11}$

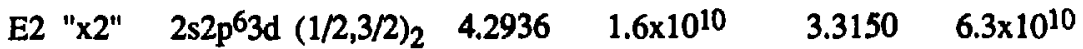

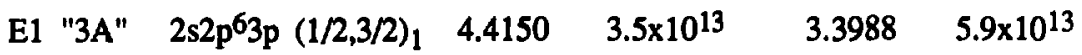

$\begin{array}{llllll}\text { E1 "3B" } & 2 \mathrm{~s} 2 \mathrm{p}^{6} \mathrm{p} \mathrm{p}(1 / 2,1 / 2)_{1} & 4.4618 & 1.2 \times 10^{13} & 3.4447 & 2.2 \times 10^{13}\end{array}$

E1 "3C" $2 \mathrm{~s}^{2} 2 \mathrm{p}^{5} 3 \mathrm{~d}(1 / 2,3 / 2)_{1} \quad 4.6305 \quad ? .8 \times 10^{14} \quad 3.5466 \quad 2.8 \times 10^{14}$

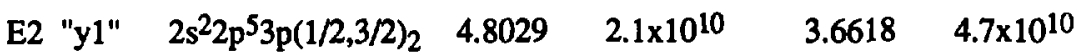

E1 "3D" $2 \mathrm{~s}^{2} 2 \mathrm{p}^{5} 3 \mathrm{~d}(3 / 2,5 / 2)_{1} \quad 4.8045 \quad 1.8 \times 10^{14} \quad 3 . .76 \quad 3.3 \times 10^{14}$

$\begin{array}{llllll}\text { E1 "3E" } & 2 \mathrm{~s}^{2} 2 \mathrm{p} 53 \mathrm{~d}(3 / 2,3 / 2)_{1} & 4.8609 & 1.2 \times 10^{11} & 3.7606 & 4.5 \times 10^{10}\end{array}$

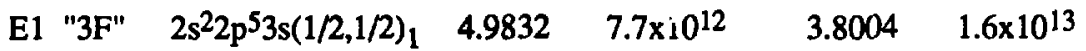

$\begin{array}{llllll}\text { E2 "y2" } & 2 \mathrm{~s}^{2} 2 \mathrm{p}^{5} 3 \mathrm{p}(3 / 2,3 / 2)_{2} & 5.0089 & 1.9 \times 10^{10} & 3.8629 & 5.0 \times 10^{10}\end{array}$

E2 "y3" $2 \mathrm{~s}^{2} 2 \mathrm{p}^{5} 3 \mathrm{p}(3 / 2,1 / 2)_{2} \quad 5.0731 \quad 1.9 \times 10^{10} \quad 3.9252 \quad 5.6 \times 10^{10}$

$\begin{array}{llllll}\text { E1 "3G" } 2 \mathrm{~s}^{2} 2 \mathrm{p} 53 \mathrm{~s}(3 / 2,1 / 2)_{1} & 5.2078 & 1.1 \times 10^{13} & 4.0191 & 1.8 \times 10^{13}\end{array}$

tUsing the notation of Burkhalter et al. (1975) for the Ne-like resonance lines.

†tUsing the notation of Gauthier et al. (1986).

"Wavelengths ar,j transition rates provided by Scofield (1987) and Chen (1987). 
Table 5. The collisional and radiative rates and fluxes feeding the a) "x" excited state level, [2s2p63d(1/2,5/2)J=2]; b) "3A" excited state level, [2s2p63p(1/2,3/2)J=1]; and c) "3G" excited state level, [2s $\left.\left.22 p^{5} 3 s(J / 2,1 / 2)\right]=1\right]$, have been calculated using the 37 level "NELI" model for $\mathrm{Mo}^{32+}$ at a temperature of $500 \mathrm{eV}$ and density of $10^{21} \mathrm{~cm}^{-3}$. The Ne-like levels feeding the "x", "3A", and "3G" states have been listed using a shortened notation. For level $1, " 2 s^{2} 2 p^{6 "}$ corresponds to the Ne-like ground state. The "2p3s", "2p3p", and " $2 \mathrm{p} 3 \mathrm{~d}$ " terms, correspond to the $2 s^{2} 2 p^{5} 3 s, 2 s^{2} 2 p^{5} 3 p$, and $2 s^{2} 2 p^{5} 3 d$ level configurations, respectively, Similarly, "2s3s", "2s3p", and "2s $3 d^{\prime}$ are the $2 \mathrm{~s} 2 \mathrm{p} 63 \mathrm{~s}, 2 \mathrm{~s} 2 \mathrm{p}^{6} 3 \mathrm{p}$, and $2 s 2 p^{6} 3 d$ level configurations, respectively. For all electron level configurations, the $1 \mathrm{~s}^{2}$ term not been listed. In the third column, the $\left(j_{1}, j_{2}\right)$ and $J_{\text {total }}$ for each level is listed. 
" $x^{n}$ Level: $2 s 2 p^{63 d}(1 / 2,5 / 2) J=2$

Radiative and Collisional Rates(sec-1)/Fluxes(\%) to the " $x$ " Level:

\begin{tabular}{|c|c|c|c|c|c|c|c|c|c|c|}
\hline Leve & $\mid$ Config & $\left(\mathbf{j}_{1}, \mathbf{j}_{2}\right) \mathbf{J}_{\mathbf{T}}$ & $\mathrm{A}_{\mathrm{ij}}(t)$ & + Flux & $A_{i j}(-)$ & t-Flux & $\mid C_{i j}(+)$ & + Flux & $C_{i j}(-)$ & -Flux \\
\hline $\begin{array}{l}1 \\
2 \\
3 \\
10 \\
13 \\
15 \\
16 \\
18 \\
19 \\
22 \\
24 \\
25 \\
26 \\
27 \\
31 \\
32 \\
33\end{array}$ & $\begin{array}{l}2 s^{2} 2 p^{6} \\
2 p 3 s \\
2 p 3 s \\
2 p 3 s \\
2 p 3 d \\
2 p 3 d \\
2 p 3 d \\
2 p 3 d \\
2 p 3 d \\
2 p 3 d \\
2 p 3 d \\
2 p 3 d \\
2 p 3 d \\
2 p 3 d \\
2 s 3 p \\
2 s 3 p \\
2 s 3 p\end{array}$ & $\begin{array}{ll}(0,0) 0 & 0 \\
(1.5,0.5) & 2 \\
(1.5,0.5) & 1 \\
(0.5,4.5) & 1 \\
(1.5,1.5) & 1 \\
(1.5,1.5) & 3 \\
(1.5,1.5) & 2 \\
(1.5,2.5) & 2 \\
(1.5,2.5) & 3 \\
(1.5,2.5) & 1 \\
(0.5,1.5) & 2 \\
(0.5,2.5) & 2 \\
(0.5,2.5) & 3 \\
(0.5,1.5) & 1 \\
(0.5,0.5) & 1 \\
(0.5,1.5) & 2 \\
(0.5,1.5) & 1\end{array}$ & & & $\begin{array}{l}3.10 c+11 \\
3.50 c+06 \\
8.40 c+08 \\
8.30 c+08 \\
2.60 c+09 \\
6.70 c+10 \\
1.00 c+09 \\
1.60 c+11 \\
1.40 c+11 \\
6.40 c+10 \\
8.40 c+09 \\
1.60 c+10 \\
5.40 e+10 \\
5.30 c+09 \\
8.40 c+09 \\
4.50 c+08 \\
2.90 c+10\end{array}$ & \begin{tabular}{|l}
16.4 \\
\\
0.1 \\
3.7 \\
0.1 \\
8.8 \\
7.7 \\
3.5 \\
0.5 \\
0.9 \\
3.0 \\
0.3 \\
0.5
\end{tabular} & $\begin{array}{l}6.70 c+07 \\
1.40 c+05 \\
5.70 e+07 \\
1.40 c+08 \\
8.20 c+08 \\
9.30 c+09 \\
2.00 e+08 \\
3.30 c+10 \\
2.20 c+10 \\
2.80 e+10 \\
6.30 c+09 \\
1.30 c+10 \\
3.20 e+10 \\
8.10 c+09 \\
9.60 c+10 \\
6.70 e+09 \\
8.00 c+11\end{array}$ & $\begin{array}{l}0.2 \\
3.9 \\
0.1 \\
7.1 \\
9.0 \\
0.1 \\
0.7 \\
2.2 \\
5.1 \\
0.8 \\
0.4 \\
2.9\end{array}$ & $\begin{array}{l}4.50 c+09 \\
4.00 e+05 \\
9.60 c+07 \\
1.90 e+08 \\
1.00 e+09 \\
2.60 e+10 \\
4.00 e+08 \\
6.60 c+10 \\
6.00 e+10 \\
3.10 e+10 \\
1.00 e+10 \\
2.10 c+10 \\
7.20 e+10 \\
7.60 c+09 \\
7.40 e+10 \\
8.20 e+09 \\
5.80 e+11\end{array}$ & $\begin{array}{l}0.2 \\
\\
0.1 \\
1.4 \\
3.6 \\
3.3 \\
1.7 \\
0.6 \\
1.1 \\
4.0 \\
0.4 \\
4.1 \\
0.5 \\
31.8\end{array}$ \\
\hline
\end{tabular}


Table 5b

"3A" Level: $2 \operatorname{sip} 3 p(1 / 2,3 / 2) J=1$

Radiative and Collisional Rates(sec-1)/Fluxes(\%) to the "3A" Level:

\begin{tabular}{|c|c|c|c|c|c|c|c|c|c|c|}
\hline & Config & $\left(\mathbf{j}_{1}, \mathbf{j}_{2}\right) \mathrm{J}_{\mathrm{T}}-$ & $A_{i j}(t)$ & + Flux & $A_{i j}(-)$ & |-Flux & $C_{i j}(+)$ & +Elux & $C_{\mathrm{jj}}(-)$ & |-Flux \\
\hline $\begin{array}{l}1 \\
4 \\
5 \\
7 \\
8 \\
11 \\
14 \\
20 \\
21 \\
23 \\
28 \\
29 \\
34 \\
35\end{array}$ & $\begin{array}{l}2 s^{2} 2 p^{6} \\
2 p 3 p \\
2 p 3 p \\
2 p 3 p \\
2 p 3 p \\
2 p 3 p \\
2 p 3 p \\
2 p 3 p \\
2 p 3 p \\
2 p 3 p \\
2 s 3 s \\
2 s 3 s \\
2 s 3 d \\
2 s 3 d \\
2 s 3 d\end{array}$ & \begin{tabular}{|ll}
$(0,0) 0$ & \\
$(1.5,0.5)$ & 1 \\
$(1.5,0.5)$ & 2 \\
$(1.5,1.5)$ & 1 \\
$(1.5,1.5)$ & 2 \\
$(1.5,1.5)$ & 0 \\
$(0.5,0.5)$ & 1 \\
$(0.5,0.5)$ & 0 \\
$(0.5,1.5)$ & 1 \\
$(0.5,1.5)$ & 2 \\
$(0.5,0.5)$ & 1 \\
$(0.5,0.5)$ & 0 \\
$(0.5,1.5)$ & 1 \\
$(0.5,1.5)$ & 2 \\
$(0.5,2.5)$ & 2
\end{tabular} & $\begin{array}{l}2.10 e+09 \\
1.10 e+09 \\
2.90 e+10\end{array}$ & & $\begin{array}{l}3.50 c+13 \\
1.40 c+09 \\
1.80 c+09 \\
1.90 c+11 \\
2.00 c+11 \\
7.80 e+10 \\
1.60 c+09 \\
1.10 c+10 \\
2.10 c+10 \\
6.50 c+10 \\
1.10 c+10 \\
1.80 c+10\end{array}$ & $\begin{array}{l}93.1 \\
0.5 \\
0.5 \\
0.2 \\
\\
0.1 \\
0.2\end{array}$ & $\begin{array}{l}1.50 c+07 \\
2.20 c+08 \\
1.80 c+08 \\
4.10 c+10 \\
2.70 c+10 \\
6.80 c+10 \\
8.90 c+08 \\
2.80 c+10 \\
1.80 c+10 \\
3.40 c+10 \\
1.60 c+11 \\
1.30 c+12 \\
7.20 c+10 \\
3.60 c+10 \\
5.80 c+11\end{array}$ & $\begin{array}{l}3.0 \\
6.1 \\
4.7 \\
\\
2.5 \\
1.4 \\
2.7 \\
4.3 \\
36.9 \\
1.4 \\
1.1 \\
23.5\end{array}$ & $\begin{array}{l}1.40 c+09 \\
4.60 e+08 \\
6.20 c+08 \\
8.10 c+10 \\
8.80 c+10 \\
4.20 c+10 \\
1.50 c+09 \\
1.50 c+10 \\
2.90 c+10 \\
9.00 c+10 \\
2.00 c+11 \\
5.20 c+11 \\
6.20 c+10 \\
5.20 c+09 \\
8.00 c+11\end{array}$ & $\begin{array}{l} \\
\\
0.2 \\
0.2 \\
0.1 \\
\\
0.1 \\
0.2 \\
0.5 \\
1.4 \\
0.2 \\
0.1 \\
2.1\end{array}$ \\
\hline
\end{tabular}


"3G" Level: $2 s^{2} 2 p^{53}(3 / 2,1 / 2) J=1$

Radiative and Collisional Raves(sec-1)/Fluxes(\%) to the "3G" Level:

\begin{tabular}{|c|c|c|c|c|c|c|c|c|c|c|}
\hline Leve! & Config & $\left(j_{1}, j_{2}\right) J_{r}$ & $A_{i j}(t)$ & + Elux & $A_{i j}(-)$ & -Flux & $C_{i j}(t)$ & +Flux & $\mid c_{i j}(-)$ & -Flux \\
\hline $\begin{array}{l}1 \\
4 \\
5 \\
7 \\
8 \\
9 \\
11 \\
14 \\
20 \\
21 \\
23 \\
28 \\
29 \\
34 \\
35 \\
37\end{array}$ & $\begin{array}{l}2 s^{2} 2 p^{6} \\
2 p^{3} p \\
2 p 3 p \\
2 p 3 p \\
2 p 3 p \\
2 p 3 s \\
2 p 3 p \\
2 p 3 p \\
2 p 3 p \\
2 p 3 p \\
2 p 3 p \\
2 s 3 s \\
2 s 3 s \\
2 s 3 d \\
2 s 3 d \\
2 s 3 d\end{array}$ & 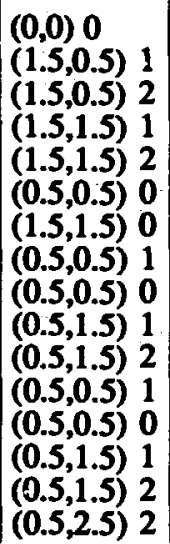 & $\begin{array}{l}5.40 c+08 \\
5.40 c+09 \\
2.80 c+11 \\
1.80 c+10 \\
1.20 c+07 \\
7.20 c+10 \\
9.10 c+07 \\
2.50 c+10 \\
2.00 c+08 \\
6.10 c+07 \\
9.50 c+10 \\
4.10 c+11 \\
1.40 c+06 \\
5.70 c+05 \\
8.40 c+08\end{array}$ & $\begin{array}{l}0.4 \\
0.6 \\
1.3 \\
1.5 \\
0.7\end{array}$ & $1.10 c+13$ & 85.6 & $\begin{array}{l}2.00 e+07 \\
-4.00 e+10 \\
3.50 c+11 \\
6.40 e+11 \\
3.50 c+11 \\
1.60 e+08 \\
7.20 c+11 \\
3.00 c+08 \\
5.40 c+10 \\
4.10 e+08 \\
1.20 c+08 \\
4.30 c 10 \\
1.60 e+11 \\
1.80 e+05 \\
7.20 c+04 \\
9.60 c+07\end{array}$ & $\begin{array}{l}4.6 \\
2.5 \\
26.6 \\
14.4 \\
24.4 \\
15.3 \\
1.5 \\
\\
0.3 \\
1.4\end{array}$ & $\begin{array}{l}7.80 e+08 \\
3.60 c+10 \\
5.10 c+11 \\
5.40 e+11 \\
4.80 c+11 \\
4.40 e+07 \\
1.90 c+11 \\
2.10 c+08 \\
1.20 c+10 \\
2.70 c+08 \\
1.40 c+08 \\
2.20 c+10 \\
2.70 e+10 \\
6.50 c+04 \\
4.30 c+04 \\
5.70 c+07\end{array}$ & $\begin{array}{l}0.3 \\
0.4 \\
4.3 \\
3.8 \\
1.5 \\
0.1 \\
\\
0.2 \\
0.2\end{array}$ \\
\hline
\end{tabular}




\subsubsection{Density Sensitive Meiastable Levels in Ne-like $\mathrm{Mo}^{32+}$ Ions.}

The density dependence of the various Ne-like $\mathrm{Mo}^{32+}$ lines is shown in Figure 33, for the region from $10^{19}$ to $10^{22} \mathrm{~cm}^{-3}$, as computed by the "NELI" model at an electron temperature of $500 \mathrm{eV}$. In this plot, the relative line intensities of various $n=3$ to $n=2$ transitions for Ne-like Mo (as a fraction of the total $n=3$ to $n=2$ rate) have been plotted versus the electron density. For relatively low density plasmas, the electric quadrupole (and other "forbidden") transitions have radiative decay rates large enough to compete with collisional deexcitation tates and may be observed along with the dipole-allowed, E1 transitions in the $n=3$ to $n=2 x$-ray emission spectra. The $\mathrm{E} 2$ transitions are more prominent in higher $\mathrm{Z}$ plasmas due to the $\mathrm{Z}$-scaling of these rates (approximately $\mathrm{Z}_{\mathrm{eff}} 6$ versus $\mathrm{Z}_{\mathrm{eff}}{ }^{4}$ for $\mathrm{E} 1$ transitions where $Z_{\text {eff }}$ is the effective charge of the Ne-like ion). [Weisheit (1986)] In addition, there is a "critical" density at which the upper level for each transitions begins to collisionally depopulate. This critical density is quite low for the M1 and M2 transitions which have been observed only in low density tokamak plasmas by Rice et al. (1988) and Beiersdorfer et al. $(1986,1987)$ and in the solar corona. [Smith (1985)] As the density increases, the collisional radiative model predicts that the upper levels for each E1 and E2 transition begin to collisionally depopulate to other excited states. The E2 transitions are affected at lower electron densities than E1 transitions due to their slower radiative decay times.

In Figure 33, we see that the ":r" e'stric quadrupole line intensity is roughly constant from $10^{19}$ to $10^{20} \mathrm{~cm}^{-3}$, then decreases monotonically between $10^{20}$ and $10^{23} \mathrm{~cm}^{-3}$. We can also examine the radiative and collisional fluxes into and out of the " $x$ ", the "3A", and the " $3 G$ " excited levels at a density of $10^{21} \mathrm{~cm}^{-3}$ predicted by the same "NELI" model at $500 \mathrm{eV}$. [See Table 5a.] The " $x$ " level is populated 
Figure 33. The density dependence of various Ne-like lines predicted by the 37 level "NELI" model. The standard $(n=3)$ to $(n=2)$ resonance lines are labeled " $a$ " to " $\mathrm{g}$ " while the electric dipole lines are labeled " $\mathrm{x"}$, " $\mathrm{x} 1$ " for the (3d-2s) transitions and " $y 1$ ", " $y 2$ ", and " $y 3$ " for the (3p-2p) transitions. The " $x$ ", "3A" (labeled " $a ")$, and "3G" (labeled " $\mathrm{g}$ ") line intensities have been shown in bold print and are discussed in Chapter 6. 


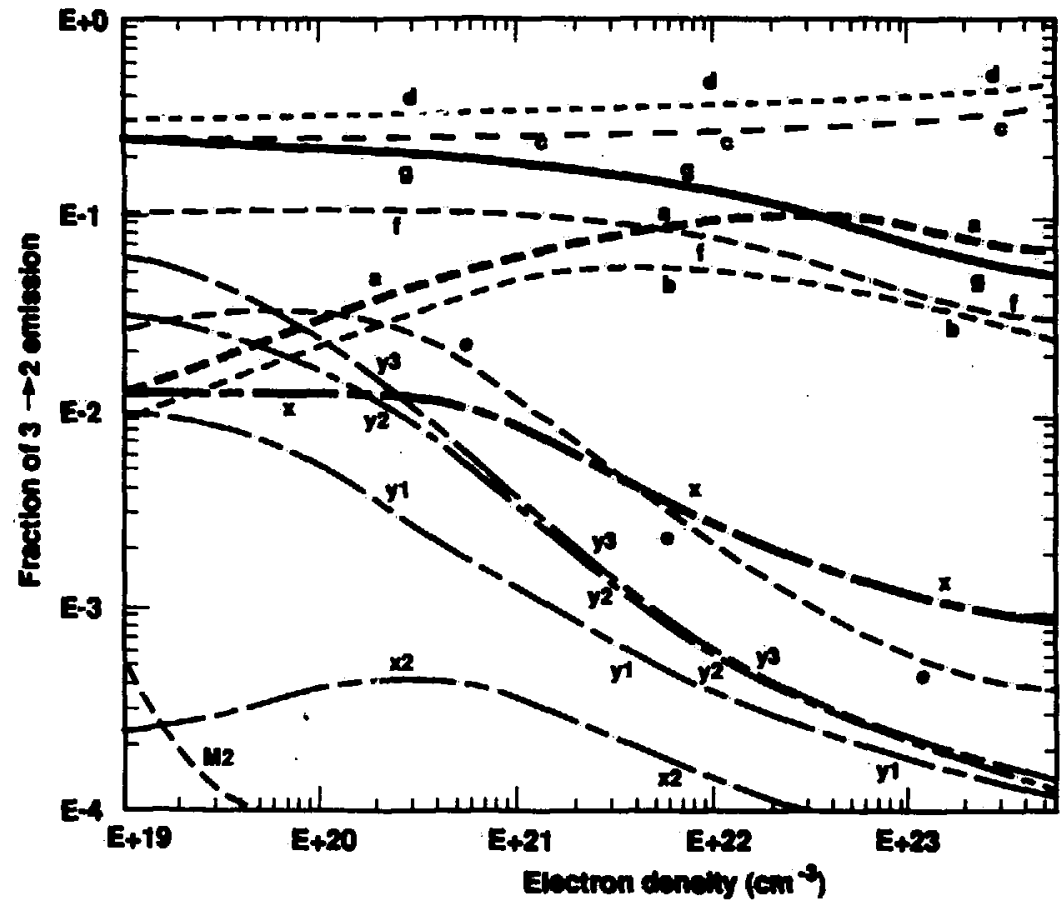

$\min _{10} \cos (R=4)$

$T 0=600$ or

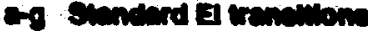

we ne

$x$ E2 (3d-uppor)

$\times 2$ E2 (adtowner)

y1 E2 (Bp-lipper)

y2 E2 (3p-minater)

y3 E2 (3p-lowner) 
primarily by collisional excitation from the Ne-like ground state. At a density of $10^{21} \mathrm{~cm}^{-3}, 67 \%$ of the flux into the " $\mathrm{x}$ " level comes from collisional excitation from the ground state. At $10^{21} \mathrm{~cm}^{-3}$, only $16 \%$ of the flux out of the "x" level appears as the " $x$ " electric quadrupole transition $\left(A_{\mathrm{x}}=3.1 \times 10^{11} \sec ^{-1}\right)$. $A$ large fraction of the flux out of the " $x$ " level is via collisional transitions to other excited levels, including about 32\% to the "3A" level. At lower densities, $10^{19} \mathrm{~cm}^{-3}$ (data not shown), 98\% of the " $x$ " level population comes from collisional excitation from the ground state. At $10^{19} \mathrm{~cm}^{-3}$, collisional transitions out of the " $\mathrm{x}$ " level are extremely small and the " $x$ " level population relaxes via electric dipole allowed transitions to other excited levels $\left\{18 \%\right.$ to the $\left[2 \mathrm{p}^{5} 3 \mathrm{~d}(1 / 2,5 / 2) \mathrm{J}=2\right]$ and $16 \%$ to the $\left[2 \mathrm{p}^{5} 3 \mathrm{~d}(1 / 2,5 / 2) \mathrm{J}=3\right]$ levels $\}$ as well as by electric quadrupole transitions to the Ne-like ground state (34\% to the ground state).

The "3A" (labeled as " $\mathrm{a}$ " in Figure 33.) resonance line intensity increases monotonically from $10^{19}$ to $10^{22} \mathrm{~cm}^{-3}$, reaching a maximum at about $3 \times 10^{22} \mathrm{~cm}^{-3}$, then decreasing very slowly at higher densities. From Table 5b, we see that at $10^{21}$ $\mathrm{cm}^{-3}, 11 \%$ of the "3A" population comes from collisional excitation from the ground state; $37 \%$ comes from collisional mixing with the $\left.\left[2 s 2 p^{6} 3 s(1 / 2,1 / 2)\right]=0\right]$ metastable level; $23 \%$ from the " $x$ " level; the remainder of the " $3 \mathrm{~A}$ " population comes from collisional transitions fron other excited metastable levels. Over 93\% of the "3A" level population relaxes via dipcle allowed radiative decay $\left(A_{3 A}=3.5 \times 10^{13} \sec ^{-1}\right)$ appearing as the "3A" Ne-likr resonance line. Therefore, the ratio between the " $\mathrm{x}$ " electric quadrupole line and the " $3 \mathrm{~A}$ " resonance line intensities are particularly sensitive io the electron density.

We should also note that the "3G" (labeled as " $g$ " in Figure 33.) decreases monotonically with density, very slowly between $10^{19}$ to $10^{22} \mathrm{~cm}^{-3}$, then slightly 
more rapid at higher densities as shown in Figure $5 \mathrm{c}$. At $10^{21} \mathrm{~cm}^{-3}$, the "3G" level is fed mostly by collisional transitions from the ground state and from the $2 s^{2} 2 p^{5} 3 p$ configuration. Over $85 \%$ of the "3G" level population radiatively decays to the ground state appearing as the " $3 G$ " Ne-like resonance line at $10^{21} \mathrm{~cm}^{-3}\left(\mathrm{~A}_{3 G}=1.1 \mathrm{x}\right.$ $\left.10^{13} \mathrm{sec}^{-1}\right)$. At higher densities $\left(\mathrm{n}_{\mathrm{e}} \geq 10^{22} \mathrm{~cm}^{-3}\right)$, collisional transition rates to other excited levels begin to effectively compete with the relatively strong dipole allowed redintive decay rate to the ground state.

A rough estimate of the " $x$ " line intensity can be made by assuming that the excited level is populated primarily by collisional excitation from the ground state. This should be valid for studying the " $x$ " level for densities below $10^{22} \mathrm{~cm}^{-3}$. The line intensity then becomes mostly dependent on the ground state population $\mathrm{N}_{\mathrm{g}}$, the electron density $n_{e}$, the excitation rate between the ground state and the excited level $\left\langle\sigma_{\mathrm{ij}}\right.$, and the "branching ratio" for the radiative decay from that excited level back down to the ground state $\omega_{\mathrm{ij}}$ as shown in the following expression:

$$
I_{i j}=N_{j} \cdot A_{i j}=N_{i} \cdot n_{e}\langle\nu \sigma\rangle_{i j} \cdot \omega_{i j} \text {, where } \omega_{i j}=\frac{A_{i j}}{\left.\sum_{k}\left(A_{k j}+n_{e}<v \sigma\right\rangle_{k j}\right)}
$$

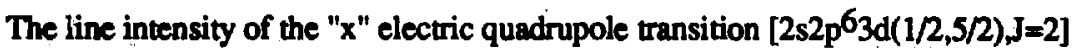
can be estimated by the expression:

$$
I_{x}=N_{g} \cdot n_{e}\langle v \sigma\rangle_{g \rightarrow x} \cdot \frac{A_{x}}{\left[A_{x}+n_{e} \cdot \sum_{i}\langle v \sigma\rangle_{i \leftrightarrow x}\right]}
$$

The "NELI" model transition rates for $\mathrm{Mo}^{32+}\left(500 \mathrm{eV}\right.$ and $\left.10^{21} \mathrm{~cm}^{-3}\right)$ can be used to estimate the density dependence of the "x", electric quadrupole line. At a density 
of $10^{21} \mathrm{~cm}^{-3}$, the electric quadrupole radiative decay rate $\left(A_{x}=3.1 \times 10^{11} \mathrm{sec}^{-1}\right)$ is smaller than both the total collisional transition rates into $\left(\mathrm{n}_{\mathrm{e}} \cdot \Sigma<v \sigma\right\rangle_{\text {in }}=1.06 \times 10^{12}$ $\left.\sec ^{-1}\right)$ and out of $\left(n_{e} \cdot \Sigma<v \sigma>_{\text {oul }}=9.62 \times 10^{11} \mathrm{sec}^{-1}\right)$ the $\left.\left[2 \mathrm{~s} 2 \mathrm{p}^{6} \mathrm{dd}(1 / 2,5 / 2)\right]=2\right]$ excited state of the " $x$ " transition. Therefore, the excited state population of the " $x$ " quadrupole transition will be significantly affected by collisional processes at densities near $10^{21} \mathrm{~cm}^{-3}$. The "critical" density where collisional deexcitations effectively compete with radiative decays can be estimated by comparing the radiative decay rate to the total collisional rate out of the excited level:

$$
\mathrm{n}_{e}^{\text {crit }}(\mathrm{x})=\frac{\mathrm{A}_{\mathrm{x}}}{\sum_{\mathrm{i}}\langle U \sigma\rangle_{\mathrm{i} \leftrightarrow \mathrm{x}}}=\frac{3.1 \times 10^{11} \mathrm{sec}^{-1}}{9.62 \times 10^{-10} \mathrm{sec}^{-1} \cdot \mathrm{cm}^{3}}=3.2 \times 10^{20} \mathrm{~cm}^{-3}
$$

We can see that for electron densities above $3.2 \times 10^{20} \mathrm{~cm}^{-3}$, the " $\mathrm{x}$ " to " $3 \mathrm{~A}^{\text {" line }}$ intensity ratio decreases with increasing electron density as predicted by the "NELI" calculation shown in Figure 33. In fact, the "NELI" model shows that at $10^{21} \mathrm{~cm}^{-3}$, $32 \%$ of the " $x$ " level population is directly (collisionally) transferred to the " $3 \mathrm{~A}$ " level. [Table 5a.] We see that even for densities below this value $\left(n_{e} \geq 10^{19} \mathrm{~cm}^{-3}\right)$, the " $x "$ to" $3 A$ " line intensity ratio falls off with density. This is primarily due to the density sensitivity of the " $3 \mathrm{~A}$ " level as described earlier in this section. This predicted density dependence of the " $x / 3 A$ " line intensity ratio is tested experimentally in Chapter 6.

The line intensity ratios between the " $x$ " electric quadrupole transition $\left[2 s^{2} p^{6} 3 d(1 / 2,5 / 2) J=2\right]-\left[2 s^{2} 2 p^{6}(0,0) J=0\right]$ and the dipole allowed "3A" $\left[2 s^{2} p^{6} 3 p(1 / 2,3 / 2) J=1\right]-\left[2 s^{2} 2 p^{6}(0,0) J=0\right] x$-ray transition are presented as a function of the electron density for electron temperatures of 350,500 , and $850 \mathrm{eV}$ in 
Figures 34(left) and 34(right), for Mo and Ag, respectively. The " $x / 3 A$ " line ratio appears to be only slightly temperature dependent and primarily at low temperatures. The line intensity ratio between the " $3 G^{\prime \prime}\left[2 s^{2} 2 p^{5} 3 s(1 / 2,1 / 2) J=1\right]-\left[2 s^{2} 2 p^{6}(0,0) J=0\right]$ transition and the " $3 \mathrm{~A}$ " line is plotted as a function of the electron density for electron temperatures of 500, 1000, $1 \mathrm{~d} 1500 \mathrm{eV}$ in Figures 35(left) and 35(right) for Mo and $\mathrm{Ag}$, respectively. As shown in Figure 35, this ratio "3G/3A" varies more slowly with density than the " $x / 3 A$ " ratio. While the " $3 A$ " line intensity is affected by collisional processes, the " $3 G$ " level lies further away (more isolated) from adjacent "metastable" levels and is less sensitive to density variations. This is unlike the " $x / 3 A$ " line intensity ratio in which both excited levels are density sensitive. Also, the "3G/3A" line intensity ratio will be more sensitive to radiative cascades which feed the lower lying "3G" level and therefore this line ratio is also more temperature sensitive. Both the " $\mathrm{x} / 3 \mathrm{~A}$ " and the " $3 \mathrm{G} / 3 \mathrm{~A}$ " line intensity ratios are predicted to be density sensitive by the "NELI" collisional radiative code and the more detailed steady state, 89 level Ne-like model described in the following section.

\subsubsection{Steady State, 89 Level Ne-Like Collisional Radiative Model}

A second, more detailed model developed by W. Goldstein and A. Osterheld (1988) has also been used to test the density sensitive nature of these Ne-like electric dipole and quadrupole lines. This Ne-like model is similar to the quasi-steady state K-shell model described in section 4.1.1. The steady state Ne-like collisional radiative model includes the Ne-like ground state $\left[2 s^{2} 2 p^{6}(0,0) \mathrm{J}=0\right]$ and the 88 lowest lying $n=3$ and $n=4$ singly excited states. The level por ulations are calculated from a detailed balance between radiative decay rates and electron collision rates. The model assumes an optically thin plasma and considers only Ne-like ions 
Figure 34. The density dependence of the Ne-like electric quadrupole to resonance line ratio, "x/3A", piedicted by the 37 level "NELI" model are shown for temperatures of 350, 500, and $850 \mathrm{eV}$ for (left) $\mathrm{Mo}(\mathrm{Z}=42)$ and (right) $\mathrm{Ag}(\mathrm{Z}=47)$. 
Figure 34

$z=42(\mathrm{Ho})$
$n i=1.000+1 \mathrm{C}^{\circ}$

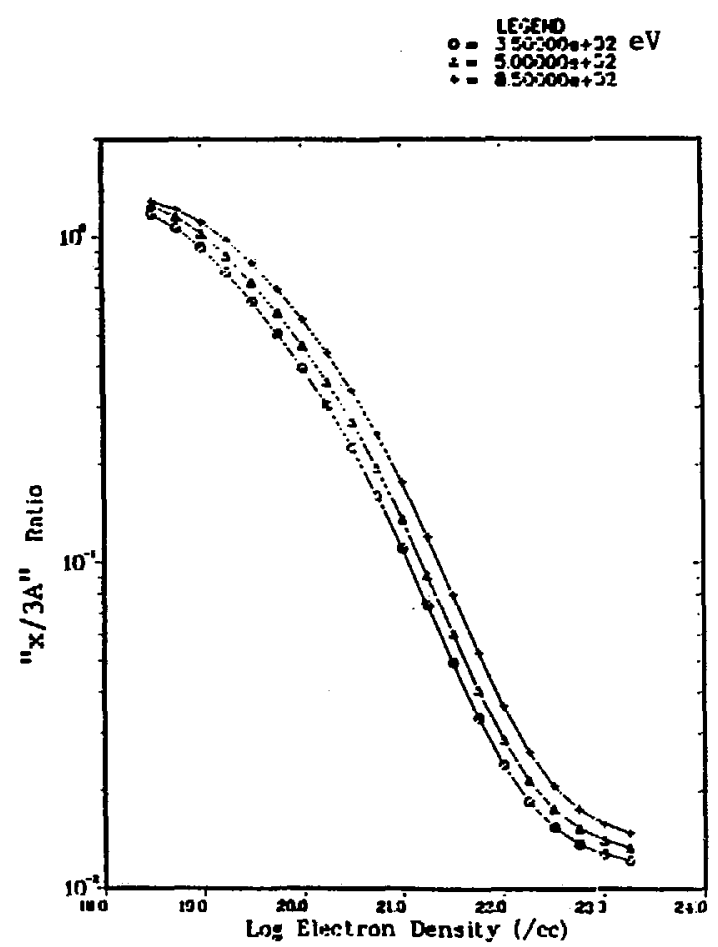

$$
\begin{aligned}
& z=47(\mathrm{Ag}) \\
& n i=1.000 * 10^{\circ}
\end{aligned}
$$

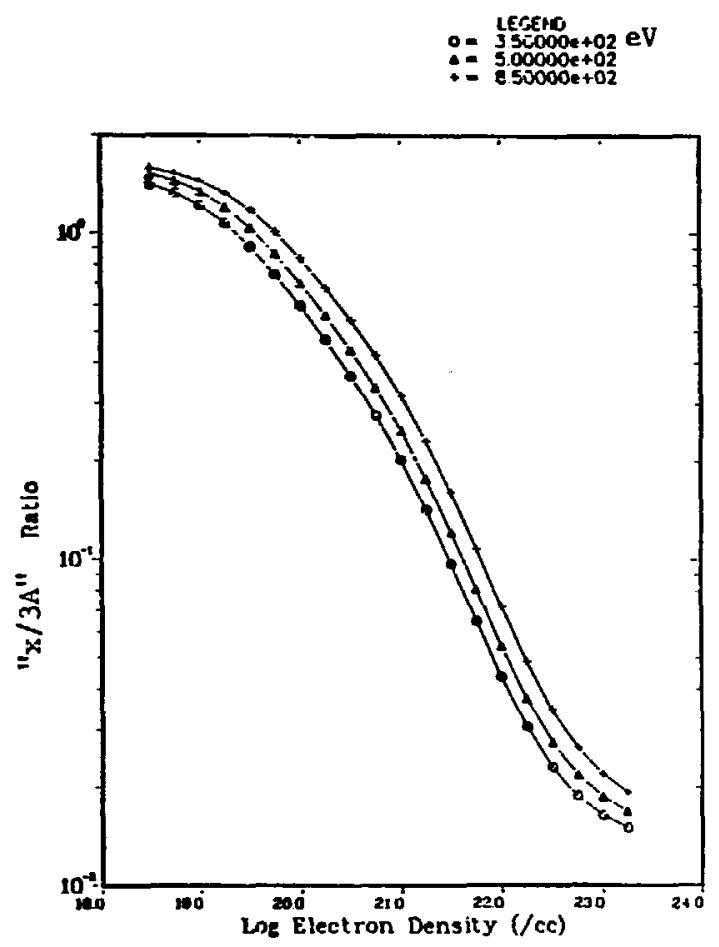


$-183-$

Figure 35. The density dependence of the Ne-like resonance line ratio, "3G/3A", predicted by the 37 level "NELI" model are shown for temperatures of 350,500 , and $850 \mathrm{eV}$ for (left) $\mathrm{Mo}(\mathrm{Z}=42)$ and (right) $\mathrm{Ag}(\mathrm{Z}=47)$. 


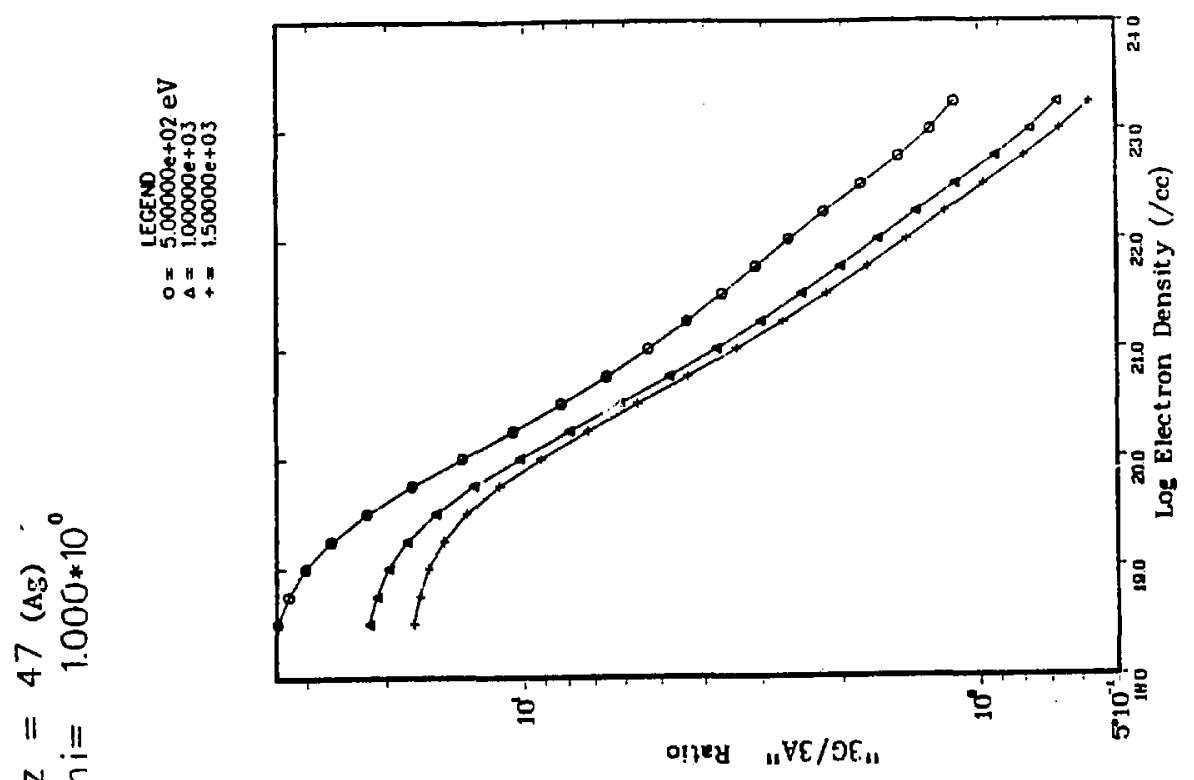

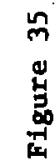

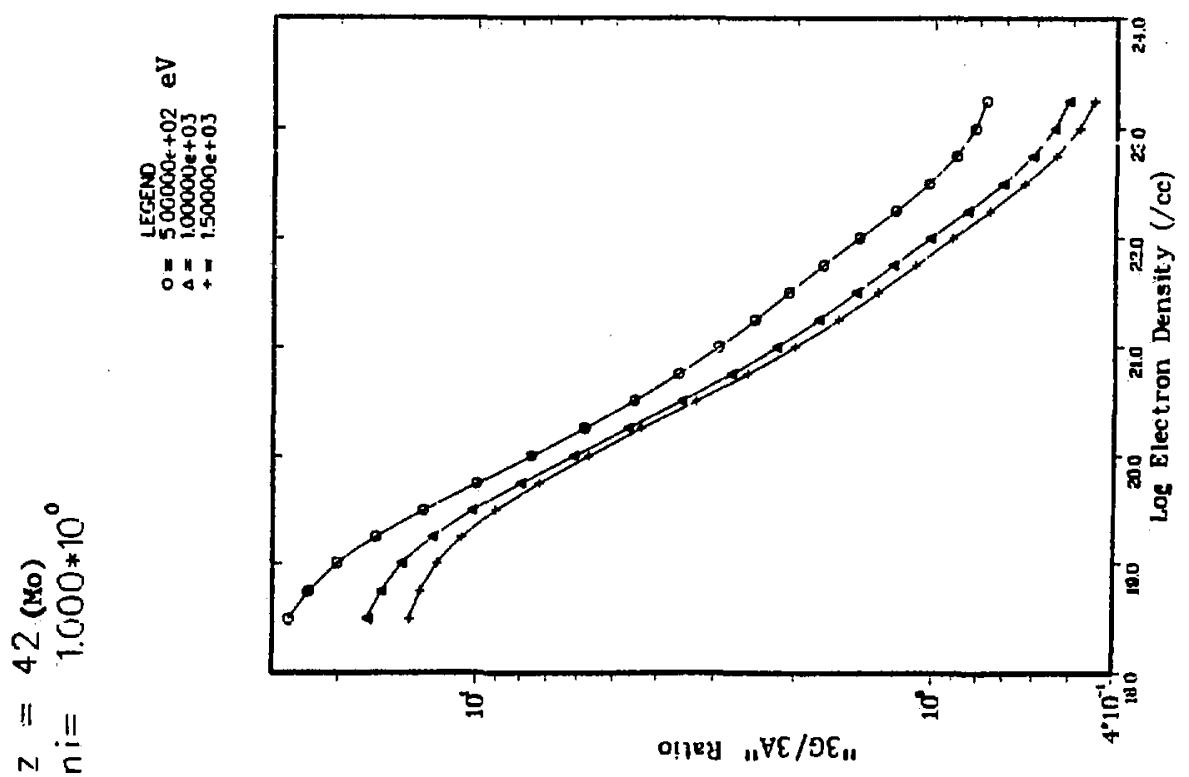


neglecting all ionization and recombination processes. A more detailed "quasi-steady state" L-shell collisional radiative model is currently in preparation. The complete model will allow for the detailed study of the quasi-steady state L-shell system, including F-like, Ne-like, and $\mathrm{Na}$-like ions. The relative level populations will be solved for by independently adjusting the relative charge state fractions (treated as free parameters) and their corresponding recombination and ionization contributions. For this analysis, only the 89 lowest lying Ne-like levels and direct excitation contributions are considered.

This 89 level mudel differs from the 37 level "NELI" model in a variety of ways. First, the excited states corresponding to singly excited $n=3$ and $n=4$ states are included. Radiative cascades from the $n=4$ to the $n=3$ levels as well as collisional transitions between these excited states are fully accounted for. All energy levels as well as radiative and collisional rates (DWA-distorted wave approximation) were computed using the Klapisch-Bar-Shalom codes which are based on Klapisch's relativistic parametric central potential method. [Klapisch (1971; 1977); Bar-Shalom et al.(1987)] The typical output is quite complete and too bulky to present in this thesis. It lists the relative line intensities for each electric dipole and quadrupole transition, as well as the radiative and collisional flux rates in and out of each $(n=3,4)$ excited level. Instead, the calculations for both molybdenum and silver, at a temperature of $500 \mathrm{eV}$ and a range of densities between $5.0 \times 10^{18}$ and $1.0 \times 10^{23}$ $\mathrm{cm}^{-3}$ are presented in Figure 36. Here the " $x / 3 \mathrm{~A}$ " line intensity ratio predicted by the 89 level model for Mo and Ag are plotted against the 37 level "NELI" model predictions in Figures 36(top) and 36(bottom), respectively. These line intensity ratios agree to within $10 \%$ with slight differences between the simpler "NELI" code predictions and the more detailed model and only at densities below $5 \times 10^{19} \mathrm{~cm}^{-3}$ and above $5 \times 10^{22} \mathrm{~cm}^{-3}$. This figure indicates that collisional mixing between the 
Figure 36. A comparison of the Ne-like electric quadrupole to resonance line ratio, "x/3A", predicted by the 37 level "NELI" model $(350,500$ and $850 \mathrm{eV})$ and by a more detailed 89 level model ( $500 \mathrm{eV}$ ) for (top) $\mathrm{Mo}(\mathrm{Z}=42)$ and (bottom) $\mathrm{Ag}(\mathrm{Z}=47)$. The model predictions differ only for densities below $5 \times 10^{19} \mathrm{~cm}^{-3}$ and higher than $5 \times 10^{22} \mathrm{~cm}^{-3}$. 

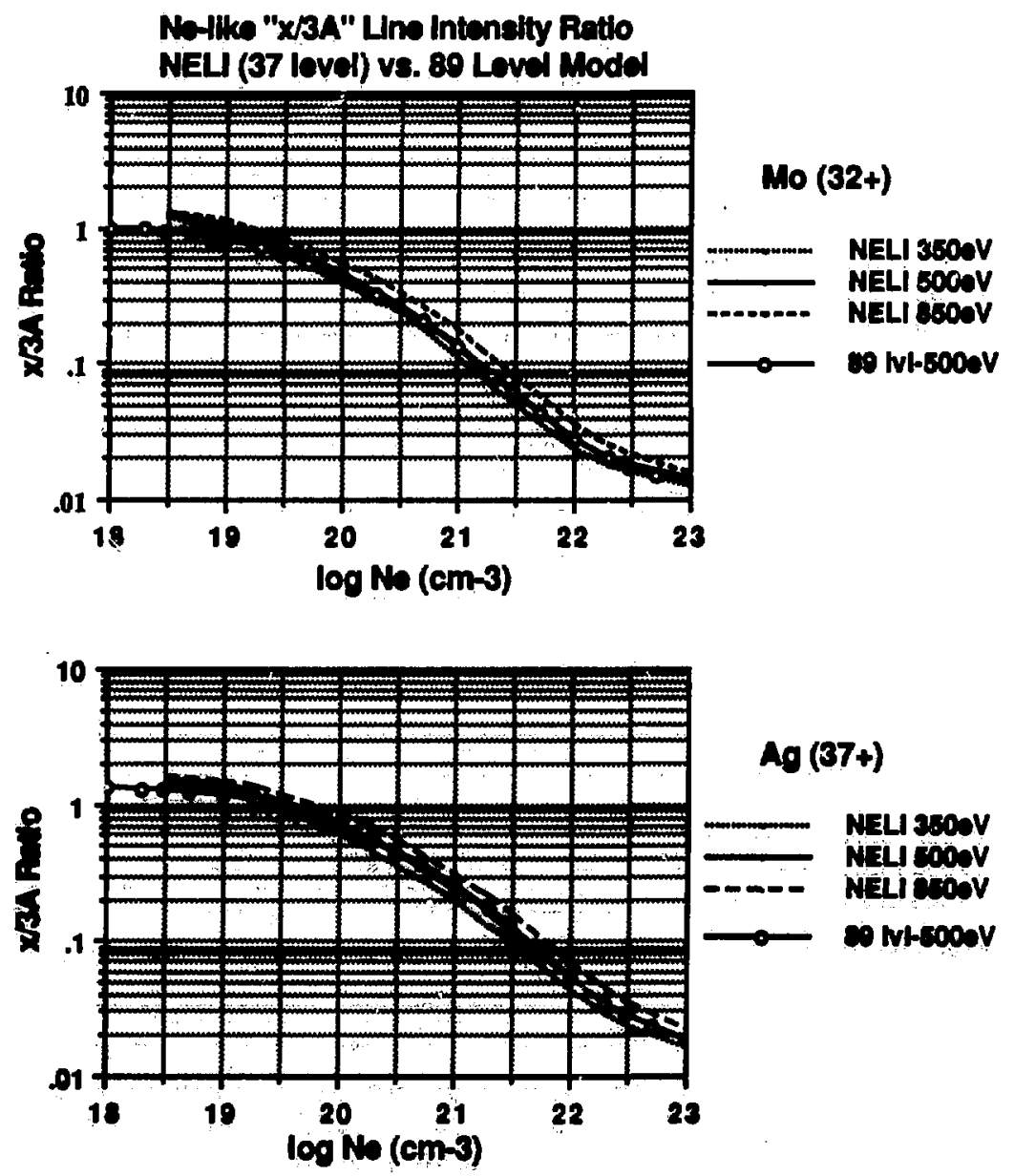

Figure 36 
excited $n=3$ and the $n=4$ levels are small and that the simpler "NELI" model is sufficiently detailed in this density region to study the density dependence of the " $x / 3 A$ " line intensity ratio. Since collisional mixing and radiative cascade processes appear to be significant population paths for the metastable levels, it is important that recombination and iorization processes be examined in future work. This 89 level Ne-like model is used to estimate gains for potential short wavelength lasing transitions which are presented in a discussion in Chapter 6.

\subsubsection{Recombination and Ionization Effects on the Electric Quadrupole and Rezonance Lines in Ne-like Mo32+}

A more detailed, quasi-steady state $\mathrm{Ne}-$ like $\mathrm{Mo}^{32+}$ calculation has recently been performed by A. Osterheld of LLNL (1988). The "quasi-steady state" model is based on the 89 level Ne-like system described in the previous section and was used to estimate the effects of recombination from $\mathrm{F}$-like on the $\mathrm{Ne}$-like $\mathrm{Mo}^{32+}$ level populations. The F-like ground state consists of three ground stares: $\left[1 s^{2} 2 s^{2} 2 p^{5}(0,3 / 2) J=3 / 2\right],\left[1 s^{2} 2 s^{2} 2 p^{5}(0,1 / 2) J=1 / 2\right]$, and $\left.\left[1 s^{2} 2 s 2 p^{6}(1 / 2,0)\right]=1 / 2\right]$. In Table 6, I have listed the various recombination rate coefficients computed from the Klapisch-Bar-Shalom codes feeding the " $x$ ", "3A", and "3G" Ne-like excited state levels for temperatures of 300 and $500 \mathrm{eV}$. [Klapisch (1971; 1977); Bar-Shalom at al.(1987)] I bave also listed the inner-shell ionization rate coefficients from Na-like ions to these levels in Table 6. Ionization effects have not been addressed at this time but at these low temperatures $\left(T_{e} \leq 500 \mathrm{ev}\right)$, the inner-shell ionization rates will be relatively small and should not affect this line ratio significantly.

Dielectronic recombination (as well as radiative and 3-body recombination) can be 
Table 6. The recombination rate coefficients (including radiative, 3-body, and dielectronic) from the F-like ground states and collision:! (inner shell) ionization rates coefficients from the Na-like singly excited states to the $\mathrm{Mo}^{32+}$ " $\mathrm{x}$ ", "3A", and "3G" excited state levels have been calculated using the the quasi-steady state, 89 ievel (Ne-like) L-shell model for electron temperatures of $300 \mathrm{eV}$ and $500 \mathrm{eV}$. 


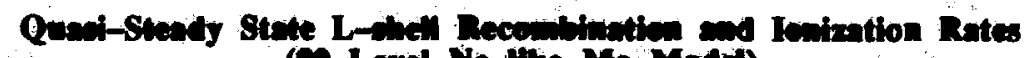
(8s-Level Ne-ithe MS Madi)

\begin{tabular}{|c|c|c|c|c|c|c|}
\hline Rne Creficions & $2 s^{2} 2 \mathrm{p} 53$ & $\frac{J^{n}}{2,1 / 2) J=1}$ & $282 \mathrm{p} 6 \mathrm{Bp}_{\mathrm{p}}(1)$ & $3 / 2) \mathrm{J}=1$ & $2 s 2 p^{63 d}(1)$ & $, 5 / 2) \mathrm{J}=2$ \\
\hline & $300 \mathrm{eV}$ & $500 \mathrm{eV}$ & $300 \mathrm{eV}$ & $500 \mathrm{eV}$ & $300 \mathrm{eV}$ & $500 \mathrm{eV}$ \\
\hline $\begin{array}{l}\text { Brimive Decotih, } \\
2 s^{2} 2 \mathrm{p}^{5}(0,3 / 2) \mathrm{J}=3 / 2 \\
2 \mathrm{~s}^{2} 2 \mathrm{p}^{5}(0,1 / 2) \mathrm{J}=1 / 2 \\
2 \mathrm{~s}^{2} \mathrm{p}^{6}(1 / 2,0) \mathrm{J}=1 / 2\end{array}$ & $1.966 \mathrm{e}-13$ & $1.499 e-13$ & $4.372 e-13$ & $3.261 e-13$ & $4.677 e-13$ & $3.263 e-13$ \\
\hline $\begin{array}{l}\text { 3-Body Recomh } \\
2 s^{2} 2 p^{5}(0,3 / 2) J=3 / 2 \\
2 s^{2} 2 p^{5}(0,1 / 2) J=1 / 2 \\
2 s 2 p^{6}(1 / 2,0) J=1 / 2\end{array}$ & $3.033 e-37$ & $1.699-37$ & $6.708 e-37$ & $3.748 c-37$ & $1.235 e-36$ & $6.885 e-37$ \\
\hline $\begin{array}{l}\text { Dielectronic Becsmb. } \\
2 s^{2} 2 p^{5}(0,3 / 2) J=3 / 2 \\
2 s^{2} 2 p^{5}(0,1 / 2) J=1 / 2 \\
2 s^{6} p^{6}(1 / 2,0) J=1 / 2\end{array}$ & $\begin{array}{l}6.120 e-13 \\
4.710 e-13 \\
5.550 e-14\end{array}$ & $\begin{array}{l}8.300 e-13 \\
6.060 c-13 \\
6.110 e-14\end{array}$ & $\begin{array}{l}1.770 e-14 \\
7.440 e-14 \\
1.030 e-12\end{array}$ & $\begin{array}{l}3.250 e-14 \\
1.330 e-13 \\
1.580 e-12\end{array}$ & $\begin{array}{l}2.450 e-14 \\
7.270 e-14 \\
1.110 e-12\end{array}$ & $\begin{array}{l}5.560 \mathrm{e}-14 \\
1.560 \mathrm{e}-13 \\
1.910 \mathrm{e}-12\end{array}$ \\
\hline $\begin{array}{l}\text { Collisionsl Joniztion } \\
2 s^{2} 2 p^{6} 3 s(0,1 / 2) J=1 / 2 \\
2 s^{2} 2 p^{6} 3 p(0,3 / 2) J=3 / 2 \\
2 s^{2} 2 p^{6} 3 d(0,5 / 2) J=5 / 2\end{array}$ & $1.234 e-17$ & $1.911 \mathrm{e}-15$ & $1.058 e-18$ & $2.286 e-16$ & $1.074 e-18$ & $2.308 e-16$ \\
\hline
\end{tabular}


an important populating mechanism for these Ne-like transitions. In Figure 37, the Ne-like $\mathrm{Mo}^{32+}$ " $\mathrm{x} / 3 \mathrm{~A}$ " and " $3 \mathrm{G} / 3 \mathrm{~A}$ " line intensity ratios have been plotted versus the electron density for both the direct excitation case (Ne-like only) and for the purely recombining plasma case (89 level Ne-like system plus the three F-like ground states) for a temperature of $500 \mathrm{eV}$. These calculations are very similar to the recombination and direct excitation calculations performed on the AI K-shell system described in section 4.1.1. For the recombining Ne-like $\mathrm{Mo}^{32+}$ plasma, the Ne-like ground state population was initially set to zero so that the initial Ne-like excited state population originated entirely by recombination from the F-like ground states, then subsequently redistribured by collisional mixing and radintive cascades. For the " $x / 3 A^{\prime}$ line ratio [Figure 37(top)], the recombination calculation predicts much lower line invensity ratios than for the pure direct excitation case for densities below $2 \mathrm{x}$ $10^{20} \mathrm{~cm}^{-3}$. For the purely excitation case, the " $x$ " excited state level receives most of its population from direct excitar in from the Ne-like ground state as described in section 4.3.2. [Sec Table 5.] In the recombining plasma, both the " $x$ " and "3A" levels are populated similarly (via recombination processes) therefore the " $x / 3 A$ " line intensity ratio shows no (or little) density variation for densities below $2 \times 10^{20}$ $\mathrm{cm}^{-3}$. For higher densities, collisional mixing between the excited state level populations produce approximately the same density dependence as predicted for the pure excitation case. Therefore, for densities above $2 \times 10^{20} \mathrm{~cm}^{-3}$, the " $x / 3 \mathrm{~A}^{\prime}$ line intensity should be density dependent as predicted by either model. Above $2 \times 10^{20}$ $\mathrm{cm}^{-3}$, the density dependence of the " $\mathrm{x} / 3 \mathrm{~A}$ " line intensity ratio will be relatively independent of the primary atomic processes governing the population kinetics of the ions. This is most fortunate since the L-shell experiments conducted in this work and presented in Chapter 6, focussed on the density region between $10^{20}-10^{21} \mathrm{~cm}^{-3}$. The " $3 \mathrm{G} / 3 \mathrm{~A}$ " line intensity ratio is affected much more dramatically as shown in 
Figure 37. The " $x / 3 A$ " and " $3 G / 3 A$ " line intensity ratios as a function of the electron density are predicted for a purely direct excitation plasma and for a purely recombining plasma using the 89 (Ne-like) level quasi-steady state Mo L-shell model based on a temperature of $500 \mathrm{eV}$. 
89-Lovel No-like Mo at 500 oV

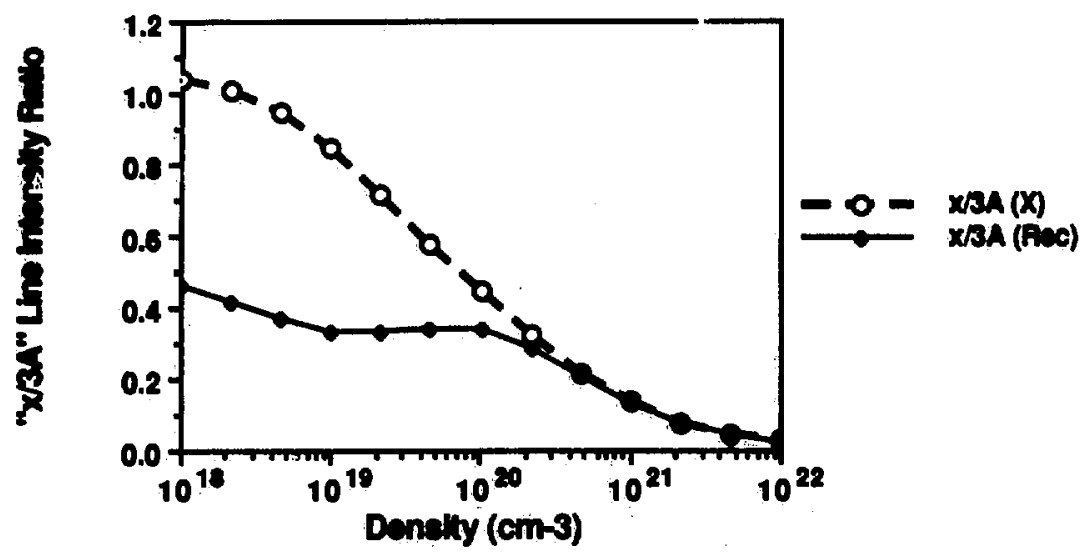

89-Lovel No-like No at 500 oV

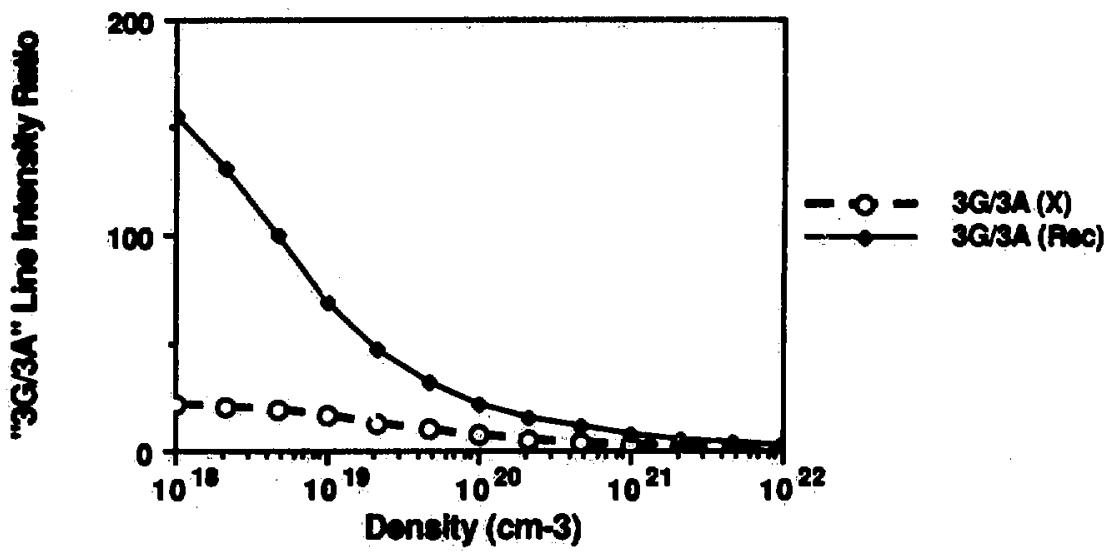

Figure 37 
Figure 37(bottom). Because the "3G" excited state is a relatively low lying level, it tends to be fed by collisions and cascades from higher lying levels. In a recombining plasma, a majority of the upper excited levels get populated via recombination processes (more than via direct excitation from the ground Ne-like ground state). This large excited state population "filters" down through the low lying " $3 \mathrm{G}$ " level, thus increasing the " $3 \mathrm{G}$ " line intensity. Therefore, the predicted recombination "3G/3A" line intensity ratio is much higher than for the pure excitation case as shown in Figure 37(bottom).

Recombination processes will play an important role in determining the overnll Ne-like population kinetics and should be included in future theoretical atomic models used to study the Ne-like ions in laser-produced plasmas. Recombination clearly affects the "3G/3A" line intensity ratio for all densities. For densities below 2 $x 10^{20} \mathrm{~cm}^{-3}$, the " $x / 3 \mathrm{~A}^{\prime}$ line intensity ratio is also affected by recombination processes. For higher densities, the " $x$ " and " $3 A$ " excited state levels appear to be sufficiently collisionally coupled to each other and to other nearby metastable states so that the initial populating mechanism is not a significant factor. 


\section{Chapter 5. K-Shell Spectroscopy Experim?nts}

In this chapter, I describe the x-ray spectroscopy experiments conducted on laser-irradiated Al microdot targets. The simultaneously space and time resolved is reil spectra emitted by hydrogen-like, helium-like, and lithium-like ions have measured using the Framing Crystal X-ray Spectrometer (FCXS) and the X-ray Streaked Crystal Spectrograph (XSCS). These are the first spectroscopic measurements of laser-produced plasmas that are simultaneously resolved in time and in all three dimensions in space.

In particular, I have measured the density dependence of various $\mathrm{K}$-shell $\mathrm{x}$-ray emission lines over a wide range of laser-plasma conditions both during and after the heating laser pulse. The K-shell line ratios studied include the He-like $\left[1 \mathrm{~s}^{2}\left({ }^{1} \mathrm{~S}_{0}\right)-\right.$ $\left.1 \mathrm{~s} 2 \mathrm{p}\left({ }^{1} \mathrm{P}_{1}\right)\right]$ "He ${ }_{\alpha}$ " to He-like $\left[1 \mathrm{~s}^{2}\left({ }^{1} \mathrm{~S}_{0}\right)-1 \mathrm{~s} 2 \mathrm{p}\left({ }^{3} \mathrm{P}_{1}\right)\right]$ intercombination "IC" line ratio, the He-like $\left[1 s^{2}\left({ }^{1} S_{0}\right)-1 s^{3} p\left({ }^{1} P_{1}\right)\right]$ "Heß" to He-like $\left[1 s^{2}\left({ }^{1} S_{0}\right)-1 s 2 p\left({ }^{3} P_{1}\right)\right]$ intercombination "IC" line ratio, and the ratios between the high-n resonance lines in He-like ions such as the He-like $\left[1 s^{2}\left({ }^{1} S_{0}\right)-1 s 4 p\left({ }^{1} P_{1}\right)\right] ~ " H e \gamma " ~ t o ~ H e-l i k e ~\left[1 s^{2}\left({ }^{1} S_{0}\right)\right.$ $\left.-1 \mathrm{~s} 3 \mathrm{p}\left({ }^{1} \mathrm{P}_{1}\right)\right]$ "He$\beta "$ line ratio and the He-like $\left[1 \mathrm{~s}^{2}\left({ }^{1} \mathrm{~S}_{0}\right)-1 \mathrm{~s} 5 \mathrm{p}\left({ }^{1} \mathrm{P}_{1}\right)\right]$ "Hes" to He-like $\left[1 s^{2}\left({ }^{1} S_{0}\right)-1 s 3 p\left({ }^{1} P_{1}\right)\right]$ "He ${ }_{\beta}$ " resonance line ratio. These line ratios are density dependent due to collisional mixing between excited levels which effectively competes with the radiative decay rates out of these levels. Recombination processes which preferentially feed the high-n excited levels (especially the triplet states) in He-like ions are also important. The predicted density dependence of each of these line intensity ratios was described in Chapter 4. I have compared experimental line intensity ratios based on independently determined plasma conditions to theoretical atomic model predictions. 
In this chapter, a description of the $\mathrm{K}$-shell laser-produced microdot plasma experiments is presented in section 5.1. The basic goal of these experiments was the measurement of space and time resolved $\mathrm{K}$-shell line emission profiles from the $\mathrm{Al}$ microdot plasmas using the Framing Crystal X-ray Spectrometer (FCXS). The simultaneous space and time resolution provided a detailed picture of the spatial and temporal evolution of these hot dense plasmas and allowed for the clear comparisons of the density and temperature dependences of various measured $\mathrm{K}$-shell spectral line intensities to simple theoretical atomic model predictions. Accurate temperature profiles are required to fully chyacterize the laser-plasma and to clearly isolate the density dependence of the spectral lines studied. Therefore, the space and time resolved electron temperature profiles for $\mathrm{Al}$ microdot plasmas have, in most cases, been determined using the slope of the H-like free-bound recombination continuum measured with the FCXS. This provided a clean, (relatively) model independent temperature measurement. These measurements of the electron temperature profile are presented in section 5.2. I have also compared these new space and time resolved temperature and density profiles with detailed 2 dimensional hydrodynamic code simulations and find large discrepancies. These differences are discussed in section 5.2.1.

I have also determined the electron temperatures using standard line intensity ratio methods based on widely used CRE models. In particular, I used the He-like $1 s\left({ }^{1} S_{0}\right)-1 s 3 p\left({ }^{1} P_{1}\right)$ "He $\beta$ " to H-like $1 s-3 p$ "Ly ${ }_{\beta}$ " resonance line ratio and the H-like $1 s-3 p$ "Ly ${ }_{\beta}$ " to He-like $\left[1 s 2 p\left({ }^{1} P_{1}\right)-2 p^{2}\left({ }^{1} D_{2}\right)\right]$ dielectronic satellite line ratio. A brief review of the temperature dependence of these $\mathrm{K}$-shell line intensity ratios is presented in section 5.3. Measurements of the electron temperature profiles using these various CRE line intensity ratio techniques are described in section 5.4. During the laser heating pulse, I find significant differences between CRE resonance 
line ratio predicted temperatures and moderate agreement with the CRE satellite line ratio temperatures. Late in time after the heating laser is off, both CRE line ratio temperatures are far higher than the free-bound continuum values.

In section 5.5, I present the results of my measurements of the density sensitive "He $/ \mathrm{dC}$ " line ratio and comparisons to standard CRE model predictions. I find serious disagreement with standard Collisional Radiative Equilibrium (CRE) Model predictions. These differences are primarily due to the highly non-stationary ionization distribution characteristic to these laser-produced piusmas. The CRE models underestimates the recombination population paths feeding intercombination line $1 \mathrm{~s} 2 \mathrm{p}\left({ }^{3} \mathrm{P}_{1}\right)$ level and consistently underestimates the "IC" line intensity based on the CRE temperature and density. Also discussed are various experiments to minimize opacity effects on the measured line emission spectra including the use of a diluted, 10\% $\mathrm{Al}$ microdot target and the use of the " $\mathrm{He} / \mathrm{dC}$ "line intensity ratio. In section 5.5.1, my measurements are compared to the more detailed, quasi-steady state Al K-shell inodel predictions where I find yery good agreement for late times and plasma distances far out into the corona. The quasi-steady state model includes contributions from both collisional excitations from the He-like ground state as well as from recombination processes (3-body, radiative, and dielectronic) from the $H$-like ground state. Also, collisional mixing between excited states (including $\Delta \mathrm{n}=0$ collisions) and radiative cascades have been fully accounted for in the quasi-steady state model. In section 5.6, I present measurements of population inversions in the high-n [1 snp $\left.\left({ }^{1} \mathbf{P}_{1}\right)\right]$ metastable levels in $\mathrm{He-like} \mathrm{Al.} \mathrm{These} \mathrm{population} \mathrm{inversions} \mathrm{are}$ due to 3-body recombination and the subsequent redistribution of the excited state population among the metastable levels by collisional and radiative transitions. These population inversions are exhibited by the inverted resonance line intensity ratios measurements which have been correctly predicted by the quasi-steady state 
model. The space resolved, time-integrated spectra measurements for the same target shots have been analyzed using standard CRE model methods and are presented in section 5.7. This data represents the fairly poor quality of data typical of past laser-plasma experiments and clearly shows the shortcomings in previous attempts to characterize these high-energy, laser-produced plasmas. Finally in section 5.8, these new simultaneously space and time resolved measurements to probe localized plasmas and directly test the predicted density dependence of various K-shell line intensities are summarized.

\subsection{Description of the Experiments}

The experiments were conducted using the CHROMA laser facility at KMS Fusion, Inc. Simple planar microdot targets were irradiated with a single beam of $\mathbf{5 0}$ $\mathrm{J}$ of $526.0 \mathrm{~nm}$ light in a $1.0 \mathrm{~ns}$ wide stacked pulse (trapezoidal temporal profile with a 100 ps rise and fall time and a $1.0 \mathrm{~ns}$ flat top). The beam was focused (far field) onto the planar target surface to a diameter of $250 \mu \mathrm{m}$ using an $f / 2.5$ lens. The microdot targets were irradiated at approximately $1.0 \times 10^{14} \mathrm{~W} / \mathrm{cm}^{2}$. The majority of the targets studied in these experiments consisted of $100 \mu \mathrm{m}$ diameter, $>5 \mu \mathrm{m}$ thick microdots of $\mathrm{Al}$ mounted on a thick $(125 \mu \mathrm{m})$ polystyrene $(\mathrm{CH})_{\mathrm{x}}$ substrate. A second type of target consisted of a mixture of $\mathrm{Al}_{2} \mathrm{O}_{3}$ and graphite forming a $100 \mu \mathrm{m}$ diameter dot containing $10 \%$ (by number) Al. [The actual target was a pressed powder mixture consisting of $5 \cdot \mathrm{Al}_{2} \mathrm{O}_{3}+75 \cdot \mathrm{C}$.] The $10 \%$ Al microdot target was mounted on a $125 \mu \mathrm{m}$ thick polystyrene $(\mathrm{CH})_{x}$ substrate. These targets are more fully described in Chapter 2. The laser parameters used for each target analyzed in this work are presented in Table 7.

A wide array of plasma diagnostics were fielded to study the laser-produced 
Table 7. The heating laser parameters for target shots used in this work are listed including the shot number, brief target description, the energy on target (joules), the stacked laser pulse width (ps), the laser focal spot size ( $\mu$ m diameter), and the laser irradiance $\left(W / \mathrm{cm}^{2}\right)$. 
Tab1e 7

\section{Shot List}

\begin{tabular}{|c|c|c|c|c|c|}
\hline Shot* & Target Type & EOT(J) & FWHM & $\begin{array}{l}\text { Focal } \\
\text { Diameter }\end{array}$ & $\begin{array}{l}\text { Intensity } \\
\left(W / \mathrm{cm}^{2}\right)\end{array}$ \\
\hline 8433 & $100 \mathrm{\mu m} \mathrm{Al}(100 \%) \mathrm{dot}$ & 39.4 & $900 \mathrm{ps}$ & $250 \mu \mathrm{mD}$ & $-8.9 \times 10^{13}$ \\
\hline 8459 & $100 \mu \mathrm{m}$ Al (100\%) dot & 44.1 & 1011 & $250 \mu \mathrm{mD}$ & $-8.9 \times 10^{13}$ \\
\hline 8460 & $100 \mu \mathrm{m}$ Al (100\%) dot & 43.5 & 944 & $250 \mu \mathrm{mD}$ & $-9.4 \times 10^{13}$ \\
\hline 8464 & $100 \mathrm{um} \mathrm{Al}(100 \%) \mathrm{dot}$ & 47.8 & 950 & $250 \mu \mathrm{mD}$ & $-1.0 \times 10^{14}$ \\
\hline 8465 & $100 \mu \mathrm{m} \mathrm{Al}(100 \%)$ dot & 59.5 & 1000 & $250 \mu \mathrm{mD}$ & $=1.2 \times 10^{14}$ \\
\hline 8455 & $100 \mu \mathrm{m}$ Al (100\%) dot & 14.7 & 853 & $250 \mu \mathrm{mD}$ & $\approx 3.5 \times 10^{13}$ \\
\hline 8466 & $100 \mu \mathrm{m} A 1(100 \%) \mathrm{dot}$ & 18.8 & 949 & $250 \mu \mathrm{mD}$ & $-4.0 \times 10^{13}$ \\
\hline 8427 & $\begin{array}{c}1004 \mathrm{~m} \mathrm{Al}(10 \% \mathrm{Al}) \mathrm{dot} \\
\left(5 \cdot \mathrm{Al}_{2} \mathrm{O}_{3}+75 \cdot \mathrm{C}\right)\end{array}$ & 50.7 & 918 & $250 \mu \mathrm{mD}$ & $=1.1 \times 10^{14}$ \\
\hline 8502 & $\begin{array}{r}1: 8 \mathrm{Mo} / \mathrm{Mg} \text { bulis-eye } \\
(70 \mu \mathrm{m} / 170 \mu \mathrm{m})\end{array}$ & 148.8 & 942 & $250 \mu \mathrm{mD}$ & $-3.2 \times 10^{14}$ \\
\hline 8504 & $\begin{array}{r}1: 8 \mathrm{Mo} / \mathrm{Mg} \text { bulls-eye } \\
(70 \mu \mathrm{m} / 170 \mu \mathrm{m})\end{array}$ & 137.4 & 945 & $250 \mu \mathrm{mD}$ & $=3.0 \times 10^{14}$ \\
\hline 8515 & $100 \mu \mathrm{m}$ Ag $(100 \%)$ dot & 112.4 & 920 & $153 \mu \mathrm{mD}$ & $-6.7 \times 10^{14}$ \\
\hline 8517 & $100 \mu \mathrm{m} A g(100 \%) \mathrm{dot}$ & 118.6 & 940 & $153 \mu \mathrm{mD}$ & $-6.9 \times 10^{14}$ \\
\hline 8519 & $100 \mathrm{um} \mathrm{Ag}(100 \%) \mathrm{dot}$ & 116.3 & 900 & $200 \mu \mathrm{mD}$ & $-4.1 \times 10^{14}$ \\
\hline
\end{tabular}


plasmas including the FCXS, the XSCS, 2 space resolved, time-integrated flat crystal spectrographs, the four-frame holographic interferometer, and $2 \mathrm{x}$-ray pinhole cameras. The basic experimental set-up was șhown previously in Figure 7. A more detailed discussion of the space and time resolved instruments can be found in Chapter 2. Only the interferometry and x-ray spectroscopy data will be discussed in this chapter.

\subsubsection{XSCS Aluminum K-shell Data}

The X-ray Streaked Crystal Spectrometer (XSCS) provided a continuous recording of the K-shell emission spectra emitted from various $170 \mu \mathrm{m}$ wide regions of the plasma plume with approximately $30 \mathrm{ps}$ time resolution $(60 \mathrm{ps} / \mathrm{mm})$. Lifferent regions of the plasma plume were studied during similar target shots by adjusting the relative positions of the entrance and imaging slits. The XSCS spectra from a 100 $\mu \mathrm{m}$ diameter $\mathrm{Al}$ microdot target shot irradiated by $1.0 \times 10^{14} \mathrm{~W} / \mathrm{cm}^{2}$ was shown carlier in Figure 16. Two different plasma regions were studied: (a) Shot $\# 8433$ in which the XSCS viewed the plasma between the target surface and $150 \mu \mathrm{m}$ out into the plume (shown in Figure 16); and (b) Shot $\$ 8464$ in which the XSCS viewed the plasma between $150 \mu \mathrm{m}$ and $300 \mu \mathrm{m}$ from the target surface. It should be noted that the "He ${ }_{\alpha}$ " $\left[1 \mathrm{~s}^{2}-1 \mathrm{~s} 2 \mathrm{p}\left({ }^{1} \mathrm{P}_{1}\right)\right]$ resonance line appears to be occluded by part of the support structure for the imaging slit or by the end of the diffraction crystal, adversely altering its observed intensity. The remainder of the spectra is unperturbed and is representative of the recorded spectra from other typical Al dot target shots. A flat KAP diffraction crystal was used to measure the $\mathrm{Al} x$-ray emission from 5 to $8 \AA$ with a resolution of approximately 500 . The recorded images show the K-shell X-ray spectra diffracted off of the KAP crystal (horizontally) versus time (vertically) 
emitted by the defined region of the plasma plume. The XSCS data also reconded the CHROMA timing fiducial allowing for the comparison of data from different target șhots.

The XSCS dat clearly shows the rapidly evolving plasma conditions, both in time and space, indicated by the variations in the H-like and He-like resonance and satellite line intensities. Early during the laser heating pulse and for $150-300 \mu \mathrm{m}$ from the target surface, the emission is dominated by $\mathrm{H}$-like resonance lines and predominantly "Lyo" (1s-2p). The (1s-2p) to (1s-np) line intensity ratio does not vary as dramatically as seen in the corona of the plasma. Later in time, after the heating laser turns off (also further out into the plasm plume), the He-like resonance lines and the high-n H-like (1s-np) resonance lines appear strongly. At these late times, the (1s-2p) to (is-np) lire ratio changes significantly and is indicative of a cool, recombining plasma.

It is also interesting to nose that much of the resonance line emission, beyond 100 $\mu \mathrm{m}$ from the target substrate, occurs late in time after the heating laser is off. This behavior is more clearly seen in the sets of densitometer traces (representing Shots \#8433 and \#8464) in Figures 38a and 38b, respectively. These densitometer scans represent 60 patime intervals at various times during and after the laser heating pulse as indicated. Again, the reader is reminded to ignore the anomalous variation in the "Ly ${ }_{\alpha}$ " (1s-2p) line which is due to an obstruction in the XSCS field of view. The overall space and time history of the H-like and He-like resonance lines are aloo seen in the FCXS data and is clear evidence that previou: determinations of coronal plasma conditions based on time-integrated spectral data are likely in error. Near the target surface (near the critical density), the temporal and spatial gradients in the plasma are quite high. Experimental limitations, primarily in the finite spatial resolution $(-50 \mu \mathrm{m})$ and due to plasma opacity effects, prevent a clear determination 
Figure 38. Densitometer scans of XSCS spectra for two similar $100 \mu \mathrm{m}$ diameter aluminum target shots at $1.0 \times 10^{14} \mathrm{~W} / \mathrm{cm}^{2}$ for the plasma $\mathrm{r}$; ions a) Shot $\# 8433$ : from 0 to $150 \mu \mathrm{m}$, and b)Shot $\$ 8464$ : from 150 to $300 \mu \mathrm{m}$ from the target surface. In both sets of densitometer scans, thr. long wavelength region of the spectra seems to be occluded and the "He ${ }_{\alpha}$ " line intensity appears to be anomalous and should not be used. 
Shot: 2438
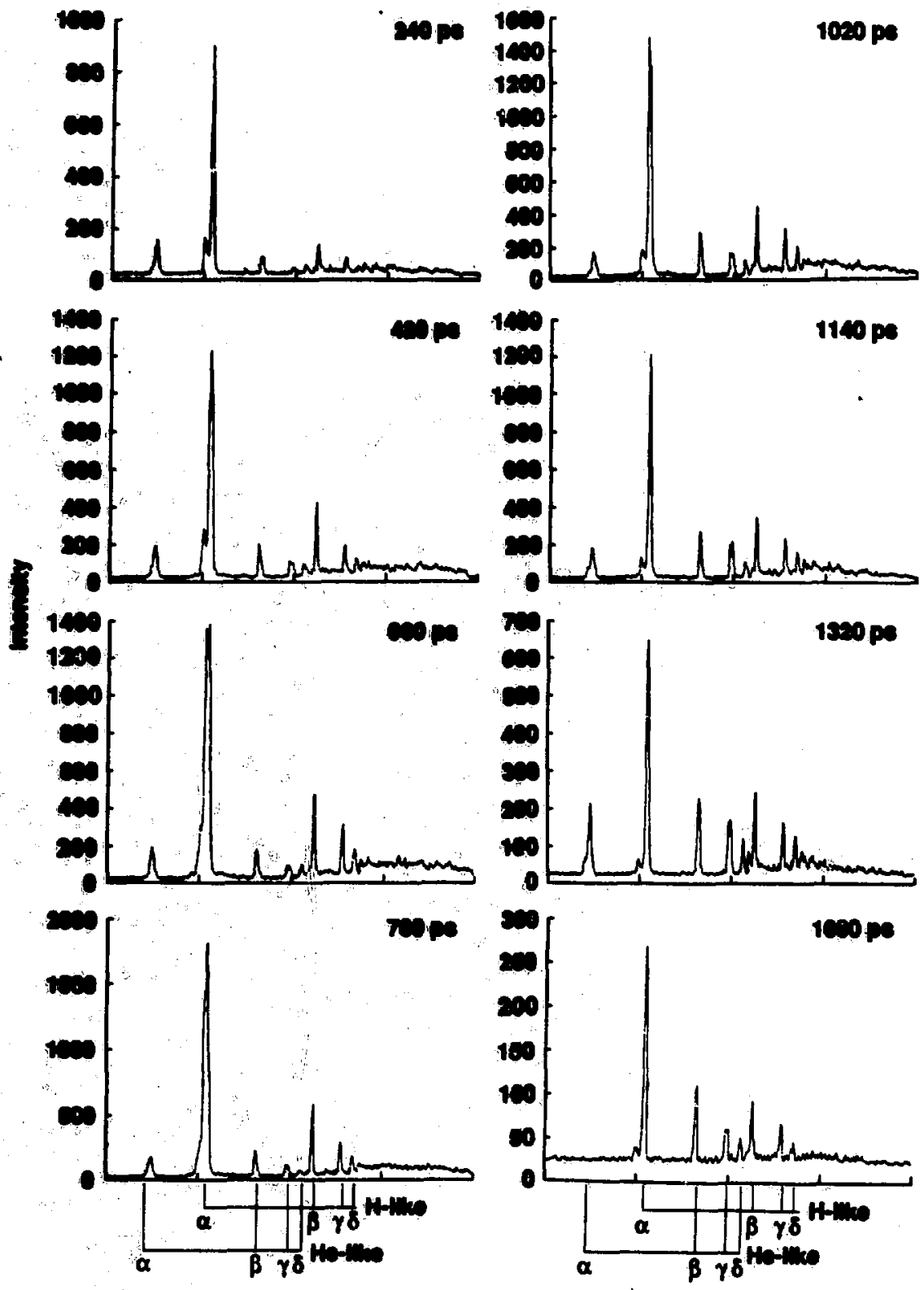

Figure $38 a$ 
Shon: 944

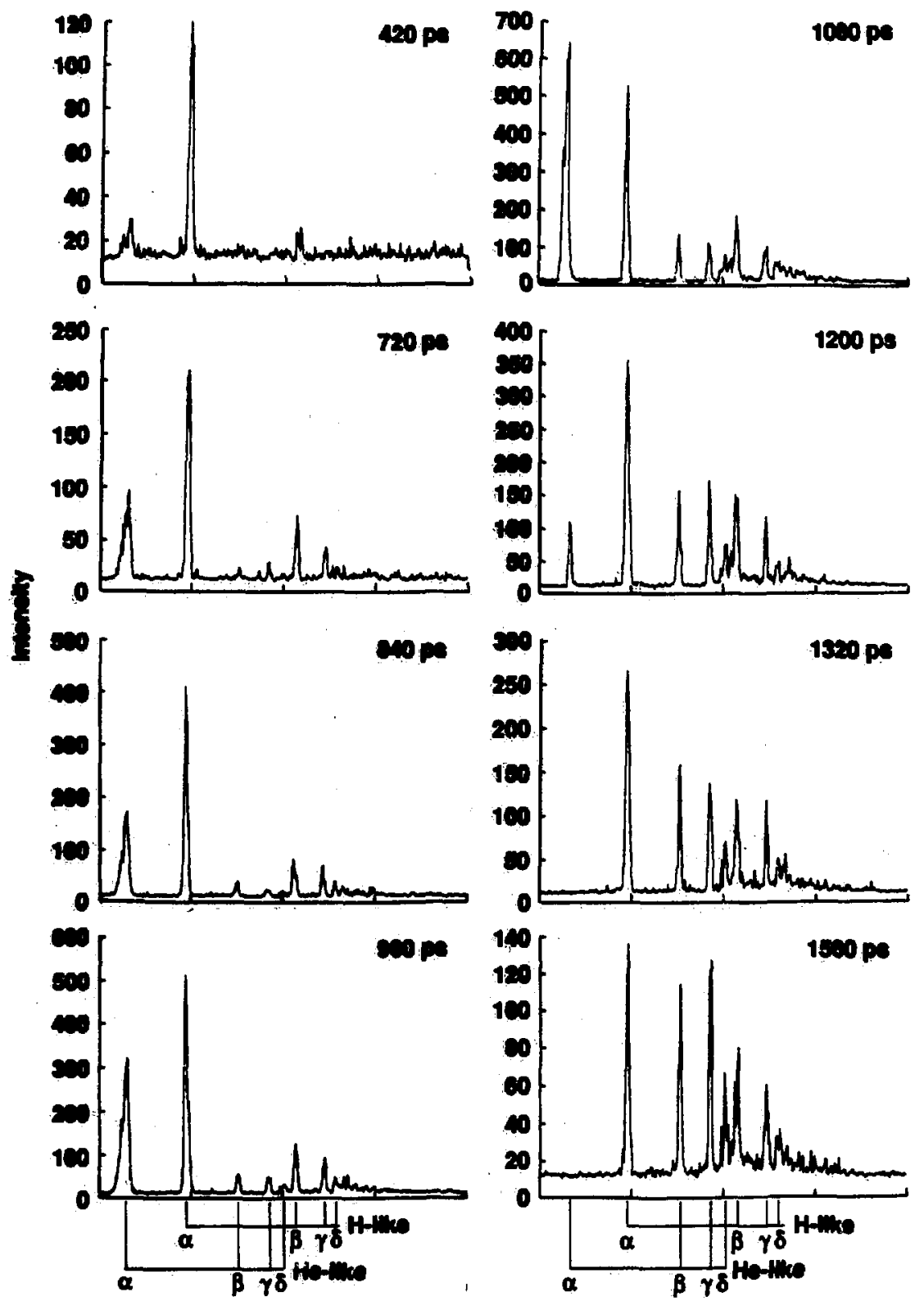


of these plasma conditions. Later is this chapter, I present space and time resolved mensurements of the electron temperature profile which provide, for the first time, reliable line intensity ratio based plasma diagnostics which accurately characterizes the evolving laser-produced plasma for all times and locations.

\subsubsection{FCXS Aluminum K-shell Date}

The Framing Crystal X-ray Spectrometer (FCXS) provided the bulk of the simultaneously space and time resolved $x$-ray emission spectral data used in the present work. For these experiments, the FCXS was operated using 250 ps wide time frames (cctual data frame times) and a spatial resolution of approximately 55 Hm. [See Chapver 2.] The FCXS used a flat KAP crystal to measure the Al K-shell spectra in the wavelength range of 4 to $8 \mathrm{~A}$ with a spectral resolution of approximacely 700 varying somewhat with wavelength. A set of framed spectra from a heated $\mathrm{Al}$ microdot torget $\left(1.0 \times 10^{14} \mathrm{~W} / \mathrm{cm}^{2}\right)$ was shown earlier in Figure 13 [Shot *8433]. Each strip represents the x-ray emission spectrum (film density) versus spatial extent (from the target surface out towards the incident heating laser beam) recorded from 1230-1480 ps (top strip), 730-980 ps (middle strip), and $1730-1980$ ps (bottom strip) with respect to the beginning of the 1.0 ns heating laser pulse.

Several similar Al microdot shots have been analyzed and provide a detailed picture of the evolving laser-plasma. The FCXS gain (MCP bias level) had to be increased to measure the relatively weak free-bound recombination continuum. When this was done, the measured emission lines were typically overexposed or saturated. Therefore, data from different target shots have been analyzed in this work, some measuring the free-bound continuum and some measuring line emission data. In some cases, two FCXS striplines were operated at the same gate time but at different 
gains (MCP bias voltages) and therefore, both line intensities and continuum measurements were determined from the same target shot. All targets shots presented in this analysis had nearly identical laser heating parameters and are listed in Table 7. The pulse widths of the shots varied by less than \pm 30 ps. The energy on target for these shots varied by less than \pm 3 joules and the irradiances of these shots were within $10 \%$ of ench other. All of the FCXS Al date presented in this chapter are for two laser irradiances: $=1.0 \times 10^{14} \mathrm{~W} / \mathrm{cm}^{2} \pm 10 \%$ (Shots $\# 8427$, $* 8433$, $\# 8459, \# 8460$, \#8464, and \#8465) and $-3.5 \times 10^{13} \mathrm{~W} / \mathrm{cm}^{2} \pm 10 \%$ (Shots $\# 8455$ and \#8466).

A series of densitometer scans representing the x-ray spectra emitted by $50 \mu \mathrm{m}$ wide regions of plasmu centered at various distances from the target surface for the time intervals of $730-980 \mathrm{ps}, 1230-1480$ ps $1730-1980$ ps are presented in Figures 39a. 39o, and 39c, respectively. [Shot \#8433 at $1.0 \times 10^{14} \mathrm{~W} / \mathrm{cm}^{2}$ ] The spectra clearly show dramatic spatial and temporal variations in the H-like and He-like resonance lines and indicates an ion distribution far from equilibrium as will be shown below. The dielectronic satellite lines to the H-like and He-like resonance lines are detectable at all times during the experiment (appearing on the long wavelength side of the resonance lines) and are strungest in intensity near the target surface, decreasing in intensity out into the plasma plume. This spatial gradient in the dielectronic satellite line has often seen in previous laser-plasma spectroscopy experiments. [For example: Boiko et al. (1977; 1984 ); Burkhalter et al. (1983); Bailey et al. (1986); Alaterre et al. (1984).] 
Figure 39. Densitometer scans of the FCXS data showing the aluminum K-shell radiation (Shot \#8433) emitted from $50 \mu \mathrm{m}$ wide regions of plasma at various distinces from the target surface for the time intervals a) $730-980 \mathrm{ps}$, b) $1230-1480$ ps, and c) $1730-1980 \mathrm{ps}$. 


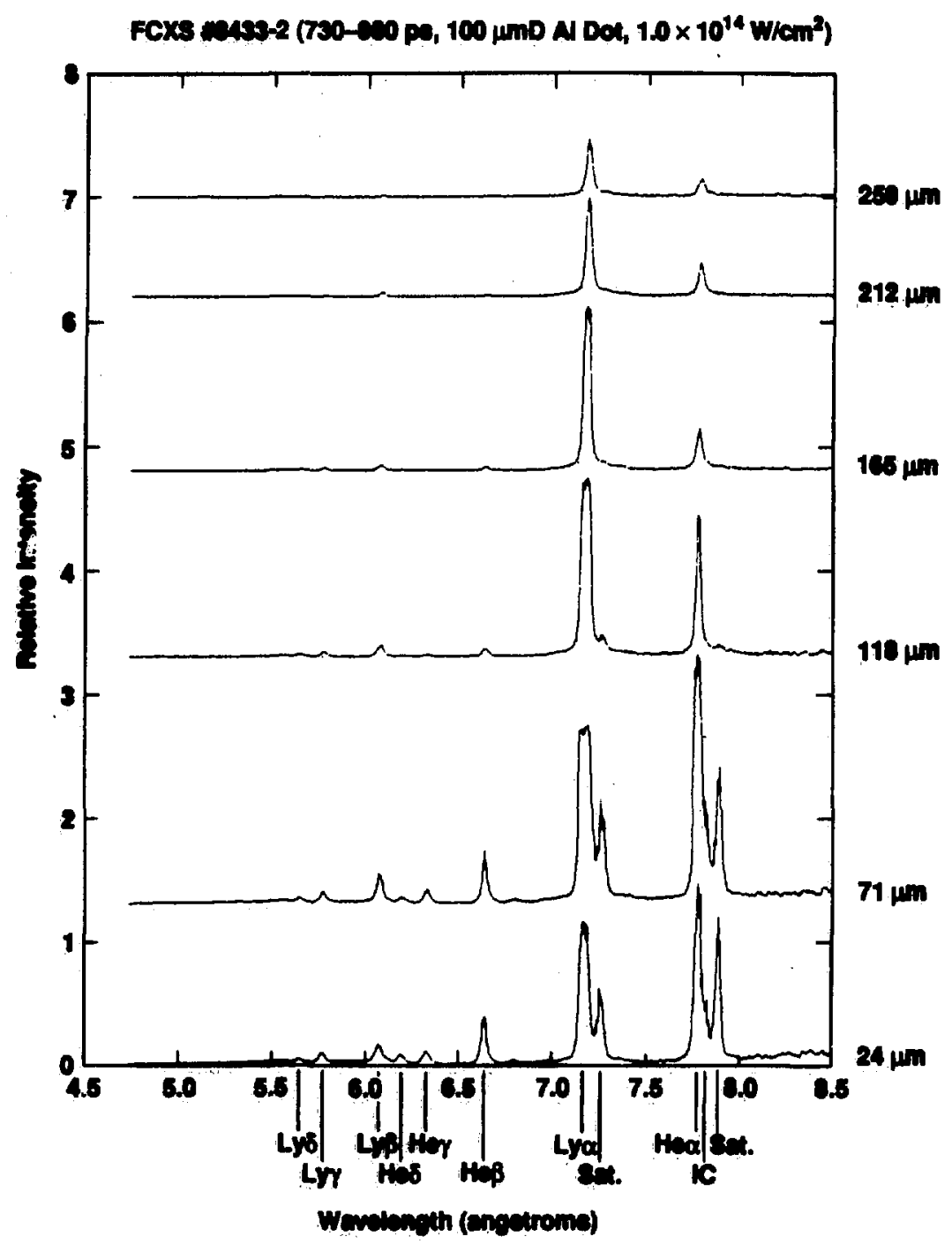

Figure $39 a$ 


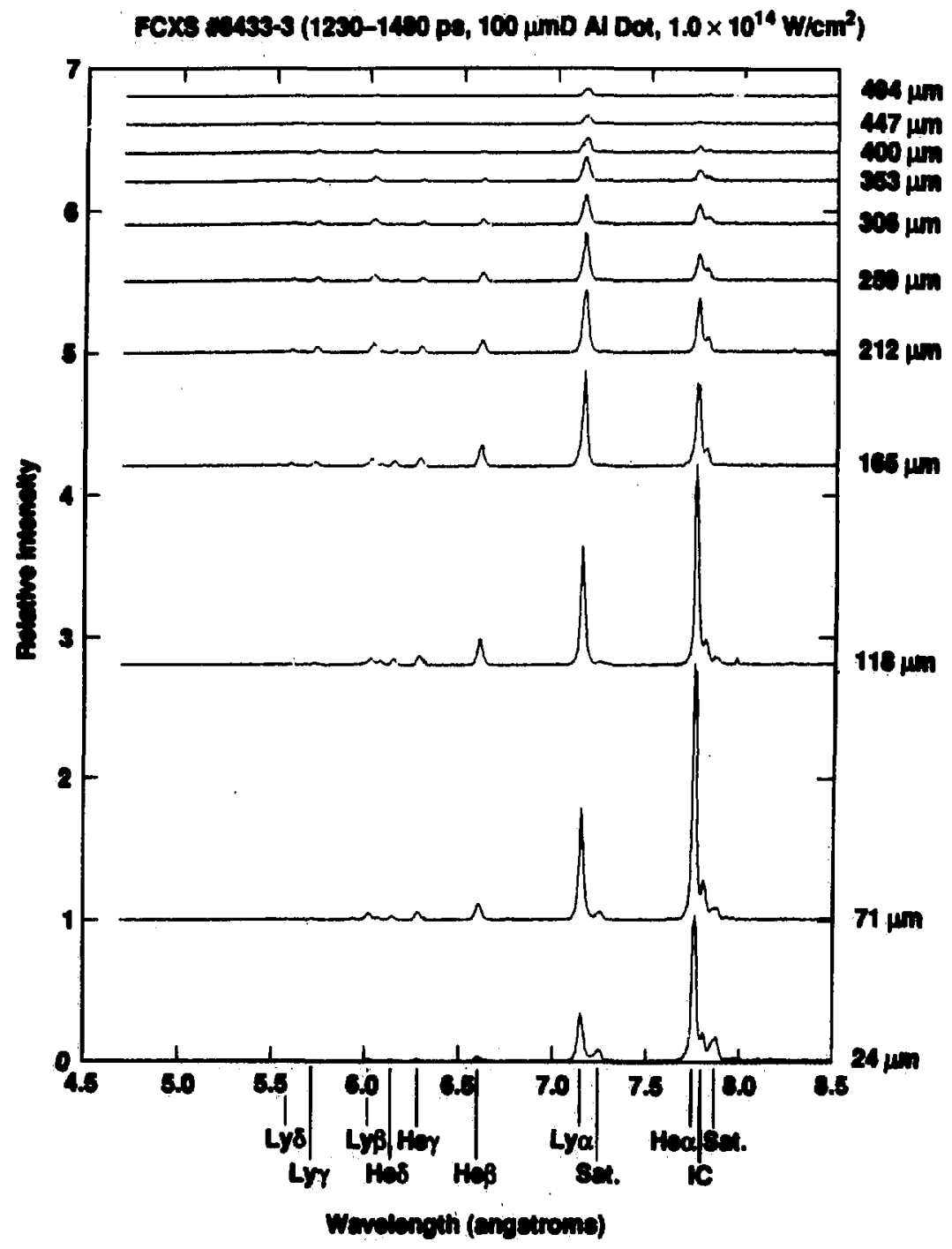




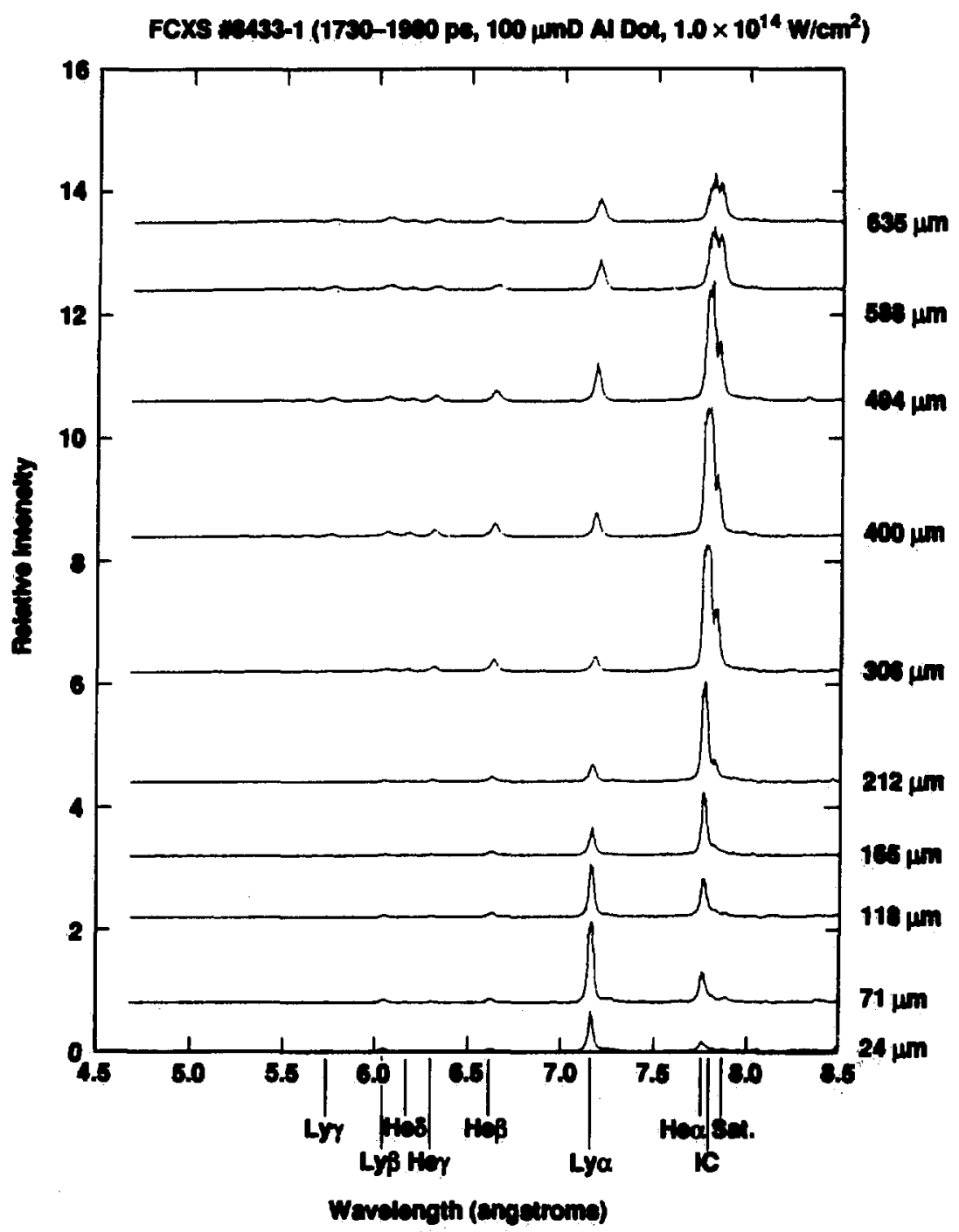

Figure $39 c$ 


\subsection{Measurement of the Electron Temperature Profiles: \\ Temperatures Determined Via the Free-Bound Recombination}

Continuum

The laser-plasma must be characterized in detail to properly investigate and isolate the density sensitivity of various lines. This requires clear and independent measurements of the space and time resolved electron density and temperature profiles. The electron density profiles were measured via independent and simultaneous holographic interferometry measurements. The holographic interferometer was described in Chapter 2. All densities used in this analysis represent values on axis. As noted earlier, the measured density profiles typically varied by less than $10 \%$ across the region (radial) of plasma corresponding to the $\mathrm{Al}$ dot plasma. [See Chapter 2.] The electron velocity distribution can be determined from the shape of the continuum emission spectrum arising from the free-bound recombination continuum of $\mathrm{H}$-like (and also He-like) ions. Since the wave functions for hydrogenic ions are well known, the recombination cross sections for these ions can be calculated accurately from the inverse photoionization process. The energy dependence of the photorecombination cross sections for the hydrogenic ions are well known and these cross sections are relatively insensitive to details of the ionic structure of the recombining species for other ions. [Griem (1964)] Thus, the free-bound recombination continuum can serve as an important electron temperature diagnostic if the plasmas is optically thin to the free-bound recombination emission. This condition is satisfied for the laser-produced plasmas studied in this work. For the simple Al microdot target plasmas, the H-like free-bound recombination continuum lies spectrally isolated from any bound-bound line transitions further insuring that the plasma is optically thin to the continuum radiation. 
In these experiments, the observed electron velocity distributions were well described by a Maxwellian distribution corresponding to a single temperature. This was expected since for the $0.526 \mu \mathrm{m}$ laser light and laser intensities $\left(\leq 10^{14} \mathrm{~W} / \mathrm{cm}^{2}\right)$ used in these experiments, the production of suprathermal electrons should not be a factor. [Note: $v_{\text {osd }} / v_{\text {th }}$ (where $v_{\text {oxc }}$ is the quiver velocity of the oscillating electrons in the field of the laser and $v_{t h}$ is the electron thermal velocity) is generally too small to distort the cold plasma electrons to a non-Gaussian distribution.] [Langdon (1980); Max (1981)]

The free-bound recombination continuum emission intensity can be calculated using the expressions from De Michelis and Mattioli (1981) or by Griem (1964):

$I_{b}(v)=6.4 \times 10^{-40} \cdot \frac{n_{e} n_{z}^{i}-z_{b b} Z^{2}}{\left(k T_{a}\right)^{1 / 2}} \cdot\left[2 \cdot \frac{\left(\frac{z^{2} \cdot \chi_{n}}{k T_{e}}\right)}{n^{3}} \cdot \exp \left(\frac{z^{2} \cdot \chi_{n}}{n^{2} \cdot k T_{e}}\right)\right] \cdot \exp \left(\frac{-\left(\Delta E_{c}^{Z-1}+h v\right)}{k T_{e}}\right)$ in $\left[\mathrm{J} \cdot \mathrm{sr}^{-1} \cdot \sec \right]$, where $\mathrm{n}_{e}$ is the electron density $\left[\mathrm{cm}^{-3}\right], \mathrm{n}_{\mathrm{z}}{ }^{\mathrm{i}}$ is the recombining ion density typically the ground state density $\left[\mathrm{cm}^{-3}\right], x_{n}=13.6 \mathrm{eV}$ where a hydrogenic ionization potential has been used, $\Delta \mathrm{E}_{\mathbf{s}} \mathrm{Z}-1$ is the energy corresponding to the series limit $(n \rightarrow+\infty)$ for the 1s $-n p$ transition, hy is the photon energy above the series limit, and $\left\langle\mathrm{g}_{\mathrm{B}}\right\rangle$ is the average free-bound Gaunt factor, which has been calculated by a number of authors including Karzas and Latter (1961). Typically, $\left\langle\mathrm{g}_{\mathrm{fb}}\right\rangle$ can be set equal to 1 which is a good approximation for the conditions characteristic to these experiments. The key term in the recombination rate is the exponential Boltzman factor which contains the most sensitive electron temperature dependence. The free-bound continuum emission can be fit to the following exponential expression 
with the slope of the $\ln \left[I_{\mathfrak{l b}}(V)\right]$ term inversely proportional to the electron temperature, $\mathbf{T}_{\mathbf{e}}$.

$$
\frac{I_{\mathrm{fb}}\left(V_{n}\right)}{I_{\mathrm{fb}}\left(v_{m}\right)} \propto \exp \left[-\frac{h \cdot\left(v_{m}-v_{n}\right)}{k T_{c}}\right]
$$

This is a very straightforward procedure and gives a simple, (relatively) model independent electron temperature diagnostic. Examples of the space and time resolved Al spectra showing the $\mathrm{H}$-like recombination continuum during (Shot $\left.\# 8433-2,730-980 \mathrm{ps}, 47-94 \mu \mathrm{m}, \mathrm{T}_{\mathrm{e}}(\mathrm{fb}) \approx 305 \mathrm{eV}\right)$ and after the heating laser pulse (Shot $\# 8464-3,1450-1700 \mathrm{ps}, 281-328 \mu \mathrm{m}, \mathrm{T}_{\mathrm{e}}(\mathrm{fb})-135 \mathrm{eV}$ ) are shown in Figures $40 \mathrm{a}$ and $40 \mathrm{~b}$, respectively. These data were recorded using the FCXS instrument from similar laser shots $\left(\approx 1.0 \times 10^{14} \mathrm{~W} / \mathrm{cm}^{2}\right)$ on $100 \mu \mathrm{m}$ diameter $\mathrm{Al}$ dot targets.

The electron temperatures were determined from the slope of the free-bound recombination continuum of $\mathrm{H}$-like ions. The actual recorded continuum emission is composed of both the free-bound recombination continuum and the free-free continuum (bremsstrahlung) from the $\mathrm{Al}$ dot target as well as from the surrounding, heated carbon substrate (free-free continuum). A detailed analysis must be made to properly extract the electron temperature from the slope of the recombination continuum emission as well as a second (most likely cooler) temperature for the substrate plasmia using the slope of the bremsstrahlung continuum. It should be noted that both the free-bound recombination continuum and the bremsstrahlung continuum have the same Boltzman factor temperature dependence.

This was not a problem for these experiments. The Al free-bound continuum is clearly distinguishable in the measured Al K-shell spectra. The H-like free-bound recombination continuum begins at approximately the $(n \rightarrow \infty)$ series limit for the 
exponentially with increasing photon energy. This free-bound continuum signature is clearly an $\mathrm{Al}$ feature and is much larger $(210 \mathrm{X})$ than the only slightly cooler free-free continuum, originating primarily from the surrounding carbon substrate plasma. This is shown in Figure 40a, during the heating laser pulse, and represents the FCXS space/time frame in which the free-free continuum emission is the brightest in intensity and contributes the most to the overall recorded $x$-ray emission spectrum. For this shot, the temperature of the spatially averaged carbon plasma was approximutely 20\% cooler than the Al dot plasma. This cooler free-free continuum contribution incresses the $\mathrm{Al}$ free-bound continuum slope by less than 5\%. Thus, the uncorrected Al free-bound temperature is only slightly ( $=5 \%$ maximum) lower than its corrected value and is well within the estimated $\pm 15-20 \%$ margin of error for the reported free-bound continuum temperntures. After the laser pulse, the free-free emission diminishes considerably and contributes only slightly to the overall noise leval as seen in Figure $40 \mathrm{~b}$. It should also be noted that the free-bound continuum signal falls off fairly linearly (exponentially) which is characteristic of a single electron temperature value (for a Maxwellian velocity distribution). For most cases, the slope of the free-bound countinuum could be readily determined to an accuracy of $\pm 10 \%$ or better using a least-squares fit. Therefore, any temperature variation both radially and within the FCXS spatially resolved (axial) increment is expected to be small. Thus, for all space and time frames, if the Al free-bound continuum is measured, the temperature can be readily determined from the slope of the free-bound recombination continuum.

The space and time resolved electron temperature profiles for an Al microdot plasma heated at $1.0 \times 1014 \mathrm{~W} / \mathrm{cm}^{2}$ are shown in Figure 41 (solid curves). These temperature profiles represent four selected time intervals: (top) $730-980$ ps [Shot \#8433]; (upper middle) 950-1200 ps [Shot \#8464]; (lower middle) 1450-1700 ps [Shot \#8464]; and (bottom) $1950-2200$ ps [Shot \#8464]. This is the first 
Figure 40. Typical digitized Al K-shell x-ray spectra recorded with the FCXS clearly shows the H-like free-bound recombination continuum which is used to determine the free-electron velocity distribution for a) Shot $\# 8433(730-980$ ps, $47-94 \mu \mathrm{m})$ and b) Shot $\# 8464(1450-1700$ ps, 328-375 $\mu \mathrm{m})$. For a Maxwellian velocity distribution, the slope of the free-bound recombination continuum (logarithm of the intensity) falls off with energy and is inversely proportional to the electron temperature. The free-bound continuum for these data have been (exponentially) fitted and show temperatures of a) $305 \mathrm{eV}$ and b) $135 \mathrm{eV}$. 


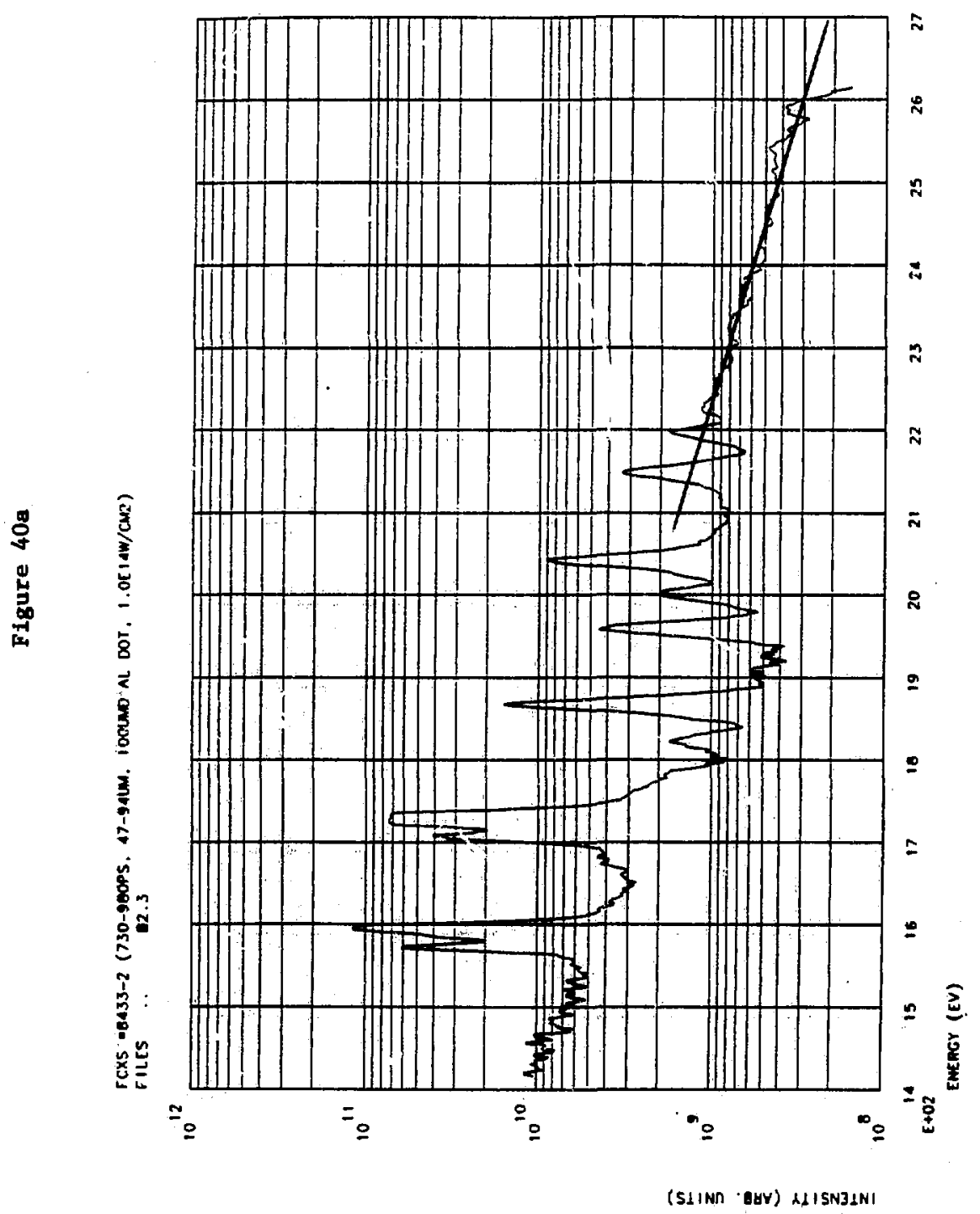




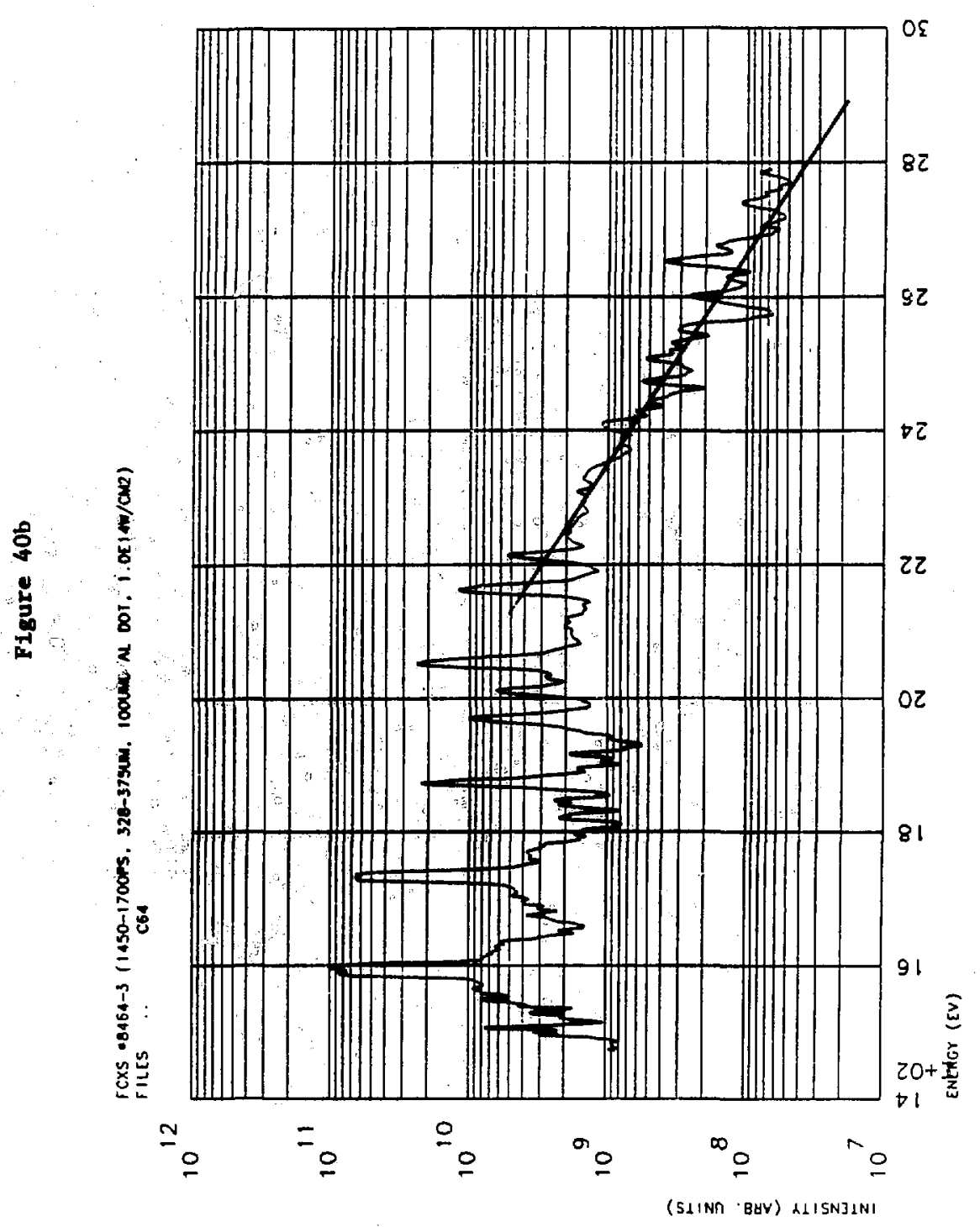


Figure 41. Time resolved temperature profiles determined from the $\mathrm{H}$-like $\mathrm{Al}$ free-bound recombination continuum (solid lines) and from a 2D "LASNEX" simulation (dashed lines) are presented for (top) 730-980 ps, (upper middle) 950-1200 ps, (lower middle) 1450-1700 ps, and (bottom) 1950-2100 ps after the start of the $1.0 \mathrm{~ns}$ laser heating pulse. 

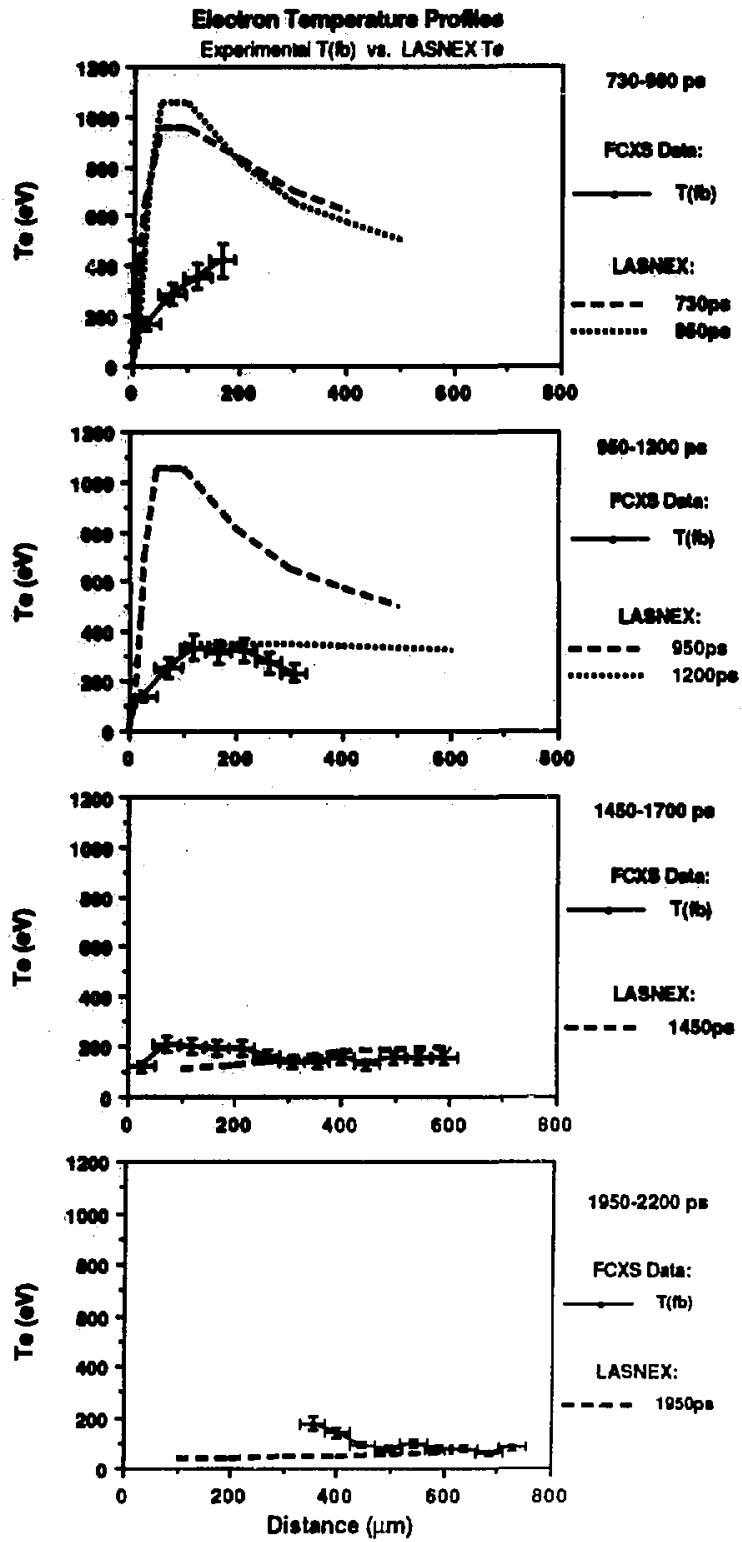

Figure 41 
measurement of the space and time resolved electron temperature profile in the corona of a laser-produce plasma. In combination with the independently and simultaneously measured interferometric density profiles, these new data provide a detailed, fairly model-independent characterization of the plasma. This allows us to clearly study the density and temperature dependences of spectral line intensities as predicted by atomic theory and to begin to examine laser absorption and heat transport phenomena in the laser-plasma corona.

\subsubsection{Comparison to 2D Hydrodynamic Simulations}

The electron temperature and density profiles have also been recently simulated using a two dimensional "LASNEX" simulation by C. Cerjan (1988) and are presented by dashed and dotted lines in Figures 41 and 42, respectively, and reported by Young et al (1988). The "LASNEX" model is a large computer code which calculates the hydrodynamics of the specified laser-matter interaction, accounting for the absorption of the heating laser light, heat transport, plasma generation and transport, thermal conduction in the plasma, etc. The "LASNEX" code has been fully described elsewhere. [Zimmerman (1975); Cerjan (1988)] For these calculations, a two dimensional cylindrically symmetric simulation was performed. The electron thermal conduction was assigned a classical flux limiting value of 0.4 . The atomic kinetics were described by a hydrogenic approximation rather than the less realistic average-atom approximation. Radiation transport was treated in the diffusion approximation, except in the x-ray regions of interest where an escape probability method was used. The simulation also assumed inverse bremsstrahlung laser light absorption and classical $T^{5 / 2}$ thermal conduction. These are typical "LASNEX" parameters used to simulate laser-plasma interactions under these laser conditions. [For example, see Rosen (1987); Max (1980); Burkhalter et 
Figure 42. Density profiles (center line) of an Al microdot plasma determined from holographic interferometry (solid lines) and from a 2D "LASNEX" simulation (dashed lines) are presented for (top) 657 ps, (upper middle) 857 ps, (lower middle) $1257 \mathrm{ps}$, and (bottom) $1657 \mathrm{ps}$ after the start of the $1.0 \mathrm{~ns}$ laser heating pulse. 

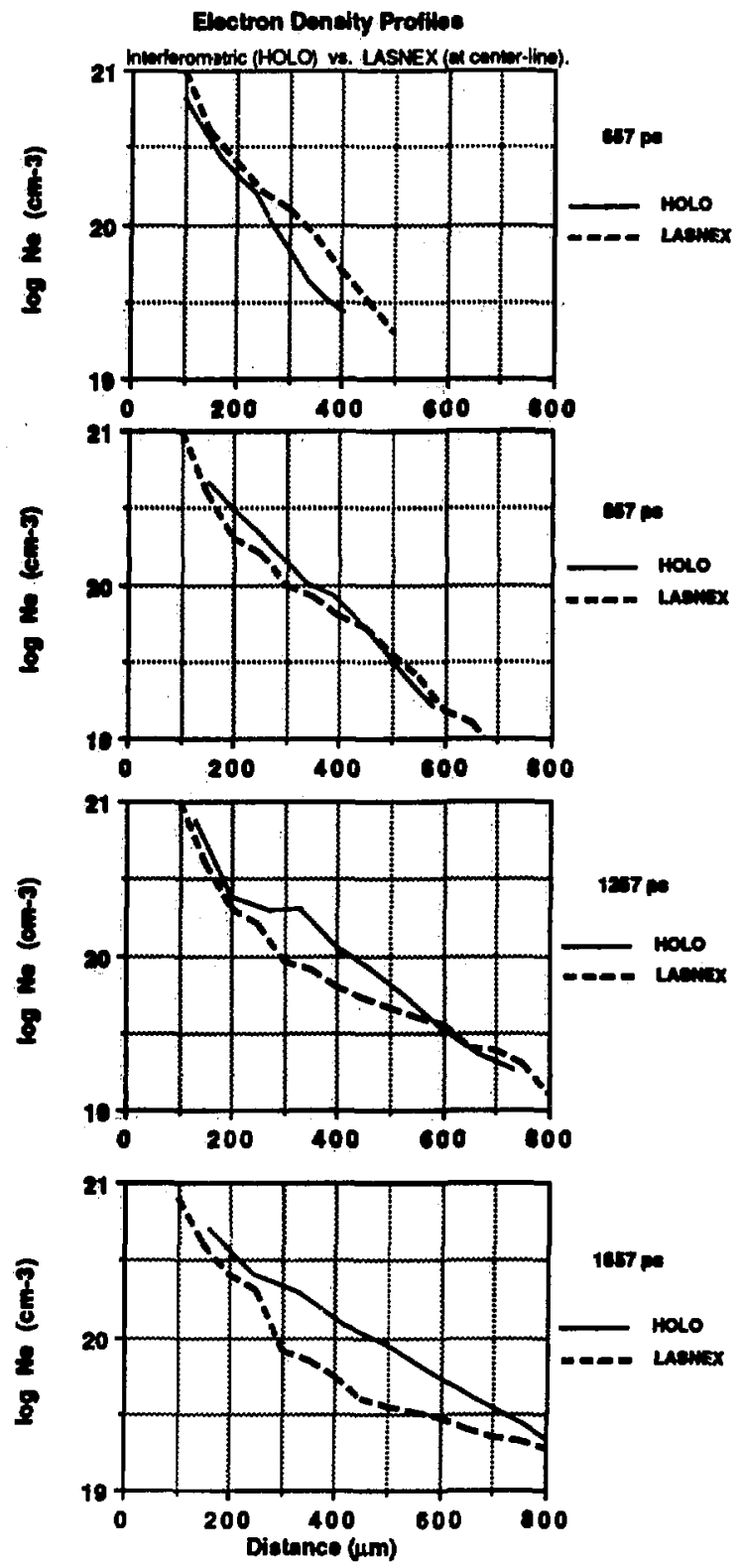

Figure 42 
al. (1982).] Both the electron density and temperature (radial) profiles were predicted to be relatively flat ( $\pm 10 \%$ variation) across the $\mathrm{Al}$ dot plasma as shown eariier in Figure 2. The electron temperatures representing the outer $\mathrm{Al}$ zones have been used for the present analysis.

The elestron density profiles predicted by the "LASNEX" simulation are generally in good agreement with experiment. Measurements of the electron density profiles using the multiple frame holographic interferometry and the "LASNEX" code predictions representing 657, 857, 1257, and 1657 ps are shown in Figure 42. [Shots $\# 8460$ and $\# 8464$ at $1.0 \times 10^{14} \mathrm{~W} / \mathrm{cm}^{2}$ ] The electron densities shown correspond to the density at the center (on axis) of the microdot plasma. The "LASNEX" predicted profiles are in very good agreement with experiment while the heating laser is on but are generally lower after the laser is off. An overly rapid expansion of the plasma in the transverse (radial) direction could account for a more rapid radiative cooling rate and therefore greater decrease in the electron density in the "LASNEX" simulation, especially at large distances from the target surface and at late times. This phenomena is not understood and will be the focus of future work.

The temperature profile observed while the laser is on $(730-980 \mathrm{ps})$ is significantly different than the "LASNEX" calculation. [Figure 4l(top).] The measured temperatures are a factor of three lower than predicted near the target surface and continually rise for at least $200 \mu \mathrm{m}$ out into the plasma corona while the predicted temperatures peak at approximately $50 \mu \mathrm{m}$ and monotonically decrease from $100 \mu \mathrm{m}$ outward in the simulation. Even the (CRE) line intensity ratio deduced temperatures (to be discussed in the following section) show the same qualitative deviation from the simulation. Systematic experimental uncertainties such as differences between the experimental and modeled heating laser intensity profiles or a small misalignment of the microdot target within the laser focal spot are not likely to fully account for the 
large observed differences. These anomalously low measured electron temperatures are similar to the coronal temperature of $-400 \mathrm{eV}$ measured by Drake et al. (1988) using Raman scattering. Their measurements were for similar irradiance conditions

$$
\left(1.0 \times 10^{14} \mathrm{~W} / \mathrm{cm}^{2} ; \lambda=0.35 \mu \mathrm{m}\right) \text { during the heating laser pulse at a density of }
$$
approximately $1.0 \times 10^{21} \mathrm{~cm}^{-3}$ (approximately the density of the Al plasma at 100 $\mu \mathrm{m}$ from the target suface in my experiments). In another related experiment by

Bernard et al. (1987) using high intensity $\mathrm{CO}_{2}$ laser light $(\lambda=10.6 \mu \mathrm{m})$, experimental temperatures measured using Raman scattering were found to be approximately a factor of 2 lower than 1D "LASNEX" simulations. For that experiment, the differences were due to a distortion of the classical inverse bremsstrahlung absorption coefficient. For the present experiments, this is not expected to be a significant effect because of the short laser wavelength and moderate laser intensities used in the experiments. Instead, this new data may indicate absorption or transport mechanisms are not fully accounted for in the simulation. Further experiments of this type should prove useful in understanding the discrepancy.

For the second time frame (950-1200 ps), it is difficult to compare the experimental and predicted temperature profiles because the FCXS time frame straddles the end of the heating laser pulse. [Figure 41(upper middle).] I have plotted the temperature profiles at 950 ps and 1200 ps calculated by the "LASNEX" code with the FCXS free-bound temperature in Figure 41(upper middle). The observed temperatures are much lower that those predicted at $950 \mathrm{ps}$. The observed temperatures are in better agreement with the "LASNEX" simulations at 1200 ps although with much more (spatial) structure than predicted. The closer agreement with the later time is expected since the free-bound recombination cross section is larger at lower temperatures and therefore the measured free-bound temperature will 
difficult to measure due to its low emissivity and poor signal-to-noise level.

\subsection{Temperature Dependence of $\mathrm{K}$-shell Line Intensities}

Often, the complexity and energy range of the line emission spectra from complex laser targets prevent the clear measurement of the energy distribution of the free-bound continuum. In these cases, the electron temperature can be derived from emission line intensity ratios based on detailed atomic model calculations. The temperature dependence of these line ratios is due to the functional form of the temperature dependence of different atomic processes feeding the excited levels. A detailed discussion of the theory of the temperature dependence of various $\mathrm{K}$-shell lines will not be presented here. Instead, I refer the reader to some excellent reviews by DeMicheles and Mattioli (1981), Griem (1964), Key and Hutcheon (1980), Wilson (1962), and McWhirter (1965). For simplicity, only a brief analysis of the new spectroscopic data using the standard, collisional radiative equilibrium (CRE) interpretations will be presented here. This discussion is meant only to illustrate the traditional interpretation of the temperature dependence of various line intensity ratios. The highly transient nature of these laser-produced plasmas indicates that a dynamic modeling scheme is required to properly model the temperature dependence of the line intensity ratios or conversely, to use measured line intensity ratios to deduce plasma parameters (such as the electron temperature, density, and charge state distribution) characteristic of the plasma.

\subsubsection{K-shell Resonance Line Ratios}

A commonly used method for estimating the electron temperature is to measure the line ratio between the resonance lines of two adjacent charge states and infer the 
temperature from equilibrium ionization models. For example, the He-like $1 s^{2}\left({ }^{1} S_{0}\right)$ - 1s $2 \mathrm{p}\left({ }^{1} \mathrm{P}_{1}\right)$ "He ${ }_{\alpha}$ " to the $\mathrm{H}$-like $1 \mathrm{~s}-2 \mathrm{p}$ "Ly ${ }_{\alpha}$ " resonance line ratio is commonly used. Often, these $(n=1)$ to $(n=2)$ resonance lines are optically thick and higher lying lines such as the He-like $1 s^{2}\left({ }^{1} S_{0}\right)-1 s 3 p\left({ }^{1} P_{1}\right)$ "He$\beta$ " to the H-like $1 s-3 p$ "Ly" $y_{\beta}$ resonance lines are used instead to reduce uncertainties in the measure line intensities due to opacity effects. For this example, I will assume that the excited levels are populated only by excitations from their relative ground states and not from other processes such as recombination and ionization. Ideally, the energy levels representing the larger principal quantum numbers ( $\mathrm{n} \geq 7$ ) and corresponding to the higher lying resonance transitions will be in local thermodynamic equilibrium (LTE ) with the higher energy levels including the continuum of free electrons. At high densities $\left(\mathrm{n}_{\mathrm{e}}>10^{22} \mathrm{~cm}^{-3}\right)$, the high $\mathrm{n}$ resonance line ratio approaches the LTE limit. The LTE resonance line intensity will be proportional to the population density of the next ionization stage $\mathrm{Z}+1$ and therefore the He-like to $\mathrm{H}$-like 1 s-np resonance lines will have the following ratio:

$$
\frac{I_{H_{e}}(n, 1)}{I_{H}(n, 1)}=\frac{A_{H e}(1, n) \cdot g_{z} \cdot g_{H e}\left(n^{\prime}\right) \cdot n_{H}(1)}{A_{H}(1, n) \cdot g_{H}(n) \cdot g_{H}(1) \cdot n_{B}(1)} \cdot \exp \left[\frac{\chi_{H_{e}}\left(n^{\prime}, \infty\right)-\chi_{H}(n, \infty)}{k T_{e}}\right]
$$

where $\mathrm{B}, \mathrm{H}$, and $\mathrm{He}$ represent the bare, $\mathrm{H}$-like, and He-like ions, respectively. Therefore, if the relative abundance of the various charge states is known, a value for the electron temperature may be derived from the above expression. This charge state distribution can be readily obtained by assuming an equilibrium ionization distribution such as CRE. The "He $\beta / \mathrm{Ly}_{\beta}$ " line intensity ratio has been plotted in Figure 21d as a function of electron temperature for a range of densities. These plots have been generated using the "RATION" (CRE) model and are shown for both the 
optically thin (left) and thick [ $50 \mu \mathrm{m}$ plasma size] (right) cases.

The temperature sensitivity of this line ratio depends entirely on the charge state distribution in the plasma. Therefore, the validity of this line ratio as a temperature diagnostic is based on knowing the particular charge state fraction. The CRE expression for the resonance line ratio is dependent on the basic assumption of having an equilibrium charge state distribution. If the plasma is non-stationary, the temperature dependence of this resonance line ratio will fail. For typical laser-produced plasmas, the charge state distribution is very far from equilibrium. Ionization times are typically of order of several hundred $100 \mathrm{ps}$ and therefore a time dependent analysis is necessary. This is in contrast to the picosecond collisional and fast radiative decay rates $\left(10^{13} \sec ^{-1}\right)$ time acales which govern the level population kinetics of the density sensitive transitions studied in this work. In the non-stationary case (if the plasma is recumbining or ionizing), the resonance line ratio alone cannot be used to correctly measure the electron temperature. However in principle, the ratio might be fit to a time dependent calculation including transient ionization effects. The best procedure is to solve for the ionization distribution by. analyzing a family' . spectral lines and simultaneously determining the relative charge state distribution, the electron density, and temperature.

\subsubsection{Dielectronic Satellite to Resonance Line Ration}

In the $x$-ray spectra of laser-produced plasmas, the resonance lines of highly ionized $\mathrm{H}$-like and He-like ions are often accompanied by satellite lines from doubly excited states of ions from the next lower charge state (He-like or Li-like, respectively). These satellite lines originate from doubly excited levels which are populated by i) dielectronic recombination and ii) inner-shell excitation of the He-like (Li-like) ion and are interesting since their intensities may be interpreted to determine 
the electron temperature or density of the plasma. For example, the H-like $(1 \mathrm{~s}-2 \mathrm{p})$ "Ly $y_{\alpha}$ " resonance line to the He-like $\left[1 s 2 p\left({ }^{1} P_{1}\right)-2 p^{2}\left({ }^{1} D_{2}\right)\right]$ dielectronic satellite line ratio can be used as a temperature diagnostic. For the present work, I will consider only the former process of dielectronic recombination. Inner-shell excitation is an important mechanism for Li-like satellites but do not contribute significantly to the He-like satellites lines used in this work.

Dielectronic recombination can be considered as a multi-step process:

1) Dielectronic capture occurs when a colliding electron (below the threshold energy) excites the ion to a reșonance transition and gets itself captured into an outer orbital. If at this point autoionization cocurs, the ion relaxes to its original state and no recombination occurs.

2) Alternatively, the doubly excited state can stabilize by the radiative decay of the inner excited electron. A resonance photon will be produced by the ion stifted slightly to longer wavelength due to the influence of the additional outer (screening) electron.

3) Lastly, the now singly excited ion relaxes by cascades to the ground state.

The two main effects on the observed $x$-ray emission are i) the introduction of weak "satellite" lines occurring on the long wavelength side of the associated resonance lines, and ii) the enhancement of the resonance lines due to unresolved dielectronic satellite lines corresponding to doubly excited states with the outer electron having high principal quantum numbers of $n \geq 4$. For the latter case, dielectronic satellites are typically indistinguishable in wavelength from the equivalent resonance line and contribute to the measured resonance line intensity. A third, more subtle effect, is that dielectronic recombination increases the total recombination rate into that particular charge state which impacts the overall charge state distribution. 
The He-like dielectronic satellite line comes from the resonant electron capture of the 1s H-like ground state to a doubly excited He-like states [for example: $2 \mathrm{p}^{2}\left({ }^{1} \mathrm{D}_{2}\right)$ ]. The capture rate coefficient typically peaks at a lower electron temperature than the associated resonance line primarily due to the difference in transition energies. [For example, the H-like [1s $\left.\left({ }^{1} S_{1 / 2}\right)-2 p\left({ }^{1} P_{3 / 2}\right)\right]$ "Ly $x^{\prime}$ resonance line.] The resul'. is the dielectronic satellite line intensity tends to fall off relative to its adjacent or "parent" resonanse line intensity as a function of the electron temperature and independent from the electron densit. Therefore, the resonance line to dielectronic satellite line ratio can provide a clean electron temperature diagnostic.

The theory for the temperature dependence of the dielectronic satellite to resonance line intensity ratio hes been developed by Gabriel (1972) and Dubau et al. (1980; 1981) and is described ir most review articles on spectroscopic methods for high temperature plasmas. I briefly outline the procedure of Dubau. The $T^{\circ}$ tive intensity ratio between the satellite and resonance line ratio can be written as:

$$
\frac{I_{S}}{I_{R}}=F_{1}\left(T_{e}\right) \cdot F_{2}(S)
$$

where the temperature dependence of the ratio has been isolated in the term $F_{1}\left(T_{e}\right)$. $\mathrm{F}_{2}(\mathrm{~S})$ is a term containing the relative autoionizution and moliative decay rates characteristic of the particular satellite line.

$$
\begin{aligned}
& F_{1}\left(T_{e}\right)=3.038 \times 10^{-13} \cdot \frac{1}{\langle f\rangle} \cdot \frac{E_{0}}{T_{e}} \cdot \exp \left[\frac{\left(E_{0}-E_{S}\right)}{k T_{e}}\right], \\
& F_{2}(S)=\frac{g_{S} \cdot A_{a} \cdot A_{t}}{\left(A_{a}+\sum A_{r}\right)}
\end{aligned}
$$

where $A_{\mathbf{a}}$ and $A_{r}$ are the autoionization and radiative decay rates in $\sec ^{-1}$, 
respectively, $g_{s}$ is the statistical weight, $\Sigma A_{r}$ is the sum over all possible final states, $T_{e}$ is in ${ }^{\circ} K, E_{0}$, and $E_{s}$ are the energies of the resonance and satellite levels relative to the $H$-like ground state in $\mathrm{eV}$, and $\langle f\rangle$ is the effective oscillator strength for collisional excitation of the resonance line. It should be noted that the $Z$ dependence lies primarily in the radiative transition probability, $A_{n}$ which behaves like $Z^{4}$. Therefore, dielectronic satellites are more apparent for high $\mathrm{Z}$ plasmas. The factor $\left(E_{o}-E_{2}\right) k T_{e}$ is usually smaller than unity and contributes only as a slowly varying function of $T_{a}$. The main temperature dependences arises from the $T_{e}{ }^{-1}$ term in $F_{1}\left(T_{e}\right)$. Therefore, thu dielectronic satellite line intensity is expected to fall off in intensity relative to the resonance line as $T_{e}^{-1}$.

An analytic expression for $<f>$ derived by Vainstein (1975) is:

$$
\langle f\rangle=\left(\frac{E_{2}}{E_{1}}\right)^{3 / 2} \cdot \frac{\beta}{6.27} \cdot\left[\frac{E_{0} k T_{e}+\delta_{s_{1} s_{2}}}{E_{0} / T_{e}+\chi}\right],
$$

where $E_{2} / E_{1}$ is the energy ratio of the resonance line states, $\delta_{s_{1} s_{2}}$ is the spin factor, and $\beta$ and $\chi$ are fitting parameters which depend on the type and ionization state of the transition considered.

Vainstein (1975) finds empirical values for the above parameters which correspond to the ground state excitation of the H-like resonance line are: $E_{2} / E_{1}=0.25, \delta_{\mathrm{S} 1 S 2}=$ $1, \beta=17$, and $\chi=0.23$. The temperature dependence of this line intensity ratio based on "RATION" (CRE) code is shown in Figure 2le for both the optically thin (left) and thick [ $50 \mu \mathrm{m}$ size] (right) plasmas. 


\subsection{Experimental Line Intensity Ratio Temperatures}

Electron temperatures deduced from standard line intensity ratio techniques have been compared to the model independent free-bound recombination continuum temperatures. I have considered the line ratios between the He-like $\left[1 \mathrm{~s}^{2}\left(1 \mathrm{~S}_{0}\right)-\right.$ 1s3p $\left.\left({ }^{1} P_{1}\right)\right]$ and $\mathrm{H}$-like $1 \mathrm{~s}-3 \mathrm{p}$ resconance lines "Hep $/ \mathrm{Ly}_{\beta}$ ", and between the H-like $(1 s-2 p)$ and $(1 s-3 p)$ resonance lines and He-like $\left[1 s 2 p\left({ }^{1} P_{1}\right)-2 p^{2}\left({ }^{1} D_{2}\right)\right]$ dielectronic

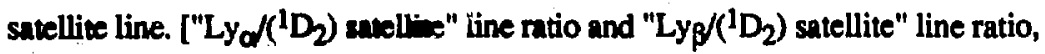
respectively.] Although the latter "Ly $/\left({ }^{1} D_{2}\right)$ satellite" line ratio is somewhat unconventional to use, it still effectively maintains the electron temperature dependence as the more common "Ly $\alpha /\left({ }^{1} D_{2}\right)$ satellite" line ratio, yet the "Ly" resonance line is optically thinner and not subject to overexposure (saturation) problems which affected the "Ly ${ }_{\alpha}$ " line in these experiments. The "RATION" (CRE) "Lyp/('D $\left.{ }^{2}\right)$ satellite" line raio yersus temperature calculation is shown in Figure $21 \mathrm{f}$ for boh en optically thin (left) and thick [ $50 \mu \mathrm{m}$ plasma size] (right) plasma. Because the "Ly $y_{\beta}$ " line appears somewhat spectrally away from the " $\left({ }^{1} D_{2}\right)$ sabellite" line, there may be some question as to their measured (relative) intensities due to the possible uncertainties in the instrumental response. This should not be a significant problem but it needs to be addressed in future work. The line ratio temperatures were desived by comparing the measured line intensity ratios with those predicted by the "RAT1ON" (CRE) code [R. Lee et al. (1985)]. These temperature profiles are compered with the true temperature profiles derived from the free-bound continuum and are shown in Figure 43. The free-bound continuum temperatures are derived from Shots $\# 8433$ and $\# 8464$ while the line intensities were measured using 
Figure 43. A comparison of electron temperature profiles measured from the $\mathrm{H}$-like free-bound recombination continuum and determined from various "RATION" (CRE) analyzed line intensity ratios, including the "Ly $\beta /\left({ }^{1} D_{2}\right)$ satellite" line and "He $/$ Ly $y_{\beta}$ " ratios for (top) 450-700 ps (Shot $\# 8460$ ), (middle) 730-980 ps (Shots \#8433 \& \#8459), and (bottom) 950-1200 ps, (top; second page) $1450-1700$ ps, and (bottom; second page) 1950-2200 ps (Shots $\$ 8464$ \& \#8465). 

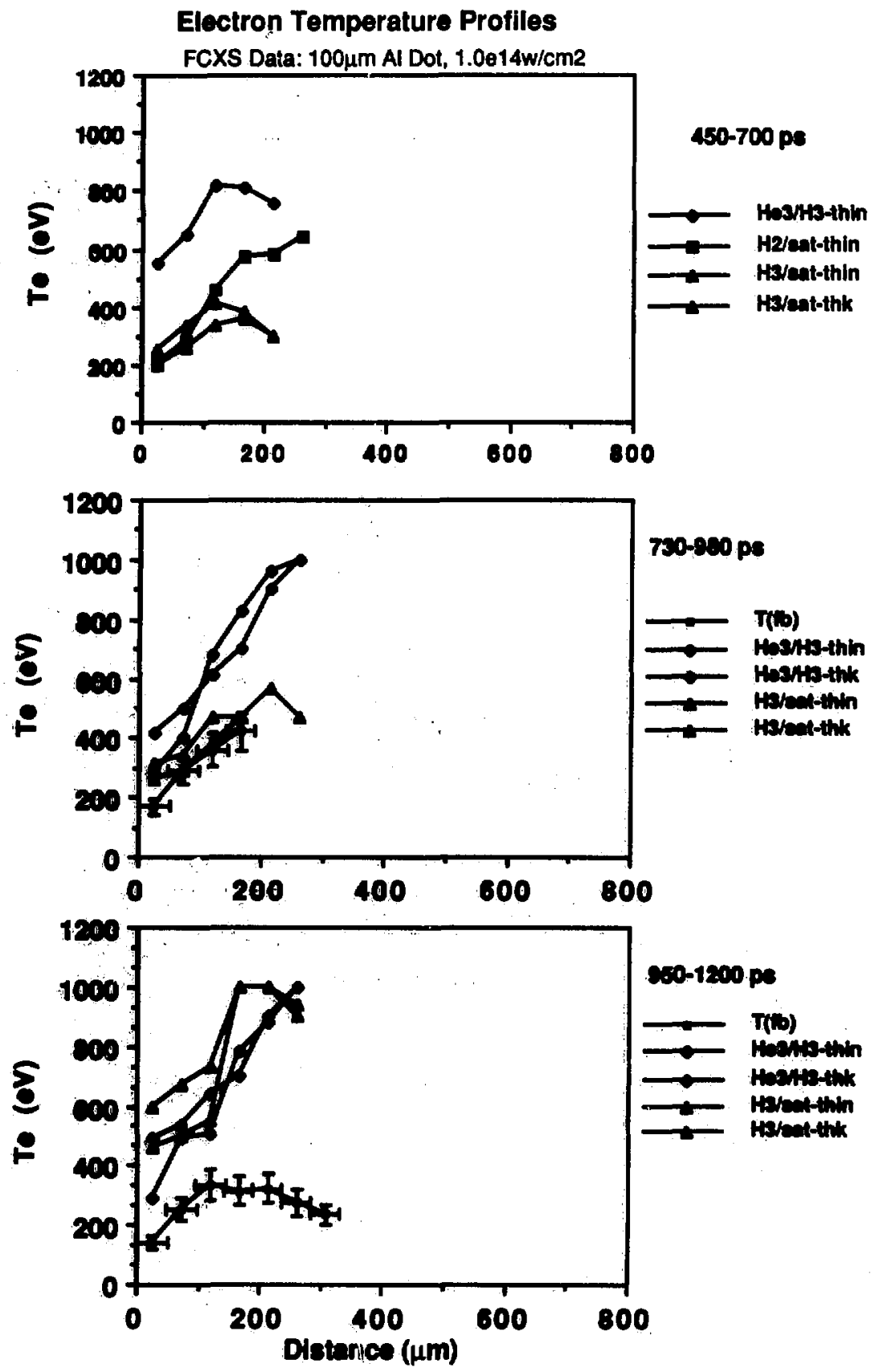


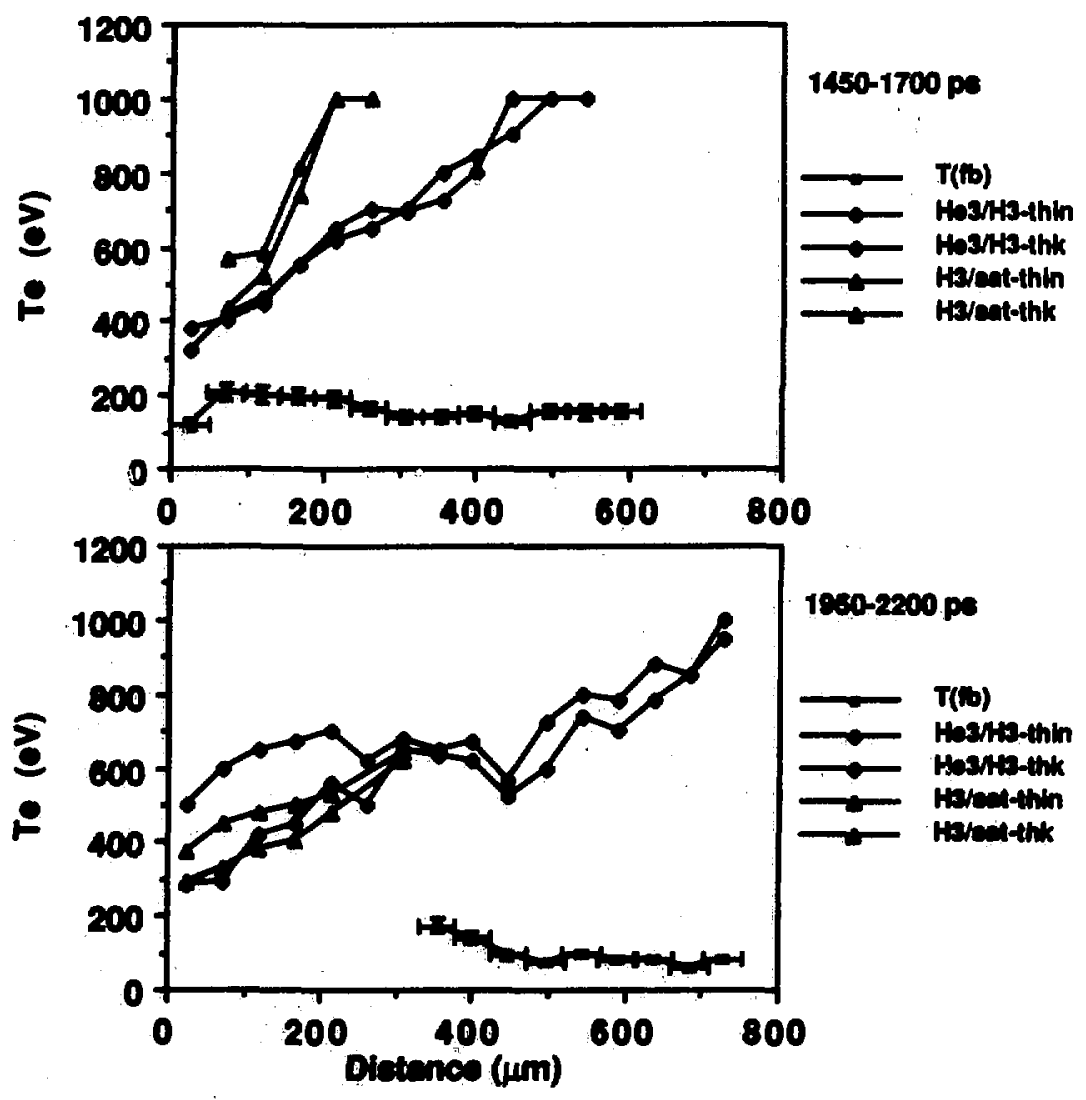

Figure 43b 
Shots $\# 8433, \# 8459, \# 8460$, and $\# 8465$. Different target shots were used because the resonance lines were typically overexposed for the free-bound continuum frames as described earlier. Again, the FCXS was used to measure this data and these shots hed very similar laser conditions $\left(1.0 \pm 0.1 \times 10^{14} \mathrm{~W} / \mathrm{cm}^{2}\right)$ on $100 \mu \mathrm{mD} \mathrm{Al}$ dot targets.

Optical trapping effects were included in the CRE model using a simple escape factor correction. [Cauble (1987)] The H-like 1s-2p resonance lines are predicted to be optically thin for low temperatures $(<500 \mathrm{eV})$ and densities below $10^{21} \mathrm{~cm}^{-3}$ typical for the laser-plasmas studied in this work. This is due to the CRE code computing a plasma (line) opacity based on a predicted equilibrium charge state distribution. For these low temperatures and densities, the "RATION" model predicts only a very small amount of $\mathrm{H}$-like ions and therefore all $\mathrm{H}$-like lines are computed to be optically thin. Therefore, the "Ly/ $/\left({ }^{1} D_{2}\right)$ satellite" line ratio is computed to be optically thin and the predicted temperatures are generally independent of plasma opacity. The "Hep/Ly $\beta$ " line ratio deduced temperatures are also not affected. This is probably incorrect as there is an appreciable amount of H-like ions in the laser-plasma (far more than predicted by the CRE model) and the "Ly ${ }_{\alpha}$ "line is likely to be optically thick. Therefore, I have used the "Ly $/\left({ }^{1} D_{2}\right)$ sucllive" line ratio instead of the more common " $\mathrm{Ly}_{\alpha} /\left({ }^{1} \mathrm{D}_{2}\right)$ satellite" line ratio to reduce the uncertainty due to the opacity effects. I expect that true opacity corrections will mainly affect the resonance line intensities (and probably not the satellite line) and the temperatures derived using the optically thick resonance to satellite line ratio should be higher relative to an optically thin prediction. The differences between the resonance to satellite line ratio temperatures and the free-bound temperatures should increase if the opacity effects are properly accounted for. The densities used in the interpretation of the measured line ratios were 
determined from interferometry measurements.

While the laser is on [See Figure 43(top)], the electron temperature profile derived using the CRE "Ly $\beta /\left({ }^{1} D_{2}\right)$ satellite" line ratio agrees with the temperatures measured from the recombination continuum for the plasma region between the target surface and approximately $200 \mu \mathrm{m}$ out into the plasma plume. Beyond this region, the satellite line ratio temperature is somewhat higher than the free-bound temperature. This may indicate a transition zone between a near-equilibrium (CRE) plasma (near the surface during the heating laser pulse) and a predominantly recombining plasma further out in the plasma plume where the plasma is clearly far from equilibrium and the CRE satellite line ratio temperatures are much higher than the true (free-bound) temperatures. [Note: this is approximately the same plasma region in which the recombination density dependence of the " $\alpha$ " line ratio fails for the $10 \% \mathrm{Al}$ dot. (See Section 5.5.1.)] The failure of the equilibrium plasma model indicates that a non-stationary atomic model analysis is needed to resolved these differences.

It should be noted that the He-like $\left[1 s^{2}\left({ }^{1} S_{0}\right)-1 s 2 p\left({ }^{1} P_{1}\right)\right] ~ " H e_{\alpha}$ " resonance line to Li-like [1s22p(2P) - 1s2 $\left.p^{2}(2 \mathrm{D})\right]$ "jkl" dielectronic satellite (blended) line ratio was also examined to determine the electron temperature although this data is not presented here. In general, the "He $/ j k l$ sutellite" line ratio predicted similar ( $(20 \%)$ electron temperature values compared to the temperature determined from the "Ly $\beta /\left({ }^{1} D_{2}\right)$ satellite" line ratio. This data was more difficult to interpret due to the low emissivity of the Li-like satellite lines and due to unresolved satellite lines. Additionally, the " $\mathrm{He}_{\alpha}$ " line was predicted to be significantly (optically) thick and its interpretation more likely to be model dependent and therefore suspect. Although the resonance to satellite line ratio has been successfully used to characterize tokamak and solar corona plasmas, the present data represents the first clear evidence of 
agreement between dielectronic satellite to resonance line ratio deduced temperatures with theory for a laser-produced plasma.

The CRE temperatures determined using the " $\mathrm{He}_{\beta} / \mathrm{Ly}_{\beta}$ " resonance line ratio are always higher than the true (free-bound) temperatures. This indicates that the plasma is slightly overionized with respect to its temperature and density, leading to higher apparent temperatures deduced from the "He$/ L y_{\beta}$ " resonance line ratio. This behavior is consistent with previous measurements, and is due to the large velocities of the Al icus through the electron density gradient present in these plasmas. During the time frame (730-980 ps) when the heating laser pulse is on, the dielectronic satellite line ratios are in much closer agreement with theory despite this apparent overionization. This is not surprising since this ratio is less sensitive to the ionization distribution because both lines originate primarily from the $\mathrm{H}$-like ground state (assuming a non-recombining plasma).

After the laser pulse, the plasma clearly is recombining and the resonance lines are populated significantly by recombination from higher charge states. Both the "Hep/Ly $y_{\beta}$ " resonance line and the satellite line ratio deduced temperatures are far higher than the true temperatures. [See Figures 43(upper middle), 43(lower middle), and 43(bottom).] This becomes more and more apparent later in time as the plasma cools off and farther out into the plasma plume as recombination becomes the dominant atcimic process affecting the population kinetics of the excited levels. In order to conpensate for the lack of ionization equilibrium in the plasma, the ionization distribution should be measured experimentally and independently based on the recorded spectral emission. This might be done by detailed time dependent modeling of the stripping of ions near the critical surface such as suggested by Kauffman et al.(1987) and Cerjan (1988). This assumes that the ionization processes are modeled correctly in the hydrodynamics code. An alternative method 
would be to derived the ionization distribution directly from the spectrum without recourse to hydrodynamic simulations by considering the values of both resonance line and dielectronic satellite ratios simultaneously and systematically solving for the relative ion distribution and plasma temperature and densities as suggested by Boiko (1983) and Goldstein (1986). A preliminary version of this quasi-steady state analysis and model is applied to examine the density dependence of various He-like lines in the remainder of this chapter.

\subsection{Density Sensitive He-like Lines: Results and Discussion}

The electron density profiles were measured using holographic interferometry. The center-line (axial) density profiles derived from the Abel inversion are shown in Figure 19 for a sequence of times and present a clear picture of the space-time history of the electron density of a simple laser-produced plasma. Electron density profiles were also determined spectroscopically using the FCXS data. Both density measurements are simultaneously space and time resolved and thus provide a direct test of the density dependence of specific spectral lines. The standand line intensity ratio typically used to spectroscopically measure plasma densities is the He-like $\left[1 s^{2}\left({ }^{1} S_{0}\right)-1 s 2 p\left({ }^{1} P_{1}\right)\right]$ "He $e_{\alpha}$ " resonance to $\left[1 s^{2}\left({ }^{1} S_{0}\right)-1 s 2 p\left({ }^{3} P_{1}\right)\right]$ intercombination "IC" line ratio, " $\alpha$ ". The "RATION" (CRE) " $\alpha$ " line ratio was shown earlier in Figure 21\%. I have compared the spectroscopic and interferometric density profiles for various times during and after the laser heating pulse in Figure 44. This is for the $100 \mu \mathrm{m}$ diameter (100\%) Al microdot plasma heated by $1.0 \times 10^{14} \mathrm{~W} / \mathrm{cm}^{2}$ [Shots $* 8433, * 8459, \# 8460, \# 8464$, and $\# 8465$ ]. The holographic interferometer time frames presented either bracket or occur during the time interval representative of the particular FCXS time frame. 
Figure 44. The electron density profiles based on the measured " $\alpha$ "line intensity ratio analyzed using the "RATION" (CRE) model are compared to true interferometric density (axial) profiles for an Al microdot plasma for (top) 730-980 ps (Shots \#8433 \& \#8459), (upper middle) 950-1200 ps (Shot \#8460), (lower middle) $1450-1700$ ps (Shots $* 8460 \&$ \& 4465 ), and (bottom) 1950-2200 ps (Shot "8465). The " $\alpha$ " line intensity has been analyzed assuming a CRE ionization distribution for both an optically thin and thick $(50 \mu \mathrm{m})$ plasma. 

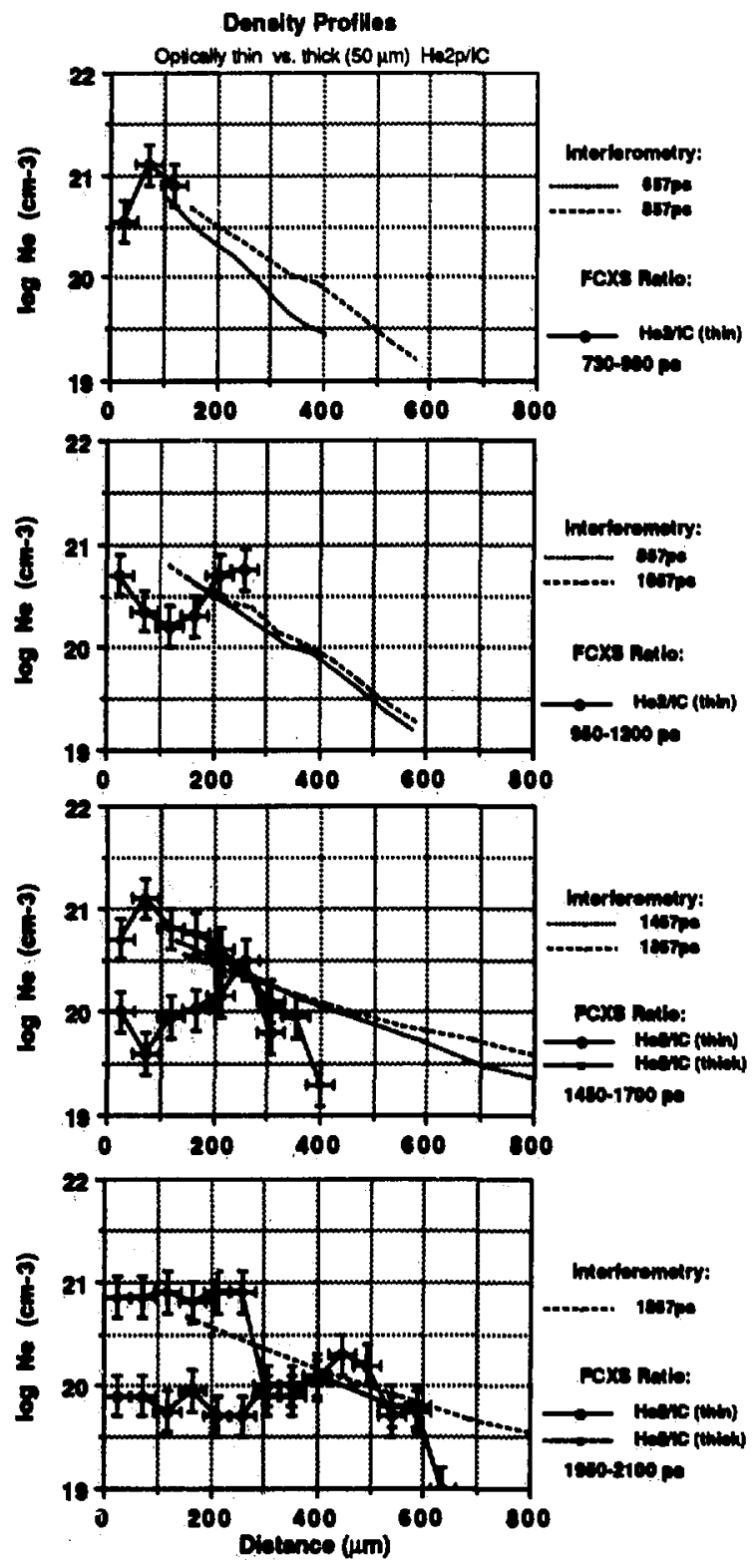
In general, it is difficult to interpret this data due to the large opacity of the He-like resonance line. Analysis of this spectral data is dependent not only on the atomic physics in the model, but also on the detailed treatment of line trapping in the plasma itself. As discussed earlier, opacity has the effect of both trapping excited level poptiations thus leading to a lower observed line intensity (if the plasma is optically thick along the line of sight of the instrument) and also affecting the overall population kinetics of the ions. Therefore, these opacity effects should be kept in mind throughout the remainder of this discussion.

In general, the predicted electron densities deduced from the optically thin line ratios are generally lower, and show very poor agreement with the interferometrically measured densities. Even far out in the plasma plume, beyond $200 \mu \mathrm{m}$ from the target surface, where the plasma should be less affected by opacity problems, the line ratio density is still lower than the actual (interferometric) value. This is consistent with a non-stationary (mostly recombining) plasma as described by several authors such as Boiko et al. (1983). In the CRE analysis, the "IC" line intensity is not wccurately accounted for leading to misinterpretation of the predicted CRE line intensition.

I have also attempted to account for the plasma opacity using the escape factor correction in the "RATION" (CRE) code based on a $50 \mu \mathrm{m}$ plasma size. [Cuble (1987)] For very thick line ratios, the "He $/ \mathrm{IC}$ line ratio versus density plot rolls over and becomes double valued. Thus for a given optically thick line ratio, there may be more than one value of the electron density corresponding to that line ratio. I have selectively chosen one of the density values (based on the monotonically decreasing density profiles measured using interferometry) using the optically thick line intensity ratios as predicted by the "RATION" code. The density profiles derived in this fashion using the optically thick, "RATION" calculation are in 
somewhat better agreement with the actual (interferometric) profiles and are also shown in Figure 44. Obviously, a large number of assumptions have been made and this agreement is likely to be coincidental. For the two early time frames, the observed line intensity ratios (data not presented here) are much larger than the optically thick "RATION" code predictions and casts further suspicion on this particular analysis.

I have also attempted to account for the plasma opacity using the "He $\beta$ " $\left[1 \mathrm{~s}^{2}\left({ }^{1} \mathrm{~S}_{0}\right)-\right.$ $\left.1 \mathrm{~s} 3 \mathrm{p}\left({ }^{1} \mathrm{P}_{1}\right)\right]$ to "IC" line ratio. The "RATION" (CRE) "He $\beta / \mathrm{IC}$ " line ratio was shown earlier in Figure $21 b$. The $(n=3)$ to $(n=1)$ " $L_{\partial}^{\prime} \beta$ " line should be somewhat less optically thick than the $(n=2)$ to $(n=1)$ " $L y_{\alpha}$ " line. Unfortunately, this line ratio is also affected by opacity problems for the observed plasma conditions. Also, the "He $\beta$ /C" line ratio is slightly less density sensitive and therefore less reliable as a density diagnostic. Still, I have derived the electron density profile using this line ratio and compare it to the interferometric density profiles as shown in Figure 45. Again, it is very difficult to note a clear, discernible trend using this data although in general, the line ratio densities appear to be lower than the actual values. This would be consistent with a nonequilibrium recombining plasma as described previously.

Another uncertainty is the contributions to the "IC" intercombination line due to unresolved dielectronic satellite lines "mn", $\left[1 s^{2} 2 p\left(2 P_{3 / 2,1 / 2}\right)-1 s^{2} p^{2}\left(2 S_{1 / 2}\right)\right]$ and "st", $\left[1 \mathrm{~s}^{2} 2 \mathrm{~s}\left(2 \mathrm{~S}_{1 / 2}\right)-1 \mathrm{~s} 2 \mathrm{~s}\left({ }^{3} \mathrm{P}\right) 2 \mathrm{p}\left({ }^{2} \mathrm{P}_{3 / 2,1 / 2}\right)\right]$ at $7.7987 \AA, 7.8022 \AA, 7.8097 \AA$, and $7.8085 \AA$, respectively. [Duston et al. (1983)] The "RATTON" code plots for the "He $\alpha((\mathrm{IC}+\mathrm{mn}+\mathrm{st}) "$ line intensity ratio as a function of the electron density for an optically thin (left) and thick [50 $\mathrm{mm}$ plasma size] (right) were shown in Figure 21c. The contributions due to these satellite lines may be significant at the low electron temperatures characteristic of these plasmas. In Figure 46, I have plotted the density 
Figure 45. The electron density profiles based on the measured "Hep/IC" line intensity ratio analyzed using the "RATION" (CRE) model are compared to true interferometric density (axial) profiles for an Al microdot plasma for (top) 730-980 ps (Shots $\# 8433 \&$ \&459), (upper middle) 950-1200 ps (Shot \#8460), (lower middle) $1450-1700$ ps (Shots $\$ 8460$ \& $\$ 8465$ ), and (bottom) 1950-2200 ps (Shot "8465). The "Heq/ /C" line intensity has been analyzed assuming a CRE ionization distribution for both an optically thin and thick $(50 \mu \mathrm{m})$ plasman. 

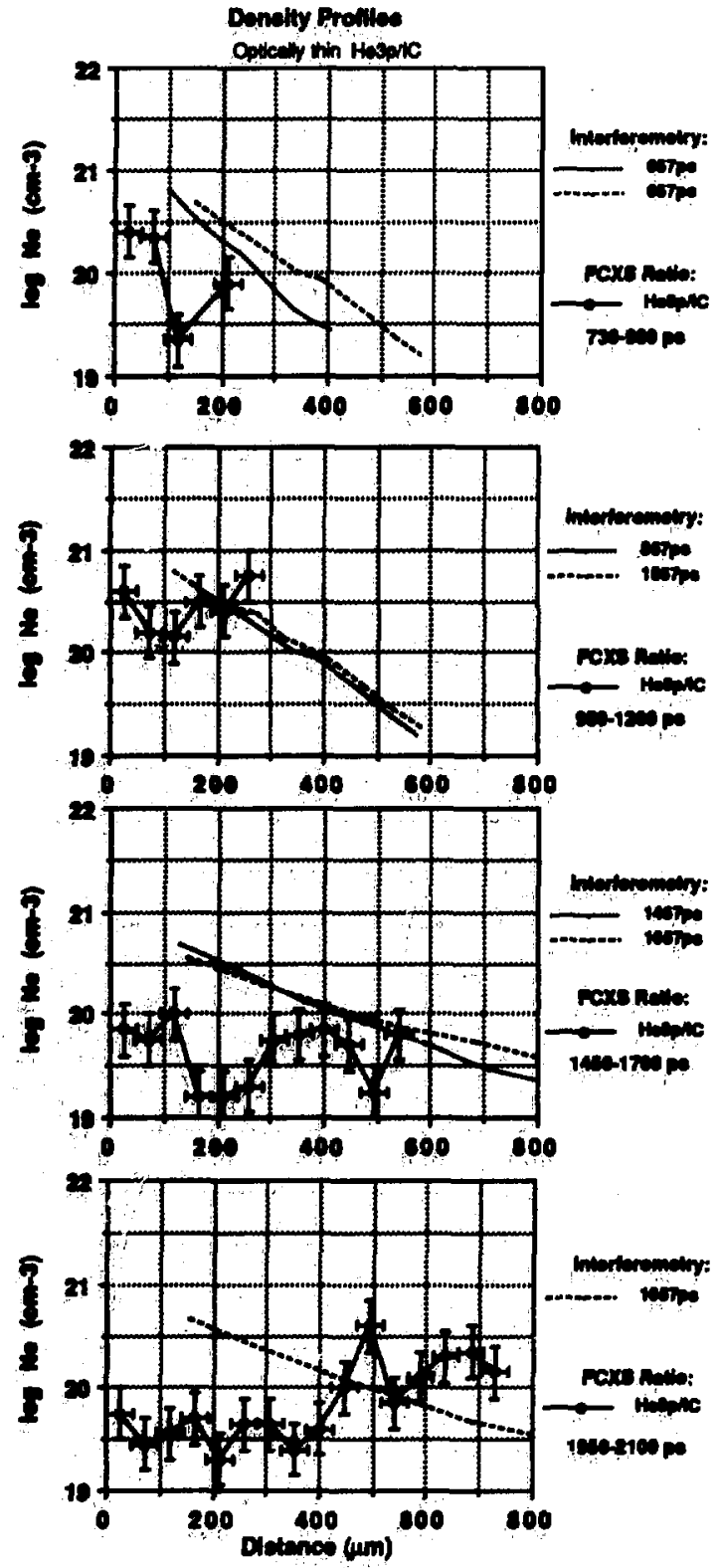

Figure 45 
Figure 46. The eloctron density profiles based on the measured " $\mathrm{He} \alpha / \mathrm{IC}$ " or " $\alpha$ " line intensity ratio and the " $\mathrm{He}_{\alpha} /(\mathrm{IC}+\mathrm{mn}+\mathrm{st})$ " line intensity ratio analyzed using the "RATION" (CRE) model are compared to true interferometric density (axial) profiles for an Al microdot plasma for (top) 730-980 ps (Shots $\# 8433$ \& \#8459), (upper middle) 950-1200 ps (Shot *8460), (lower middle) 1450-1700 ps (Shots $\# 8460$ \& \#8465), and (bottom) 1950-2200 ps (Shot \#8465). The " $\alpha$ " and "Hed $/(\mathrm{C}+\mathrm{mn}+\mathrm{at})$ " line intensity has been analyz:d assuming a CRE ionization distribution and an optically thin plasma. 

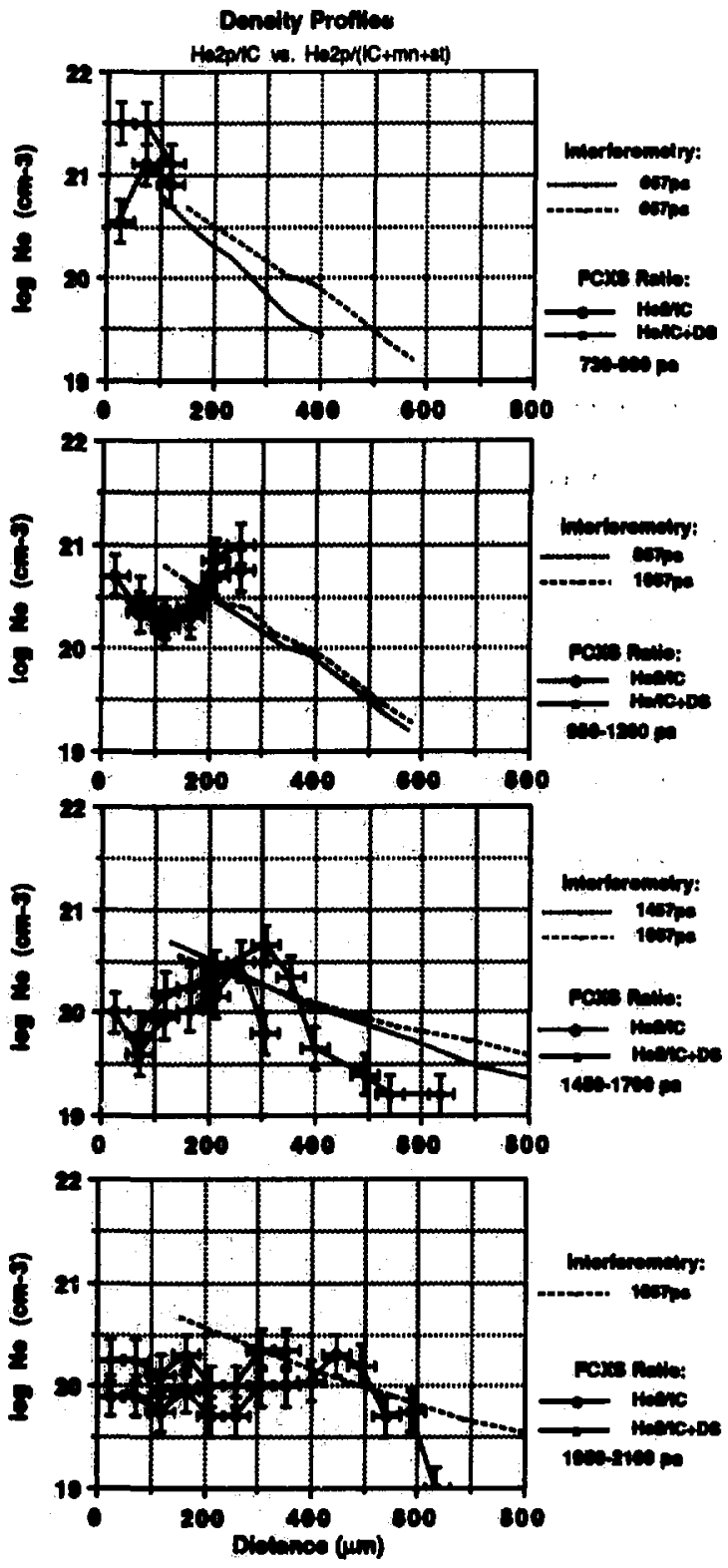
profiles determined from the "RATION" code " $\mathrm{He}_{\mathrm{\alpha}} /(\mathrm{IC}+\mathrm{mn}+\mathrm{st})$ " line ratios against the interferometric densities. Inclusion of the unresolved satellite lines seems to help raise the line ratio densities to better agreement with the actual density profiles. This is just one of many factors that should be considered in future work towards characterizing the dersity dependence of the " $\mathrm{He} / \mathrm{d} / \mathrm{C}$ " line ratio.

\subsubsection{A Quad-Steady State Analysis: Recombination and Excitation Erfects on $\mathbf{K}-$-ihell Lines}

A $10 \% \mathrm{Al}, 90 \%$ graphite target was also studied under the same laser irradiance conditions (approximately $1.0 \times 10^{14} \mathrm{~W} / \mathrm{cm}^{2}$ ). [Shot *8427] The smaller number of Al ions ideally reduces the optical depth of the target by the same amount. Therefore, this target is expected to be approximately $10 \%$ as optically thick as the standund $100 \%$ Al target for the same laser-plasma conditions. In Figure 47, I have

derived the density profile from the " $\alpha$ " line ratio using the optically thin "RATION" calculation. The frame times are $730-980 \mathrm{ps}, 1230-1480 \mathrm{ps}$, and $1730-1980$ ps corresponding to sample times both during and after the $1.0 \mathrm{~ns}$ heating laser pulse. These line ratio profiles fall consistently lower than the corresponding interferometry profiles as would be expected for a non-stationary plasma. This difference is most likely due to the CRE calculation not fully accounting for the preferential recombination processes feeding of the ${ }^{3} \mathrm{P}$ excited level configuration and therefore underestimating the "IC" line intensity. If the measured "IC" line intensity is large (due to recombinations) relative to the CRE model predictions, then a lower electron density will be inferred by the CRE model for a given electron " $\alpha$ " line ratio. 
Figure 47. The electron density profiles based on the measured " $\alpha$ " line intensity ratio analyzed using the "RATION" (CRE) model are compared to true interferometric density (axial) profiles for an Al microdot plasma for a 10\% Al plasma at $1.0 \times 10^{14} \mathrm{~W} / \mathrm{cm}^{2}$ for (top) $730-980 \mathrm{ps}$, (middle) $1230-1457 \mathrm{ps,} \mathrm{and}$ (bottom) 1730-1980 ps (Shot \#8427). The " $\alpha$ " line intensity has been analyzed assuming a CRE ionization distribution and an optically thin plasma. 

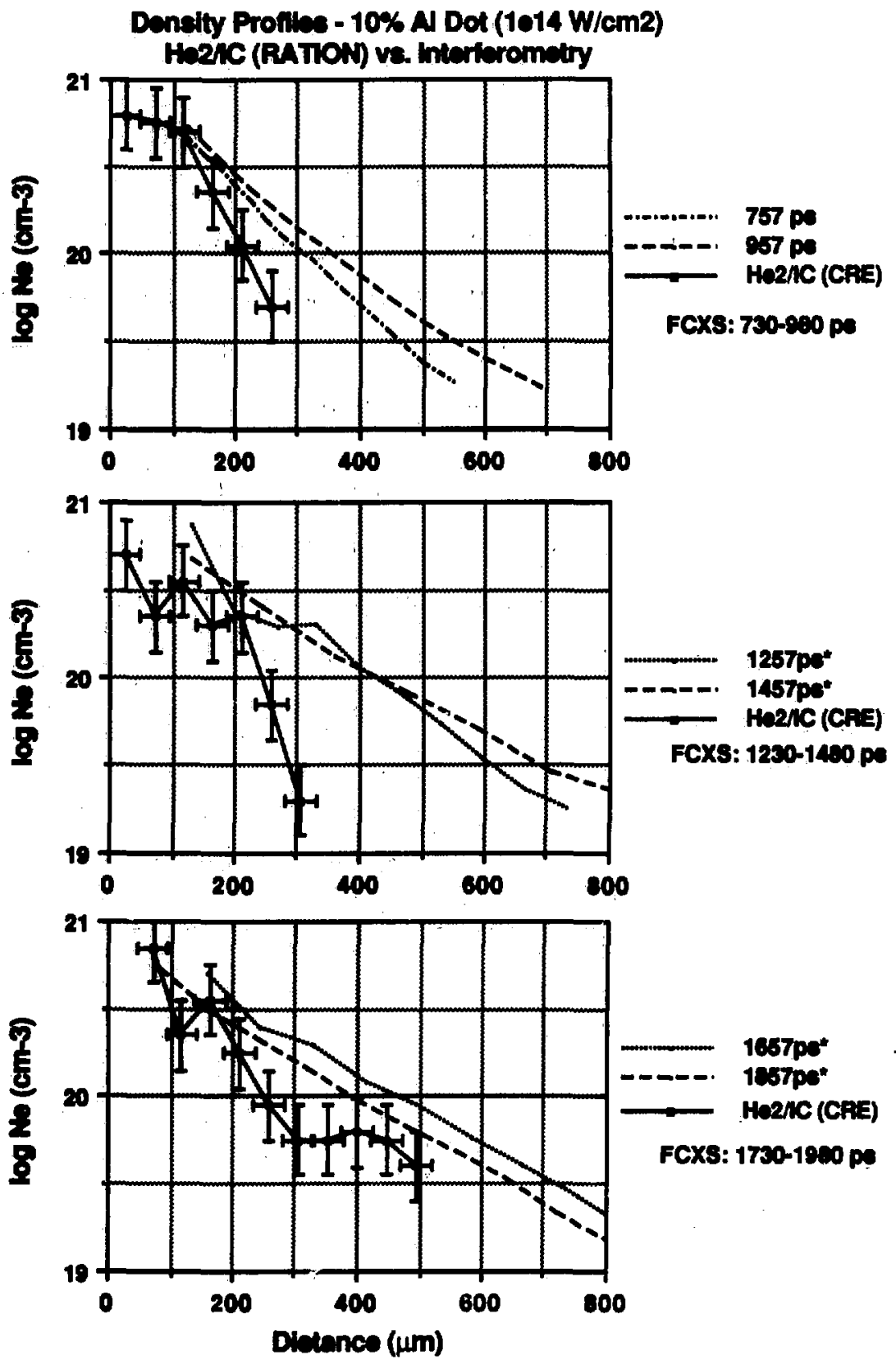
Figure 48. The electron density profiles based on the measured " $\alpha$ " line intensity ratio analyzed using the quasi-steady state $\mathrm{K}$-shell model are compared to the true interferometric density (axia) profiles for an $\mathrm{Al}$ microdot plasma for $210 \% \mathrm{Al}$ plasma at $1.0 \times 10^{14} \mathrm{~W} / \mathrm{cm}^{2}$ for (top) $730-980 \mathrm{ps,} \mathrm{(middle)} 1230-1457 \mathrm{ps}$, and (bottom) 1730-1980 ps (Shot \#8427). The " $\alpha$ " line intensity has been analyzed assuming either a purely recombining plasma or a purely direct excitation populated plasma. The "recombining plasma" line intensity ratios predict densities that are in excellent agreement with holographic interferometry. 

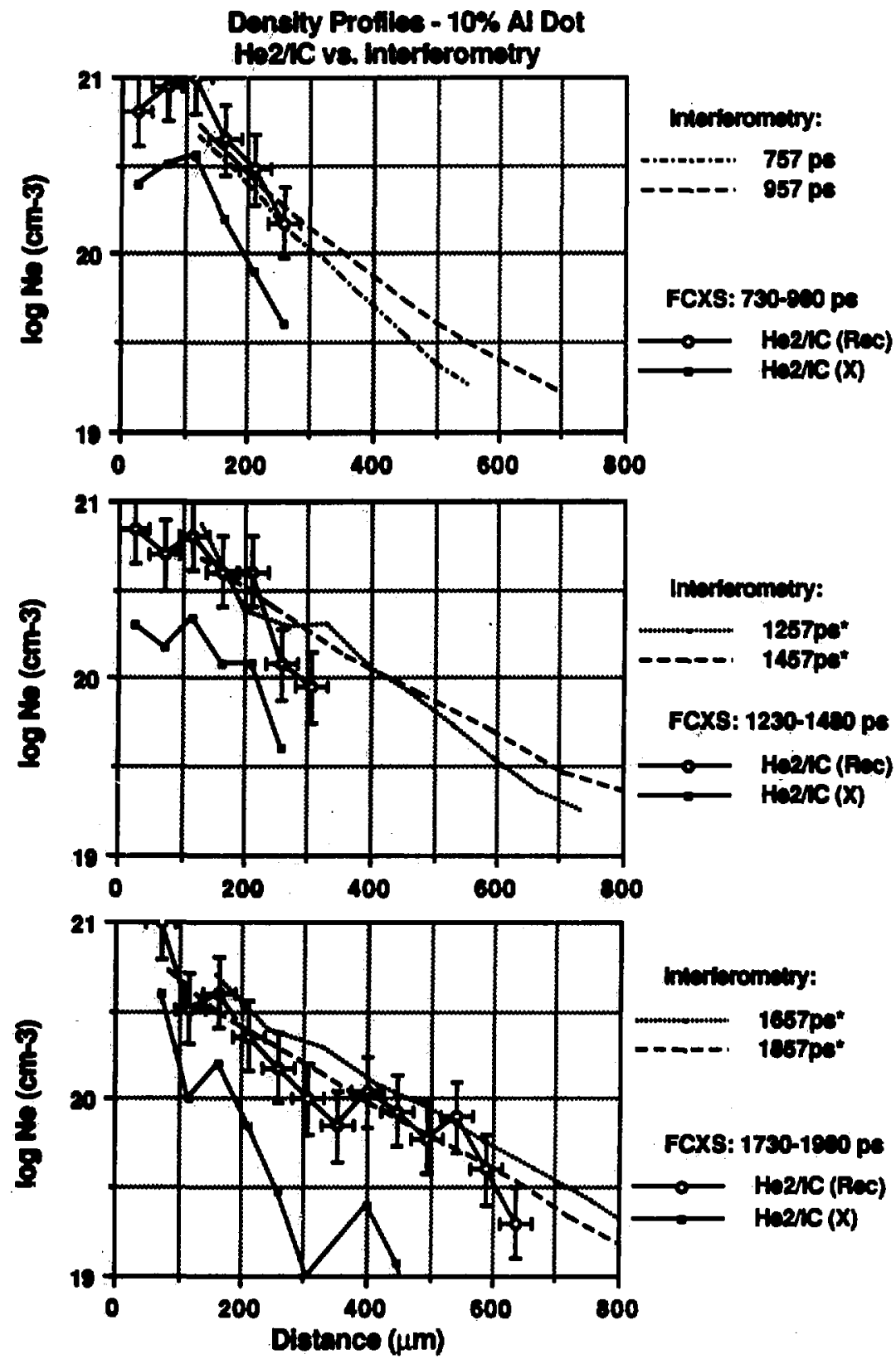

Figure 48 
calculated for the purely recombination and purely direct excitation cases (See Figure 27) to this $10 \%$ Al data. [W. Goldstein anc' A. Osterheld (1988)] The pure excitation model deduced densities fall far below the experimental (interferometric) electron densities, even lower than the CRE values. This is not surprising since the CRE model is a sum of many atomic processes (including excitation, recombination, and ionization) based on an eqvilibrium charge state distribution. Despite the severe disagreement, this does indicate that the CRE " $\alpha$ " line ratio analyses are accurate and not compromised by any systematic errors.

For the recombination model predictions, there is very good agreement between the interferometric and the " $\alpha$ " line ratio determined electron density profiles for all three FCXS time frames. For the two later sample times, the agreement with a purely recombining model is ressonable as there is no additional heating due to laser energy absorption. As the electron temperature decreases, the recombination rate increases and the plasma recombines. There appears to be good agreement while the heating laser is still on, out away from the target surface $(2200 \mu \mathrm{m})$. This may indicate that recombination procesces are dominant far out in the plasma plume even when the laser is on. This is not well understood and remains open to speculation. Closer in towards the target surface, the purely recombination model fails for a number of reasons. First, it is likely that excitation and perhaps ionization processes are important close in during the heating pulse. Also, line opacity effects may play a role in this plasma. In order for this quasi-steady state analysis to fully characterize this phenomena, additional improvements to the model are required. The $\mathrm{H}$-like and Li-like systems should be included as should their corresponding recombination and ionization proceseses. Better 3-body recombination rates may be needed. Also, opacity corrections need to be included into the quasi-steady state model.

One difficulty with this analysis is that therc was no independent free-bound 
recombination continuum measurement of the electron temperature profile. Therefore, I have assume that the $10 \%$ and $100 \%$ targets behave similarly and I have used the temperature profiles determined for the $100 \%$ Al target to interpret the " $\alpha$ " line ratio. This is not a significant factor as the " $\alpha$ " line ratio is relatively independent of the electron temperature.

Another factor is that only a small number of $10 \% \mathrm{Al}$ targets were studied and therefore a complete set of interferometric density profiles were not measured for all times during and after the heating laser pulse. Therefore for the later times, I have compared the " $\alpha$ " line ratio densities to the $100 \% \mathrm{Al}$ interferometric density profiles. It should be noted that the interferometric density profiles that were measured for the $10 \%$ target (at $357,557,757$, and $957 \mathrm{ps)}$ ) are in very close agreement with interferometric density profiles for the $100 \%$ Al plasma. Therefore, this analysis should be valid.

\subsection{Measurement of Inverted High-n Resonance Line Intenaity Ratios in He-like Ion}

Another very interesting observation is the "inverted" relative intensities of the high-n He-like resonance line intensities measured at late times after the heating laser is off, and far out in the plasma plume. These lines correspond to the $\left.1 \operatorname{snp}^{1}{ }^{1} \mathrm{P}_{1}\right)$ excited state levels which decay radiatively to the $1 s^{2}\left({ }^{1} \mathrm{~S}_{0}\right)$ ground state. Instead of the resonance lines falling off in intensity with increasing values of $n$, as might be expected for pure direct excitation from the ground state, the high-n resonance line intensities appear "inverted". The higher-n lines (such as the n=4 or 5) are stronger in intensity that the lower- $n$ lines (such as the $n=3$ ). This effect is due to the nature 
of the plasma population linetics for these late time coronal plasmas. The primary mechanism driving the population level kinetics of the ions is recombination processes, particularly 3-body recombination which preferentially feeds the high-n levels. This effect was described in section 4.2 and is predicted by the recombining quasi-steudy state K-shell model calculations of A. Osterheld and W. Goldstein. Because this effect is due to recombination processes, these inverted line intensities are directly related to the electron density of the plasma.

I have examined two particular Al microdot target shots, \#8455 and $\# 8465$, at 3.5 $\times 10^{13} \mathrm{~W} / \mathrm{cm}^{2}$ and $1.0 \times 10^{14} \mathrm{~W} / \mathrm{cm}^{2}$, respectively. Data from selected time frumes for these two shots are shown in Figures 49a and 49b for Shot 48455 , at 1230-1480 and 1730-1980 ps, respectively, and in Figure 49c for Shot 48465 at 1450-1700 ps. These datn were measured using the FCXS instrument and I have shown sets of densitometer scans representing the $x$-ray spectra emitted by $50 \mu \mathrm{m}$ wide regions of plasma centered at various distances from the target surface. These data show the inverted high-n resonance line intensities in the He-like spectra and also in the H-like series of lines although I have not compared the $\mathrm{H}$-like line ratios with theory in the present work. In particular, the high-n He-like resonance line ratios such as the He-like $\left[1 s^{2}\left({ }^{1} S_{0}\right)-1 s 4 p\left({ }^{1} P_{1}\right)\right]$ "He $\gamma^{\prime}$ to He-like $\left[1 s^{2}\left({ }^{1} S_{0}\right)-1 s 3 p\left({ }^{1} P_{1}\right)\right] ~ " H e e^{\prime \prime}$ line ratio and the He-like $\left[1 s^{2}\left({ }^{1} S_{0}\right)-1 s 5 p\left({ }^{1} P_{1}\right)\right]$ "Hes" to He-like $\left[1 s^{2}\left({ }^{1} S_{0}\right)-\right.$ 183p $\left({ }^{1} P_{1}\right)$ ] "Hep" resonance line ratios show this inverted behavior in which the higher-n resonance line is stronger than the lower-n line. The electron density profiles were determined independently and simultaneously via holographic interferometry. Axial electron densities have been used in the present analysis. The electron temperature profiles have been measured from the slope of the H-like free-bound recombination continuum using the FCXS data from similar target shots [Shots $\# 8464\left(-1.0 \times 10^{14} \mathrm{~W} / \mathrm{cm}^{2}\right)$ and $\# 8466\left(-4.0 \times 10^{13} \mathrm{~W} / \mathrm{cm}^{2}\right)$ ]. For these 
Figure 49. Simultaneous space and time resolved (FCXS) Al spectra exhibit intensity inversions in the high-n resonance lines (He-like and $H$-like) at late times. Densitometer scans showing the aluminum $\mathrm{K}$-shell spectra emitted from $50 \mu \mathrm{m}$ wide regions of plasma at various distances from the target surface for a) Shot $\# 8455$ (1230-1480 ps), b) Shot $\# 8455$ (1730-1980 ps), and c) Shot $\# 8465$ (1450-1700 ps). 


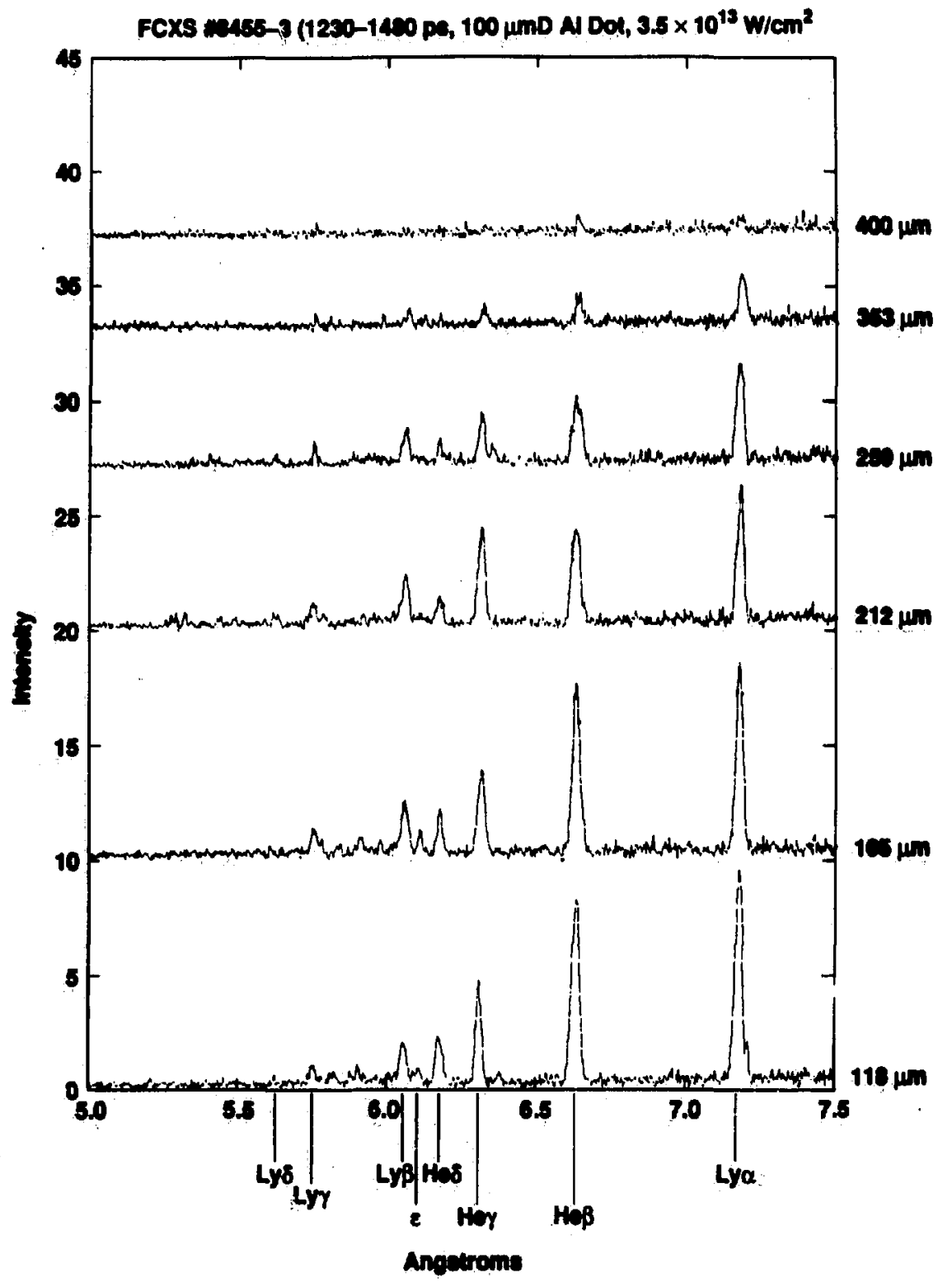




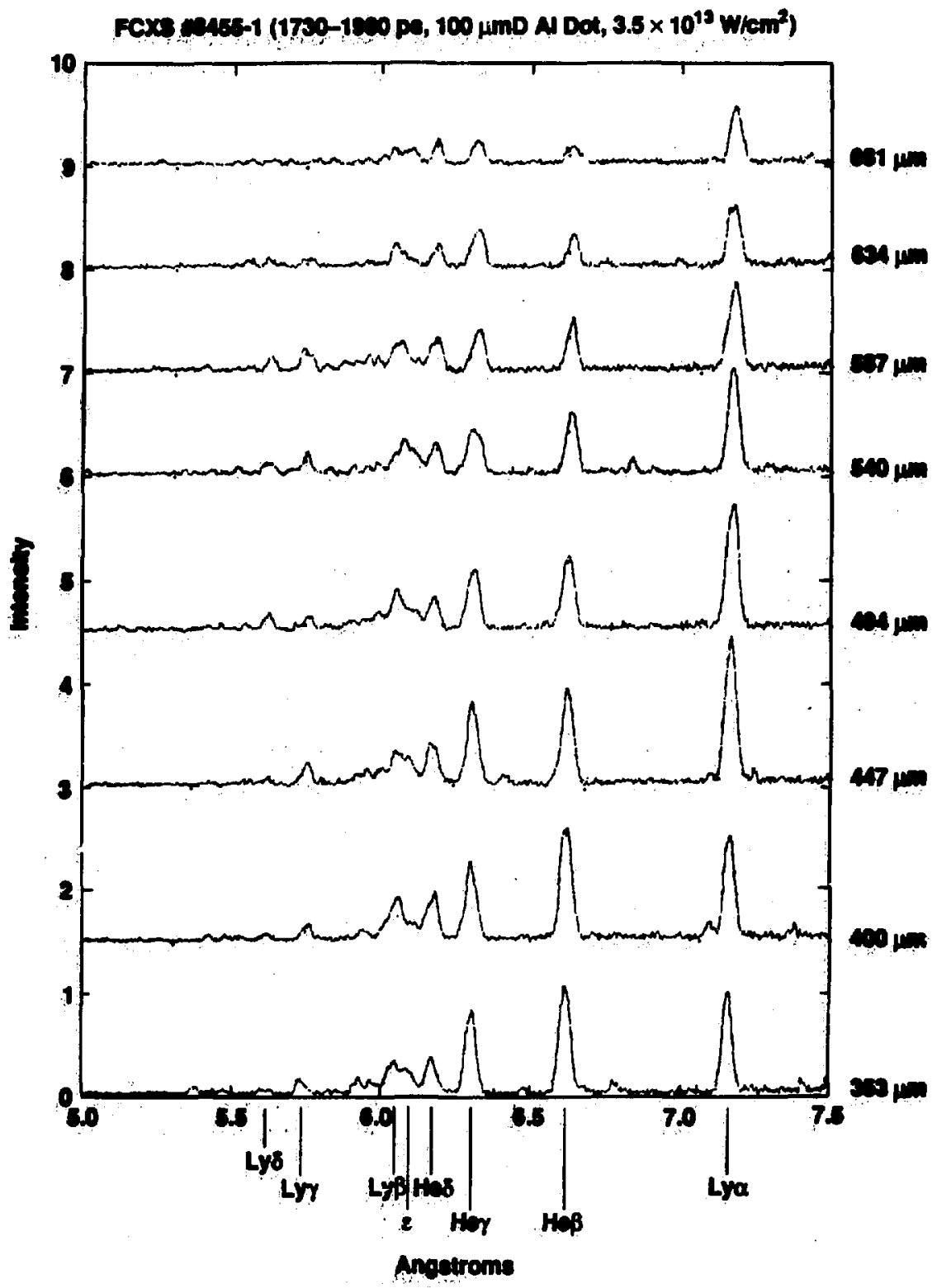

Figure 49b 


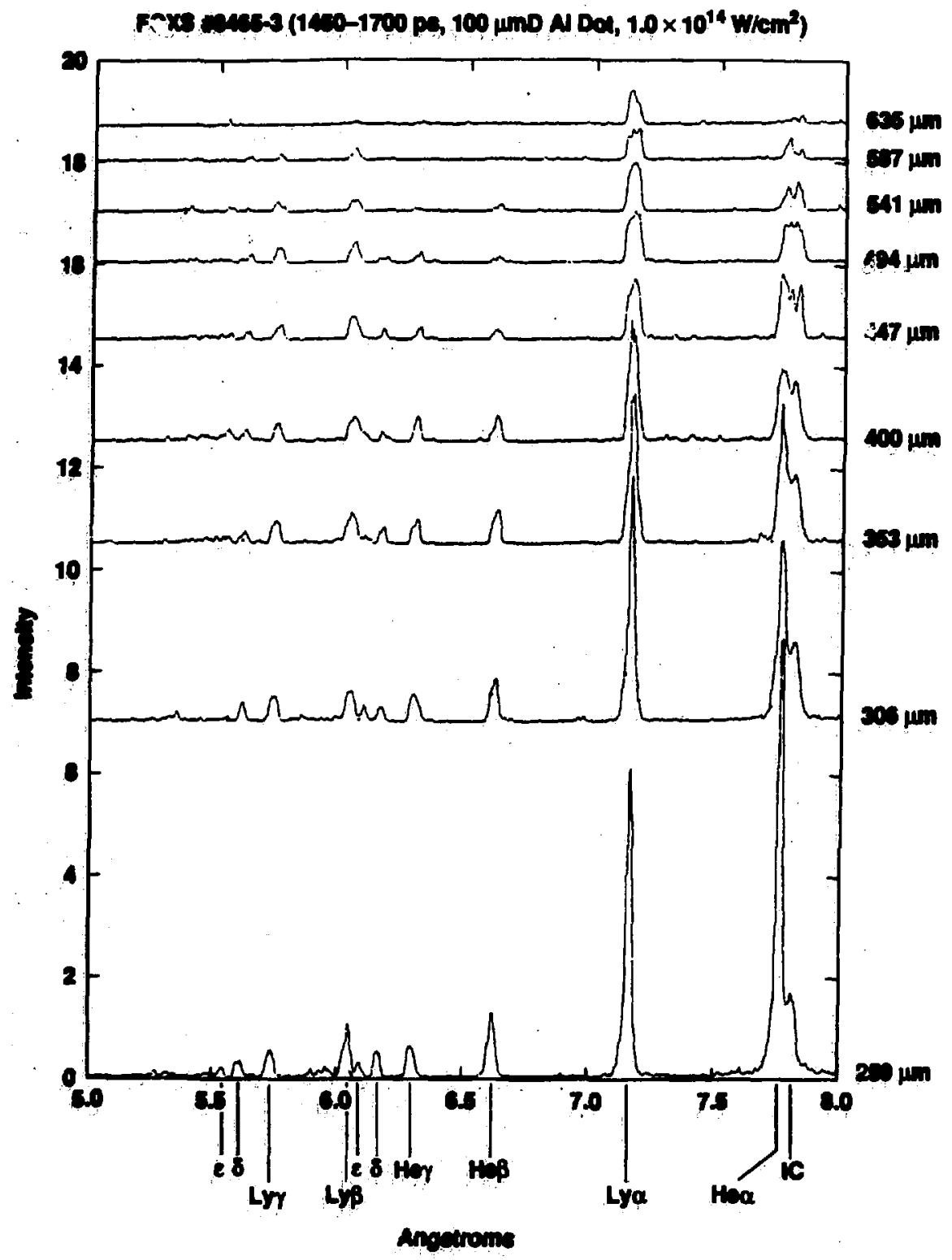

Figure $49 c$ 
late times and large plasma distances (2200 $\mu \mathrm{m}$ out into the plasma corora), the temperature profile is relatively flat ranging from $60-100 \mathrm{eV}$ for the $1.0 \times 10^{14}$ $\mathrm{W} / \mathrm{cm}^{2}$ shots, and approximately $50-90 \mathrm{eV}$ for the lower intensity shots.

The experimentally measured and the theoretically calculated (quasi-steady state recombination model) "He$/ \mathrm{He}_{\beta}$ " (top) and " $\mathrm{He} / \mathrm{He}_{\beta}$ " (bottom) line intensity ratios have been plotted versus the electron density in Figures 50n (Shot \#8455; 1230-1480 ps), $50 \mathrm{~b}$ (Shot $\$ 8455 ; 1730-1980 \mathrm{ps}$ ), and 50c (Shot *8465; 1450-1700 ps). The predicted line invensity plots have been shown for three different electron temperatures: 50,100 , and $200 \mathrm{eV}$. There is good qualitative agreement between experiment and theory. The datu shows the sume "humped" profile which is predicted by theory, withough the datu falls some what lower than predicted (peaking at a bwer electron density) even for the $50 \mathrm{eV}$ curve. The actual electron temperatures are somewhat higher $(75-100 \mathrm{eV})$ than $50 \mathrm{eV}$ and suggests minor problems with the calculations. There may be some uncertainty in the 3-body recombination rates (derived from detailed belance with Lotz formula collisional ionization rases) used in the quasi-steady stave model. In fact, this could indicate a method for measuring the 3-body recombination rates based on measuring the high-n resonance lines from a recombining laser-produced plasma. The "humper' line intensity ratio (as a function of density) is likely due to the higher-n levels being rloser to Local Thermodynamic Equilibrium (LTE) than the lower lying, lower-n level. Thesefore, there is an optimum electron density in which the high-n to low-n line ratio peaks, somewhere between the LTE limit where both kevels are populated statistically, and where the 3-body recombination rate (varies $\propto n_{e}^{2}$ ) becomes a less important populating process. The "humped" profile is described in greater detail in section 4.2.

The inverted high-t le-like resonance line intensities also suggest population 
Figure 50. The quasi-steady state recombiring Al plasm model predicts line intensity inversions between the high-n He-like resonance lines as seen in the FCXS data. The theoretically predicied versus experimentally measured (top) "He//He $\beta$ " and (bottom) "Heg/He presented for a) Shot $* 8455(1230-1480 \mathrm{ps})$, b) Shot $\# 8455$ (1730-1980 ps), and c) Shot $\$ 8465$ (1450-1700 ps). Free-bound recombination continuum temperatures (not shown) and interferometric densities have been used in this analysis. 

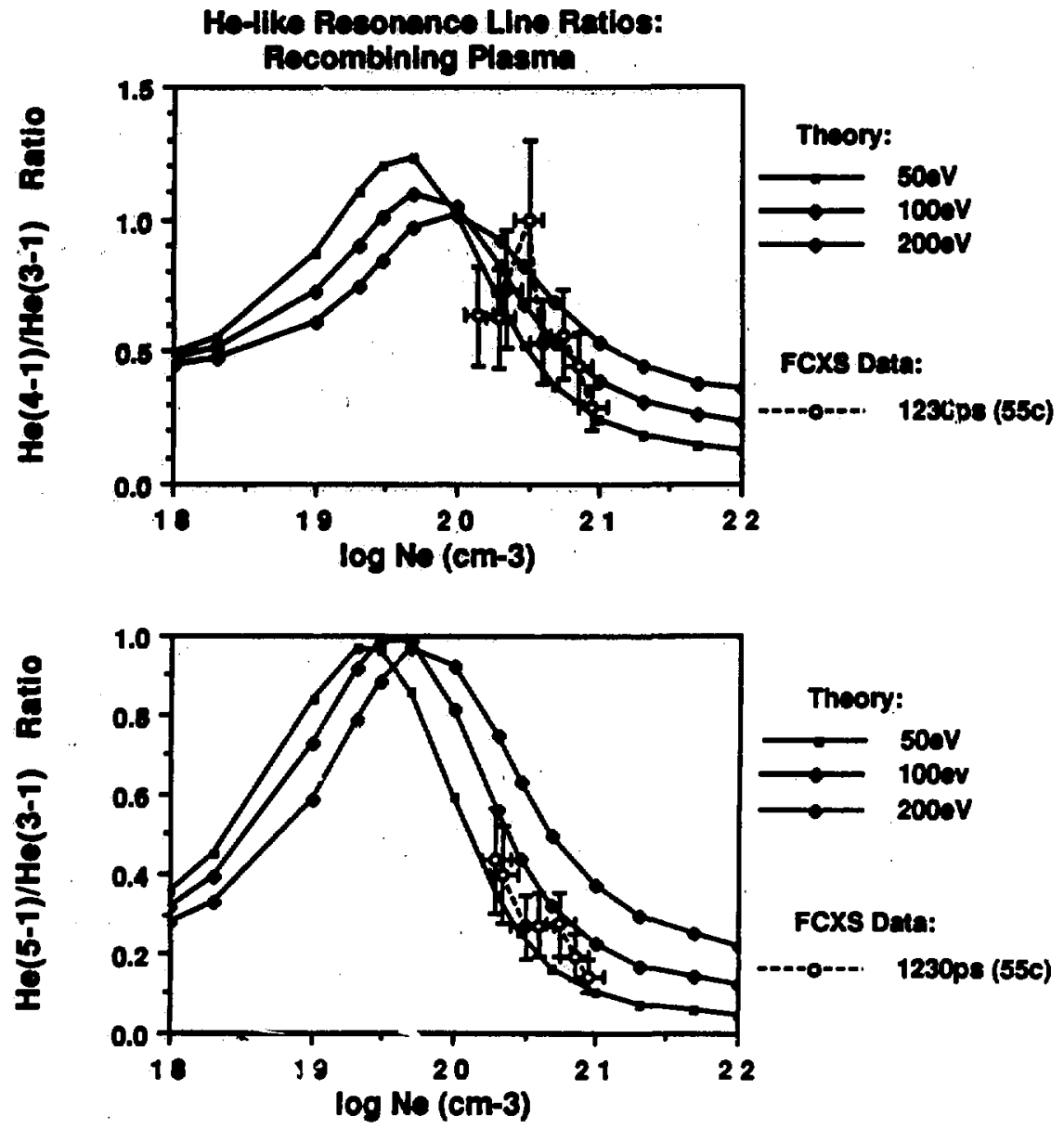

Figure 50a 

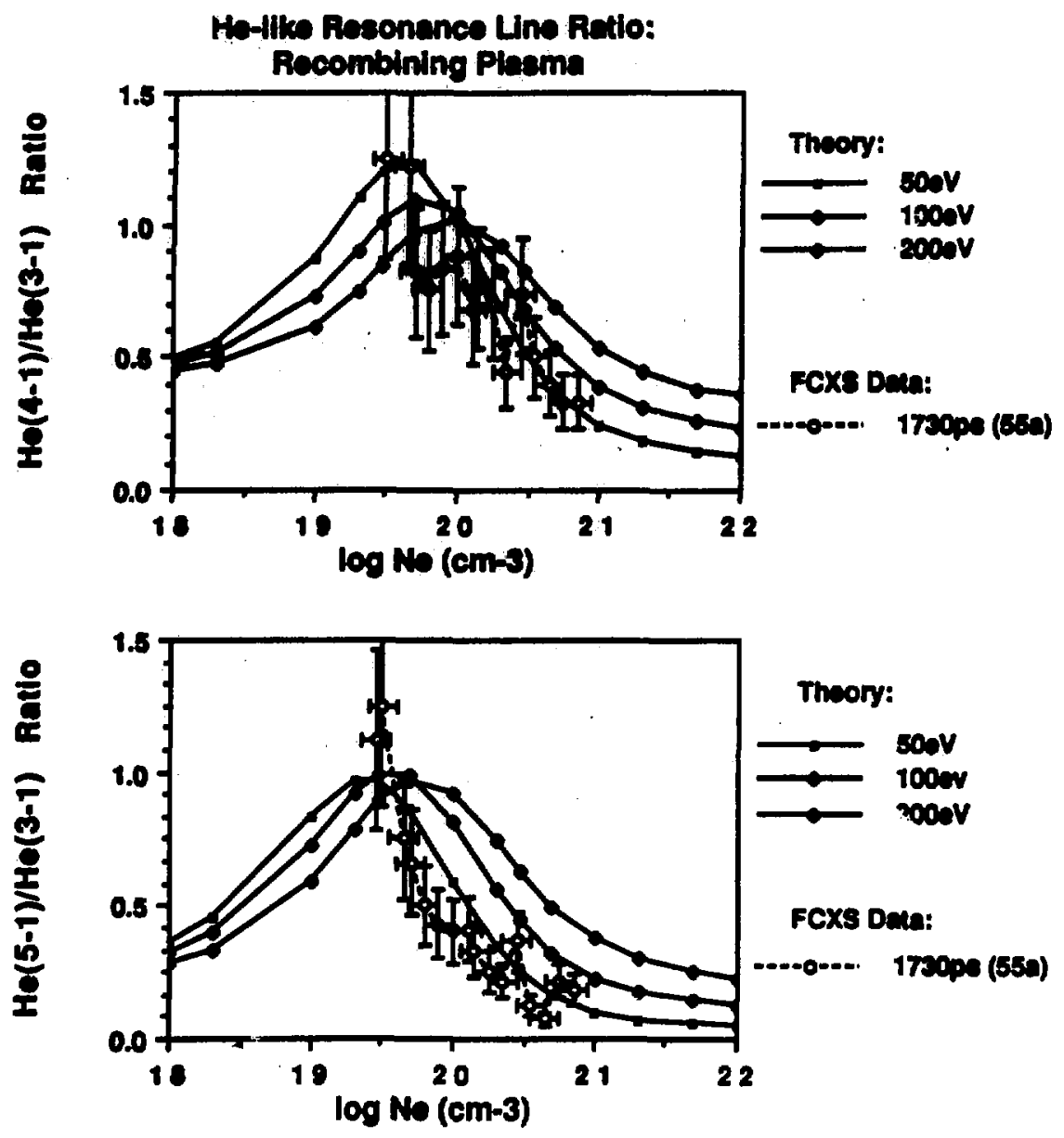

Figure 50b 

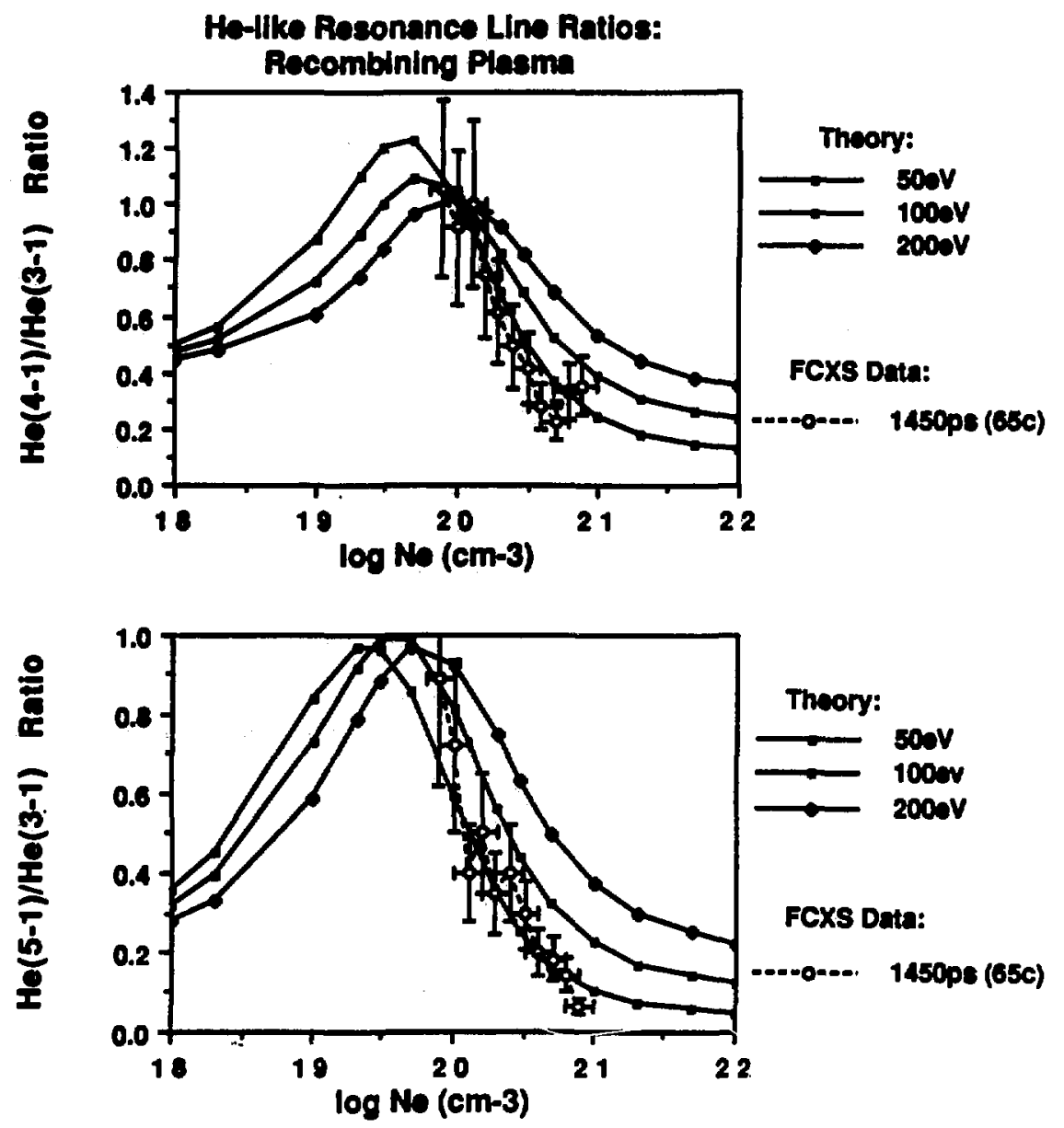

FIgure 50c 
inversions between the metastable $n=4$ and $n=5$ level manifolds to the $n=3$

$\left[1 \mathrm{~s} 3 \mathrm{p}\left({ }^{1} \mathrm{P}_{1}\right)\right]$ level which rapidly radiatively decay back to the He-like ground state.

These population inversions may be in transitions such as the $\left[1 \mathrm{~s} 3 \mathrm{p}\left({ }^{1} \mathrm{P}_{1}\right)-\right.$ $\left.1 \mathrm{~s} 5 \mathrm{~d}\left({ }^{1} \mathrm{D}_{2}\right)\right]$ and the $\left[1 \mathrm{~s} 3 \mathrm{p}\left({ }^{1} \mathrm{P}_{1}\right)-1 \mathrm{~s} 4 \mathrm{~d}\left({ }^{1} \mathrm{D}_{2}\right)\right]$ transitions. For $\mathrm{Al}$, these transitions are of order $85 \AA$ for the $3 \mathrm{p}-5 \mathrm{~d}$ transition and $123 \AA$ for the $3 \mathrm{p}-4 \mathrm{~d}$ transition. The magnitudes of these population inversion is presently being analyzed and will be reported in a separate report. [Goldstein et al. (1988)] These potential short wavelength lasing transitions are of considerable interest for several reasons. First, transitions between He-like ( $2 p-3 d)$ and $\mathrm{H}$-like ( $2 \mathrm{p}-3 \mathrm{~d}$ and $2 \mathrm{p}-4 \mathrm{~d})$ correspond to soft $x$-ray transitions of less than $40 \AA$, below the carbc $\cdot K_{-\alpha}$ absorption edge. The data recorded from these experiments provides a clear space and time resolved measurement of the population inversion as represented by the inverted resonance line intensities and based on the quasi-steady state $\mathrm{Al}$ model. The plasma has been fully characterized with independently determined space and time resolved density and temperature profiles providing an excellent data base for the testing and development of theoretical atomic models required to calculate the population inversions in the laser medium. Overall, it is clear that the quasi-steady state model, presented in Chapter 4 and used in the present analysis, does a good job in simulating the complex population kinetics governing the He-like excited level including recombination processes, collisional $(\Delta n=0)$ mixing, radiative cascades, and in representing the detailed excited state level structure for these ions. The high-n H-like resonance lines also show this same inverted line intensity ratio behavior and will be studied using the completed (bare, $\mathrm{H}-, \mathrm{He}$, and Li-like) quasi-steady state K-shell model at a later time. 


\subsection{Spatially Resolved, Time-Integrated Measurements}

I have also analyzed spatially resolved, time-integrated data for the same Al microdot shots. The measured He-like resonance to intercombination line ratio was interpreted based on $\triangle$ CRE analysis to determine the apparent time-integrated electron density profile and is shown in Figure 51(top). Because this is time-integrated data and there was no independent time-integ $\mathrm{g}_{\mathrm{k}}$.ed temperature measurement, the "He $\mathrm{H}_{\alpha} \mathrm{IC}$ " line ratio was interpreted using "average" electron temperatures of 200,500 , and $700 \mathrm{eV}$. This type of procedure has been used repeatedly in previous spectroscopic studies of laser-produced plasmas. The electron density profile determined from this time-integrated data is much lower than any of the density profiles measured from the time resolved data. This is likely due to the fact that the resonance and intercombination line intensities (emissivities) have completely different spatial and temporal histories as clearly shown in the FCXS data. The resulting density profile determined from this time-integrated data is non-physical and should not be used.

The spatially resolved, time-integrated spectra has also been used to derive time-integrated temperature profiles as shown in Figure 51(bottom). Here, I present temperatures determined from the "He $\beta / y_{\beta}$ " resonance ratio and the "Ly ${ }_{\alpha} /\left({ }^{1} D_{2}\right)$ satellite", and "Hed/jkl satellite" line ratios. I have used the optically thin "RATION" (CRE) calculation and have used the high and low "average" density values from Figure 51(top) to help analyze this data. Again, this is a very common practice in previous laser-plasma characterization work. These line ratio temperatures are much higher than the time resolved values. This is not surprising since the time-integrated line intensities represent a wide range of plasma conditions (temperature and density gradients). As noted previously, most of the line emission 
Figure 51. Spatially resolved, time integrated spectra provide inaccurate line ratio inferred (top) densities (at 200,500, and $700 \mathrm{eV}$ ) and (bottom) temperatures (high and low estimated densities). The "RATION" (CRE) model has been used to analyze this data (Shot "8433). Because this data is time integrated, the line intensity ratios have been analyzed based on a range of electron temperatures or densities. This analysis is typical of standard line ratio plasma diagnostic methods used in previous laser-plasma characterization work. 
TImo Integrated/Space Recolved Denslty Profile

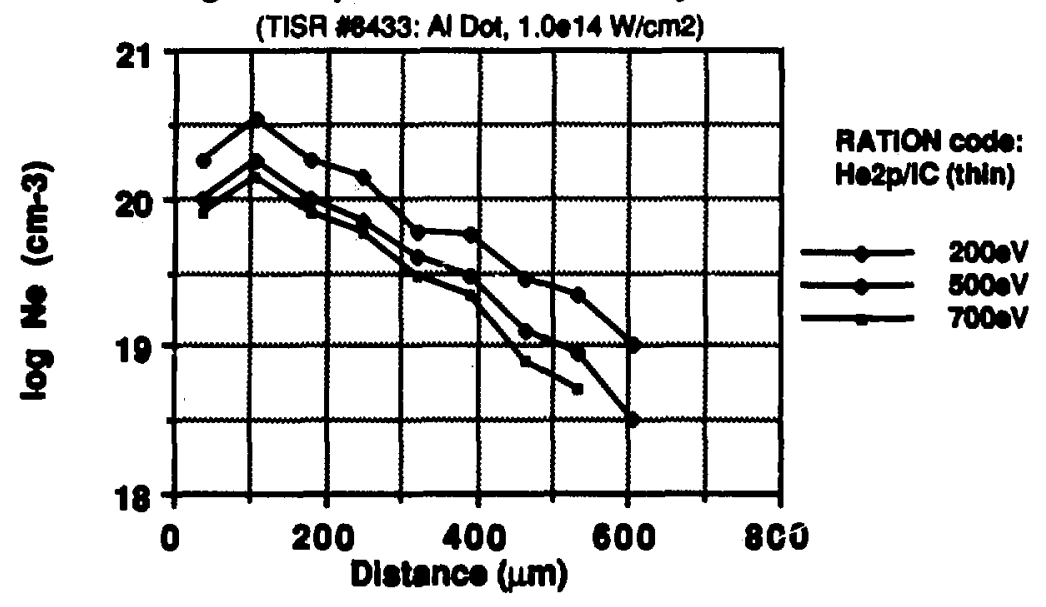

Time Integratod/Space Resolved Temperature Proflle

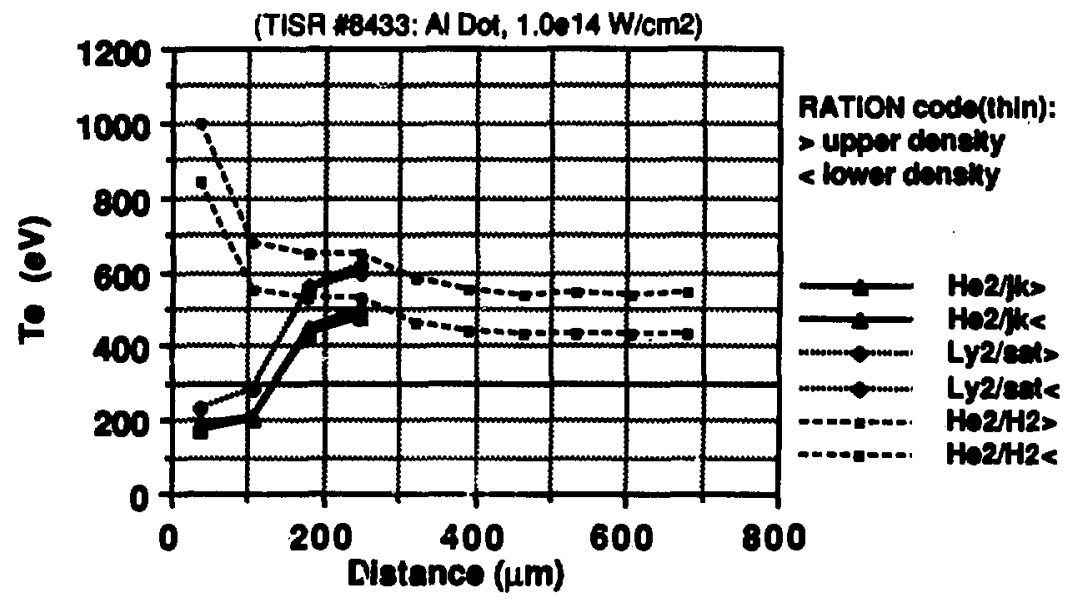

FIgure 51 
observed at distances greater than $100 \mu \mathrm{m}$ from the target surface is emitted after the laser heating pulse when the plasma is predominantly recombining. Thus previous spatially resolved, time-integrated spectroscopic measurements of coronal temperature and density profiles may be in error.

\subsection{Summary}

This work has revealed many new and $7 x c i t i n g$ areas in the atomic physics of highly stripped ions in laser-produced plasmas. First, simultaneously space and time resolved K-shell emission spectra have been measured from Al laser-produced plasmas under a range of different plasma conditions. These plasma have been well characterized using simultaneous, and fairly model-indpendent techniques: multi-frame holographic interferometry to measure the electron density profiles and the slope of the H-like free-bound recombination continuum, measured using the new FCXS instrument, to determine the electron temperature profiles.

Since these laser-plasmas are well characterized, they allow for clear tests of available theoretical models. For instance, two dimensional "LASNEX" hydrodynamic simulations have been compared to the new experimental data. The measured temperature profile during the heating laser pulse is significantly lower than the calculated temperature profile and suggests shortcomings in the hydrodynamic simulation and possibly in understanding the actual laser light absorption process. For later times, both the electron densities and temperatures computed by the "LASNEX" simulation are in reasonably good agreement with experiment, although somewhat lower and predicting less spatial structure thun measured. This work and future experiments of this type should provide an ideal method understand the complex physics of laser-plasma interactions.

The precise population kinetics governing the spectral line intensities have also 
been tested. The nonequilibrium ionization distribution clearly affects the atomic physics of the ions. Atomic models based on an equilibrium charge state distribution, such as the "RATION" (CRE) model, fail to account for the non-stationary atomic processes which govern the physics of these plasmas. This failure has been demonstrated in a number of ways using different line intensity ratios. The CRE He-like to $\mathrm{H}$-like resonance line intensity ratio, which is often used to deduce the electron temperature, clearly fails. The laser-produced plasmas are highly overstripped relatively to the electron temperature and density based on a CRE ionization distribution. The CRE model fails to account for the recombination processes which preferentially feed the "IC" line and therefore, the CRE model fails to predict the sctual density dependence of the" $\mathrm{He}_{\mathbf{d}} / \mathrm{IC}$ " line intensity ratio. During the heating laser pulse and near the target surface, plasma opacity effects introduce uncertainties in the measured data and prevent a clear test of the CRE model. The high-n He-like resonance lines at late times (after the laser is off) and far out into the coronal plasma, clearly exhibit a non-stationary behavior which can not be calculated using the CRE analysis. Only, the CRE "Ly $/\left({ }^{1} D_{2}\right)$ satellite" line intensity ratio seems to show the proper temperature dependence (agreement with free-bound temperature measurements) during the heating laser pulse. During the heating laser pulse and near the target surface, collisional excitation processes appear to be dominant and since both lines originate from approximately the same 1s H-like ground state, the CRE analysis appears to succeed. For later times and further out into the plasma plume, the CRE line ratio analysis fails because the H-like resonance lines are strongly $: \mathcal{i}$ via recombination processes.

The density dependence of various He-like spectral lines are successfilltv predicted using a new, quasi-steady state Al K-shell model. These include the " $\mathrm{He}_{\alpha} / \mathrm{IC}$ " line intensity ratio and the "inverted" high-n resonance line intensities. The quasi-steady 
state model is much different than the CRE model as it is not based on an equilibrium ionization distribution. Instead, the quasi-steady state model treats the relative charge state fractions as independent, free variables along with the electron temperature and density. Therefore, the model allows for the simultaneous and systematic analysis of several spectral line intensities (from various ionization stages) to determine the relative charge state distributions and the plasma temperature and density. Although this new quasi-steady state model is currently being refined, these Al He-like calculations have shown great promise in expunding our knowledge of the physics of these laser-plaumus. Further comparisons with the completed atomic model should be equally enlightening. Thus, these experiments have provide a new avenue to investigave the detailed atomic phyaics processes controlling the population kinetics in these laser-produced plasmas. 


\section{Chapter 6. L-Shell Spectroscony Experiments}

As described earlier, the measurement of the characteristic spectral signature produced by high-powered laser-produced plasmas is a direct, nonperturbing method to probe the local plasma conditions as well as providing detailed emission spectra to develop and test theoretical atomic models. The L-shell spectra comes from high-Z, iors that have been stripped down to fluorine-like, neon-like, sodium-like, etc. The physics of the L-shell $x$-ray emission spectra has been the focus of recent studies towards the development of short wavelength lasing schemes as well as diagnostics for understanding highly ionized plasmas in solar flares [for example, Smith et al. (1985)] and tokamaks [for example, Beiersdofer et al. (1987)]. There have been several studies to measure the emission spectra of these short-lived, high-density laser-pmduced plasmas. Some of the early spectroscopic work on L-shell spectra emitted by laser produced plasmas include studies of zinc [Nagel et al. (1974)], zirconium [Burkhalter et al. (1975)], coppper, zinc, and gallium [Donaldson et al. (1976)], and various elements between iron and bromine [Gordon et al. (1980)]. More recently, Burkhalter et al. (1983) studied these L-shell transitions in chromium, manganese, iron, and nickel searching for line coincidences with H-like and He-like fluorine.

Bailey at al. (1986) measured the emission spectrum from a laser-irradiated microdot target consisting of a mixture of bromine, chlorine, sodium, and aluminum. The characteristic $\mathrm{K}$-shell emission of the aluminum and chlorine ions were used to diagnose the local plasma conditions which were also representative of the coexisting bromine ions present in the plasma. The space-resolved, time-integrated bromine L-shell $x$-ray emission spectrum was recorded for the same target shot. Seely et al. (1986), Goldstein et al. (19C7a), and Cerjan (1988) have presented detailed interpretations of this spectral data. Recently, Gauthier et al. 
(1985; 1986; 1987) measured the emission spectra from Ne-like Sr XXXX, Mo XXXIII, Rh XXXYI, and Ag XXXVII produced by a high-powered laser and noted the measurement of electric quadrupole radiative decays in these plasmas. In all of the above cases, the emission spectra were recorded using spatially-resolved, time-integrated instruments and therefore limiting the accuracy of the measured data due to unresolved temporal gradients.

In this chapter, I describe the X-ray spectroecopy experiments conducted on Mo $(Z=42)$ and Ag $(Z=47)$ microdot targets. These experiments were an extension of the $K$-shell spectroscopy experiments described earlier but applied to higher-Z targets and similar to the Bailey et al. (1986) experiments except with much better plasma diagnostics. These L-shell spectroscopy experiments employed the same simultaneously space and time resolved techniques to provide clear, precise spectral data to compare against available theoretical atomic models. Simultaneously space and time resolved L-shell $x$-ray spectra from these hot dense plasmas were recorded for the first time using the framing crystal X-ray spectrometer (FCXS), and also using the k-ray streaked crystal spectrograph with coarse spatial resolution (XSCS). Holographic interferometry provided independent and simultaneous measurements of the electron density profiles. The Mo targets were actually composite bull's-eye targets containing a mixture of $\mathrm{Mg}$ and $\mathrm{Mo}$. The $\mathrm{K}$-shell spectra emitted by the Mg ions allowed a rough estimate of the electron temperature of the plasma.

Several interesting features characteristic of the measured L-shell spectra are rexdily apparent in this work. First, the $\left.\left[2 s^{2} p^{6} 3 \mathrm{~d}(1 / 2,5 / 2)\right]=2\right]$ to $\left.\left[2 s^{2} 2 p^{6}(0,0)\right]=2\right]$, " $x$ " electric qudrupole iransition in Ne-like $\mathrm{Mo}^{32+}$ and $\mathrm{Ag}^{37+}$ ions have been identified in the recurded spectral data. I remind the reader that throughout this chapter (as in Chapter 4), I refer to the Ne-like levels using the electron configuration (dropping the $1 s^{2}$ term) along with the $\left(j_{1} j_{2}\right)$ and $j_{\text {lowl }}$ for each level. The related 
$\left.\left[2 s^{2} p^{6} 3 \mathrm{~d}(1 / 2,3 / 2)\right]=2\right]$ to $\left.\left[2 s^{2} 2 p^{6}(0,0)\right]=2\right]$, " $x 2^{\prime \prime}$ line is too weak to be observed. The measured intensity of the " $x$ " transition suggests population inversions between upper excited levels in Ne-like ions. The $(2 p-2 s)$ transitions, between the $2 s 2 p^{6} 3 d$ and $2 s^{2} 2 p^{5} 3 d$ level configurations, are interesting because they are less than $40 \AA$ in wavelength, and thus fall below the targeted "water window" required for biological applications of ahort wavelength lasers. The " $x$ " transition is predicted to be sensitive to variations in the electron density as discussed earlier in Chapter 4. I invectigate the effocts due to variations in the electron density on metastable Ne-like levels by comparing experimentally measured dipole allowed and electric quadrupole line intensitics to predicted theoretical line intensities.

In section 6.1, I describe the L-shell spectroscopy experiments on $\mathrm{Mo}$ and $\mathrm{Ag}$ microdot plasmas. An important parameter in characterizing these laser-produced plasmas is the eloctron temperature. In soction 6.2, the electron temperature based on Mg K-shell line intensity ratios are estimated for the Mo plasmas. In section 6.3, the L-shell line opacities have been cetimared for typical experimental plaema conditions. In section 6.4, the results of my experiments are presented including measurements of the density sensitive " $x / 3 A^{\prime}$ eloctric quadrupole to resonance line ratio. I will show that this line intensity natio is successfully modeled by quasi-steady atute collisional radiative theoretical models. I also examine a resonance line ratio " $3 \mathrm{~A} / 3 \mathrm{G}$ " in section 6.4.1 which is not modeled properly by current theory. Theae differences as well as speculations on poessible short wavelength laser candidates based on these motastable levels in Ne-like ions are discussed in section 6.4.2. Finally, I cammarize the experimental work on L-shell plasmus in section 6.5 . 


\subsection{Description of the L-Shell Experiments}

Two types of microdot torgets were used for these high-Z L-shell spectroscopy experiments. These microdot targets included a mixture of molybdenum and magnesium and the second target consisted entirely of silver. The $\mathrm{Mo} / \mathrm{Mg}$ microdot targets resembled square "bull's-eyes" and were mounted on thick (- $125 \mu \mathrm{m})$ mylar $\left(\mathrm{C}_{10} \mathrm{H}_{8} \mathrm{O}_{4}\right)$ substrates. A typical $\mathrm{Mo} / \mathrm{Mg}$ bull's-eye target was shown previously in Figure 3(left). The center square, with a side length of $70 \mu \mathrm{m}$, was composed of thin alternating layers of $\mathrm{Mo}(50 \AA \pm 10 \%)$ and $\mathrm{Mg}(400 \mathrm{~A} \pm 10 \%)$ with an overall composition of $1: 8$ in number density, respectively. This mixed dot was entered on a larger square, with a side length of $170 \mu \mathrm{m}$, composed entirely of $\mathrm{Mg}$. Ech dot was approximately $1.5 \mu \mathrm{m}$ thick. The Ag targets consisted of $25 \mu \mathrm{m}$ thicli $100 \mu \mathrm{m}$ diameter microdots of pure Ag mounted on thick $(>125 \mu \mathrm{m})$ polystyrene $(\mathrm{CH})_{x}$ substrates. Both types of targets were irradiated by a $526.0 \mathrm{~nm}, 1.0 \mathrm{~ns}$ wide stacked pulse using the CHROMA laser facility at KMS-Fusion. The Mo/Mg bull's-eye targets were heated to irradiances of $3.2 \times 10^{14} \mathrm{~W} / \mathrm{cm}^{2}$. The Ag targets were heated to irradiances of $4.1 \times 10^{14} \mathrm{~W} / \mathrm{cm}^{2}$. The CHROMA heating laser beam was focused (far field) onto the planar target surface to a diameter of $250 \mu \mathrm{m}$ using an $f / 2.5$ lens, thus overfilling the microdot target. As the laser heats the surface, material from the target biows-off away from the target surface and is confined radially by the expanding colum of low $\mathbf{Z}$ (substrate) material surrounding it. This creates an excellent, nearly one-dimensional spectroscopic source as described in Chapter 2.

A wide array of plasmi diagnostics were fielded together to study the emission from the laser-produced plasmas. The instruments were aligned to view the target nearly perpendiculiar to the laser axis as shown before in Figure 7. A common timing fiducial provided time sccuracies of 5 ps for all of the time resolved 
instruments. All times quoted represent the elapsed time from the start of the CHROMA heating pulse ( $\mathrm{T}=0$ is the peak of the first of the ten $100 \mathrm{ps}$ wide stacked pulses). The X-ray streaked crystal spectrometer (XSCS) was also used to record the temporal characteristics of the emission. Because of its coarse spatial resolution and the uncertainty as to its precise field of view, the data recorded with this instrument could not be fully interpreted.

The Framing Crystal X-ray Spectrometer (FCXS) provided the simultaneously space and time resolved measurement of $\mathrm{Mo}$ and $\mathrm{Ag} \mathrm{L-shell} \mathrm{emission} \mathrm{spectra.} \mathrm{The}$ FCXS is fully described in Chapter 2. For these experiments, the FCXS was operated using $250 \mathrm{ps}$ wide (actual gate) time frames and $50 \mu \mathrm{m}$ wide imaging slits with a magnification of 8.5 providing approximately $55 \mu \mathrm{m}$ spatial resolution. The FCXS was operated in several different modes for these experiments. For the Mo/Mg experiments, two different x-ray diffraction crystals were used: the Mo L-shell spectra was measured over a wavelength range of 3.5 to $5.4 \AA$ with a spectral resolution, $(\lambda / \Delta \lambda)$, of approximately 1000 using a beryl crystal $(2 \mathrm{~d}=15.96 \mathrm{~A})$; the $\mathrm{Mg} \mathrm{K}$-shell spectra was measured over a wavelength range of 5.5 to $8.5 \mathrm{~A}$ with a spectral resolution of about 700 using a KAP crystal $(2 \mathrm{~d}=26.63 \mathrm{~h})$. Two of the three time frames recorded the Mo L-shell spectra. The third FCXS time frame recorded the $\mathrm{Mg} \mathrm{K}$-shell emission corresponding to the exact time interval as one of the Mo L-shell time frames.

A set of framed Mo L-shell spectra for this experiment is shown in Figure 52. [Shot $\# 8502$ at $3.2 \times 10^{14} \mathrm{~W} / \mathrm{cm}^{2}$ ] Each strip in this figure represents the recorded spectra versus the spatial extent of the plasma emission (vertically) over a $250 \mathrm{ps}$ time interval. The Mo L-shell spectra was measured at 230-480 ps (middle strip) and 730-980 ps (top strip). The bottom strip shows $\mathrm{Mg}$ K-shell spectra measured at 230-480 ps. In Figure. 53 are sets of line outs of the recorded Mo L-shell and Mg 
Figure 52. FCXS data for Shot \#8502 (1:8 Mo:Mg bull's-eye target shot at $3.2 \mathrm{x}$ $10^{14} \mathrm{~W} / \mathrm{cm}^{2}$ ) showing the spatially resolved Mo L-shell radiation emitted during the time intervals $230-480 \mathrm{ps}$ (middle) and $730-980 \mathrm{ps}$ (top), and the $\mathrm{Mg} \mathrm{K}$-shell radiation $230-480$ ps (bottom). Note: the plasma image appears to be rotated relative to the FCXS striplines. This is due to a slight misalignment (rotation) of the imaging slits and does not alter the spatial imaging or temporal information measured for this shot. 


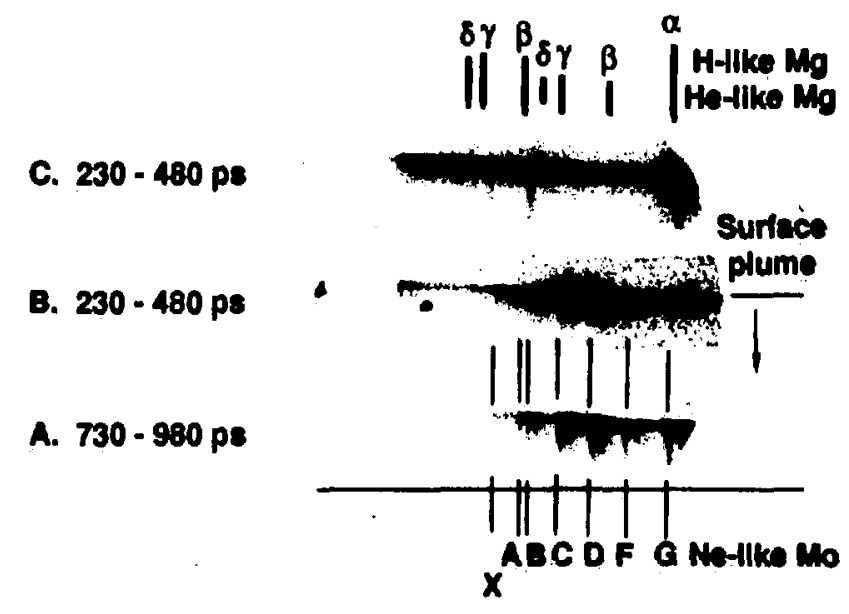

Figure 52 
Figure 53. Densitometer scans showing the Mo L-shell spectra (Shot \#8502) emitted from $50 \mathrm{\mu m}$ wide regions of plasma at various distances from the target surface for the time intervals a) $230-480 \mathrm{ps}$, b) $730-980 \mathrm{ps}$, and the $\mathrm{Mg} \mathrm{K}$-shell spectra for c) $230-480 \mathrm{ps}$. 


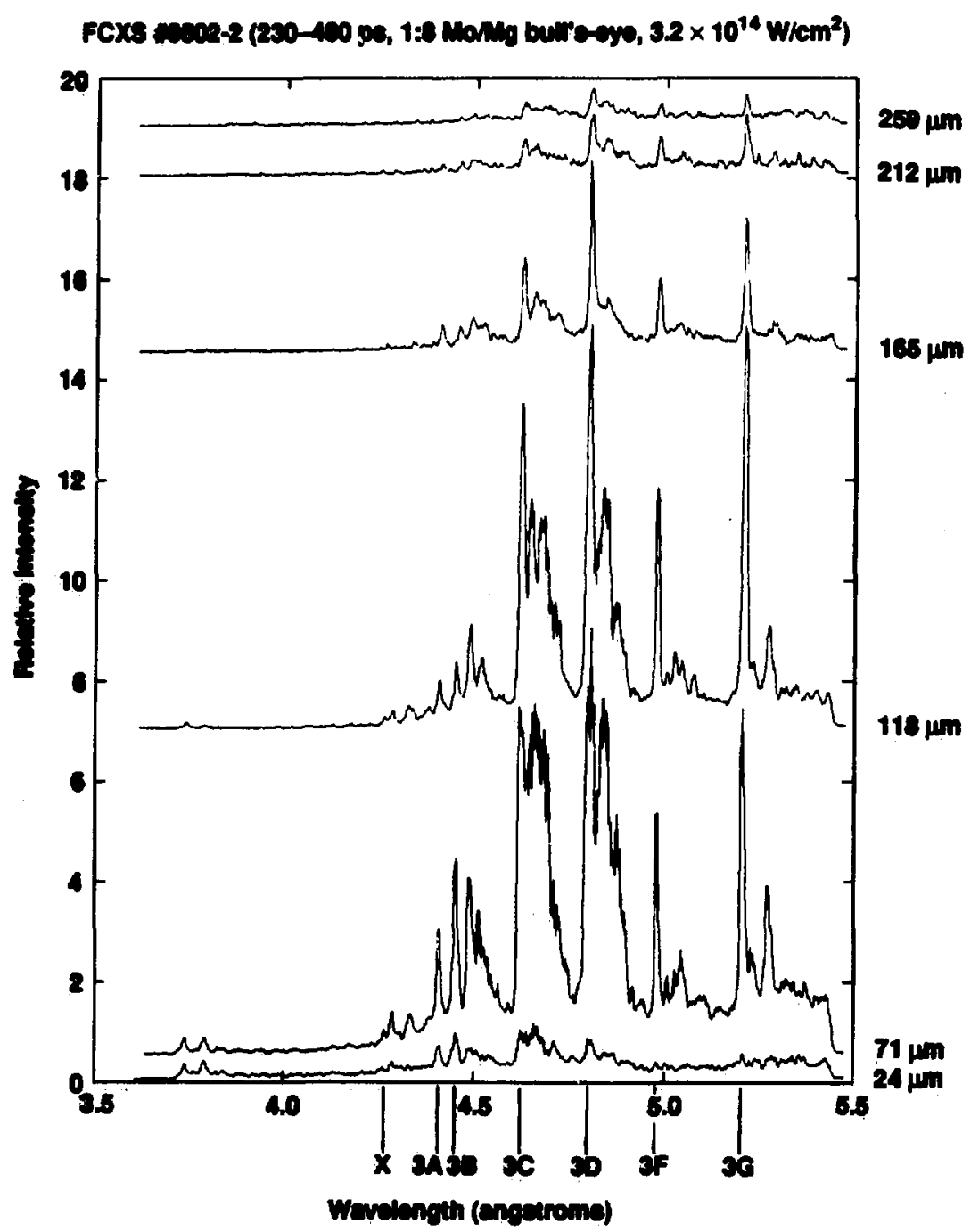

Figure 53a 


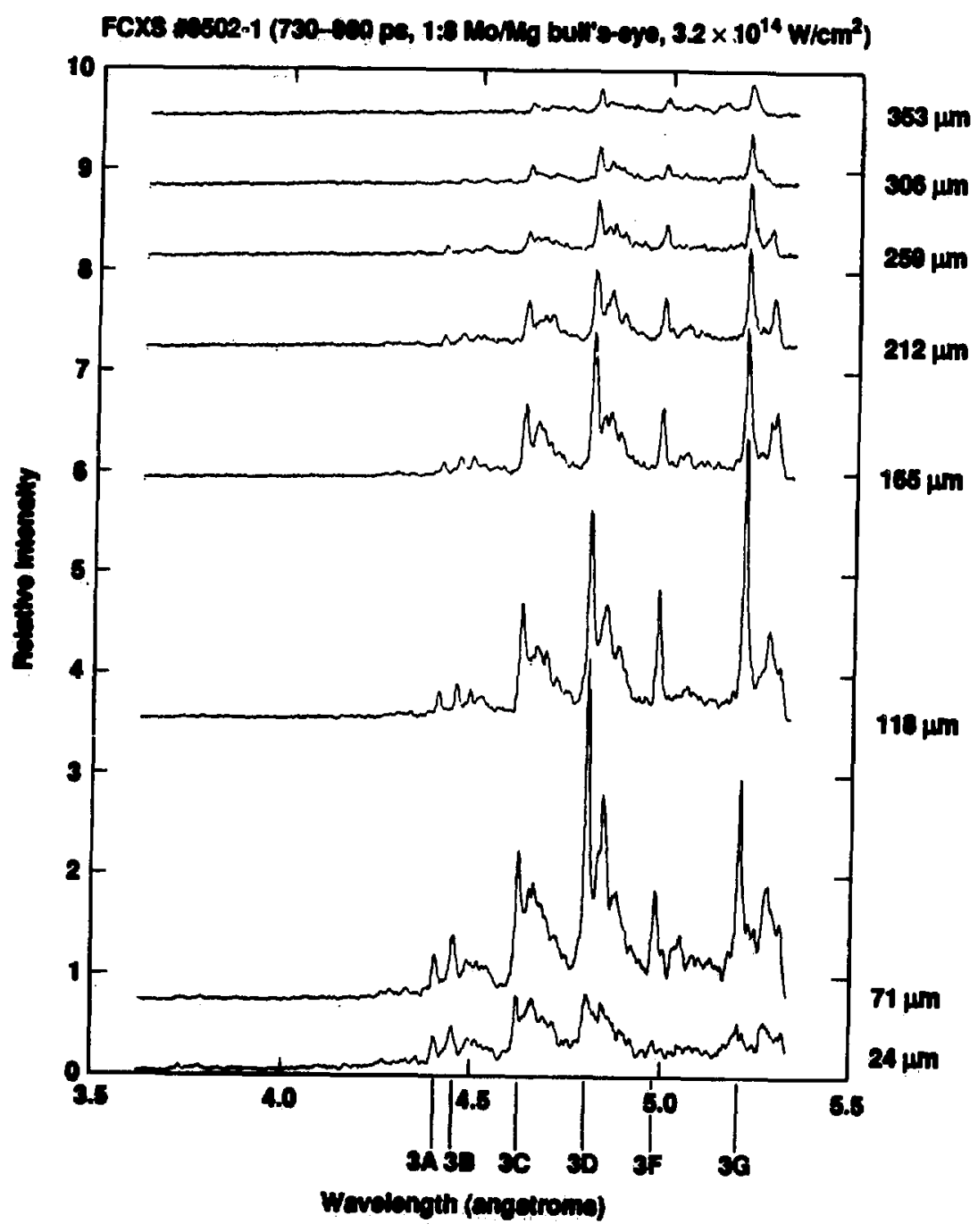

Figure 53b 


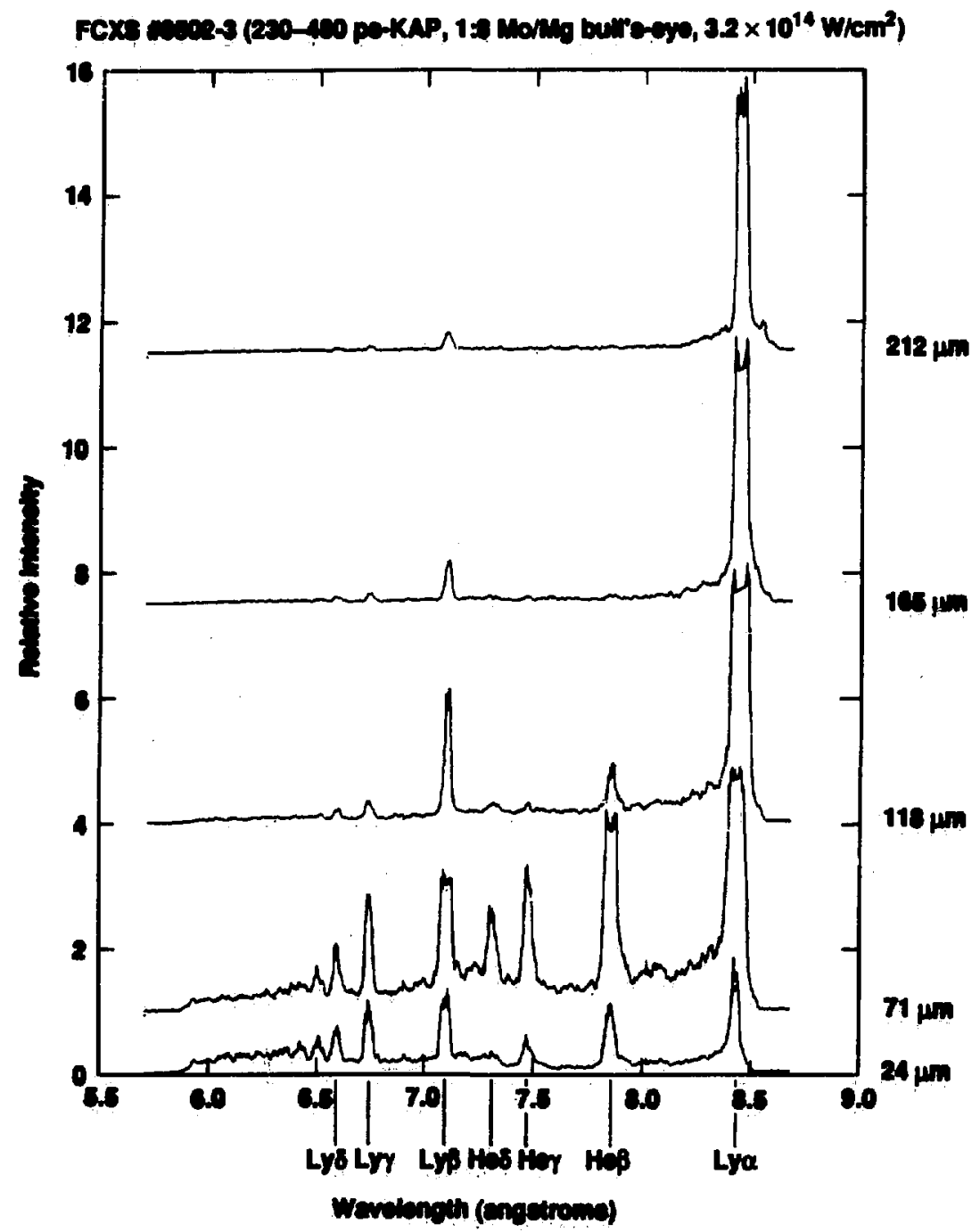

Figure 53c 
K-shell spectra produced by $400 \mu \mathrm{m}$ (film) wide densitometer scans of the FCXS data. [Shot \#8502] These line outs represent relative spectral line intensities emitted during the FCXS frame time interval from $50 \mu \mathrm{m}$ regions of plasma centered at various distances from the target surface. These densitometer scans show the large spatial and temporal variations in these Ne-like resonance lines as well as the blended satellite lines (Na-like and Mg-like) which appear on the long wavelength side of the resonance lines. These dramatic spatial and temporal variations in the Ne-like resonance lines are observed for the first time due to the new simultaneously space and time resolved instruments. The electric quadrupole transitions are also identified in the earliest time frame with the " $x$ " line at approximately $4.27 \AA$ and the $3 p-2 p$ lines (labeled "y1", "y2", and "y3") possibly seen between 4.8 and $5.1 \AA$. The 3d-2s " $\times 2$ " line is usually too weak to be observed. The electric dipole allowed and quadrupole $\mathrm{x}-\mathrm{my}$ transitions for $\mathrm{Ne}-$ like $\mathrm{Mo}^{32+}$ and $\mathrm{Ag}^{37+}$ are listed in Table 4 shown earlier in Chapter 4.

In addition, a second Mo/Mg bull's-eye target shot has been analyzed and some of the data from this shot is presented here. This second Mo/Mg target [Shot \#8504] was heated to $3.0 \times 10^{14} \mathrm{~W} / \mathrm{cm}^{2}$ and all three FCXS frames sampled the same $730-980$ ps time interval. The two striplines utilizing the beryl crystal, were operated at two different MCP bias voltages providing a wider dynamic range in measuring the Mo L-shell spectra. In fact, with the gain increased by approximately $3.5 X$ (generated by increasing the MCP bias voltage by approximately 100 volts), the weak " $x$ " electric quadrupole line was observed. The third stripline measured the Mg K-shell emission diffracted off of the KAP crystal during the same time period. For all shots, line intensities from the same FCXS time frame were used to measure the " $x / 3 A$ " or " $3 A / 3 G "$ line ratios.

All intensities have been corrected for the film response, crystal reflectivities, filter 
transmissions, and estimated photocathode efficiencies [Burek (1976); Rocket et al. (19985; 1986); Glinsky (1985); Henke et al. (1981; 1982; 1986); and Fraser et al. (1984)]. The Mo Ne-like resonance lines were identified by comparing the observed wavelengths and intensity patterns with those calculated using Scofield's relativistic multiconfigurntionel Dirac-Fock "RAC" code. These simultaneous space and time resolved measurements were not highly spectrally resolved and did not allow precise, independent experimental wavelength measurements. The large number of unresolved satellite lines also prevent clear and detailed indentification of all the spectral features. The instrumental response should not be a problem for the " $x / 3 \mathrm{~A}^{\text {" }}$ line ratio as the lines are nearly adjacent to each other spectrally. The "3A/3G" line intensity will be more censitive to uncertainties in the instrumental response. This points out that calibrated instrumentation is essential for future studies.

For the Ag microdot experiments, a flat ADP ( $2 \mathrm{~d}=10.648 \AA)$ diffraction crystal was used to measure the $\mathrm{Ag} \mathrm{L}$-shell emission spectra over a wavelength range of 2.7 to $4.4 \mathrm{~A}$ with a spectral resolution ff over 1000 . The three FCXS time frames recorded the Ag L-shell spectra over a sitquence of sample times. A set of framed Ag L-shell spectra for this experiment is shown in Figure 54. The three strips correspond to the recorded apectra versus the spatisl extent of the plasma emission measured at $230-480 \mathrm{ps}$ (middle strip), 560-810 ps (bottom strip), and 840-1090 ps (top strip). In Figure 55 are sets densitometer scans of this same Ag shot. The Ne-like Ag resonance lines were identified using theoretical wavelengths provided by Scofield's "RAC" code. Again, groups of unresolved satellite lines appear as blended lines. For the experiments reported here, the $\mathrm{Mo}$ and $\mathrm{Ag} \mathrm{L-shell} \mathrm{spectra} \mathrm{is}$ not fully analyzed. A complete and detailed analysis of the Mo and Ag L-shell spectra is currently in progress and will be presented elsewhere.

The 4-Frame Holographic Interferometer was operated simultaneously with the FCXS thus providing an independent, non-spectroscopic measurement of the spatial 
Figure 54. FCXS data for Shot $\$ P 519(100 \mu \mathrm{m} \mathrm{Ag} \mathrm{microdot} \mathrm{target} \mathrm{shot} \mathrm{at} 4.1 \times$ $10^{14} \mathrm{~W} / \mathrm{cm}^{2}$ ) showing the spatially resolved $\mathrm{Ag}$ L-shell radiation emitied during the time intervals $230-480 \mathrm{ps}$ (middle), 840-1090 ps (top), and 560-810 ps (bottom). 


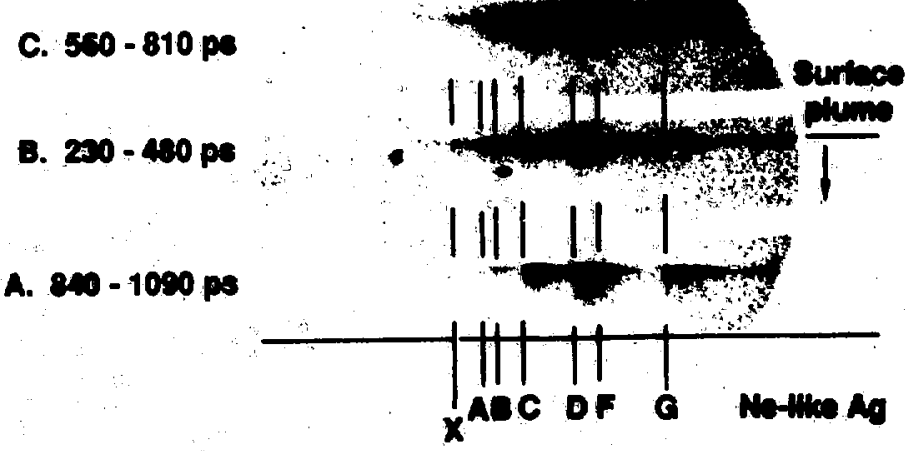

FIgure 54 
Figure 55. Densitometer scans showing the Ag L-shell spectra (Shot \#8519) emitted from $50 \mu \mathrm{m}$ wide regions of plasma at various distances from the target surface for the time intervals a) $230-480$ ps, b) $560-810$ ps, and c) $840-1090$ ps. 


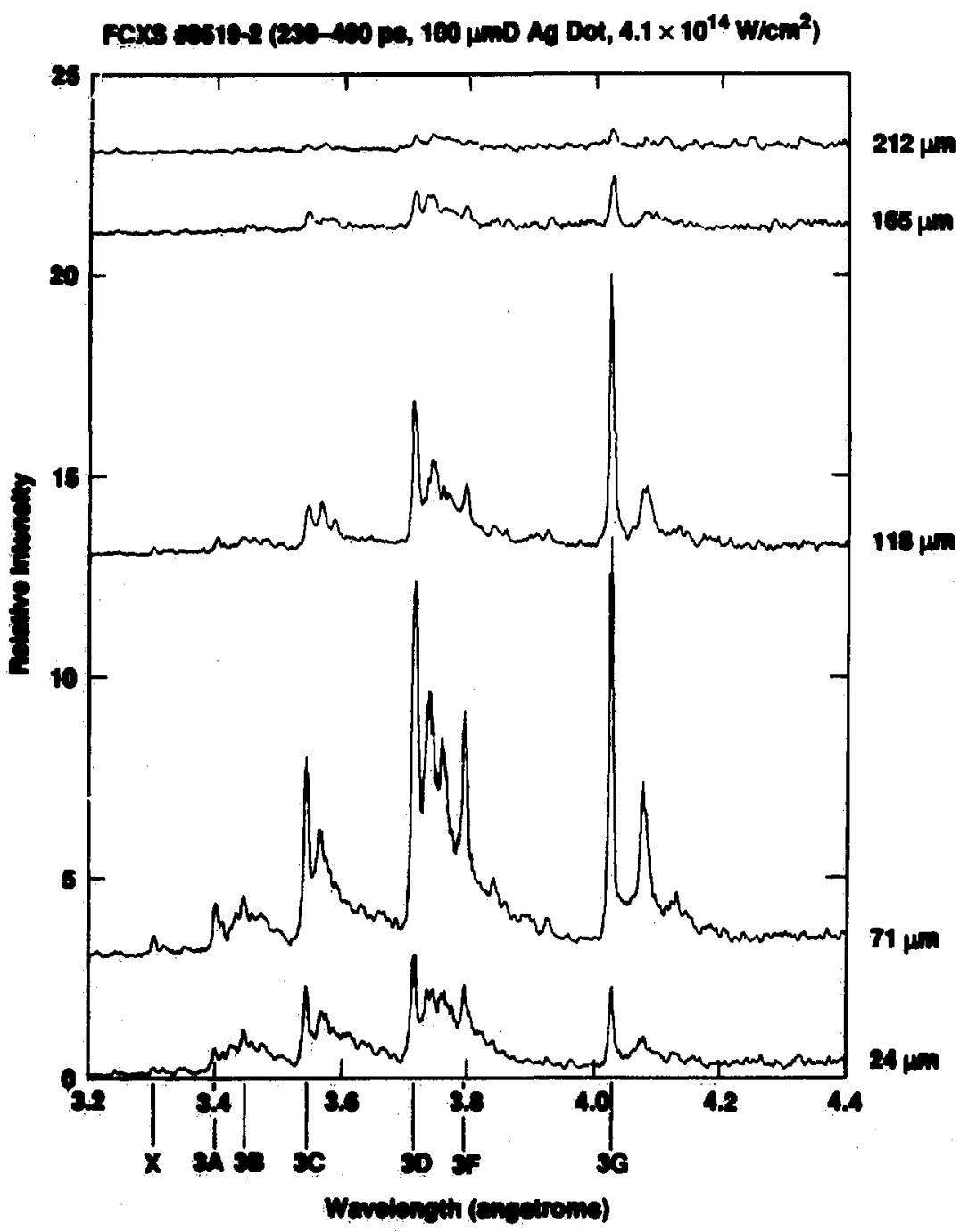

FIgure $55 a$ 


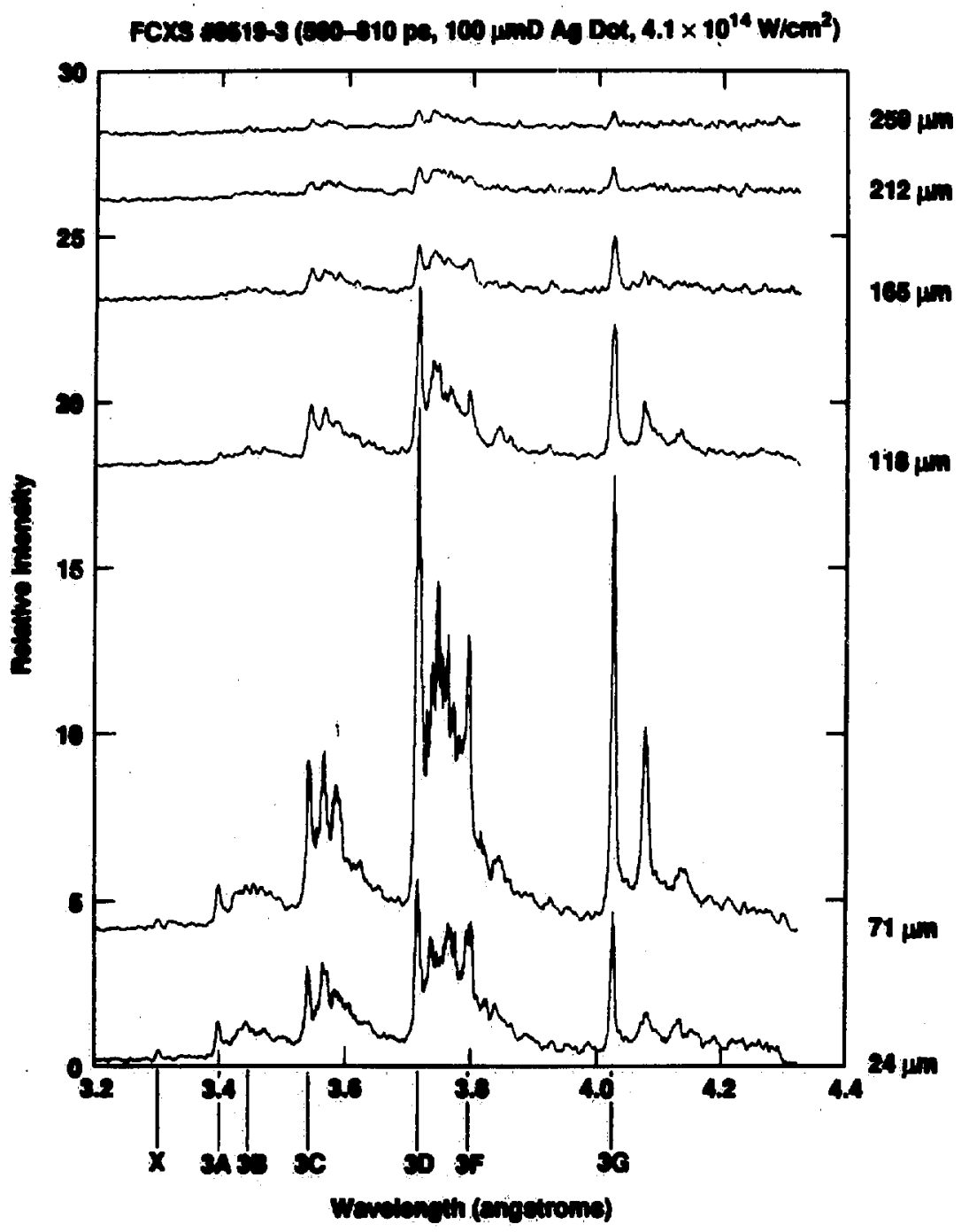

Figure 55b 


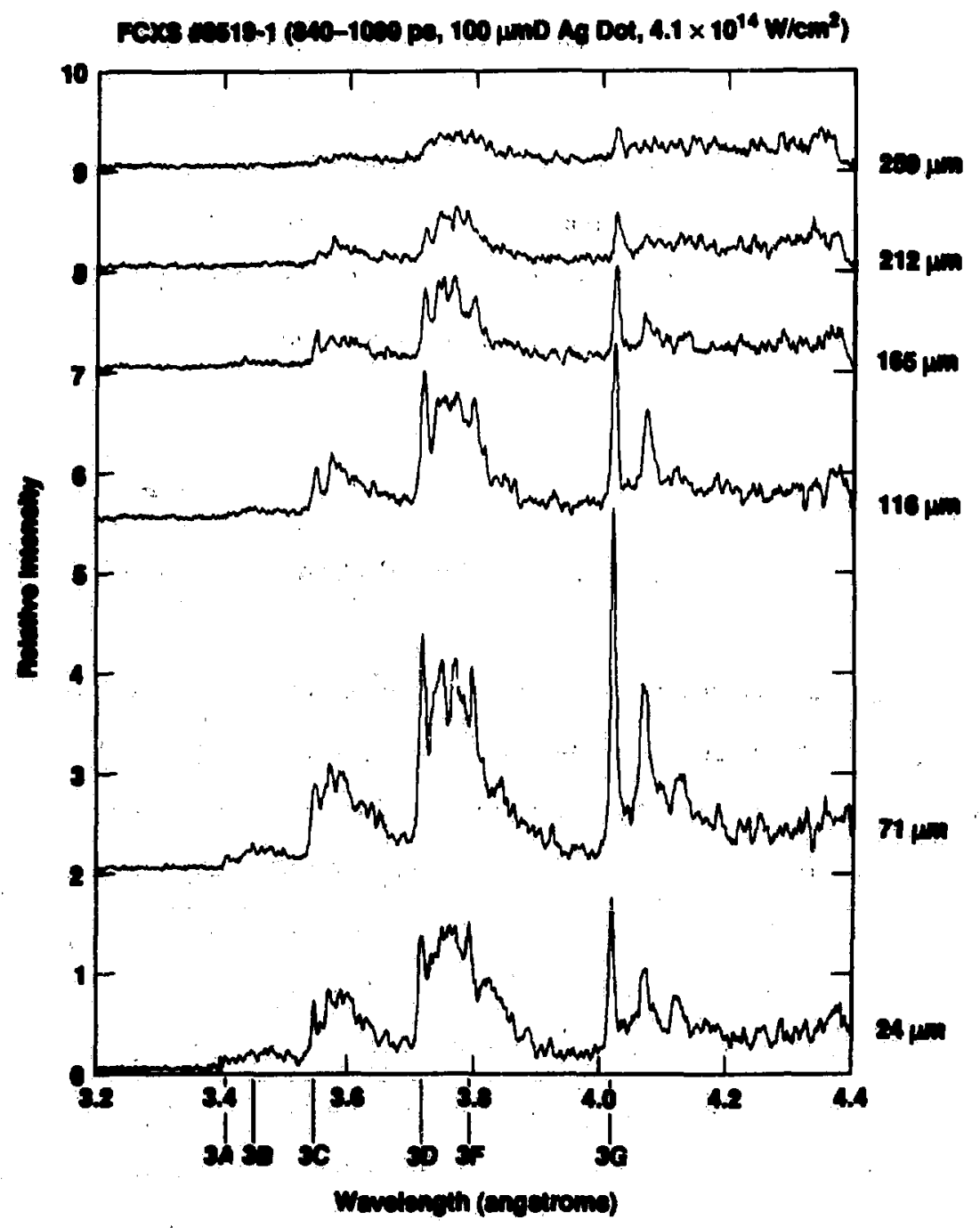

Figure 55c 
electron density profiles for four different probe times separated by $200 \mathrm{ps}$. These density profiles for the $\mathrm{Mo} / \mathrm{Mg}$ shot [ $\$ 8502$ ] (beginning at $357 \mathrm{ps)}$ and the $\mathrm{Ag}$ microdot shot [*8519] (beginning at 457 ps) are presented in Figures 56(top) and 56(bottom), respectively. The signal-to-noise level was very poor for the first interferometry time frame [Shot *8519:257 ps] and was not useable. Based on the hydrodynamic simulations of $\mathrm{K}$-shell targets presented in Chapter 5 , the interferometric data falls off exponentially (well fit by strnight lines on a $\log$ plot) and can be extrapolated to repions close to the target surface. This approximation should be valid for regions of plasm beyond the critical density, 20-50 $\mathrm{m}$ from the target surface. [See Chapter 3.]

\subsection{Extimates of the Electron Temperature}

The haser-produced L-shell plasma must be fully characterized in onder to properly investigate the density sensitivity of specific spectral emission lines. As described earlier in this thesis, the electron density profiles were measured using holographic interferometry. This provided a clear, model independent determination of the densities in the laser-plasm. An independent measurement of the electron temperature was also required for these experiments. For the Mo/Mg bull's-eye shots, the initial plan was to measure the electron temperature using the slope of the free-bound recombination continuum of $\mathrm{H}$-like $\mathrm{Mg}$ ions. Unfortunately, the $2^{\text {nd }}$ order integrated reflectivity for the KAP diffraction cryatal is quite high and although the $\mathrm{H}$-like $\mathrm{Mg}$ free-hound continuum is measured, it is contuminated by coincident 2 ad onder Mo $(n \geq 4)$ to $(n=2)$ spectral lines. Instend, the electron temperature was estimated using the $\mathrm{Mg} \mathrm{He}$-like to $\mathrm{H}$-like resonance line ratio. Experimental line 
Figure 56. Holographic interferometry electron density (axial) profiles at various probe times for (top) Shot $\# 8502, \alpha \mathrm{Mo} / \mathrm{Mg}$ bull's-eye plesma at $3.2 \times 10^{14} \mathrm{~W} / \mathrm{cm}^{2}$ and (bottom) Shot $\# 8519$, a Ag dot plasma at $4.1 \times 10^{14} \mathrm{~W} / \mathrm{cm}^{2}$. 

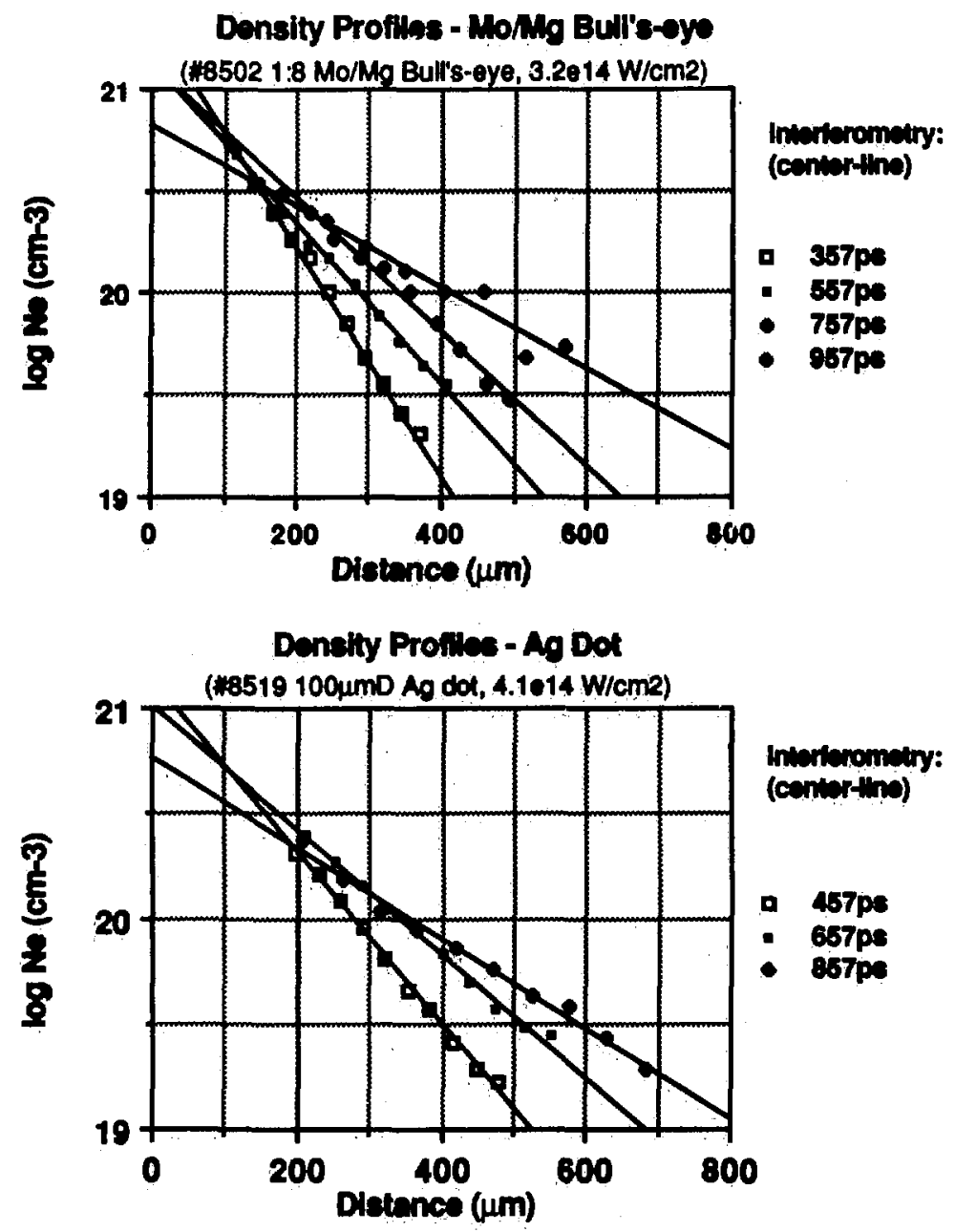

Figure 56 
intensity ratios of He-like to $\mathrm{H}$-like resonance lines were compared with those predicted by the "RATION" (CRE) model [Lee et al. (1985); Cauble (1987)] to infer the electron temperature profile. Although this steady state (CRE) ionization analysis is not appropriate for this transient plasma, it gives some qualitative information on the electron temperature profile. The temperature profile estimated using this analysis tends to increase slightly away from the target surface; about $350 \mathrm{eV}$ at 24 $\mu \mathrm{m}$ and near $850 \mathrm{eV}$ at $212 \mu \mathrm{m}$ from the target surface for this time interval. [See Figure 57.] These temperatures were estimated using both the He-like $1 \mathrm{~s}^{2}\left({ }^{1} \mathrm{~S}_{0}\right)-$ 1s3p $\left({ }^{1} P_{1}\right)$ "Hey" to H-like $1 \mathrm{~s}-3 \mathrm{p}$ "Ly ${ }_{\gamma}$ " resonence line ratio and the He-like $1 \mathrm{~s}^{2}\left({ }^{1} \mathrm{~S}_{0}\right)-1 \mathrm{~s} 4 \mathrm{p}\left({ }^{1} \mathrm{P}_{1}\right)$ "Heg" to H-like $1 \mathrm{~s}-4 \mathrm{p}$ "Lys" resonance line ratio and predict similar temperature profiles for both the $230-480 \mathrm{ps}$ and $730-980 \mathrm{ps}$ time frames. [Shots $\$ 8502\left(3.2 \times 10^{14} \mathrm{~W} / \mathrm{cm}^{2}\right)$ and $\$ 8504\left(3.0 \times 10^{14} \mathrm{~W} / \mathrm{cm}^{2}\right)$, respectively] These temperature values are similar to the temperature profile for the Al microdiot plasmas described in the previous chapter. Although the $\mathrm{Al}$ targets were irradiated by a lower laser irradiance $\left(1.0 \times 10^{14} \mathrm{~W} / \mathrm{cm}^{2}\right)$, the temperature profile determined from the slope of the H-like free-bound recombination continuum ranges from approximately $200 \mathrm{eV}$ near the surfece to $500 \mathrm{eV}$ at $200 \mu \mathrm{m}$ out in the plasm plume during the laser heating pulse. Additionally, the aluminum microdot experiments indicabed that the CRE He-like to H-like resonance line ratio predicts temperatures consistently higher than the free-bound recombination temperature during the heating laser pulse. The resonance line to dielectronic satellite line intensity ratio would have provided a better estimate for the true electron temperature but I was unable to measure these spectral lines due to the limited spectral coverage of the FCXS instrument used in the L-shell experiments. [See Chapter 5.] For these experiments, an "average" temperature of $500 \mathrm{eV}$ was used to analyzed the $\mathrm{Mo}^{32+}$ and $\mathrm{Ag}^{37+}$ spectra in the following section. It will be shown that the density sensitive Ne-like 
Figure 57. The electron temperature is estimated using the "He/Ly" and the "Hes/Ly8" resonance line ratio based on the "RATION" (CRE) model for (top) 230-480 ps (Shot *8502) and (bottom) 730-980 ps (Shot *8504) for the Mo/Mg bull's-eye plasmus. 
Electron Temparahere vis CRE Reconanco Line Ratio
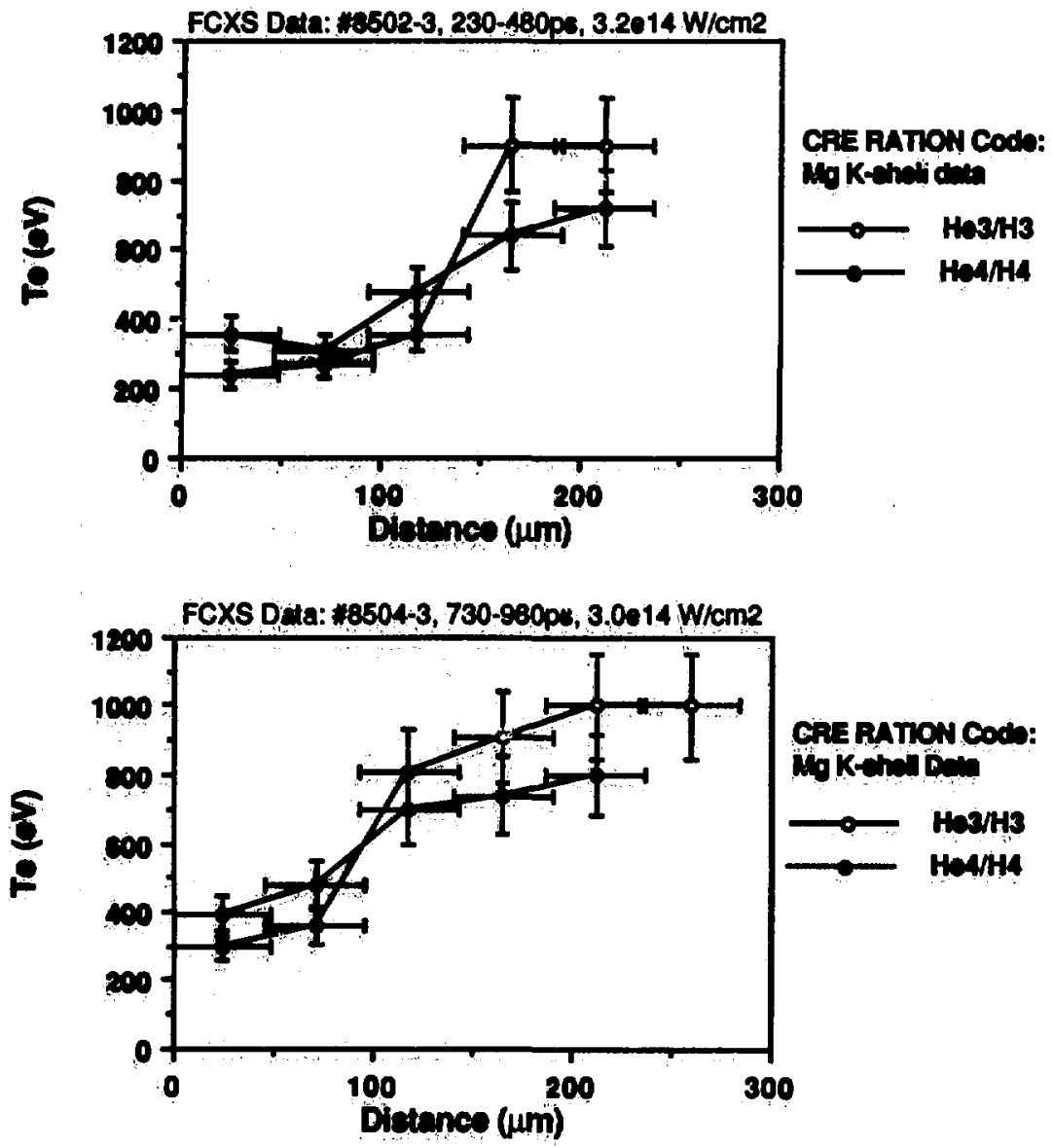

FIgure 57 
spectral emission features studied in this work (especially the " $x / 3 A$ " line ratio) are only slightly temperature dependent.

\subsection{Extimates of the Line Opacity of the L-Shell Plasmas}

We can make a quick estimate of the target opacity using the same formula presented in Chapter 3 for $\mathrm{K}$-shell resonance lines. We find that for the plasma conditions typical for these experiments, both the Mo/Mg bull's-eye and pure Ag microdot plasmas were optically thin (at most only a few optical depths thick for the strongeat resonance Ne-like line). In Table 8, I show the estimated center-line opacities computed for some of the Ne-like resonance lines in both $\mathrm{Mo}$ and $\mathrm{Ag}$ for the plasma conditions typical for these experiments where I have assumed an electron density of $10^{21} \mathrm{~cm}^{-3}$, a temperature of $500 \mathrm{eV}$, and an estimated Ne-like ground state fraction of 30\%. This is only a very crude estimate of the target opacity. I remind the reader that the line opacity (optical depth) ecales roughly linearly with the charge state fraction, the electron (ion) density, and as $T_{i}{ }^{1 / 2}$.

If the plasma were to be optically thick, then the $\left.\left[2,22 p^{5} 3 \mathrm{~d}(3 / 2,5 / 2)\right]=1\right]$ excited level of the strongest "3D" resonance emission line would be expected to be trapped. As shown earlier in Table 6, the " $x$ " excited level is collisionally coupled to the 2p3d configuration. From Table 5a (Chapter 4), the collisional (\$) flux feeding the " $\mathrm{x}$ " excited level from the "3D" excited state level is of onder $0.1 \% \mathrm{at} 10^{21} \mathrm{~cm}^{-3}$. Therefore, opacity effects should be minimal on the" $x$ " level population. The "3A" level is not directly coupled to the "3D" level. Therefore, this very simple analysis would indicate that opacity effects should not adversely affect the " $x / 3 \mathrm{~A}$ " measurement. However, if the plasma was optically thick, there would be an increased excited state population which would redistribute itself via collisional 
Table 8. Simple estimates of the line opecities for several $L$-shell emission lines are listed for $T_{e}=T_{j}=50 \mathrm{CV}, \mathrm{n}_{\mathrm{e}}=10^{21} \mathrm{~cm}^{-3}$, Ne-like charge state population of $f_{(\text {No-like) }}=30 \%$, and based on the radius of the target as the nominal plasma size. 


\section{L-Shell Target Line Opacity Estimates}

Tune Type: $\quad$ 1:8 Mo/Mg Bull's-eye.

$70 \mu \mathrm{m} / 170 \mu \mathrm{m}$ squares; thick mylur substrate.

Teraet Show: $\$ 8502,8504$

Don. Number. Enction: [reblave to the electron density, ne $\left.\left(\mathrm{cm}^{-3}\right)\right]$
For the center dot (equare), there are $8 \mathrm{Mg}$ ions for ewch Mo ion. The Mg ions are ascumed to be +11 tifies ionized (H-like); the Mo ions are aceunad to be +32 timies ionizod (Ne-like).

(No. of Mo ions) $)_{e}=1 / 120$

\begin{tabular}{|c|c|c|c|}
\hline Nonjike linas: & $\lambda(\AA)$ & $A_{i j}\left(\sec ^{-1}\right)$ & $\tau_{0}^{*}$ \\
\hline $\boldsymbol{\lambda}$ & 4.273 & $3.0 \times 10^{11}$ & 0.020 \\
\hline$" 3 A "$ & 4.415 & $3.5 \times 10^{13}$ & 1.110 \\
\hline "3D" & 4.804 & $1.8 \times 10^{14}$ & 7.440 \\
\hline $3 G^{n}$ & 5.208 & $1.1 \times 10^{13}$ & 0.570 \\
\hline
\end{tabular}

$100 \%$ f g Microdot

$100 \mu$ m diameter, thick $\left(\mathrm{CH}_{n}\right.$ substrate.

\#8515, 8517, 8519

The Ag ions are assumed to be +47 times icnized (Ne-like).

(No. of $A g$ ions) $/ n_{e} \approx 1 / 47$

\begin{tabular}{l|l|c}
$\lambda(\AA)$ & $\mathrm{A}_{\mathrm{ij}}\left(\sec ^{-1}\right)$ & $\tau_{0}^{*}$ \\
\hline 3.299 & $6.2 \times 10^{11}$ & 0.053 \\
3.399 & $5.9 \times 10^{13}$ & 2.340 \\
3.718 & $3.3 \times 10^{14}$ & 17.12 \\
4.019 & $1.8 \times 10^{13}$ & 1.179
\end{tabular}

*Line opacity estimates are based on $\mathrm{T}_{\mathrm{e}}=\mathrm{T}_{\mathrm{i}}=500 \mathrm{eV}, \mathrm{n}_{\mathrm{e}}=10^{21} \mathrm{~cm}^{-3}$, and $f_{(\mathrm{Ne}-\mathrm{Hi} e}=30 \%$. 
mixing and radiative cascades. This could indirectly affect the "x/3A" line ratio and potentially "mask" the density dependence examined in this work. Therefore, detnilod calculations should be undertaken to address this issue properly. Despite the Lrge variations in the plasma conditions (temperature, electron density, and ion fraction), the L-shell microdot plasmins studied in the experiments reported here were generally optically thin.

\subsection{The " $x$ " Diectric Quadrupole to "3A" Rewonance Line Ratio: Reculte and Dincencion}

In this section, I will focus on one particular electric quadrupole transition, $\left.\left[2 s^{2} p^{6} 3 d(1 / 2,5 / 2)\right]=2\right]$ to tive $\left.\left[2 s^{2} 2 p^{6}(0,0)\right]=0\right]$ ground state. This electric quadrupole tranaition " $\mathrm{x}$ " has been observed in time-integrated spectra from laser-paduced plasmas by Gauthier et al. (1985; 1986; 1987) for a range of element from strontium $(Z=38)$ is silver $(Z-47)$ although an analysis of this data has not yex been published. This transition has also been observed in rokamak planem by Beiendofer et al. (1987) and Rice et al. (1988) where it is labeled as "E25". The clectric quadrupole transition " $x$ " is observed in both the Mo and Ag target shous presented here appearing epectrally to the short wavelength side of all Ne-1lse $(n=3)-(n=2)$ reconance line transitions, fairly isolated from other spectrocopic features. The electric dipole transition, "3A" $\left[2 s 2 p^{5} 3 p(1 / 2,3 / 2) J=1\right]$ to the $\left.\left[2 s^{2} 2 p^{6}(0,0)\right]=0\right]$ ground state occurs spectrally nearby so that both the " $x "$ and "3A" lives are clearly mensured for the Mo and Ag target shots and definively show veriations in intensity as a function of distance out into the plasma plume. Since these limes occur epectrally nearby to each other, uncertainty in the instrumental 
response was not a problem. Both lines appear strongest in relative intensity at about $50 \mu \mathrm{m}$ from the target surface and disappearing (very low signal to noise level) at about $250 \mu \mathrm{m}$ during the $230-480$ ps time frames [Shots $* 8502$ and $* 8519$ ]. They are clearly shown in magnified blow ups of digitized scans shown in Figure 58 for Mo and Ag, respectively [Shots $\$ 8502$ and $\$ 8519$ ]. In both cases, the data represents the L-shell spectra emitted from a region of plasma 47 to $94 \mu \mathrm{m}$ from the target surface for the time interval of $230-480 \mathrm{ps}$. The expanded section of spectra represents a $5 X$ magnification of the relative spectral line intensity.

In Figure 59, the calculated $\mathrm{Mo}^{35+}$ and $\mathrm{Ag}^{37+}$ " $\times / \mathrm{BA}^{4}$ line ratios are plotted as a function of the electron density for a temperature $500 \mathrm{eV}$ derived from the 37 level "NELI" and the steady state, 89 level Ne-like collisional radiative models. The experimental " $\times / 3 A^{n}$ line ratios are also plotted in Figure 59 based on the corresponding incerferometric densities (open circles). The agreement between experiment and theory is excellent over the density range of $1.0 \times 10^{20}$ to $1.0 \times 10^{21}$ $\mathrm{cm}^{-3}$ covered in these experiments.

This comparison between the theoretically predicted and experimentally measured " $x / 3 A^{\prime}$ line ratios can also be illustrated by using the model to derive the electron density profile based on the measured line intensity ratios. This is similar to the analysis uned to determine the He-like resonance to intercombination density profiles presented in Chapter 5. This method is useful for ratios which are sensitive to the electron density due to collisions among excited states and less sensitive to variations in the electron temperature or the ionization distribution. The electron densities

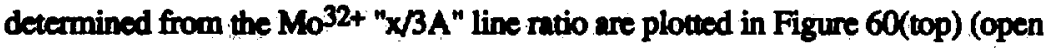
circles) for $230-480 \mathrm{ps}$ assuming a $500 \mathrm{eV}$ temperature [Shot $\$ 8502$ at $3.2 \times 10^{14}$ W/cm²]. Similarly, electron densities determined from the $730-980 \mathrm{ps} \mathrm{Mo}^{32+}$ " $x / 3 A^{n}$ line ratio are shown in Figure 60(bottom) [Shot $\# 8504$ at $3.0 \times 10^{14} \mathrm{~W} / \mathrm{cm}^{2}$ ] 
Figure 58. FCXS spectra representing a plasma region $47-94 \mu \mathrm{m}$ from the target surface for 230-480 ps for the a) Mo and b) Ag plasma. The short wavelength region has been magnified by a factor of $5 \mathrm{X}$ to show the weak electric quadrupole line, " $x$ ". 

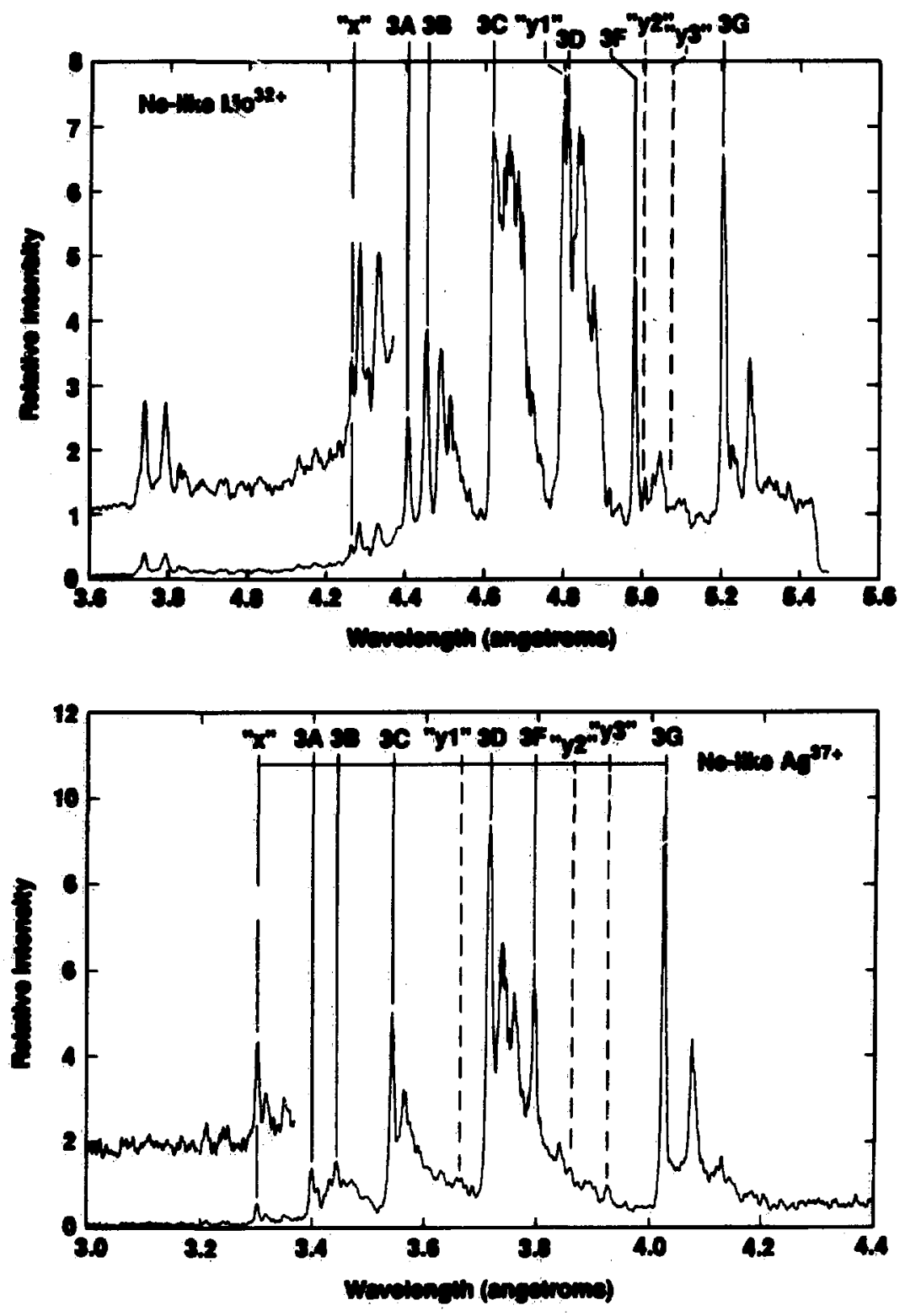

Figure 58 
Figure 59. A comparison of experimentally measured and theoretically predicted density dependence of the " $\times / 3 \mathrm{~A}$ " line ratio for Ne-like $\mathrm{Mo}^{32+}$ (top, Shot $\# 8502$ ) and $\mathrm{Ag}^{37+}$ (bottom, Shot "8519). Both the 37 level "NELI" and 89 level Ne-like models have been used assuming $T_{e}=500 \mathrm{eV}$. 

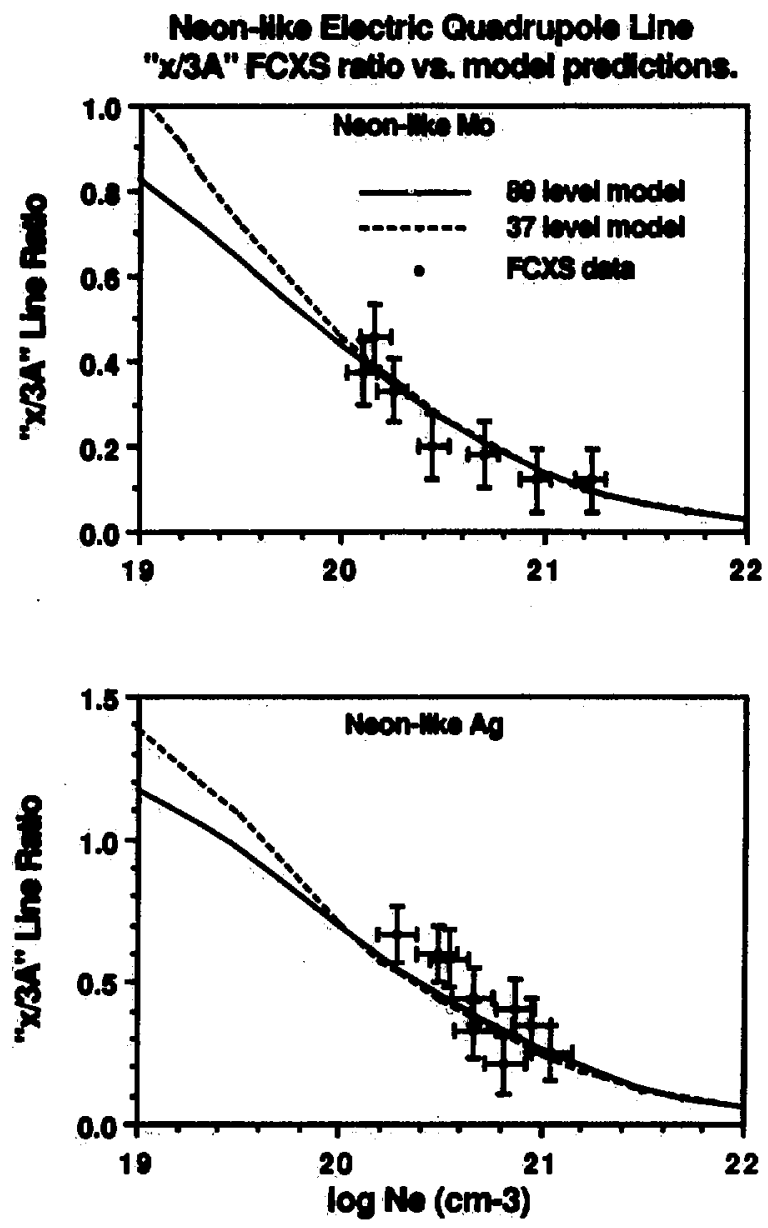

Figure 59 
Figure 60 . Density profiles using the Ne-like Mo " $x / 3 A$ " line intensity ratio agree with interferometry profiles for (top) 230-480 ps (Sirot $\# 8502$ ), and (top) 730-980 ps (Shot "8504). The 37 level "NELI" model has been used in each case based on $T_{e}=500 \mathrm{eV}$. 
Exctron Denetty: "x/3A" Reth ve. Inierferometry
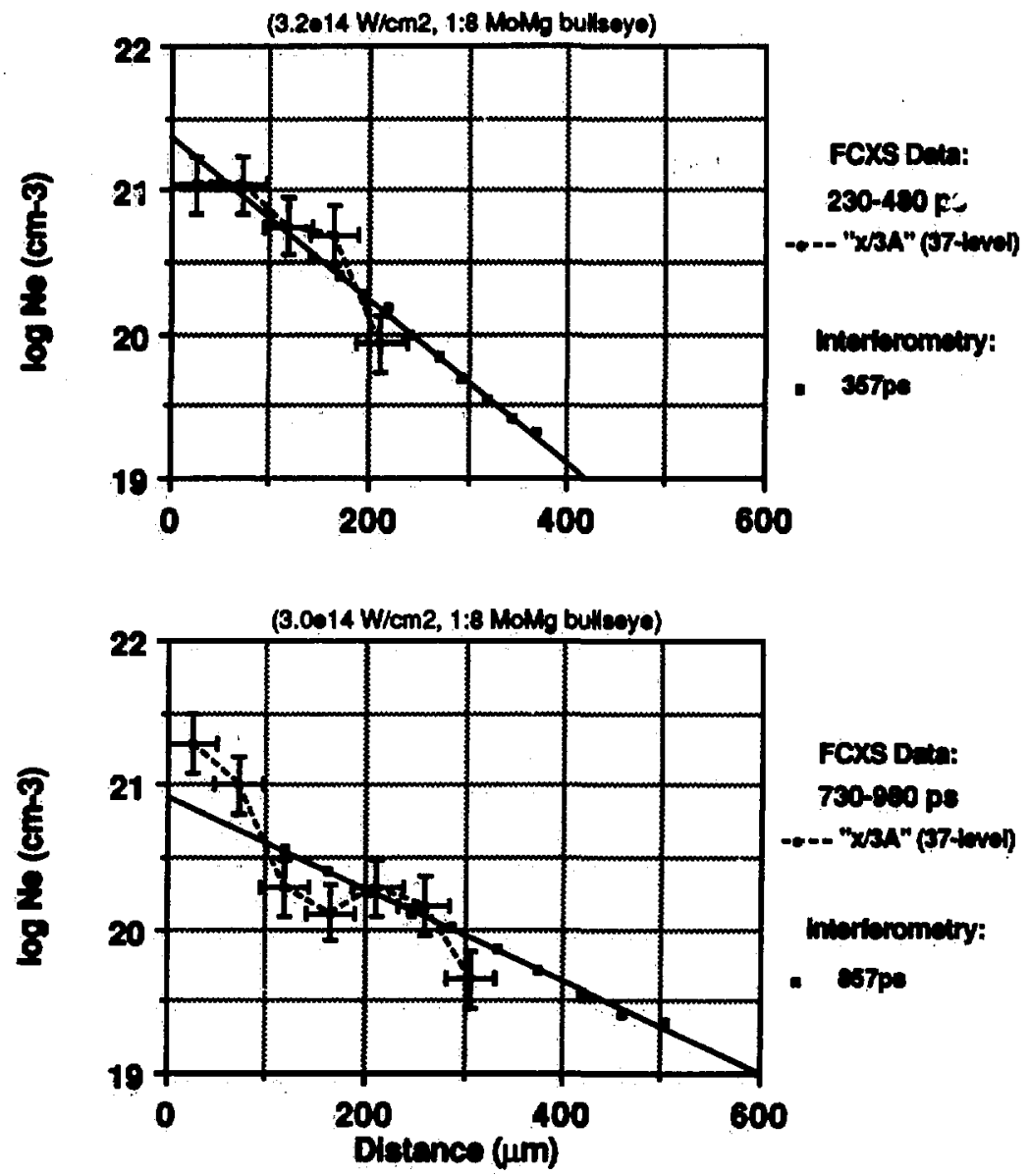

Figure 60 
The 37 level "NELI" model has been used for this analysis. The electron density profile determined from laser interfercmetry on the same target shots and corresponding to approximately the same time intervals as the FCXS data are also plotted (solid points and straight line linear fit). These laser interferometry density values represent the axial density extracted from the Abel inversion.

The " $x / 3 A$ " line ratio density profiles show very good agreement with the density profiles measured with laser interferometry about midway through the FCXS 250 ps time frame (solid points) for the Mo date. Experimental error bars representing the spatial stepsize $( \pm 25 \mu \mathrm{m})$ and estimated $20 \%$ sccuracy in the measured line ratio [approximitely $\Delta\left(\log n_{e}\right) \approx \pm 0.2$ ] are plotted for each data point. The densities determined using the " $x / 3 A$ " line intensity ratio densities fall well within the experimental error bars. The " $x / 3 \mathrm{~A}$ " line ratio densities corresponding to electron temperatures of either $350 \mathrm{eV}$ or $850 \mathrm{eV}$ also lie within the experimental error bars and indicate a minimal sensitivity to variations in the electron temperature for this particular line intensity ratio.

The agreement between experiment and theory is quite good and somewhat surprising since the two models used in this analysis are based entirely on collisional excitation from the Ne-like ground state to feed the excited states in the Ne-like system. As described previously in Chapter 4, the " $\mathrm{x} / 3 \mathrm{~A}$ " line intensity ratio is due to the combined sensitivity of the excited state level populations to variations in the electron density and in particular, the collisional coupling between these and other metastable levels in the Ne-like excited state system. Since both the 37 level "NELI" and more detailed 89 level models both appear to predict the approximately the same " $x / 3 A^{\prime}$ line intensities, contributions from the $(n=4)$ levels do not appear to be significant in this density region. Another possibility is that both the " $x$ " and "3A" levels are coupled to the $n=4$ levels in a similar fashion. 
Another factor is that recombination and ionization processes do not appear to be important for either the " $x$ " or "3A" excited state levels. As shown previously in Figure 37, recombination processes will significantly alter the density dependence of the " $\mathrm{x} / 3 \mathrm{~A}$ " line intensity ratio. Fortunately, for the density and temperature range covered in these measurements $\left(10^{20} \mathrm{~cm}^{-3} \leq \mathrm{n}_{\mathrm{e}} \leq 10^{21} \mathrm{~cm}^{-3}, \mathrm{~T}_{\mathrm{e}}=500 \mathrm{eV}\right.$ ), both the recombination and direct excitation predicted " $\mathrm{X} / \mathrm{A}$ " density dependerces are similar. The $(n=3)-(n=2)$ emission line spectra is dominated by Ne-like (also some F-like and Na-like) lines. If the $(n=3)-(n=2) x$-ray spectra is indicative of the charge state distribution, we can assume that the plasma is mostly Ne-like. It is clear that the plasma is not significantly overstripped to F-like. Therefore, recombinations from F-like $\left[2 s^{2} 2 p^{5}(0,3 / 2) J=3 / 2,2 s^{2} 2 p^{5}(0,1 / 2) J=1 / 2\right.$, and $\left.2 s 2 p^{6}(1 / 2,0) J=1 / 2\right]$ ground states are not expectied to be a major factor in the overall Ne-like excited state population kinetics. The inner-shell ionization rates at temperatures of $500 \mathrm{eV}$ or lower, are relatively amall and ionization of Na-like ions $\left(2 s^{2} 2 p^{6} 3\right)$ are not expected to feed the Ne-like excited states significantly either. Therefore the quasi-steady state analysis, based on the collisional excitation from the Ne-like ground state and detailed $\Delta n=0$ collisional mixing between excited states, appears be sufficiently accurate to sccount for the overall excited state population in the " $x "$ and "3A" levels and in predicting the correct density dependence of the " $\times / 3 A$ " line intensity ratio. 


\subsubsection{The "3G" to " $3 A$ " Resonance Line Ratio}

I have applied a similar line ratio technique to the ratio of two Ne-like resonance lines, "3G/3A", which is related to the line ratio ("3G"+"3F")/("3A"+"3B") used by Bailey et al. (1985) to determine of the electron density. These ratios are also predicted to be density sensitive by the collisional ndiative models. In these new spectra, the intensities for the "3F" and "3B" transitions are affected by blends with other spectroacopic features and therefore, are not included in this analysis. [See Figure 58.] In Figure 61, the experimental " $3 G / 3 A$ " line intensity ratios have been plotted along with the 37 kvel "NELI" model and 89 level model "3G/3A" line ratio predictions as a function of the electron density at $500 \mathrm{eV}$. Both steady state Ne-like collisional radiative Ne-like codes underestimate the " $3 G$ " line intensity (with respect to the " $3 \mathrm{~A}^{n}$ intensity) for densities below $10^{21} \mathrm{~cm}^{-3}$. This is likely due to the sensitivity of the "3G" resonance line to ionization and recombination (in particular, dielectronic recombination) processes. Since the "3G" line corresponds to the lowest energy Ne-like excited level, its level population is enhanced by cascades from higher lying excited levels. This effect has not been included in the collisional radiative models used in this analysis but may contribute significantly to the overall excited state population kinetics. At the low temperatures characteristic to these plasmas, very little F-like ions are generated and are available for recombination back into the Ne-like excited states. Therefore, recombination processes are not expected fully account for the significant differences between the experimentally measured and thearetically predicted "3G/3A" line intensity ratios.

Also, resonance excitation may be an important atomic process which may enhance the excited levels in the $2 s^{2} 2 p^{5} 3$ se-like configuration and has not been included in any detailed L-shell atomic models except for iron. [Goldstein (1988); Smith et al. 
Figure 61. A comparison of experimentally measured and theoretically predicted density dependence of the "3G/3A" line ratio for Ne-like Mo ${ }^{32+}$ (top, Shot \#8502) and $\mathrm{Ag}^{37+}$ (bottom, Shot \#8519). Both the 37 level "NELI" and 89 level Ne-like models have been used assuming $T_{e}=500 \mathrm{eV}$. 

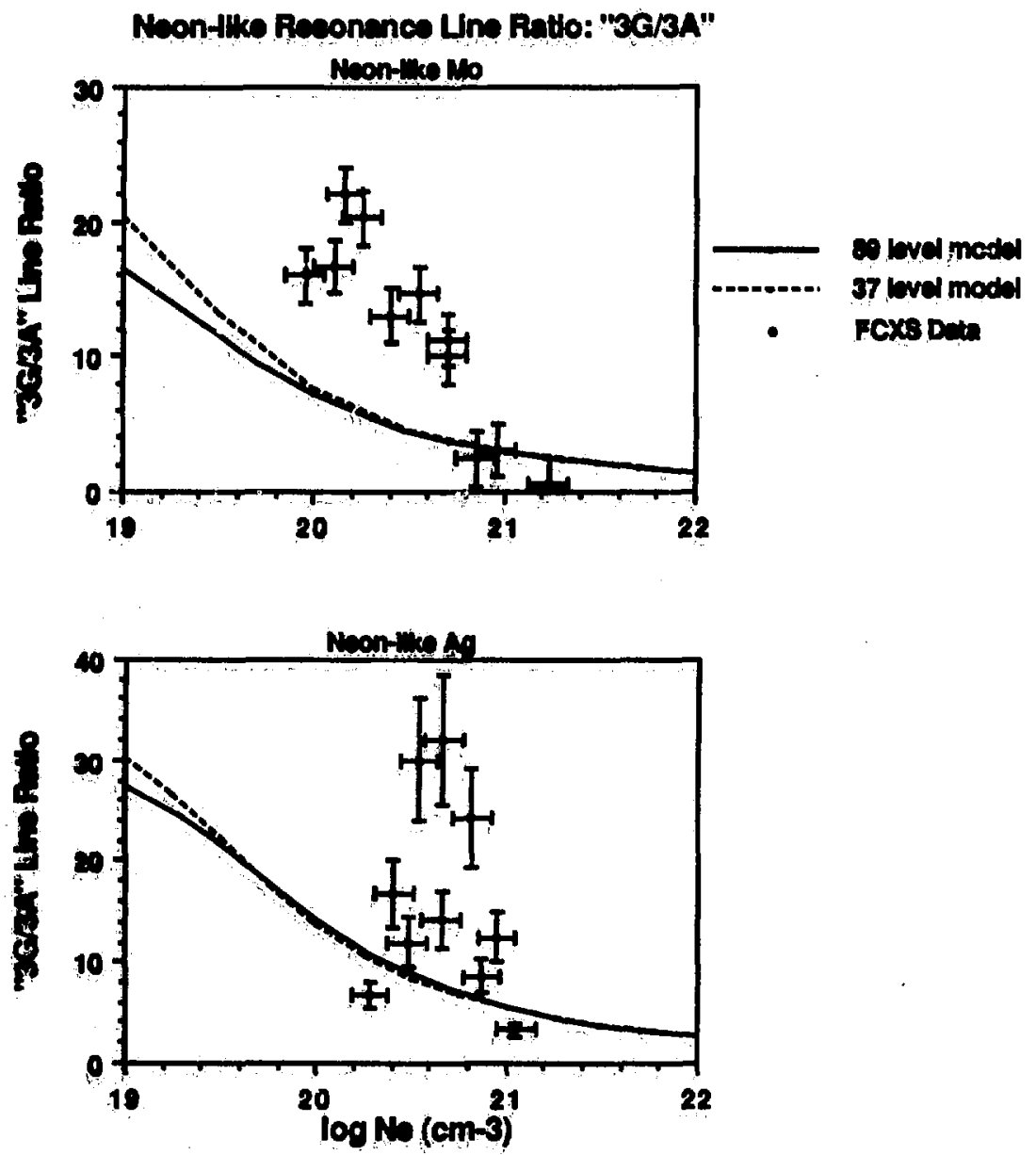

Figure 61 
(1985)] Resonance excitation is closely related to dielectronic recombination. As in dielectronic recombination, the ion first undergoes resonant capture of an electron creating a doubly excited state. The doubly excited state can stabilize by radiative decay of one of the excited electrons (dielectronic recombination). The ion can autoionize by emitting the captured electron, thus relaxing back to its original state. The doubly excited state can also autoionize to a different state (usually a higher-n excited state). Subsequent collisional mixing and radiative decays distribute this additional excited state electron population among the excited levels. The net result is prodicted to be an enhancenent of the $2 s^{2} 2 p^{5} 3$ s configuration populations and therefore the "3F" and "3G" Ne-like resonance line intensities should increase. This problem is currently being addressed and will be included in future Ne-like theoretical models.

\subsubsection{Powdble Short Wavelength Laxing 'Trancitions Between Metastable Leveis in Neon-like Ions}

The measurement of the " $x$ " electric quadrupole line indicates a signinicient population in the $[2 \sin 63 \mathrm{~d}(1 / 2,5 / 2)]=2]$ metastable level. This metastable level zopulation suggests a possible population inversion between this excited level and the $2 s^{2} 2 p^{5} 3 d$ and $2 s^{2} p^{6} 3 p$ level configurations. I refer to these possible short wavelength lasing transitions as "2p-2s" and "3d-3p", respectively. For the "2p-2s" transition, the lower "hsing" levels correspond to the stong dipole allowed "3d-2p" transitions which rapidly decay in an optically thin plasma. These "2p-2s" transitions correspond to $x$-ray transitions with wavelengths of less than $40 \AA$ (approximately $38.6 \AA$ for Mo and $29.3 \AA$ for $A g$ ), below the targeted "water window" goal of current short wavelength laser efforts. [Matthews (1987a; 1987b)] 
I have plotted the predictod gain estimates for various short wavelength $x$-ray transitions in Ne-like $\mathrm{Mo}^{32+}$ and $\mathrm{Ag}^{37+}$ in Figures $62 \mathrm{a}$ and $62 \mathrm{~b}$, respectively, for a range of electron densities between $10^{19}$ and $10^{22} \mathrm{~cm}^{-3}$. These values were obtained frum calculations by A. Osterheld (1988) using the steady state, 89 level Ne-like collisional radintive model. For these calculations, the plasma was assumed to be $100 \%$ Ne-like, and the gains are based on an ion temperature of $250 \mathrm{eV}\left(\mathrm{T}_{e}=\right.$ $500 \mathrm{eV})$. I have plotted the eatimated gains $\left(\mathrm{cm}^{-1}\right)$ for the potential short wavelength "2p-2s" and "3d-3p" transitions as well as the more standard "3p-3s" lasing transitions reported by Natthews et al. (1985; 1987).

Other electric quadripole transitions between the $28^{2} 2 p^{5} 3 p$ level configuration and the $\left.\left[2 s^{2} 2 q^{6}(0,0)\right]-0\right]$ ground save levels may have also been measured although this identificmion can not be certain without a comprehensive analysis of this new data. [See Figures 53 and 55.] Theee "3p-2p" quadrupole lines have been observed in laser probeced pleamins by Geuthier of al. (1986), in low density Tokamak plasmas by Beiridafer et al. (1987) and Rice et al (1988), and in high resolution beam-foil spectroecepy experiments by Dietrich at al. (1985). The density dependence of these quadrupale lines has been modeled by Feldman et al. (1985) for Ne-like ions between silicon and krypon. An extension of this analysis based on laser-produced plasmas seems to be a natural problem to be studied at this point using the new experimentel techniques demonstrnted in the present work. The measurement of the "3p-2p" electric quadrupole transitions can provide a direst measurement (with the appropriate branching ratio) of the upper lasing level population in Ne-like short wavelength laser experiments and may help explain the mysteries surrounding the weak $(\mathrm{J}=0)-(\mathrm{J}=1)$ lasing transitions in the standard Ne-like short wavelength lasing schemes. [Matthews et al. (1985); T.N. Lee et al. (1987)] 
Figure 62. Estimates of gains for short wavelength transitions between metastable levels in Ne-like a) $\mathrm{Mo}^{32+}$ and b) $\mathrm{Ag}^{37+}$ ions assuming $\mathrm{T}_{\mathrm{e}}=\mathrm{T}_{\mathrm{i}}=500 \mathrm{eV}$. The gains shown include the standard "3s-3p" lasing transitions between the singly excited $\mathrm{n}=3$ states (top), and the proposed " $2 \mathrm{~s}-2 \mathrm{p}$ " (middle) and "3p-3d" (bottom) transitions originating from the $\left.\left[2 s 2 \mathrm{p}^{6} 3 \mathrm{~d}(1 / 2,5 / 2)\right]=2\right]$ " $\mathrm{x}^{\text {" and }}\left[2 \mathrm{~s} 2 \mathrm{p}^{6} 3 \mathrm{~d}(1 / 2,3 / 2)\right.$ $\mathrm{J}=2]$ " $x 1$ " excited state levels. The "lasing" transitions are between the level numbers used in the steady state, collisional ndiative 89 level Ne-like model:

\begin{tabular}{|c|c|c|}
\hline \multicolumn{3}{|l|}{ Levels: } \\
\hline $\mathrm{Mo}^{32+}$ & $\Delta q^{37+}$ & Level Confiruration: \\
\hline 3 & 3 & $2 s^{2} 2 p^{5} 3 s(3 / 2,1 / 2) \mathrm{J}=1$ \\
\hline 5 & 5 & $2 s^{2} 2 p^{5} 3 p(3 / 2,1 / 2) J=2$ \\
\hline 8 & 8 & $2 s^{2} 2 p^{5} 3 p(3 / 2,3 / 2) J=2$ \\
\hline 10 & 11 & $2 s^{2} 2 p^{5} 3 s(1 / 2,1 / 2) \mathrm{J}=1$ \\
\hline 13 & 13 & $2 s^{2} 2 p^{5} 3 d(3 / 2,3 / 2) J=1$ \\
\hline 20 & 21 & $2 s^{2} 2 p^{5} 3 p(1 / 2,1 / 2) J=0$ \\
\hline 22 & 19 & $2 s^{2} 2 p^{5} 3 \mathrm{~d}(3 / 2,5 / 2) \mathrm{J}=1$ \\
\hline 23 & 23 & $2 s^{2} 2 p^{5} 3 p(1 / 2,3 / 2) J=2$ \\
\hline 27 & 27 & $2 s^{2} 2 p^{5} 3 d(1 / 2,3 / 2) \mathrm{J}=1$ \\
\hline 31 & 31 & $2 s^{2} p^{6} 3 p(1 / 2,1 / 2) J=1$ \\
\hline 35 & 35 & $2 s 2 p^{6} 3 d(1 / 2,3 / 2) J=2$ \\
\hline 37 & 37 & $2 s^{2} \mathrm{p}^{6} 3 \mathrm{~d}(1 / 2,5 / 2) \mathrm{J}=2$ \\
\hline
\end{tabular}

The wavelengths of the various "lasing" transitions are in parenthesis in angstroms. 


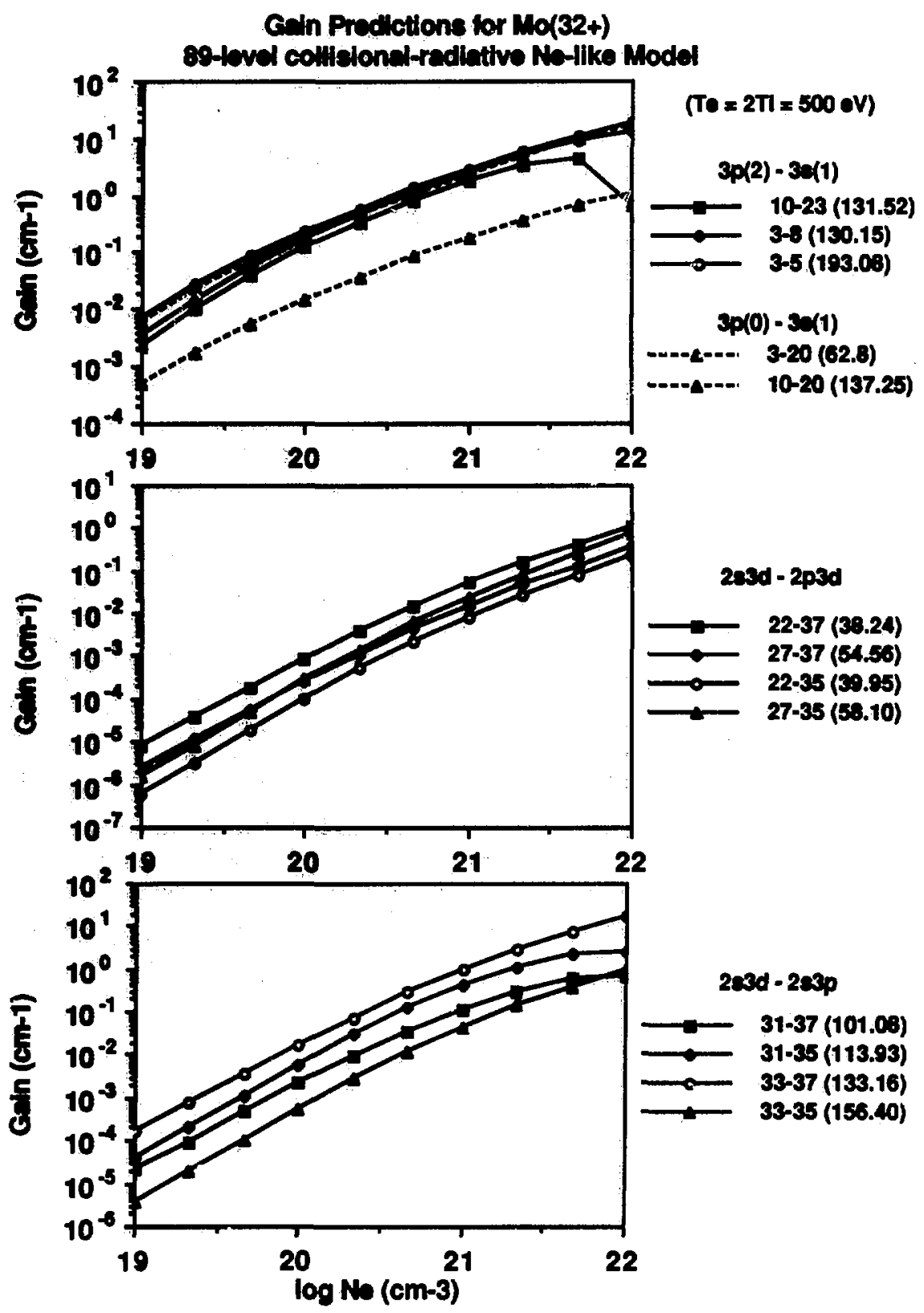

Figure $62 a$ 


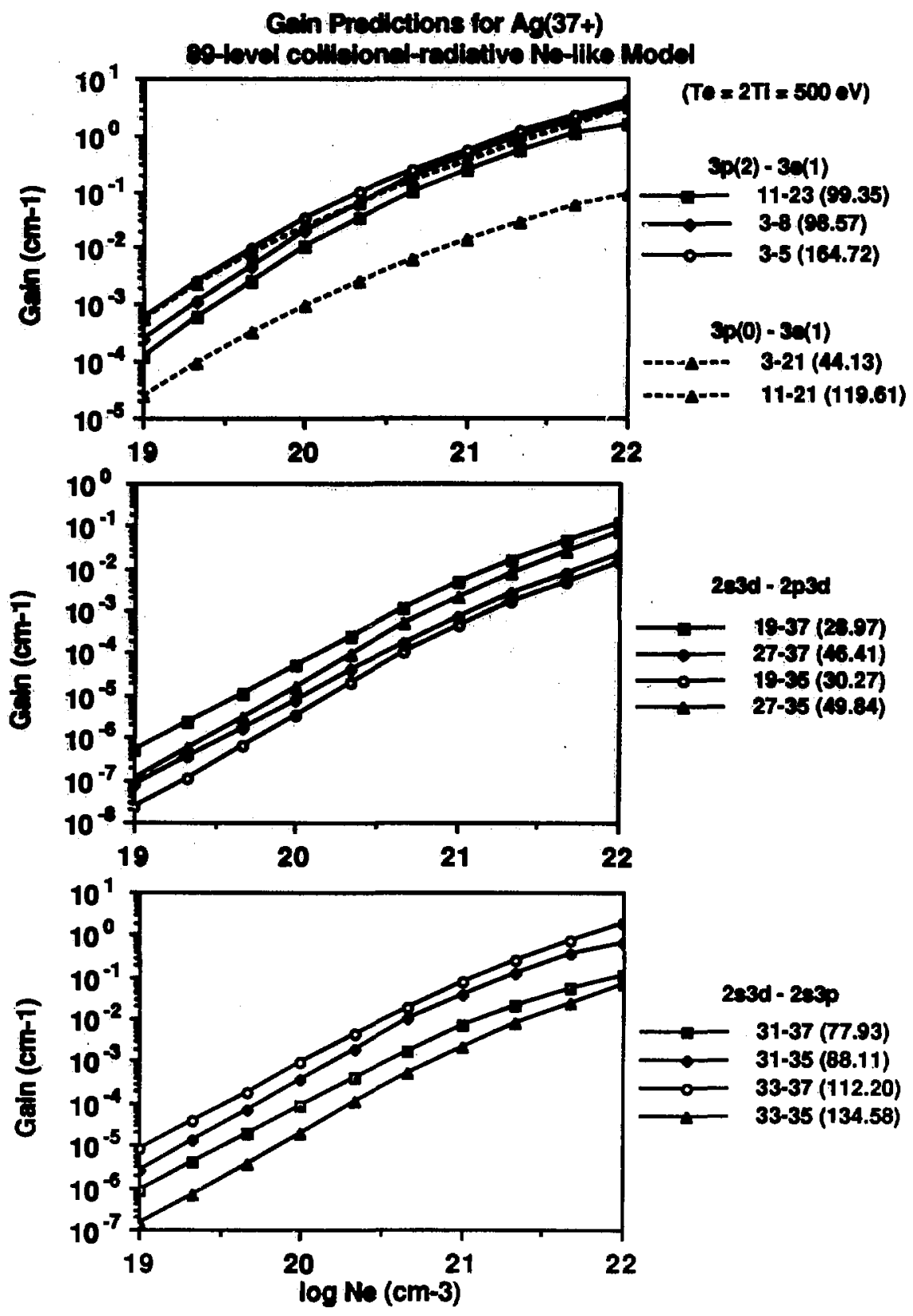

Figure 62b 


\subsection{Summary}

In summary, I have measured simultaneously space and time resolved electric dipole and quadrupole $\mathrm{x}$-ray transitions in Ne-like $\mathrm{Mo}^{32+}$ and $\mathrm{Ag}^{37+}$ ions in high-powered laser-produced plasmas. I have determined the density dependence of these transitions by relating the space and time resolved line intensity profiles with independent measurements of the electron density profiles obtrined simultaneously using holognaphic interfecometry. I have focused on two specific line intensity ratios. The first is the " $\mathrm{x} / 3 \mathrm{~A}$ " eloctric quadrupole to resonance line ratio and the second is the "3G/3A" resonance line ratio. These line intensity ratios are predicted to be sensitive to variations in the electron density based steady state collisional radiative atomic model calculations. The 37 level model "NELI" and a more detailed, steady state, 89 level Ne-like model density dependence predictions for these two Ne-like line intensity ratios wese presented in Chapter 4.

For the " $x / 3 A$ " line ratio, I have found excellent agreement with both steady state Ne-like theoretical predictions. On the other hand, the "3G/3A" resonance line rati does not follow the density dependence predicted by either model and surgests significant shortcomings with either the "quasi-steady state", Ne-like only analysis or perhaps with the atomic processes or rates (such as the resonant excitation) included in the models. One obvious question is why do these quasi-stendy state, collisional ridiative models correctly predict the density dependence of the " $\mathrm{X} / 3 \mathrm{~A}$ " line intensity ratio and not the " $3 \mathrm{G} / 3 \mathrm{~A}$ " resonance line ratio? First, let's consider the general structure of the Ne-like ion. The "x" and "3A" excited states are relatively similar in energy; both levels contain 2s-orbital holes and represent relatively high lying excited levels in the $(n=3)$ Ne-like electron configuration. The " $\mathrm{x}$ " and " $3 \mathrm{~A}$ " excited state levels are directly coupled collisionally (and radiatively) to each other and with many other excited "metastable" levels in the Ne-like system. At high 
densities ( $\mathrm{n}_{\mathrm{e}} \geq 10^{20} \mathrm{~cm}^{-3}$ ), the collisional mixing between the excited Ne-like states is the direct cause of the observed density dependence of the " $x$ " and "3A" line intensity ratio. At $500 \mathrm{eV}$ and $10^{21} \mathrm{~cm}^{-3}$, roughly $30 \%$ of the "x" level population is directly unansferred to the "3A" level via collisions based on the "NELI" calculations listed in Table 5.

We can also examine the experimental conditions. The bulk of the L-shell data presented in this work was measured during the laser heating pulse and basically within the first $250 \mu \mathrm{m}$ of plasma from the target surface. For this region in space and time, the electron densities and temperatures were likely near their peak, "coronal" values $\left(10^{20} \mathrm{~cm}^{-3} \leq n_{e} \leq 10^{21} \mathrm{~cm}^{-3}, T_{e}=500 \mathrm{eV}\right)$. Although $(n=3)-(n=2)$ lines were measured for several charge states including F-like, Ne-like, and Na-like, the spectra was predominantly Ne-like. Therefore, if the $(n=3)-(n=2) x$-ray spectra is indicative of the charge state distribution, one might presume that the plasma is mostly Ne-like and that recombinations and ionization contributions to the overall Ne-like excited state population kinetics will be relatively small. For these temperatures and densities, there may not be a sufficiently large F-like (ground-state) population for recombination processes to be significant. Also, the temperatures are low enough so that inner-shell ionization from Na-like ions is not important either. Therefore, the "quasi-steady state" analysis, based on collisional excitution from the Ne-like ground state, may be sufficiently accurate to account for the overall excited state electron population. The collisional mixing and radiative cascade are properly account for in both steady state Ne-like models. Therefore, both quasi-steady state models correctly predict the density dependence of the "x/3A" line intensity ratio.

On the other hand, the " $3 G$ " and " $3 \mathrm{~A}$ " resonance line excited levels are quite different. Whereas the "3A" level lies fairly high in energy, the "3G" level is a low lying $(n=3)$ excited state level. There is no direct collisional or radiative coupling 
between the "3A" and "3G" excited states. The "3A" and "3G" excited levels are populated differently. As described earlier, the " $3 \mathrm{~A}$ " level is fed mostly via collisional transitions with other high lying "metastable" levels for the density range studied $\left(10^{20} \mathrm{~cm}^{-3} \leq n_{e} \leq 10^{21} \mathrm{~cm}^{-3}\right)$. Because the " $3 G^{\prime}$ level is a low lying excited state, a significant fraction of its population is due to radiative cascades from other Ne-like excited states. This "filtering" radiative cascade path through the "3G" level would be more evident at lower densities where excited state levels depopulate via radiative decay (cascades) rather than by collisional processes. Another factor is that resonant excitation is an important process which may enhance the low lying "3G" excited states and has not been included into the collisional radiative models used for this analysis. This multi-step process should be included and tested to see if the "3G" and related "3F" resonance lines are indeed affected. It is clear that the reltively simple steady state Ne-like models do not correctly model the "3A" and "3G" resonance line intensities. This strongly suggests that a much more detailed, non-stationary theoretical model is required to fully model the physics of $\mathrm{Ne}$-like ions in laser-produced plasmas.

Another important question is why does this relatively simple, collisional radiative model correctly predict the density dependence of the " $\mathrm{x} / 3 \mathrm{~A}$ " line intensity ratio here, and yet similar (electron impact excitation and CRE) $\mathrm{K}$-shell models fail to model the Al "He $/ \mathrm{IC}^{\mathrm{N}}$ data presented in Chapter 5? This might be first explained by differences between the experiments. First, the $\mathrm{Mo}$ and $\mathrm{Ag} \mathrm{L}$-shell resonance lines are optically thin unlike the $\mathrm{Al}$ " $\mathrm{He}_{\alpha}$ " resonance line. As discussed earlier in section 6.3, opacity effects may alter the " $\mathrm{x} / 3 \mathrm{~A}$ " density dependence although not significantly. For the $\mathrm{Al}$ " $\mathrm{He}_{2} / \mathrm{IC}$ " line ratio, the physics of the problem is significantly complicated by plasma opacity effects which distort the overall population kinetics of the ions. 
Secondly, for the plasma region close in near the target surface (between the target surface and $250 \mu \mathrm{m}$ into the plasma), the quasi-steady state collisional radiative models correctly predict the density dependence of the $\mathrm{Mo}^{32+}$ and $\mathrm{Ag}^{37+}$ "x/3A" line ratios. Here, the observed $(n=3)-(n=2) x$-ray emission spectra seems to indicate a predominantly Ne-like plasma. This would also indicate that the plasma is not significantly overstripped and that there is probably only a small F-like charge state fraction available for recombination processes to populate the Ne-like levels. Additionally, the large effect of populations from metastable levels masks the excitation mechanism in these systems. For the Al plasma described in Chapter 5, we found that the plasma was definitely overstripped relative to a CRE electron density and temperature. A significant bare and $\mathrm{H}$-like charge state fraction, combined with the relatively low electron temperatures provided a direct path for populating the He-like excited state levels via recombination processes. Therefore for the $\mathrm{Al}$ plasma, the He-like line intensities ("He, $/ \mathrm{C}$ " and the high-n resonance line ratios) were successfully modeled by the quasi-stendy state recombination model and not by the steady state (CRE) model. Beyond the $250 \mu \mathrm{m}$ region, the " $x$ " and "3A" line intensities are too weak to be measured or conversely, the FCXS's sensitivity set too low. This low density region $\left(\mathrm{n}_{\mathrm{e}} \leq 10^{20} \mathrm{~cm}^{-3}\right)$ should be examined in future experiments to extend this investigation of the density dependence of metastable levels in Ne-like ions. In general, contributions to and from neighboring charge states (recombinations and ionizations) as well as between excited levels (cascades and collisions) must be fully accounted for in a detailed atomic model. 


\section{Chapter 7. Conclusions}

In the present work, I have reported on new simultaneously space and time resolved measurements of the $\mathrm{K}$-shell and L-shell emission spectra which have been used to test the density dependence of several $x$-ray transitions in laser-produced plasmas. The success of these experiments is based on new diagnostic methods to measure the characteristic $x$-ray emission spectrum produced by high-energy, laser-produced plasmas as well as new, detailed, non-stationary atomic models which have been used to interpret the new spectroscopic data. These improved diagnostic methods have significantly reduced spatial and temporal gradients which have compromised the accuracy of previous spectroscopic studies. This work has provided a much clearer, more direct comparison with atomic and plasma theory, thus providing a much better opportunity to truly understand the detailed atomic processes ongoing in these plasmas.

In these experiments, simultaneously space and time resolved Al K-shell x-ray spectra and Mo and Ag L-shell X-ray spectra have been measured for the first time from laser-irradiated microdot targets. Both electron temperatures and densities have been determined independently as functions of both space and time for the Al microdot plasmas. The electron densities were measured using multi-framed holographic interferometry. The electron temperature profiles were determined using the slope of the H-like free-bound recombination continuum when possible. These first ever time resolved measurements of the electron temperature profile and density profiles have been compared to a 2D "LASNEX" hydrodynamic calculation and show significant differences. During the heating laser pulse, the measured electron temperature profile is significantly lower than predicted by the hydrodynamics simulation. At later times, the measured temperature and density profiles are somewhat higher the in the simulation and show evidence of spatial structure that the 
two dimensional calculation does not account for either. This is not well understood and further work is needed to resolve these differences. Experiments of this type with improved spatial and temporal resolution should greatly improve our understanding of the complex laser-matter interaction physics.

The temperature and density dependence of specific spectral line intensities predicted by various theoretical atomic physics models could be clearly tested using the model independent temperature and density measurements. These laser-produced plasmas have been found to be highly transient and standand collisional radiative equilibrium (CRE) model analyses of the spectral data, for the most part, fail. For instance, the density dependence of the Al He-like resonance to intercombination line ratio is correctly predicted using a purely recombining quasi-steady state plasma model of W. Goldstein and A. Osterineld. Both the CRE model and a purely direct excitution populating model underestimate the intensity of the intercombination line. The rocombining quasi-steady state model also successfully models the density sensitive high-n metastable resonance [1 $\left.\operatorname{snp}\left({ }^{1} \mathrm{P}_{1}\right)\right]$ levels in He-like ions. These high-n metastable levels are fed by highly non-stationary 3-body recombination and collisional mixing processes which are not properly socounted for in the present CRE model.

The CRE model seems to work only in limited space-time regions during the evolution of the laser-plasma. For instance, the CRE " $\left.\mathrm{Ly}_{\alpha} /{ }^{1} \mathrm{D}_{2}\right)$ dielectronic satellite" line ratio provides a good measurement of the temperature profile for the plasma near the target surface, and only when the heating laser is on. For all other times, the CRE satellite line ratio fails to correctly predict the electron temperature. For all times and locations in the laser-plasma, the CRE "He $\beta / \mathrm{Ly}_{\beta}$ " resonance line ratio temperatures are much higher that the actual temperatures and indicate that the plasma is overionized with respect to the plasma temperature and density. In both 
cases, recombination processes preferentially feed the H-like and He-like resonance lines and are not accounted for correctly in the steady state CRE model. In future work, the temperature dependence of these line intensity ratios will be tested using the completed, quasi-steady stave K-shell model which will include detailed bare, H-like, He-like, and Li-like level structure and collisional and radiative mixing between the excited state levels.

I have also studied the density dependence of the electric dipole and quadrupole $\mathrm{x}$-rny transitions in neon-like $\mathrm{Mo}^{32+}$ and $\mathrm{As}^{37+}$ in high-density laser-produced plasmas. The density dependence of these transitions have been predicted by two detailed, quasi-ateady state, collisional radiative Ne-like atomic models. I have tested these model predictions by compering theoretical to experimental line intensity ratios and using laser interferometry density profiles and and I have found very good apreement. These atomic models properly account for the collisional mixing between metnetable levels in the excited level structure of Ne-like ions. The precise population kinetics governing the ions in the hot-dense plasmas can be examined by studying the emission line spectra corresponding to $x$-ray transitions from these metastable levels.

There is quite a bit of room for improvements in the design and execution of the experiments presented here. These experiments have just sampled a very limited range of plasma conditions (space, time, laser intensity, laser pulse duration, Z, etc.). For instance, the free-bound recombination continuum temperature measurements should be extended to map out the entire space-time evolution of the microdot target plasma. Ope big improvement in the measurements reported here would be simply using more than one FCXS instrument. This would allow for more spece-time coverage or provide more effective coverage of different spectral regions. The pace and time resolved diagnostics, especially the FCXS, can be improved. Calibration of the FCXS (either absolute or relative) as an integral unit 
would greatly answer any uncertainties in the relative sensitivity of the instruments and the measurements reported here. The space, time, and spectral resolution of the instrument can be increased by a factor of ten or more and would allow for a more precise measurement (smaller plasma gradients) and therefore $a$ better test of atomic theory and plasma models. This would allow for a greater variety of laser-plasma experiments to be conducted. These improvements are based primarily on increasing the overall sensitivity of the MCP detector. The total photon flux into the detector could be increased by going to a focussing (Von Hemos) cryatal configuration or by using a high (integrated) reflectivity multi-layer inetead of a flat Bragg diffraction crystal. A small increase in signal can be achieved by getting the FCXS in clower to the target. Other small but significant improvements can be made by using a fresh CsI photocathode or by using a brighter phosphor (or higher acceleration field). Alternatively, a "coded aperture" design could greatly increase the total flux onto the MCP active surface. The total flux could also be increase by employing a repeller grid in front of the MCP detector. These are just a few suggestions for increasing the overall detector sensitivity used in the FCXS instrument.

This work has demonstrated that simultaneously space and time resolved diagnostics can remove most of the effects of unresolved gradients from $x$-ray spectroscopy data. The great improvement in these spectral measurements oyer past efforts are based on the combined use of small, microdot targets and the development of new simultaneously space, time, and spectrally resolved diagnostics. In the present work, several small but potentially very valuable modifications to the . simple microdot configuration have been used to allow for a tighter control over the experimental plasma conditions. First, composite turgets have been made using a pressed powder technique. This allows for predetermined mixtures of two or more elements to be combined into a single microdot target. This also offers the experimenter another means to control the concentration of potential line emission 
absorbers in the laser-plasma, thus allowing some control over the target opacity. This target fabrication also offers some flexibility in the preparation of the targets. Typically, the exact target design "evolves" as the experiment progresses and therefore, the pressed powder technique allows for the rapid assembly of needed targets. Bull's-eye targets have also been utilized for the first time. These targets also allow for more than one element to be studied at the same time. But the bull's-eye target also provides a small source size (center dot) for high resolution einission line measurements simultaneously with a larger number of ions (in the outer annulus) for weaker spectral features such as the free-bound continuum or stellite lines.

Both types of targets were used without any significant aberrations in either the space-time resolved spectral datu or to the 2D holographic interferometry density pofiles. The target designs require further refinement as slight problems were encountered including the overlapping of spectral features from the different elements in the target. This problem is even more significant for spectroscopic studies since multiple diffraction onders may be present and need to be considered in furure experiments. Also, the elements (target compositior) needs to be better matched hydrodynamicaliy ( $\rho \cdot r)$ to improve the overall one dimensionality of the target. Prelimingry 2D "LASNEX" hydrodynamic simulations show some $( \pm 10 \%)$ transverse temperature and density gradients in the $100 \mu \mathrm{m}$ diameter aluminum microdot plasmas. Both the time integrated pinhole $x$-ray photos and the 2D interferometric density contours do not show significant transverse variations. The free-bound recombination continuum temperatures and the spectral line ratio measurements represent "bulk" or "average" measurements. It is possible that transverse temperature gradients are somehow altering the measured electron temperature profiles but it is difficult to fully account ior the large differences with 
the "LASNEX" hydrodynamic calculations. Further experiments of this type are warranted and they should include the measurement of the full, space resolved (3 dimensional), time resolved temperature profile to actually quantify the transverse (radial) gradients.

Ideally, using smaller targets or by increasing the heating laser focal spot size should help reduce transverse gradients in the plasma. This might also help to understand the low electron temperatures revesled in these experiments and to check for unanticipated gradient effects. Transverse gradients might be measured by using a compcsite microdot target in which diffarent regions of the target are seedicu by different elements. The emission from each element could then be used to determine the temperature distribution across the whole microdot target. This target might resemble a true, multi-ring, bull's-eye or possi" a "slice of fruitcake" where different "flavored" tracer elements are distributed at different radii on a larger base dot. These would be very difficult experiments since each tracer element would have to be studied simultaneously with the sume or better space and time resolution as demonstrated in this work.

A related issue is target opacity and line trapping. I have briefly stated my concerns on the cnude treatment of plasma opacity in current plasma models. At best, the standard escape probability approximation introduces uncertainties into the problem as large as the typical experimental error bars. In general, opacity effects in hot-denee plasmas remain a big unknown. Clearly, dedicated experiments to test the effects of plasme opecity on both line radiation transport and the population rinetics should be done. This would be a natural extension of this present. work using the space and time resolved spectrographs, and the specially designed microdot targets as described earlier. For instance, a systematic study of opacity effects could be conducted by varying the emitter concentration (either be varying the target diameter of the mixture concentration or both) and measuring the corresponding emission 
spectra. Alternatively, some sort of $\mathrm{x}$-ray backlighting experiment would also address the plasm opecity issue.

The denilod characterization of laser-produced plasmas as has been greatly enhanced due to the new, experimentally tochniques and quasi-steady state theoretioal models tested in the present work. This has some at an opportune time with the increaied use of these laser-produced plasmas as mediums for short wavelength lavers and expanded interest in inertial confined fusion research. Althoust there is much more reweurch to be done, this work has been a significant fint wesmonds our increased underetanding of the detuiled atomic and hydrodynamic phytics of these bigh-energy laser-produced plasmas. 


\section{References}

Aglitskii E.V., Antsiferov P.S., Panin A.M., and Ulitin S.A., Opt. Spectrosc. (USSR) 60, 122 (1986).

Alaterre Ph., Popovics C., Geindre J-P., and Gauthier J-C., Opt. Commun. 49, 140 (1984).

Alaterre Ph., Chenais-Popovics C., Audebert P., Geindre J-P., and Gauthier J-C., Phys. Rev. A 32, 324 (1985).

Apruzese J.P., J. Quant. Spectrosc. Rediat. Transfer 33, 71 (1985).

Apruzese J.P., Davis J., Bhatia M., Kepple P.C., and Jacobs V.L., Phys. Rev. Lett. 55, 1877 (1985).

Apruzese J.P., Duston D., and Davis. J., J. Quant. Spectrosc. Radiat. Transfer 36, 339 (1986).

Attwood D.T., Lawrence Livermore National Laboratory Report No. UCRL-77743, 1976 (unpublished).

Attwood D.T., Coleman L.W., and Sweeney D.W, Appl. Phys. Lett. 26, 616 (1975).

Attwood D.T., Sweeney D.W., Auerbach J.M., and Lee P.H.Y., Phys. Rev. Lett. 40, 184 (1978).

Attwood D.T., IEEE Joumal of Quantam Electronics QE-14, 909 (1978).

Aulebert P., Geindre J.P., Gauthier J-C., and Popovics C., Phys. Rev. A. 30, 768 (1984).

Audebert P., Geindre J.P., Gauthier J-C., Alaterre Ph., Popovics C., Cornille M., and Dubau J., Phys. Rev. A. 31,1582 (1984).

Auston D.H., Appl. Phys. Lett. 26, 101 (1975).

Bar-Shalom A., Klapisch M., and Oreg, J., "Electron Collisional Excitation in Complex Spectra of Ionized Heavy Atoms", 1987 (to appear in Phys. Rev. A). 
Bhagevatula V.A. and Yaakobi B., Opt. Commun. 24, 331 (1978).

Bailey J., Stewart R.E., Kilkenny J.D., Walling R.S., Phillips T.W., Fortner R.J., and Lee R.W., J. Phys. B 19, 2639 (1986).

Beirsdorfer P., Bitter M., von Goeler S., Cohen S., Hill K.W., Timbe rlake J., Walling R.S., Chen M.H., Hagelstein P.L., and Scofield J.H., Phys. Rev. A 34, 1297 (1986).

Beirsdorfer P., von Goeler S., Bitter M, Hinnov E., Bell R., Bernabei S., Felt J., Hill K.W., Hulıe R., Stevens J., Suckewer S., Timberlake J., Wouters A., Chen M.H., Scofield J.H., Dietrich D.D., Gerrasimenko M., Silver E., Walling R.S., and Hageletein P.L., Lawrence Livermore National Laboratory Report No. UCRL-97368, 1987 (subnitted to Phys. Rev. A).

Benzel D.M. and Pocha M.D., Rev. Sci. Instrum. 56, 1456 (1985).

Bernard J.E., Baldis H.A., Villeneuve D.M., and Estabrook K., Phys. Fluids 30, 3616 (1987).

Bitur M., Hill K.W., Sauthoff N.R., Efthimion P.C., Meservey E., Roney W., von Goeler S., Horton R., Goldman M., and Stodiek W., Phys. Rev. Lett. 43, 129 (1979).

Boiko V.A., Pikuz S.A., and Fuenow A.Ya, Soy. J. Quant. Electron., 5, 658 (1975).

Boiko V.A., Pikuz S.A., and Faenov A.Ya, Sov. J. Quiant. Eloctron., 5, 394 (1978).

Boiko V.A., Pikuz S.A., and Fienov A.Ya, J. Phys. B 12, 1889 (1979):

Boiko V.A., Chuguanow A.Yu., Frenov A. Ya., Pikuz S.A., Skobelev I.Yu., Vinogradov A.V., and Yukov E.A., J. Phys. B. 12, 213 (1979).

Boiko V.A., Feenov A. Ya., Hahalin S.Ya., Pikuz S.A., Shilov K.A., and Skobelev I.Yu., J. Phys. B. 16, L77 (1983).

Boiko V.A., Skobelev I. Yu., and Faenov A. Ya., Sov. J. Plasma Phys. 10, 82 
(1984).

Booth R., Lawrence Livermore National Laboratory, a private communication (1987).

Burek A., Space Science Instrumentation 2, 53 (1976).

Burkhalter P.G., Nagel D.J., and Cowan R.D., Phys. Rev. A 11, 782 (1975).

Burkhalter P.G., Charatis G., and Rockett P.D., J. Appl. Phys. S4, 6138 (1983).

Burkhalter P.G., Herbst M.J., Duston D., Gardner J., Emery M., Whitlock R.R., Grun J., Apruzese J.P., and Davis J., Phys. Fluids 26, 3650 (1983).

Busch Gar.E., Shepard C.L., Siebert L.D., and Tarvin J.A., Rev. Sci. Instrum. 56,879 (1985).

Cauble R., Lawrence Livermore National Laboratory, a private communication (1987).

Cerjan C., Lawrence Livermore National Laboratory Report No. UCRL-98187, 1988 (submitted to J. Phys. Fluids).

Cerjan C., Lawrence Livermore National Laboratory, a private communication (1988).

Charatis G., KMS Fusion, Inc., a private communication (1:286).

Charatis G., Busch Gar. E., Young B.K.F., and Stewart R.E., J. de Physique (Paris) 49, C1-375 (1987).

Chen M., Phys. Rev. A 31, 1449 (1985).

Chen M., Phys. Rev. A 34, 1037 (1986).

Chen M., Phys. Rev. A 33, 994 (1986).

Chen M., Lawrence Livermore National Laboratory, a private communication (1987).

Christie D.J., Nil.un D.G., Wiedwald J.D., and Wikcox R.B., Rev. Sci. Instrum. 56,812 (1985).

Christie D.J., Lawrence Livermoie National Laboratory, a private communication - 
"LEA84-2685, (1984).

Cowan, R.D., The Theory of Atomic Smucture and Spectra, (University of

California Press, Berkeley, 1981).

Criss J.W., Applied Spectroscopy 33, 19 (1979).

Davis J., Whitney K.G., and Apruzese J.P., J. Quant. Spectrosc. Radiat. Transfer 20, 353 (1978).

DeMichelis C. and Mattioli M., Nucl. Fusion 21, 677 (1981).

Dietrich D.D., Chandler G.A., Forther R.J., Hailey C.J., and Stewart R.E., Phys. Rev. Lett. S4, 1008 (1985).

Donaldeon T.P., Hutcheon R.J., and Key M.H., J. Phys. B 6, 1525 (1973).

Donaldeon T.P., Hutcheon R.J., Key M.H., and Umquhart R.J., J. Phys. B 9, 1645 (1976).

Drake G.W.F. and Dalgarno A., Astrophys. J. 157, 459 (1967).

Drake G.W.F., Astrophys. J. 158, 1199 (1969).

Drake G.W.F., Victor G.A., and Dalgarno A., Phys Rev. 189, 25 (1969).

Drake P., Young P.E., Williams E.A., Eetabrook K., Kruer W.L., Lasinski B.F., Darrow C.B., Baldis H.A., and Johnson T.W., Phys. Fluids 31, 1795 (1988).

Dubau J. and Volonte S., Rep. Prog. Phys. 43, 199 (1980).

Dubau J., Gabriel A.H., Loulergue M., and Siceman-Clark L., and Volonte S., Mon. Not. R. Astr. Soc. 195, 705 (1981).

Duston D., Davis J., and Kepple P.C., Phys. Rev. A 24, 1505 (1981).

Duston D., Rogerson J.E., Davis J., and Blaha M., Phys. Rev. A 28, 2968 (1983).

Duston D., Procestinge of the 2nd Inremntional Conference on Redintive Properties of Hot Dene.Mntr, edited by J. Davis et al., page 107 (World Scientific Singapore 1985).

Dworaky L.N., Modem Trunsmission Line Theory and Applications, 66, (John Wiley \& Sons., New York, 1979). 
Eckart M.J., Lawrence Livermore National Laboratory, a private communication (1985).

Elton R.C., Lee T.N., and Molander W.A., J. Opt. Soc. Am. B 4, 539 (1987).

Estabrook K. and Kruer W.L., Phys. Rev, Lett. 40, 42 (1978).

Fabbro R., Max C., and Fabre E., Phys. Fluids 28, 1463 (1985).

Fehl D.J., Cheng J., Kuswa, G.W., and Mendel C.W., Rev. Sci. Instrum. 51, 292 (1980).

Feldman U. and Doechek G.A., J. Opt. Soc. Am. 67, 726 (1977).

Feldmen U., Bhatia A.K., and Suckewer S., J. Appl. Phys. S4, 2188 (1983).

Feldman U., Seeley J.F., and Bhatia A.K., J. Appl. Phys. S6, 2475 (1984).

Feldman U., Seeley J.F., and Bhatia A.K., J. Appl. Phys. 58, 3954 (1985).

Finn H.F. and Hummell M., Lawrence Livurmore National Laboratory Report No. UCID-17100, 1976 (unpublished).

Fraser G.W., Berstow M.A., Peurson J.F., Whiteley M.J., and Lewis M., Nucl.

Instr. and Meth. 224, 272 (1984).

Fujimoto T. and Kato T., Phys. Rev. A 30, 379 (1984).

Gabriel A.H. and Paget T.M., J.Phys, B. 5, 673 (1972).

Gabriel A.H., Mon. Not. R. Astr. Soc. 169,99 (1972).

Gabriel A.H. and Joadan C., Crestudier in Aromic Collision Physice II, edited by

MaDeniel E.W. und McDowell M.R.C., page 209 (Ameriçan Elsevier, NY 1972).

Gauthier J-C., Geindre J-P, Najmabadi K., Popovic C., Poquerusse A. and

Weinfeld M., J. Phys D. 16, 1929 (1983).

Gauthier J-C., Geindre J-P., Monier P., Chenais-Popovics C., Wyart J-F., and

Luc-Koenit E., Proc 3nt Conf. on Rediative Properties of Hot and Dense

Mnter. Williamebure, Va. 154 (1985).

Gauthier J-C., Geindre J-P., Monier P., Luc-Koenig E., and Wyart J-F., J. Phys.

B 19, L385 (1986). 
Gauthier J-C., Proceedings of the SPIE 831, 1 (1987).

Glinsky M., Lawrence Livermore National Laboratory, a private communication Internal Memo "RP-85-105 on "ROCKIT" crystal reflectivity rocking curve calculating code, 1985 (unpublished).

Goldstein W.H., Walling R.S., Bailey J., Chen M.H., Fortner R., Klapisch M., Phillips T., and Stewart R.E., Phys. Rev. Lett. 58, 2300 (1987).

Goldstein W.H., Walling R.S., Phys. Rev. A 36, 3482 (1987).

Goldstein W.H., Whitten B.L., Haxi A.U., and Chen M.H., Phys. Rev. A 36, 3607 (1987).

Goldstein W.H., Oreg J., Zigler A., Bar-Shulom A., and Klapisch M., Lewrence Livermore National Laboratory Report No. UCRL-97858, 1988 (submitted to Phys. Rev. A).

Goldstein W.H., Lawrence Livermore National Laboratory, a private communication (1988).

Gordon H., Hobby M.G., and Peacock N.J., J. Phys. B. 13, 1985 (1980).

Grant I.P., J. Phys. B 7, 1458 (1974).

Grant I.P., McKenzie B.J., Norrington P.H., Mayers D.F., and Pyper N.C., Comput. Phys. Comm. 21, 207 (1980).

Grunt I.P. and McKenzie B.J., J. Phys. B. 13, 2671 (1980).

Griem H., Birma Spectroacopy, (McGraw-Hill, New York, 1964).

Griem H., Phys. Rev. A 33, 3580 (1986).

Grekhov I.Y., Kurdo-Sywoev A.F., Kostina L.S., and șhenderey S.V., Electron. Lett. 17, 422 (1981).

Hagelstein P.L., Ph. D. Thesis, Massachusetts Institute of Technology, 1981 (unpublished).

Hagelewein P.L. and Jung R.K., ADNDT 37, 121 (1987).

Hagelstein P.L., Rosen M.D., and Jacobs V.L., Phys. Rev. A 34, 1931 (1986). 
Hazi A.U., Lawrence Livermore National Laboratory, a private communication (1987).

Helava H., Ejchenberger C., Gilman C., and Schneider R., Bull. of the Am Phys. Soc. 24 (1971).

Henke B.L., Knangr J.P., and Premaratne K., J. Appl. Phys. 52, 1509 (1981).

Henke B.L., Lee P., Tanaka T.J., Shimabukuro R.L., and Fujkawa B.K., Atomic Datr and Nralenr Dan Trbles 27, (Academic, New York, 1982).

Henke B.L., Uejio J.Y., Stone G.F., Dittmore C.H., and Fujiwara F.G., J. Opt. Soc. Am. B 3, 1540 (1986).

Herbat M.J., and Gun J., Phys. Fluids 24, 1917 (1981).

Herbat M.J., Burthalier P.G., Grun J., Whitlock R.R., and Fink M., Rev. Sci. Instrum. 53, 1418 (1982).

Ito M., Kume H., and Oba K., IEEE Trans. Nucl. Sci. NS-31, 408 (1984).

Jaegle P., Jamelot G., Carillon A., Klisnick A., J. de Physique (Paris) 47, C6-31 (1986).

Jaegle P., Carillon A., Klisnick A., Jamelot G., Guennou H., and Sureau A., Europhys. Lett. 1, 555 (1986).

Jeeqle P., Jamelot G., Carillon A., Klisnick A., Surean A., and Guennou H., J. Opt. Soc. Am. B 4, 563 (1987).

KMS Furion, Inc. Annual Technical Report, 1982 (unpublished).

Kaplan S.A. and Pikelner S.B., The Interentelir Medium (Harvard Univeraity Press, Cambridge, Mass, 1970).

Karzas W.J. and Latter R., Astrophys. J. Suppl. Ser. 6, 167 (1961).

Kunfiman R.L., Lee R.W., Matthews D.L., and Kilkenny J.D., J. Quant. Spectroce. Radiat. Tranefer 32, 335 (1984).

Kanftiman, R.L., Eetabrook K.G., and Lee R.W., Broc. 3rd.Conf, on Radintive Pronerties of Hor and Dence Mntrex, Williamsburg, Va 128 (1985). 
Kauffman R.L., Lee R.W., and Estabrook K., Phys. Rev. A. 35, 4286 (1987).

Key M.H., et al., Rutherford Laboratory Report. RL 77 122/B, 1977 (unpublished).

Key M.H. and Hutcheon R.J., Adv. in Atomic and Molec. Physics 16, 201 (1980).

Key M.H., Lewis C.L.S., Lunney J.G., Moore A., Ward J.M., and Thareja R.K., Phys. Rev. Lett. 44, 1669 (1980).

Key M., et al., J. de Physique (Paris) 47, C6-71 (1986).

Kilkenny J.D., Lee R.W., Key M.H., and Lunney J.G., Phys. Rev. A 22, 2746 (1980),

Kilkenny J.D., Bell P., Power G., Hunks R., Turner R.E., and Wiedwald J., "Comparieon of Measurements and Modeling of Sub $100 \mathrm{psec}$ Photoelectron Gating with Microchannel Plates", presented at the 1988 National Laboratories Photonics Worishop, Berkeley, CA, June 1-2, 1988, (unpublished).

Klapisch M., Computer Phys. Comm. 2, 239 (1971).

Klapisch M., Schwab J.L., Fraenkel B.S., and Oreg J., J. Opt. Soc. Am. 61, 148 (1977).

Koo J.C., McWright G.M., Pocha M.D., and Wilcox R.B., Appl. Phys. Lett. 45, 10 (1984).

Koppel L.N. and Eckels J.D., Lawrence Livermore National Laboratory Report No. UCRL-79781, 1977 (unpublished).

Kunze H.J., Gabriel A.H., and Griem Hans R., Phys. Rev. 165, 267 (1968).

Lamoureux M., Moller C., and Jaegle P., Phys. Rev. A 30, 429 (1984).

Lamoureux M., Moller C., and Jaegle P., J. Quant. Spectrosc. Radiat. Transfer 33, 127 (1985).

Lamoureux M., a private communication (1986).

Lengdon A.B., Phys. Rev. Lett. 44, 575 (1980).

Lee R.W., J. Quant. Spectrosc. Radiat. Transfer 27, 87 (1982). 
Lee R.W., Matthews D.L., Lane S.M., Campbell E.M., Koppel L., Scofield J., Auerbach J., and Lim T., J. Phys. B 15, L317 (1982).

Lee R.W., and Salter J.M., Broceedings of the 2nd International Conference on Badintive Properties of Hgt Dense Matter, odited by J. Davis et al., page 37 (World Scientific Singspare 1985).

Lee R.W., Matthews D.L., Koppel L., Busch G.E., Charatis G., Dunning M.J., and Mayer F.J., J. Appl. Phys. 54, 4090 (1983).

Lee R.W., Kilkenny J.D., Kauffman R.L., and Matthews D.L., J. Quant. Spuitrosc. Radiat. Transfer 31, 83 (1984).

Lee R.W., Whitten B.L., and Strout R.E., J. Quant. Spectrosc. Radiat. Transfer 32,91 (1985).

Lee R.W., J. Appl. Phys. 58, 612 (1985).

Lee R.W. and Estabrook K.G., Phys. Rev. A 35, 1269 (1987).

Lee T.N., McLean E.A., and Elton R.C., Phys. Rev. Lett. 59, 1185 (1987).

London R., Lawrence Livermore National Leboratory, a private communication (1986).

Lunney J.G., Ph.D. Thesis, Queen's University, Belfast, Northern Ireland, 1979 (unpublished).

MacGowan B.J., Rosen M.D., Eckart M.J., Hagelwein P.L., Matthews D.L., Nileon D.G., Phillipe T.W., Scofield J.H., Shimkaveg G., Trebes J.E., Walling R.S., Whitten B.L., and Woodworth J.G., J. Appl. Phys. 61, 5243 (1987).

Majoribanks R.S., Richardeon M.C., Audebert P.R., Bradley D.K., Gregory G.G., and Jaunimagi P.A., Proceedings of the SPIE 831, 185 (1987).

Marrs R.E., Dietrich D.D., Fortner R.J., Levine M.A., Price D.F., R.E. Stewart, and Young B.K.F., Appl. Phys. Lett. 42, 946 (1983).

Matthews D.L., Camphell E.M., Ceglio N.M., Hermes G., Kauffman R., Koppel L, Lee R., Manes K., Rupert V., Slivinsky V.W., Tumer R., Ze F., J. Appl. 
Phys. 54, 4260 (1983).

Mathews D.L., Kauffman R.L., Kilkenny J.D., and Lee R.W., Appl. Phys. Lett. 44, 586 (1984).

Matthews D.L., Hagelstein P.L., Rosen M.D., Eckart M.J., Ceglio N.M., Hazi A.U., Medecki H., MacGowan B.J., Trebes J.E., Whitten B.L., Campbell E.M., Hatcher C.W., Hawryluk A.M., Kauffman R.L., Pleasance L.D., Rambach G., Scofield J.H., Stone G., and Weaver T.A., Phys. Rev. Lett. S4, 110 (1985).

Matthews D.L., Lawrence Livermore National Laboratory Report No. UCRL-96579, 1987 (unpublished).

Matthews D.L., Rosen M., Brown S., Ceglio N., Eder D., Hawryluk A., Keane C. London R., MacGowan B., Maxon S., Nileon D., Scofiled J., and Trebes J., J. Opt. Soc. Am. B 4, 575 (1987).

Max C.E., Proceedingr of the Les Houches Summer School of Theoretical Physics, edived by R. Balien and J.C. Adem (North-Hollend Amsterdam, 1981) 34, 301. McWhirter R.W.P., Phrmn Dinmontic Techninine, odited by R.H. Huddlestone and S.L. Leonard, page 201 (Academic Press, NY-London 1965).

Mihalas D., Strellnr Atmoneheres, (W.H. Freeman \& Co., San Francisco 1978). NRL. Pinemn Formilng, D.L. Book ed., The Office of Naval Research, 1990 (unpublishod).

Nagel D.J., Burbhulter P.G., Dozier C.M., Holrichter J.F., Klein B.M., McMahon J.M., Stamper J.A., and Whitlock R.R., Phys. Rev. Lett. 33, 743 (1974).

Nahrath B., Blanz W.E., and Decker G., Rev. Sci Instrum. 48, 95 (1977). Nuckolls J., Wood L, Thiessen A., and Zimmerman G., Nature (London) 239, (1972).

Oba K., Humamateu TV Co., Intemal Report * RES-0792-01, (unpublished). ONeill F., Davis G.D., Gower M.C., Turcu L.C.E., Lawless M., and Williams M., Proceedings of the SPIE 831, 230 (1987). 
Osterbrock D.E., Astrophysics of Gaseous Nebulae, (W.H. Freeman \& Co., San Francisco 1974).

Osterheld A.L., Lawrence Livermore National Laboratory, a private communication (1988).

Pierce E.L., Applied Optics 19, 952 (1980).

Premaratne K., Dietz E.R., and Henke B.L., Nucl. Instr. and Meth. 207, 465 (1983).

Presnyakov L.P., Sov. Phys. Usp. 19, 387 (1977).

Price D.F., Dietrich D.D., Fortner R.J., Lee Y.T., Marrs R.E., Stewart R.E., and Young B.K.F., Bull. Am. Phys. Soc. $5 U 13$ (1983).

Price D.F., Ph.D. Thesis, University of California, 1988 (unpublished).

Reed K., Lawrence Livermore National Laboratory, a private communication (1987).

Rice J.E., Marmar E.S., Terry J.L, Daley K.F., Whitten B.L., Walling R.S., Scofield J.H., and Chen M.H., "X-ray Obeervations of Krypton in the Neon-Like and Adjacent Ionization Stages from the ALCATOR C Tokamak", (to be published 1988).

Rockett P.D., Bird C.R., Hailey C.J., Sullivan D., Brown D.B., and Burkhnlter P.G., Appliod Optics 24, 2536 (1985).

Rockett P.D., Bird C.R., Hilley C.J., Koenig Z., Lupton J., and Goddes J., Proceedings of the SPIE 69, 114 (1986).

Rosen M.D., Hagelstein P.L., Mathews D.L., Campbell E.M., Hazi A.U., Whitten B.L., MacGowan B.J., Tumer R.E., Lee R.W., Charatis G., Busch Gar.E., Shepard C.L., and Rockett P.D., Phys. Rev. Lett. 54, 106 (1985).

Rosen M.D., Trebes J.E., MacGowan B.J., Hagelstein P.L., London R.A., Mathews D.L., Nilson D.G., Phillips T.W., Whelan D.A., Charatis G., Busch G.E., Shepard C.L., and Jacobs V.L., Phys Rev. Lett. 59, 2283 (1987). 
Rosen M.D., Lawrence Livermore National Laboratory, a private communication (1987).

Rybicki G.B. and Lightman A.P., Radiative Processes In Astrophysics, (John Wiley \& Sons, New York 1979).

Saloman E.B., Pearlman J.S., and Henke B.L., Appl. Opt. 19, 749 (1980).

Samson J.A.R., Nucl. Instr. and Meths. 222, 215 (1984).

Scofield J., Lawrence Livermore National Laboratory, a private communication (1987).

Seely J.F., Phys. Rev. Lett. 42, 1606 (1979).

Seely J.F., Phillips T.W., Walling M.D., Bailey J., Stewart R.E., and Scofield J.H., Phys. Rev. A 34, 2942 (1986).

Skobelev I.Yu., Vinagradov A.V., and Yukov E.A., Physica Scripta 18, 78 (1978).

Smith B.W., Raymond J.C., Wann J.B., and Cowan R.D., The Astrophysical Journal 293, 898 (1985).

Spizzer L., Bhysics of Bully Innired Gnes, (Wiley -Interecience, NY-London 1962).

Stavrakas T.A. and Lee R.W., J. Phys. B. 15, 1939 (1982).

Steinman D.A., et al., Am. Vacuum Soc. PS-MoA9, 1987 (unpublished).

Stewart R.E., Ph. D. thesis, University of Californit, 1983 (unpublished).

Stone S.R., and Weisheit J.C., J. Quant. Spectrosc. Radiat. Transfer 35, 67 (1986).

Suckewer S., Skinner C.H., Milchberg H., Keane C., and Vooxhees D., Phys. Rev. Lett. 55, 1753 (1985).

Suckewer S., Skinner C.H., Kim D., Valeo E., Voorhees D., and Wouters A., .' de Physique (Paris) 47, C6-23 (1986).

Suckewer S., Skinner C.H., Kim D., Valeo E., Voorhees D., and Wouters A., 
Phys. Rev. Lett. 57, 1004 (1986).

Sweeney D.W., J. Opt. Soc. Am. 64, 559 (1974).

Tomic T., Koyama K., Atoda N., Komeiji S., Matsumoto Y. Yaoita A., Matsushima I., and Yano M., Proceedings of the SPIE 831, 224 (1987). Trebes J., Brown S., Campbell E.M., Ceglio N., Eder D., Gaines D., Hawryluk A., Keane D., London R., MacGowan B., Matthew D., Maxon S., Nilson D., Rosen M., Stearns D., Stone G., and Whelan D., Proceedings of the SPIE 831, 258 (1987).

Vainstein L.A., Soviet Phys.-JETP 40, 32 (1975).

Van Regemorter H., Astrophys. J. 136, 906 (1962).

Varian Associates, Inc., Image Tube Division, Type VUW-8920 (uncoated, $\mathrm{H}_{2}$-fired only).

Vinogradov A.V., Skobelev I.Yu., and Yukov E.A., Sov. J. Quant. Electron., 5, 630 (197)

Walling R.S., Ph.D. thesis, University of California, 1988 (unpublished).

Walling R.S., Lawrence Livermore National Laboratory, a private communication (1988).

Weisheit J.C., J. Phys. B. 8, 2556 (1975).

W'eisheit J.C., Tarter C.B., Scofield J.H., and Richards L.M., J. Quant., Spectrosc. Radiat. Tranfer 16, 659 (1976).

Weisheit J.C., Lawrence Livermore National Laboratory Report No. UCRL-89653, 1983 (unpublished).

Weisheit J.C., Lawrence Livermore National Laboratory Report No. UCID-20744, 1986 (unpublished).

Whiteley M.J., Peareon J.F., Fraser G.W., and Barstow M.A., Nucl. Instr. and Meths. 224, 287 (1984). 
Whitten B.L., Hazi A.U., Chen M.H., and Hagelstein P.L., Phys. Rev. A 33, 2171 (1986).

Whitten B.L., Hazi A.U., Keane C.J., London R.A., MacGowan B.J., Phillips T.W., Rowen M.D., and Whelen D.A., Lawrence Livermore National Laboratory Report No. UCD-21152, 1987 (unpublished).

Willi O., Lawrence Livermore National Laboratory Report No. UCRL-15930, 1987 (unpublished).

Wikox R.B., Lawrence Livermore National Laboratory Report No. UCRL-91881, 1985 (unpublished).

Wilson R., J. Quant. Spectrosc. Radiat. Transfer 2, 477 (1962).

Wiza J.L., Nucl. Instr. and Meths. 162, 587 (1979).

Yaakobi B. and Nee A., Phys. Rev. Lett 31, 350 (1977).

Yaukobi B., Burke P., McCrory R., Seka W., Soures J.M., Burke A.J., and Deslaties R.E., Opt. Commun. 38, 196 (1981).

Yamazald I., Trmai N., Kume H., Tsuchiya H., and Oba K., Rev. Sci. Instrum. S6, 1187 (1985).

Young B.K.F., Stewart R.E., Bailey J., and Woodworth J.G., Rev. Sci. Instrm. 57,2729 (1986).

Youns B.K.F., Charatis G., Busch Gar.E., Roven M.D., and Stewart R.E., Proceedings of the SPIE 831, 18 (1987).

Young B.K.F., Charatis G., Busch Gar.E., Cerjan C.J., Goldstein W.H., Onecheld A.L., Phillips T.W., Roven M.D., Stewart R.E., and Walling R.S., Procoedings of the SPIE 913, (to be published).

Young B.K.F., Stewert R.E., Cerjan C.J., Charatis G., and Busch Gar.E., Lawrence Livermore National Laboratory Report No. UCRL-98692, 1988 (submitued to Phys. Rev. Letts).

Young B.K.F., Omerheld A.L., Walling R.S., Goldstein W.H., Phillips T.W., 
Stewart R.E., Charatis G., and Busch Gar.E., "Measurement of Density Sensitive Electric Quadrupole Transitions in Neon-like Laser-Produced Plasmas", (submitted to Phys. Rev, Letts).

Zigler A., Kolbe J., and Lee R.W., Appl. Phys. Lett. 50, 1133 (1987).

Zimmerman G.B. and Kruer W.L., Commun. Plasma Phys. Controlled Fusion 2, 85 (1975). 